UNIVERSIDADE DE SÃO PAULO

FFCLRP - DEPARTAMENTO DE BIOLOGIA

PROGRAMA DE PÓS-GRADUAÇÃO EM ENTOMOLOGIA

\title{
Phylogenetic relationships of corbiculate bees (Hymenoptera: Apidae: Apini)
}

\author{
Relações filogenéticas entre abelhas corbiculadas \\ (Hymenoptera: Apidae: Apini) \\ DIEGO SASSO PORTO
}

Dissertação apresentada à Faculdade de Filosofia, Ciências e Letras de Ribeirão Preto da USP, como parte das exigências para a obtenção do título de Mestre em Ciências, Área: Entomologia 
UNIVERSIDADE DE SÃO PAULO

FFCLRP - DEPARTAMENTO DE BIOLOGIA

PROGRAMA DE PÓS-GRADUAÇÃO EM ENTOMOLOGIA

\title{
Phylogenetic relationships of corbiculate bees (Hymenoptera: Apidae: Apini)
}

\author{
Relações filogenéticas entre abelhas corbiculadas \\ (Hymenoptera: Apidae: Apini) \\ DIEGO SASSO PORTO
}

Orientador: Eduardo Andrade Botelho de Almeida

Dissertação apresentada à Faculdade de Filosofia, Ciências e Letras de Ribeirão Preto da USP, como parte das exigências para a obtenção do título de Mestre em Ciências, Área: Entomologia 
Autorizo a reprodução e divulgação total ou parcial deste trabalho, por qualquer meio convencional ou eletrônico, para fins de estudo e pesquisa, desde que citada a fonte.

\section{FICHA CATALOGRÁFICA}

Porto, Diego Sasso

Phylogenetic relationships of corbiculate bees (Hymenoptera: Apidae: Apini). Ribeirão Preto, 2015.

$223 \mathrm{p}$.

Dissertação de Mestrado, apresentada à Faculdade Filosofia, Ciências e Letras de Ribeirão Preto/USP. Área de concentração: Entomologia.

Orientador: Almeida, Eduardo Andrade Botelho de.

1. Systematics. 2. Comparative Morphology. 3. Eusociality. 4. Apina. 5. Meliponina. 
Dedico aos meus pais e amigos que sempre me apoiaram nos momentos de dificuldades e a todos os meus mestres que sempre me guiaram pelos caminhos do conhecimento, sem os quais esta dissertação jamais teria sido produzida. 


\section{Agradecimentos}

Ao Programa de Pós-Graduação em Entomologia e ao Departamento de Biologia da FFLCRP por propiciarem as condições necessárias para que esse trabalho fosse executado. Ao Natural History Museum of Denmark, University of Copenhagen por me receber e oferecer toda a infra-estrutura necessária para a execução do estágio de pesquisa no exterior.

À FAPESP pela bolsa de mestrado (proc. \#2012/22261-8) e bolsa de estágio de pesquisa no exterior (BEPE, proc. \#2014/10090-0), ambos vinculados ao projeto de jovem pesquisador concedido ao Dr. Eduardo A. B. Almeida (proc. \#2011/09477-9).

Ao orientador e amigo Dr. Eduardo A. B. Almeida pelos valiosos ensinamentos, tempo e dedicação ao longo da orientação deste projeto de mestrado. Muito obrigado por ter me introduzido ao admirável universo das abelhas e por ter me acolhido em seu laboratório quando mais precisei.

A todos os docentes do Programa de Pós-Graduação em Entomologia pelos ensinamentos, conselhos e proveitosas discussões ao longo das disciplinas do mestrado.

À Maria Isabel P. Balbi pelo auxílio dado ao longo dos primeiros clareamentos e dissecções de espécimes de abelhas.

Ao Dr. Dalton Amorin pelos ensinamentos e orientação ao longo da minha iniciação científica. Obrigado por me introduzir ao estudo dos insetos, da morfologia comparativa e da sistemática filogenética.

Ao técnico José Amilcar T. Filho pelos serviços prestados ao Laboratório de Biologia Comparada e Abelhas (LBCA) e cuidados com a Coleção Entomológica "J.M.F. Camargo" (RPSP) a partir da qual foi obtida boa parte do material utilizado nesse estudo.

À Dra. Denise A. Alves e ao Dr. Sidnei Mateus por cederem a maior parte do material de Meliponina utilizado para as dissecções. Ao Dr. Túlio Nunes, pelos espécimes de meliponínios australianos. À Dra. Aline R. C. Andrade-Silva pelos espécimes de Euglossa melanotricha. Ao Msc. Diego Moure pelos espécimes de Centris utilizados para o estudo da cápsula cefálica das abelhas.

Ao Dr. Lars B. Vilhelmsen por ter me recebido durante o estágio em Copenhagen. Obrigado pelo auxílio com as dissecções das cabeças de abelhas e discussões sobre sobre a morfologia geral de Hymenoptera. 
Ao assistente e técnico de microscopia Dr. David K. B. Cheung pelas instruções para utilização do equipamento de microscopia eletrônica de varredura do Zoological Museum, Natural History Museum of Denmark.

Aos colegas Msc. Daniel Carmo e Dr. Danilo Ament pelos ensinamentos e discussões acerca dos softwares utilizados para análises cladísticas. Aos colegas Msc. Diego Aguilar Fachin e Msc. Fabio Batagini Quinteiro pelo auxílio na utilização dos softwares de edição de imagens e confecção de ilustrações vetoriais. Ao colega Msc. Daercio Lucena pelas dicas dadas na utilização do equipamento fotográfico do laboratório.

A todos os amigos dos grupos de discussão de Sistemática Filogenética e de Morfologia Comparada de Hexapoda pelas discussões sempre enriquecedoras e produtivas.

Aos companheiros, ex-companheiros e agregados do Laboratório de Biologia Comparada e Abelhas (LBCA): Daniel Carmo, Fábio Quinteiro, Fábio Oliveira, Daercio Lucena, Fabiano Stefanello, Anne Costa e Rafael Moretto. Obrigado pela amizade e por todos os bons momentos do nosso dia-a-dia.

Aos companheiros, ex-companheiros e agregados do Laboratório de Morfologia e Evolução de Diptera: Gabriela Pirani, Diego Fachin, Ana Motta, Caleb Martins, Alana Brunini, Danilo Ament, Renato Capellari, Paula Riccardi, Rafaela Falaschi, Mateus Henrique, Marco Marinho, Sarah Oliveira e Maria Isabel Balbi. Obrigado pela amizade e por todos os bons momentos e pequenas felicidades da vida.

A todos os meus amigos de apartamento, ex-moradores e agregados: Danilo Benette, Murilo Pastana, Rafael Teixeira, Matheus Teixeira, Paulo Ricardo, Pedro Rizzato, Vitor Zanchetta e Lorena Viana. Obrigado pela amizade, convivência diária e "opa-opa days" por todos esses anos.

Aos meus amigos de Santos: Caio Mendella, Cainã Sekiguchi, Diego Pinheiro, Davidson Sestaro, Eduardo Menezes, Érick Vecchiato, Eric Akira, Rafael Barreto, Lucas Ferraz, Guilerme Melo e todos os demais. Obrigado por mais de dez anos de pura amizade, carinho e compreensão, pois mesmo nos meus longos períodos de ausência se mantiveram os mesmos amigos/irmãos de sempre.

Aos meus queridos e inestimáveis pais: Douglas Porto e Kátia Denisse Sasso Porto. Meras linhas de agradecimento seriam fúteis, mas tento deixar aqui registrado o quanto aprecio todo o carinho, dedicação e confiança depositados em mim. Muito obrigado! À minha querida irmã, Gabriele Sasso Porto, por todo o amor e carinho, além das nossas conversas bobas esporádicas. 
TABLE OF CONTENTS

Abstract
Resumo
List of tables figures
Obenectives introduction

Chapter 1 - Comparative Morphology of the Mandibles and Head Structures of Corbiculate Bees (Hymenoptera: Apidae: Apini) …………………………………....10

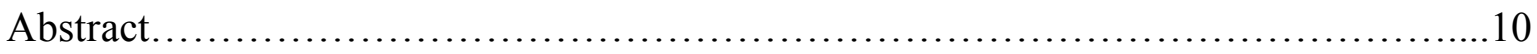

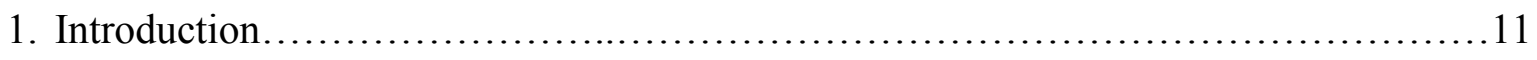

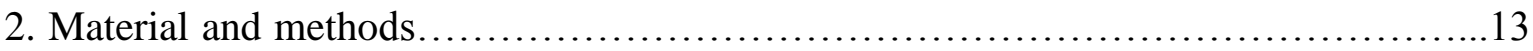

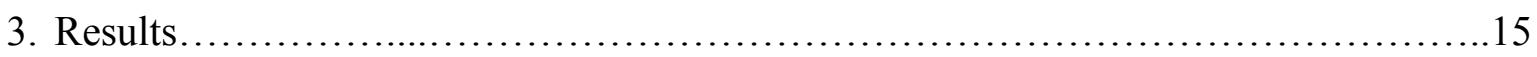

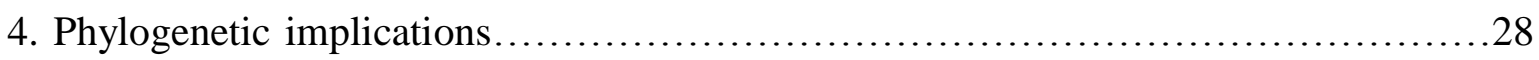

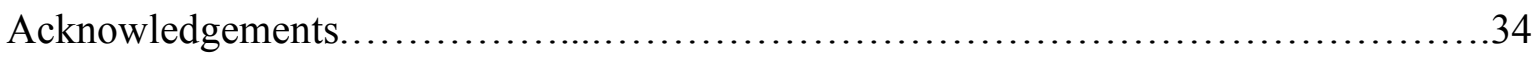

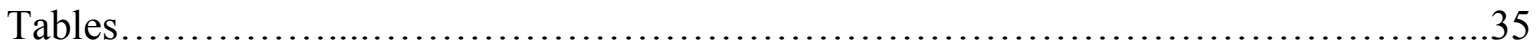

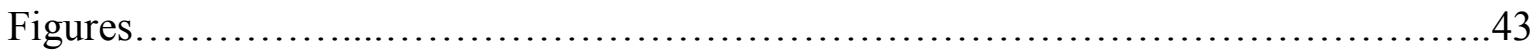

Chapter 2 - Comparative Morphology of Internal Structures of Mesosoma of Bees with emphasis in the Corbiculate Clade (Hymenoptera: Apidae: Apini) ..............67

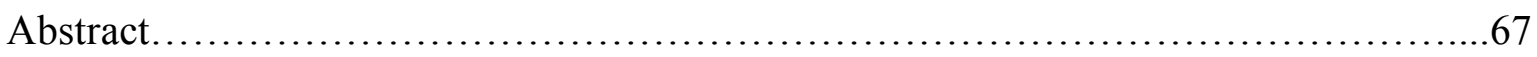

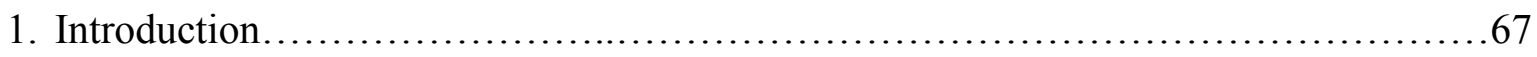

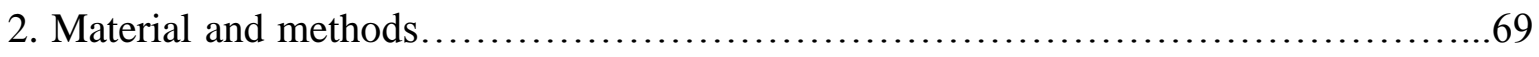

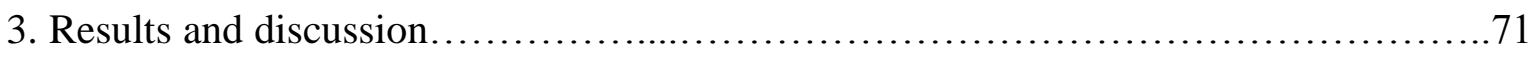

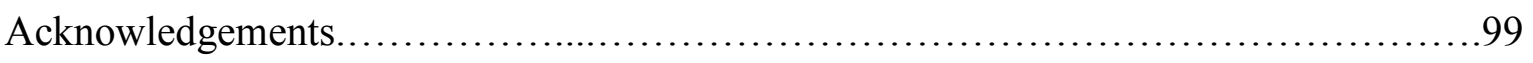

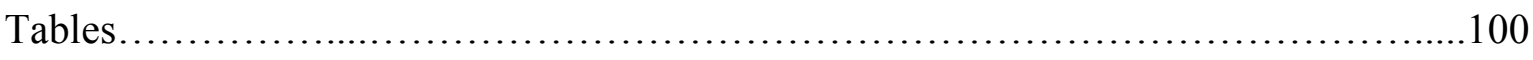

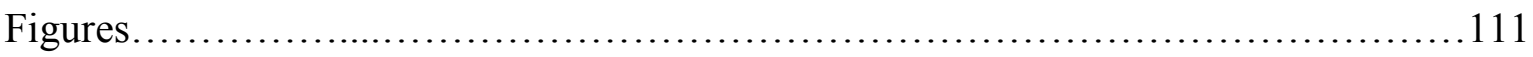


Chapter 3 - Phylogenetic analysis of corbiculate bees (Hymenoptera: Apidae: Apini) inferred with new data from internal structures of exoskeleton

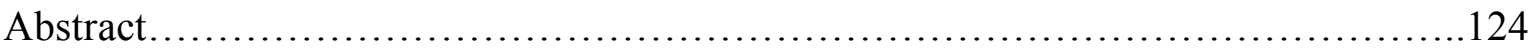

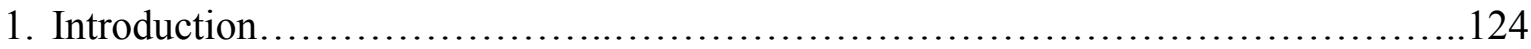

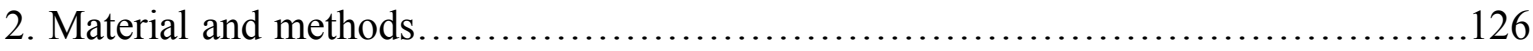

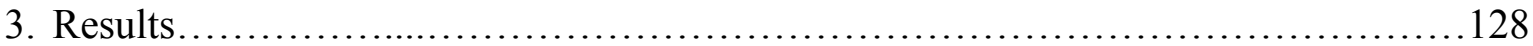

4. Discussion................................................................. 130

Acknowledgements............................................................. 137

Tables..................................................................... 138

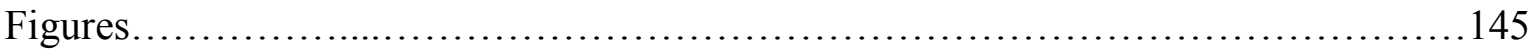

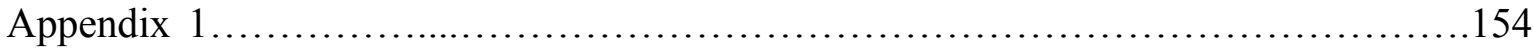

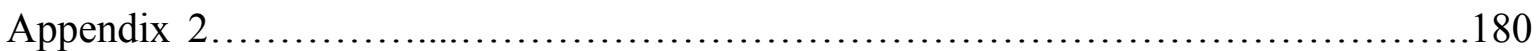

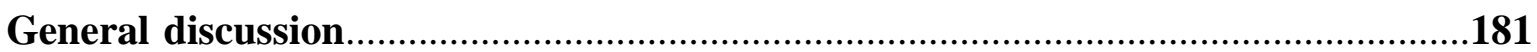

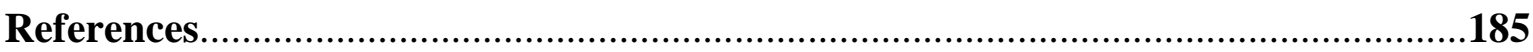

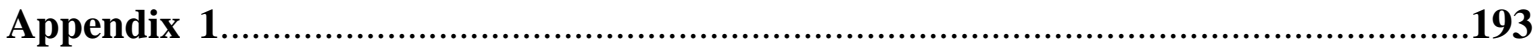




\section{Abstract}

Bees are aculeate hymenopterans, which, altogether, comprise approximately 20,000 valid species divided into seven families, among which Apidae is one of the best known groups. In addition to the species richness, a remarkable diversity of social behaviors is known to exist within this group, ranging from strict solitary taxa (over $80 \%$ of bee species) to groups showing highly sophisticated behaviors related to life in society. One of the most fascinating groups of bees in this respect is that of corbiculate bees (Apidae: Apinae: Apini). Amongst its four subtribes, Apina and Meliponina comprise the so-called "fixedcaste eusocial" groups. Phylogenetic relationships of corbiculate bees have been under intense controversy for the last two decades, thus hampering a robust understanding of the evolution of eusocial traits. Most hypotheses based on morphological/behavioral data point to a clade uniting Apina + Meliponina, thus favoring a single origin scenario for the evolution of "fixed-caste" eusociality. Molecular datasets, on the other hand, consistently indicated independent origins for this cluster of traits. In this contribution, the main goal was to re-evaluate the phylogenetic relationships between the major lineages of corbiculate bees exploring new sources of information from internal skeletal structures of exoskeleton. It were provided descriptions/discussions on some important morphological complexes of the head capsule, mandibles, sitophore, propectus, meso/metafurca and mesophragma of bees. Also, it was attempted to standardize the current morphological terminology used for bees to that used for other Hymenoptera. A set of different phylogenetic analysis with the main lineages of Apidae emphasizing the corbiculate clade was conducted. The complete morphological matrix with 93 characters from external morphology and 42 from internal structures of exoskeleton resulted into two most parsimonious trees with 376 steps, CI=45 and $\mathrm{RI}=78$, which were congruent with the current morphological/behavioral scenario for the corbiculate relationships: Euglossina $+($ Bombina + (Apina + Meliponina $)$ ). It was demonstrated that characters extracted from internal structures of exoskeleton, although poorly explored, can provide critical evidence to elucidate the "corbiculate controversy". Moreover, despite the inclusion of new sources of data is fundamental to elucidate this issue, some efforts should be made to reevaluate many characters currently in (re)use. Also, the inclusion of fossil information and use of new technologies for phenotypic studies can open new windows for future comparative morphological studies in bees.

Keywords: 1. Systematics 2. Comparative Morphology 3. Eusociality 4. Apina 5. Meliponina 


\section{Resumo}

Abelhas são himenópteros aculeados que, ao todo, compreendem aproximadamente 20.000 espécies válidas distribuídas em sete famílias, das quais Apidae é um dos grupos mais conhecidos. Além da diversidade de espécies, uma notável diversidade de comportamentos sociais é conhecida para esse grupo, desde táxons estritamente solitários (mais de $80 \%$ das espécies de abelhas) a grupos exibindo comportamentos altamente sofisticados relacionados à vida em sociedade. Um dos grupos mais fascinantes nesse aspecto é o das abelhas corbiculadas (Apidae: Apinae: Apini). Dentre suas quatro subtribos, Apina e Meliponina compreendem os chamados grupos eussociais de "casta-fixa". As relações filogenéticas entre abelhas corbiculadas têm estado sob intensa controvérsia nas duas últimas décadas, o que dificulta a compreensão robusta da evolução dos atributos da eussocialidade. A maioria das hipóteses baseadas em dados morfológicos/comportamentais apontam para um clado unindo Apina + Meliponina, portanto favorecendo um cenário de origem única para a eusocialidade de "castafixa". Conjuntos de dados moleculares, por outro lado, indicam consistentemente origens independentes para esse conjunto de atributos. Nesta contribuição, o objetivo principal foi reavaliar as relações filogenéticas entre as principais linhagens de abelhas corbiculadas, explorando novas fontes de informação das estruturas internas do exoesqueleto. Foram fornecidas descrições/discussões sobre alguns importantes complexos morfológicos da cabeça, mandíbulas, sitóforo, meso/metafurca e mesofragma de abelhas. Além disso, buscou-se padronizar a atual terminologia morfológica usada para abelhas com aquela usada para outros Hymenoptera. Um conjunto de diferentes análises com as principais linhagens de Apidae, enfatizando o clado das corbiculadas, foi conduzido. A matrix morfológica completa com 93 caracteres de morfologia externa e 42 de estruturas internas do exoesqueleto resultou em duas árvores mais parcimoniosas com 376 passos, $\mathrm{CI}=45$ e $\mathrm{RI}=78$, que são congruentes com o cenário morfológico/comportamental atual para as relações filogenéticas das corbiculadas: Euglossina $+($ Bombina $+($ Apina + Meliponina $)$ ). Foi demonstrado que caracteres de estruturas internas do exoesqueleto, apesar de pouco explorados, podem fornecer evidências críticas para elucidar a "controvérsia das corbiculadas". Ademais, apesar da inclusão de novas fontes de dados ser fundamental para essa questao, algum esforço deve ser feito para se reavaliar muitos caracteres atualmente em (re)uso. Além disso, a inclusão de informação de fósseis e uso de novas tecnologias para estudos fenotípicos podem abrir novas janelas para futuros estudos morfológicos em abelhas.

Palavras-chave: 1. Sistemática 2. Morfologia Comparada 3. Eussocialidade 4. Apina 5. Meliponina 


\section{LIST OF TABLES}

\section{Chapter 1}

Table 1. Species (Hymenoptera: Apoidea: Apidae) sampled for this work

Table 2. Morphological terminology utilized by different authors to make reference to some head capsule structures....

Table 3. Morphological terminology utilized by different authors to make reference to some mandible structure. . .40

Table 4. Matrix of character states distribution. . .42

\section{Chapter 2}

Table 1. Material examined 100

Table 2. Morphological terminology used previously by other authors to make reference to some structures of the mesosoma.... 103

Table 3. Matrix of character states distribution. 109

\section{Chapter 3}

Table 1. List of the material examined in this study.... .138

Table 2. Matrix of character state distribution used in the cladistics analysis of the morphological dataset

Table 3. Nodal support values of Figure 3 estimated with standard bootstrapping, symmetrical resampling, and Bremer support calculations 


\section{LIST OF FIGURES}

\section{General introduction}

Figure 1. Representation of four possible phylogenetic hypotheses explaining the relationships of corbiculate bees (Apidae: Apini) ..................................

\section{Chapter 1}

Figure 1. Images of heads in frontal view of cleared specimens .43

Figure 2. Explanatory figures based on structures pertaining to the head of Centris analis (Fabricius) . .44

Figure 3. Left mandibles....................................................45

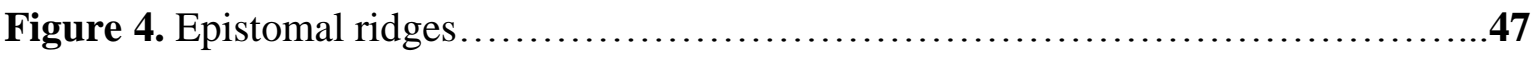

Figure 5. Occiput and postocciput............................................. 49

Figure 6. Hypostoma and secondary tentorial bridge.............................51

Figure 7. Paramandibular process of hypostoma..............................52

Figure 8. Tentorium and hypostoma....................................................................54

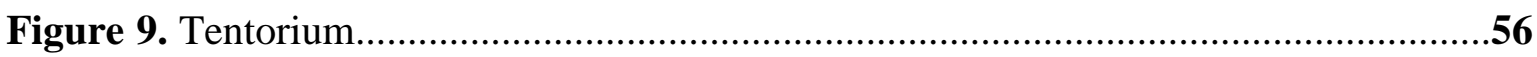

Figure 10. Sitophore and hypopharyngeal lobe...............................58

Figure 11. SEM images of the mandibles......................................59

Figure 12. SEM images of the occiput and postocciput...........................60

Figure 13. SEM images of the tentoria...............................................

Figure 14. SEM images of the dissected heads showing the tentorium................62

Figure 15. SEM images of the lower portion of clypeus depicting the pleurostomal condyle.

Figure 16. SEM images of the lower portion of clypeus depicting the paramandibular process

Figure 17. Mapping of character state changes onto concurrent phylogenetic hypotheses for corbiculate bees 65

Figure 18. Single tree obtained from cladistics analysis of the morphological dataset with characters from the head, mandibles and sitophore....

\section{Chapter 2}

Figure 1. Prosternum of Centris analis (Fabricius, 1804) 
Figure 2. Propleuron of Centris analis (Fabricius, 1804).

Figure 3. Mesofurcae/metafurcae and mesophragma of Centris analis (Fabricius, 1804)

Figure 4. Prosternum; ventral view

Figure 5. Prosternum; dorsal view

Figure 6. Propleuron; lateral view 116

Figure 7. Propleuron; ventral view 117

Figure 8. Cervical prominence

Figure 9. Mesofurca/metafurca; dorsal view.

Figure 10. Mesofurca/metafurca; anterior view.

Figure 11. Mesophragma.

Figure 12. Mesosomal character state changes on a phylogenetic hypothesis for bees...

\section{Chapter 3}

Figure 1. Three sets of analyses conducted with the morphological dataset.

Figure 2. Tree resultant from the additional analysis D with continuous data treated "as such"

Figure 3. Strict consensus obtained from the two most parsimonious trees resultant from analysis $\mathrm{B}$

Figure 4. Optimization of transformations of the morphological characters constructed for this work onto phylogenetic hypotheses for corbiculate bees commonly recovered with morphological, behavioral and molecular data 


\section{General introduction}

Bees comprise a much diverse group of aculeate hymenopterans included into the superfamily Apoidea (Melo, 1999), which also reunites the so-called "sphecoid wasps" (Sphecidae sensu Bohart \& Menke, 1976). Bees encompass approximately 20,000 valid species distributed into seven families: Andrenidae, Apidae, Colletidae, Halictidae, Megachilidae, Melittidae and Stenotritidae (Roig-Alsina \& Michener, 1993; Alexander \& Michener, 1995; Michener, 2007). The family Apidae, as defined by Roig-Alsina \& Michener (1993), is divided into three subfamilies: Xylocopinae (about 980 spp.), Nomadinae (about 1,200 spp.) and Apinae (approximately 3,500 spp.) (Ascher \& Pickering, 2014). The corbiculate bees correspond to one tribe of Apinae - the Apini - following the classificatory scheme suggested by A. Roig-Alsina (Roig-Alsina \& Michener, 1993; also followed by Silveira et al., 2002), subdivided into four subtribes: Apina (honey bees, genus Apis, 11 spp.); Bombina (bumblebees, genus Bombus, approximately 260 spp.); Euglossina (orchid bees, 5 genera, approximately $240 \mathrm{spp}$.) and Meliponina (stingless bees, about 50 genera, more than $500 \mathrm{spp}$.). One of the most remarkable aspects of this group is the fact that almost all its representatives - except the species of Euglossina and of the subgenus Bombus (Psithyrus) - exhibit behaviors characterized as eusocial (Noll, 2002).

The study of sociality in insects in general is a field that has always instigated several questionings. Eusocial behavior has been traditionally defined by the division of labor between the member of the colony, cooperative brood care and superposition of generations (Michener, 1969, 1974, 2007). Amongst the distinct groups of insects, the cluster of traits that define eusociality certainly evolved several times independently (e.g., Isoptera; Hymenoptera: Vespidae, Formicidae, Halictidae: Augochlorini, Halictus, Lasioglossum, Apidae: Allodapini, Apini) (Cardinal \& Danforth, 2011). Amongst bees, the tribe Apini is one of the best known groups with eusocial representatives - Apina, Bombina and Meliponina. In this tribe, the representatives of Euglossina exhibit a broad spectrum of behaviors associated with distinct kinds of social organization, commonly with solitary and communal species, but in some instances as in a few species of the genus Euglossa, it is observed some behavioral repertoires characteristic of "fixed-caste" eusocial structures (sensu Almeida \& Porto, 2014; Appendix 1) - division of labor and superposition of generations (Augusto \& Garófalo, 2009, 2011). As defined by Michener 
(1974), the eusocial groups can be divided accordingly to their social organization into two "kinds": (1) "primitively eusocial" ("totipotent-caste" sensu Almeida \& Porto, 2014), as occurs in Bombina, in which there is only weak or no morphological differentiation between the castes; queens are not completely dependent on workers to found new colonies; and new colonies are temporary, ending after each annual cycle; and (2) "advanced eusocial" ("fixed-caste" sensu Almeida \& Porto, 2014), as occurs in Apina and Meliponina, in which queens and workers exhibit conspicuous morphological differentiation, the formers being completely dependent on the latters in the founding process; in addition to perennial colonies that can last several years.

The share of morphological and behavioral characters between the members of the last two subtribes has aroused interest of many researchers and raised questions about the probable single origin of "fixed-caste" eusocial traits in Apini. Aware of this importance, several works in the last seven decades have focused on this matter trying to understand the internal relationships of corbiculate bees and their implications to the evolution of eusocial characters. To accomplish this purpose, it has been employed all the different sources of data to extract phylogenetic information: morphology, behavior and molecules (Cardinal \& Packer, 2007; Kawakita et al., 2008; Almeida \& Porto, 2014 and references cited in these works). The pioneer work of Michener (1944), although has not been based on a proper phylogenetic analysis, was one of the first to recover a clade comprised by Apina + Meliponina. Several posterior studies based on morphological datasets have also recovered this grouping (e.g., Michener, 1990; Prentice, 1991; Roig-Alsina \& Michener, 1991). Also, some of the reanalyzes in conjunction with molecular data or with fossil taxa included have also supported this clade - Figure 1. A, B (e.g., Chavarría \& Carpenter, 1994; Schultz et al., 1999; Ascher et al., 2001; Engel, 2001a, b; Cardinal \& Packer, 2007). Two works, have utilized exclusively behavioral data to construct their matrices: Noll (2002) and Canevazzi \& Noll (2015). They also weigh the body of evidence favoring the single origin hypothesis for the evolution of the clusters of eusocial traits in Apini, accordingly to the following relationships: Euglossina + (Bombina + (Apina + Meliponina)). These findings are in agreement with the majority of the previous hypotheses based solely in morphology.

However, as has been observed in the phylogenies published with molecular data in the last 20 years (Cardinal \& Packer, 2007), despite the existence of some divergences between them a commonly recovered clade is the grouping of Meliponina + Bombina - 
Figure 1. C, D. This alternative scenario implies that at least some of the traits associated with the "fixed-caste" eusociality would have arisen twice (e.g., Cameron, 1991, 1993; Koulianos et al., 1999; Cameron \& Mardulyn, 2001; Cardinal et al., 2010; Cardinal \& Danforth, 2011; Kawakita et al., 2008; Martins et al., 2014).
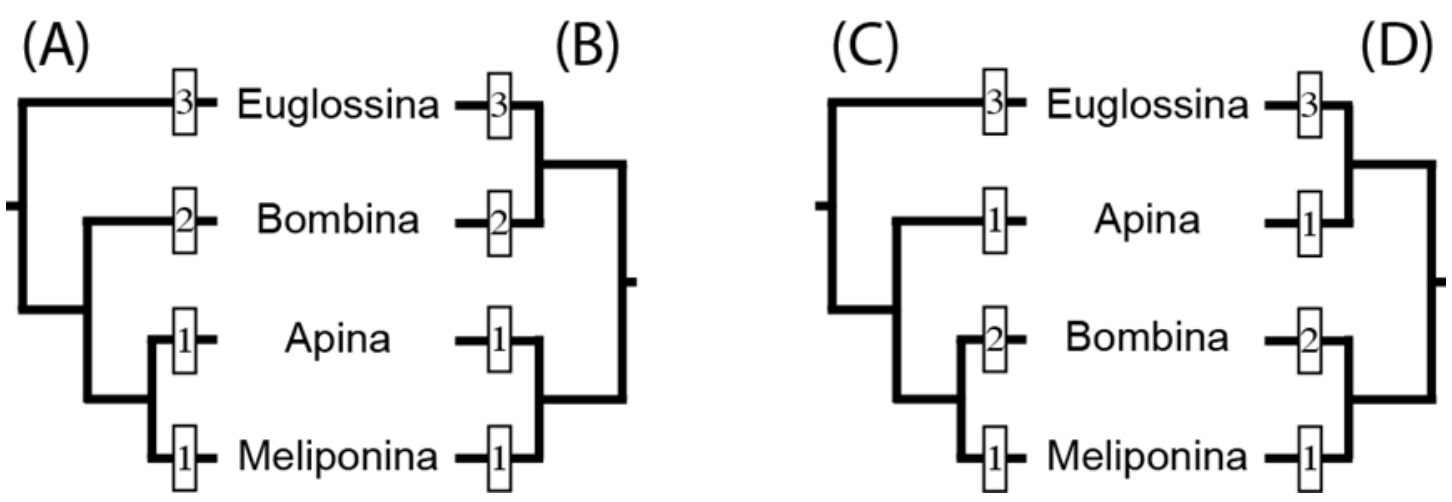

Figure 1. Representation of four possible phylogenetic hypotheses explaining the relationships of corbiculate bees (Apidae: Apini). Hypothesis $A$ is favored by the majority of morphological datasets generated to the present; hypothesis $B$ results from a few isolated morphological studies; hypotheses $C$ and $D$ are commonly encountered when analyzing molecular data. Numbers on each terminal indicate the "kind" of social organization present in the respective taxon; (1) "fixed-caste" eusociality; (2) "totipotent-caste" eusociality; (3) other kinds of social organization, ranging from solitary, communal to almost eusocial structures. Hypothesis A indicates a single origin for the "totipotent-caste" (Bombina + Apina + Meliponina) and "fixed-caste" eusocial traits (Apina + Meliponina). All the remainder hypotheses imply less parsimonious explanations for the evolution of these clusters of social traits in this group. Figure from Almeida \& Porto (2014).

The sharp disagreements between the different sources of data show that this still is an open question in literature. Important advances have been observed in the last decade, especially concerning the utilization of molecular data (Cameron \& Mardulyn, 2001; Kawakita et al., 2008; Cardinal et al., 2010; Cardinal \& Danforth, 2011, 2013; Martins et al., 2014). However, such advances were not followed equally by a morphological counterpart. The most comprehensive studies on external skeletal morphology of bees in a phylogenetic framework were the ones of Michener (1944), Roig-Alsina \& Michener (1993), Alexander \& Michener (1995), and Melo (1999). Several subsequent studies have reutilized or reinterpreted data already present in literature (e.g., Michener, 1990; Chavarría \& Carpenter, 1994; Schultz et al., 1999; Ascher et al., 2001; Payne, 2014). Few works have truly explored new sources of morphological data for phylogenetic studies (e.g., Serrão, 2001; Peixoto \& Serrão, 2001; Cardinal \& Packer, 2007). Taking this into account, the search for new sources of morphological information is indispensable to add evidence 
that can help to elucidate the "corbiculate controversy". In this context, poorly explored portions of the phenotype, as the internal structures of the exoskeleton - tentoria, phragmata, furcae, internal ridges and other kinds of apodemes - seems especially promising, as it have been demonstrated for other groups of Hymenoptera (e.g., Vilhelmsen, 1999, 2000a, b, c, 2011; Vilhelmsen et al., 2010). Also, it is important to reevaluate most of the characters currently in (re)use in literature in conjunction with an accurate examination of the viability of new sources of data to the comprehension of the relationships of corbiculate bees in particular, and bees in general.

\section{Objectives}

Thus, the main objective of this work was to reevaluate the phylogenetic relationships between the major lineages of corbiculate bees (Apidae: Apinae: Apini) utilizing a new set of morphological data never explored extensively for these taxa. Furthermore, tree additional questions were addressed:

(1) evaluate the importance of characters obtained from internal skeletal morphology, poorly explored in literature, to elucidate the phylogenetic relationships amongst bees.

(2) advance in the comprehension of the origin and evolution of eusocial behavior in corbiculate bees.

(3) analyze the congruence of molecular datasets in literature with the morphological dataset resultant from this work. 


\title{
Chapter 1 - Comparative Morphology of the Mandibles and Head Structures of Corbiculate Bees (Hymenoptera: Apidae: Apini)
}

\author{
DIEGO S. PORTO ${ }^{1,2^{*}}$, LARS VILHELMSEN ${ }^{2}$, EDUARDO A. B. ALMEIDA $^{1}$
}

${ }^{1}$ Laboratório de Biologia Comparada e Abelhas (LBCA), Departamento de Biologia, Faculdade de Filosofia, Ciências e Letras de Ribeirão Preto (FFCLRP), Universidade de São Paulo, Avenida Bandeirantes, 3900, 14040-901, Ribeirão Preto-SP, Brazil.

${ }^{2}$ Biosystematics, Natural History Museum of Denmark, University of Copenhagen, Universitetsparken 15, DK-2100 Copenhagen, Denmark.

* Corresponding author

Running title: Head morphology of bees

Keywords: bumblebees, honeybees, hypostoma, internal morphology, orchid bees, sitophore, stingless bees, tentorium.

Reproduced with kind permission of John Wiley \& Sons Inc., License Number 3770340577980. Reproduced from the publication: Porto, D.S., Vilhelmsen, L. \& Almeida, E.A. (2015). Comparative morphology of the mandibles and head structures of corbiculate bees (Hymenoptera: Apidae: Apini). Systematic Entomology.

\begin{abstract}
Phylogenetic relationships of corbiculate bees have been a well-known focus of controversies over the past 30 years. The majority of the morphological datasets supports the monophyly of Apina+Meliponina, whereas molecular datasets recovers Meliponina as sister to Bombina. This issue is especially critical to the proper understanding of the evolution of clusters of traits that define the eusocial behavior. This work provides a description and discussion on characters of the head capsule, mandibles, and sitophore of bees. Thirty-three characters are proposed and optimized onto concurrent phylogenetic hypotheses for corbiculate bees, which results in seven derived character-states supporting the monophyly of the Apina+Meliponina+Bombina and nine supporting the Apina+Meliponina clades. Although some striking synapomorphies (e.g., tentorial bridge,
\end{abstract}


pleurostomal condyle, hypopharyngeal lobe) support the former clade, most characters supporting the latter (i.e., Apina+Meliponina) were losses/reductions. Moreover, two previously undescribed character transformations on hypostoma and sitophore favor the Bombina+Meliponina clade. Internal head capsule characters are useful for phylogenetic analysis in comparative studies of bees, and corbiculate bees in particular, when efforts are made to solve the "corbiculate controversy". An attempt is made to standardize the current terminology used for bees and for other Hymenoptera.

\section{Introduction}

The corbiculate bees (Apidae: Apini) comprise one of the most intriguing and wellstudied subgroups of all bees. The corbiculate clade comprises the following four lineages, here treated as subtribes: Honeybees (Apina: genus Apis, 11 spp.), bumblebees (Bombina: genus Bombus, approx. 260 spp.), orchid bees (Euglossina: five genera, approx. 240 spp.), and stingless bees (Meliponina: ca. 60 genera, over 500 spp.) (Michener, 2007; Rasmussen \& Cameron, 2010; Camargo \& Pedro, 2013; Moure et al., 2012; Ascher \& Pickering, 2014). This is a clade that has attracted major scientific interest because of its importance for the understanding of social evolution (e.g., Michener, 1974; Cardinal \& Danforth, 2011), but also for economic importance (pollination, honey production). Given its importance, it is all the more frustrating that corbiculate phylogeny remains controversial, despite decades of research.

The phylogenetic relationships of corbiculate subtribes have been a well-known focus of controversies over the past 30 years, especially since the introduction of molecular data sets. This issue is especially critical to the proper understanding of the evolution of the clusters of traits that define "totipotent-caste" and "fixed-caste" eusociality (Cardinal et al., 2007; Kawakita et al., 2008; Almeida \& Porto, 2014). Several authors have attributed the incongruences between alternative phylogenetic hypotheses to different properties of morphological and/or molecular data sources, inappropriate analytical methods, rooting problems, and insufficient amounts of data (Chavarría \& Carpenter, 1994; Lockhart \& Cameron, 2001; Engel, 2001a; Cardinal \& Packer, 2007; Payne, 2013).

Accordingly, several approaches to resolve the "corbiculate controversy" have been attempted: "total evidence" analysis (Chavarría \& Carpenter, 1994; Schultz et al., 1999; Cameron \& Mardulyn, 2001; Ascher et al., 2001; Payne, 2013; Canevazzi \& Noll, 2015), inclusion of extensive molecular (Kawakita et al., 2008; Martins et al., 2014) and/or 
morphological (Roig-Alsina \& Michener, 1993; Engel, 2001a, b; Cardinal \& Packer, 2007) and behavioral (Noll, 2002; Canevazzi \& Noll, 2015) data, and alternative analytical methodologies (Payne, 2013). Whereas most of these efforts focused on molecular data, morphology has been relatively neglected in recent years. Except for Cardinal \& Packer (2007) there has been no attempt to revise or expand morphological data sets bearing on corbiculate bee phylogeny in the past two decades.

Morphological characters explored so far include mainly external skeletal structures, especially mouthparts (Michener \& Fraser, 1978; Winston, 1979; Michener, 1984; Michener \& Brooks, 1984; Plant \& Paulus, 1987), legs (Cane, 1979; Michener, 1981; Schönitzer, 1986), and wings (Winston \& Michener, 1977; Michener, 1990; Roig-Alsina \& Michener, 1993). Only a handful of works have sought truly novel character sources on external skeletal morphology (Packer 2003, 2004; Cardinal \& Packer, 2007) or internal anatomy (Peixoto \& Serrão, 2001; Serrão, 2001).

In face of this, it is of prime importance to search for new sources of morphological characters for phylogenetic studies and revaluate characters currently in use. Internal structures of the exoskeleton (e.g., tentorium, internal ridges, apodemes, phragmata, furcae), although comparatively extensively studied in other hymenopteran groups (Vilhelmsen, 1996, 1999, 2000a, b, c, 2011; Beutel \& Vilhelmsen, 2007; Vilhelmsen et al., 2010; Karlsson \& Ronquist, 2012) were, to a certain extent, neglected in bees, either by the intrinsic difficulty of studying complex internal structures or by the lack of appropriate methods for imaging them (Deans et al., 2012). Only a few general works have discussed internal structures in more detail (Michener, 1944; Snodgrass, 1935, 1956; Camargo et al., 1967; Roig-Alsina \& Michener, 1993; Melo, 1999). Roig-Alsina \& Michener (1993) were the first to propose a large number of characters from internal structures of the exoskeleton and to use them extensively in phylogenetic analysis of long-tongued bees. However, there are additional internal skeletal morphological complexes that are potential sources of phylogenetic information.

Another issue that deserves attention is relative discrepancies in current terminology used for bees and for other Hymenoptera. An exemplary attempt to broaden the application of bee morphology was that of Rightmyer (2004), which presented a welcome comparison between the morphological terminology applied for the sting apparatus of bees and general terminology for the hymenopteran ovipositor. A similar effort is made in the present work for internal structures of the head exoskeleton, and 
corbiculate bees (Apidae: Apini) are used as the basis for this comparative morphological research.

The main objective of this work was to perform a comparative study of mandibles and internal skeletal structures of head of the corbiculate bees (Fig. 1) with special emphasis on the under-explored portions of the internal head anatomy. It was also attempted to reevaluate internal characters previously proposed by other authors as well as the potential of these structures as sources of information to illuminate the controversy concerning the phylogenetic relationships in corbiculate bees.

\section{Material and methods}

\subsection{Material examined}

Females of nine species of corbiculate bees and two outgroup centridine taxa were selected to represent the range of morphological variation across the study group. In the case of eusocial species, only workers were studied. 1-3 exemplars of each species were dissected. Specimens for this study were obtained from Coleção Entomológica "J.M.F.Camargo" (RPSP), Departamento de Biologia, FFCLRP-USP and donated from individuals listed in the Acknowledgements who kindly provided part of the material. The tribal classification adopted here follows that of Roig-Alsina (in Roig-Alsina \& Michener, 1993), which recognizes each traditionally accepted tribe as subtribes, and the whole corbiculate clade is named Apini. The complete list of species sampled for the comparative morphological investigation is presented in Table 1. Abbreviations of genus-names when used were done with the two initial letters in some cases to avoid ambiguities.

\subsection{Whole mounts (WM)}

Ethanol preserved specimens were treated with a $10 \% \mathrm{KOH}$ solution heated to approximately $90^{\circ} \mathrm{C}$ for 20 to 45 minutes to dissolve muscle fibers and clear the cuticle. Heads were then removed and dissected using surgical forceps, scalpels and eyelens scissors to remove the mandibles (with the apodemes) and expose the tentoria. Dissected parts were stored in glycerin and studied under a dissection stereomicroscope. 


\subsection{Scanning Electron Microscopy (SEM)}

Dissected specimens were transferred to absolute ethanol through a series of dehydrating solutions and critical-point dried. They were then mounted on small metal stubs with double-adhesive tape and coated with a mixture of platinum-palladium before being examined in a JSM-6335F Field Emission Scanning Electron Microscope.

\subsection{Illustrations and photography}

Line drawings of internal structures were made with the aid of a camera lucida coupled to a Leica M10 dissection stereomicroscope. The drawings were then digitalvectored with Adobe Illustrator CS2 software. Images of the whole heads were taken using a Leica DFC450 camera coupled to a Leica MZ16 dissection stereomicroscope and postedited in Adobe Photoshop CS4 software.

\subsection{Morphological descriptions and terminology}

Full names of skeletal structures (followed by the respective abbreviations in parenthesis) are presented only the first time they appear in the plates named "explanatory figures", which serve as a guide and a glossary for the structures and views treated comparatively in plates to follow. The morphological terminology referring to general structures of head and mandibles of bees follows Michener (1944), Michener \& Fraser (1978), and Roig-Alsina \& Michener (1993). An attempt to homologize these terms with general terms used in other Hymenoptera is made using the works of Snodgrass (1910, 1942), Vilhelmsen (2011) and the HAO web portal (Yoder et al., 2010).

\subsection{Character coding and interpretation.}

All character state transformations were optimized on topologies that express opposing views on the phylogenetic relationships among the corbiculate subtribes: Apina as sister-group of Meliponina ('Hypothesis 1', supported by most morphological and behavioral studies: Michener, 1944; Maa, 1953; Michener, 1990; Prentice, 1991; RoigAlsina \& Michener, 1993; Chavarría \& Carpenter, 1994; Schultz et al., 1999; Ascher et al., 2001; Engel, 2001a, b; Noll, 2002; Cardinal \& Packer, 2007; Payne, 2013; Canevazzi \& Noll, 2015), and Bombina as sister-group of Meliponina ('Hypothesis 2', supported by Cameron \& Mardulyn, 2001). In either scenario, the Euglossina were placed as sister to the remaining corbiculate subtribes, and Apini was deliberately represented as monophyletic 
and sister to Centridini. A third optimization was evaluated having Apina as sister to Euglossina and Meliponina as sister to Bombina ('Hypothesis 3'), which represents the scenario most frequently recovered with molecular data (Cameron, 1991; Koulianos et al., 1999; Schultz et al., 1999; Cameron \& Mardulyn, 2001; Kawakita et al., 2008; Cardinal et al., 2010; Cardinal \& Danforth, 2011; Cardinal \& Danforth, 2013). Character states were optimized onto the different tree topologies with WinClada (Nixon, 2002), depicting the unambiguous transformations and ambiguous character-state changes optimized as delayed transformations (DELTRAN). The choice of the latter optimization scheme (as opposed to an ACCTRAN scheme) is arbitrary, but it was applied here to reflect a conservative view about the transformations of the character-states in consideration, avoiding unnecessary interpretations of homology in these uncertain cases.

\subsection{Phylogenetic methods}

To evaluate the phylogenetic signal of the characters from head capsule, mandibles and sitophore independently of other morphological complexes, an additional cladistic analysis was performed with the matrix presented in this work. An equal weighs phylogenetic analysis was conducted using the exhaustive tree search procedures implemented in the software TNT v1.1 (Goloboff et al., 2008), with all discrete characters treated as non-additive.

\section{Results}

\subsection{Morphological annotations}

The following sections contain general comments on the internal skeletal morphology of some head structures and the mandibles of corbiculate bees. For more detailed treatments on internal morphology of bees see the works of Snodgrass $(1910,1942$, 1956), Michener (1944), and for mandibles, see Michener \& Fraser (1978). An attempt to standardize the terminology used for morphological descriptions of internal and some external characters of head capsule and mandibles of bees and correlate these terms to more general ones used for other hymenopteran groups is made in Tables 2 and 3. 


\subsection{Mandibles (Figs 2A, 3A-K, 11A-K)}

Michener \& Fraser (1978) comprehensively treated the mandibular morphology of bees. A remarkable feature of the mandibles of corbiculate bees is the conspicuously spoon-shaped or spatulate apex. This is the result of an expanded apex of the pollex, with more than one tooth, and a wide blunt-like tooth of the rutellum, especially evident in $B$. brasiliensis Lepeletier (Figs 3D, 11D), Ap. mellifera Linneaus (Figs 3K, 11K), and the observed meliponine taxa (Figs 3E-J, 11E-J). Although the spatulate apex is also somewhat evident in Ep. flava Friese (Figs 3A, 11A), the apex of the mandible of this species is not exactly spatulate, but has a produced tooth of rutellum, which is not as blunt as in most corbiculate species. The number of pollex teeth also seems to be constant in most observed species. Just in C. analis (Fabricius) (Figs 3B, 11B), which has one well-developed tooth and one much reduced, and in Ap. mellifera (Figs 3K, 11K), which has no teeth at all, this condition is different; in all other observed corbiculate bees the apex of the pollex has at least two teeth developed. An expanded apex of the pollex with more than one tooth is probably plesiomorphic for the corbiculate clade and perhaps for the corbiculate-centridine clade as well. Amongst the corbiculate bees, the shape of pollex teeth is also variable. In the outgroup species Ep. flava (Figs 3A, 11A) and C. analis (Figs 3B, 11B), as it is in the corbiculate Eg. melanotricha Moure (Figs 3C, 11C); and also in most of the other euglossine genera (D.S.Porto, unpubl. observations), the pollex teeth are large and welldeveloped in relation to the tooth of the rutellum. In B. brasiliensis (Figs 3D, 11D) and meliponine taxa (Figs 3E-J, 11E-J), these teeth are considerably reduced, less than the half of the width of the tooth of rutellum, and in Ap. mellifera (Figs $3 \mathrm{~K}, 11 \mathrm{~K}$ ), they are absent.

Another conspicuous feature of the mandibles in these groups is the degree of development of the various external mandibular grooves/ridges/carinae. According to Michener \& Fraser (1978), the common condition for most bees is the presence of three sets of grooves and the associated ridges/carinae on the external surface of mandibles: the acetabular groove (and carina), the outer groove (and ridge), and the condylar groove (and carina). These grooves and ridges/carinae are variable in the corbiculate taxa. In Ap. mellifera (Figs 3K, 11K) and all studied Meliponina (Figs 3E-J, 11E-J), the outer and condylar grooves and their respective ridges/carinae are absent. In the meliponine taxa, however, the acetabular groove and an oblique groove are present on the external surface of mandible. If the latter groove is homologous to the outer groove of mandible in other bees is difficult to decide. It follows, though, a trajectory that is not exactly the same as the 
outer groove in other groups, thus it may be considered as a secondary development in the meliponine taxa. On the other hand, Eg. melanotricha (Figs 3C, 11C) and B. brasiliensis (Figs 3D, 11D) have the full set of grooves/ridges/carinae and have the distal part of the outer groove subdivided into an upper and a lower branch.

\subsection{Epistomal ridge and lower portion of clypeus (Figs 2B, 4A-K, 14A-C, 15A-E,} 16A-D)

The epistomal ridge is an internal structure corresponding to the external epistomal sulcus. It has three main parts: a dorsal and two latero-ventral ones. The dorsal portion of the epistomal ridge extends between the insertions of the anterior tentorial arms (indicated externally by the anterior tentorial pits). The latero-ventral parts of the epistomal ridge extend from the insertions of the anterior tentorial arms, to the ventral ridge of the clypeus which sometimes bends laterally and continues above the mandibular foramina.

The point of insertion of the anterior tentorial arm on epistomal ridge is situated more dorsally in bees than in most other Hymenoptera (Michener, 1944). In all groups examined, the anterior tentorial pits are positioned high on the epistomal sulcus, but separated by at least half of the antennal foramen diameter from the dorso-lateral angles of the clypeus. The only exception is B. brasiliensis (Fig. 4D), in which the anterior tentorial pits are exactly on the dorso-lateral angles.

Another variable trait within the corbiculate clade is the degree of development and extension of the median expansion of epistomal ridge. This structure results from the expansion of the epistomal ridge (probably together with the anterior attachment of the thickening of secondary tentorial bridge) to form a sheet laterad to the anterior tentorial pit. This expansion is reduced and restricted to the area adjacent to anterior tentorial pit in Ep. flava (Fig. 4A), B. brasiliensis (Fig. 4D), Scaptotrigona depilis (Moure) (Fig. 4I), and Ap. mellifera (Fig. 4K). In all other observed taxa, the median expansions are conspicuously developed for a considerable length of the latero-ventral portion of the epistomal ridges. The ventral ridge of the clypeus (apical inflection of clypeus, Roig-Alsina \& Michener, 1993: char. 17) is wide, approximately half of antennal foramen diameter in outgroups (Fig. 4A-B), and in Eg. melanotricha (Fig. 4C). In most of the other corbiculate species a reduced ridge seems to be the general condition (Fig. 4D,F,I,J,K), although among the Meliponina, some species have a more or less wide ridge (Fig. 4E,G-H), but it can be an allometric consequence of the reduced size of these specimens. 
A cluster of morphological modifications that may be functionally correlated is found on the lateral margins of the inferior portion of the clypeus. In Ep. flava (Fig. 4A), $C$. analis (Figs 4B, 14B), and Eg. melanotricha (Fig. 4C) the clypeus is strongly protruded anteriorly, probably associated with a large proboscis, at least in Euglossina. In these taxa, and also in B. brasiliensis (Figs 4D, 15C), the lateral margins of ventral portion of the clypeus are strongly bent posteriorly, which does not occur in other examined corbiculate species (Figs 4E-K, 15E, 16D). In Ep. flava (Figs 4A, 15A), C. analis (Figs 4B, 14B), and especially in Eg. melanotricha (Figs 4C, 15B), these margins are also expanded laterally, partly covering the pleurostomal condyle, the point of articulation of the head capsule to the mandibular acetabulum. Another conspicuous modification of the epistomal ridge is the posterior extension of the ventral portion, visible through the ventral margin of the compound eye, present in the outgroups (Figs 8A-B, 14B, 15A) but absent in the corbiculate taxa (Fig. 8C-K).

\subsection{Occiput, postocciput and subforaminal bridge (Figs $2 \mathrm{G}, 5 \mathrm{~A}-\mathrm{K}, 12 \mathrm{~A}-\mathrm{K}$ )}

The external occipital sulcus of Michener (1944) corresponds to the insertion of the fan-shaped sheet of the posterior tentorial arm on the head capsule. As noted by Michener (1944), this sulcus is normally identifiable when a well-developed fan-shaped sheet of the posterior tentorial arm is present, although there are cases in which a sulcus is observed with a poorly-developed fan-shaped sheet, as in Melipona scutellaris Latreille (Figs 5J, 12J). However, the term "occipital sulcus" is a more general term, already in use for early diverging hymenopteran lineages, which describes a sulcus that extends from the posterior tentorial pit towards the lateral ocellus, being represented internally by the occipital ridge (HAO, Yoder et al., 2010; see also Vilhelmsen 1999). Thus, the new term "arched sulcus of occiput" is used here to refer to the "occipital sulcus" of Michener (1944). This sulcus is present in Ep. flava (Figs 5A, 12A), C. analis (Figs 5B, 12B), Eg. melanotricha (Figs 5C, 12C), B. brasiliensis (Figs 5D, 12D), and M. scutellaris (Figs 5J, 12J).

Another term that causes some confusion is the "hypostomal bridge", together with "postgenal bridge" and "postoccipital bridge", widely used by previous authors (e.g., Snodgrass, 1942; Michener, 1944). Snodgrass (1942) stated that the region of the head capsule between the occipital foramen and oral foramen is composite in nature. The "postoccipital bridge" according to Snodgrass is the continuation of the postocciput beneath the occipital foramen. This area is notoriously difficult to interpret. Vilhelmsen 
(1999) considers the postoccipital bridge to be an internal structure. The closing of the postocciput beneath the occipital foramen, forming what will be called herein the postoccipital bridge, following Snodgrass (1942), seems to be a condition found in most Apocrita. However, the area immediately beneath the postoccipital bridge is more complex. At least for bees, it seems that the median projections of the postgena, the postgenal lobes, are not connected, thus a true postgenal bridge is not completely formed. The median region of this area receives an extension of the hypostoma, forming what Snodgrass (1942) called as "hypostomal bridge". It is not clear if he referred only to this extension as the "hypostomal bridge" or if he included also the posterior connection of both hypostomata on the roof of proboscidial fossa. As the fossa is present in most bees, the term hypostomal bridge will be used to refer to the entire region, as in Michener (1944). An alternative view is that the median area beneath the postoccipital bridge can constitute an in situ sclerotisation of the hypostomal area, in contrast to the postgenal bridge, which is presumably formed by 'invasion' from the postgenal lobes, as mentioned above.

To avoid confusion and to stress to the composite nature of the bridge beneath postoccipital bridge, the term "subforaminal bridge" (Snodgrass, 1942) will be adopted. The whole surface of the subforaminal bridge is weakly sclerotised and, in most cases, more or less concave. When this concavity is very deep, it forms what Roig-Alsina \& Michener (1993) termed the "postoccipital pouch", as seen in Eg. melanotricha (Figs 5C, 12C), and other euglossine taxa (pers. observations). In other taxa, e.g., Ep. flava (Figs 5A, 12A), B. brasiliensis (Figs 5D, 12D), and Trigonisca nataliae (Moure) (Figs 5G, 12G) this area is very broad, but only slightly concave. In all taxa observed this region can easily be identified. It is uncertain if this area is only postoccipital in origin or includes the hypostomal extension, but the term "postoccipital pouch" will be retained as defined in Roig-Alsina \& Michener (1993).

\subsection{Hypostoma (Figs 2D-F, 6-8A-K, 14A-C, 16A-D)}

The hypostoma of bees is conspicuously modified and greatly expanded due to the formation of a deep proboscidial fossa. The hypostoma forms almost the entire roof of the fossa in some species, and is mostly delimited anteriorly by an arched and heavily sclerotised margin with the maxillary processes of the hypostoma situated laterally. Antero-laterally, beyond the maxillary processes, the hypostoma becomes nearly vertical and forms, on each side of head, a sheet that almost closes the mandibular foramen, 
separating it from the proboscidial fossa. The anteriormost point of this sheet is sometimes conspicuously produced anteriorly, nearly abutting internally against the clypeus, forming the paramandibular process of the hypostoma. Posterolaterally, the hypostoma is delimited by the hypostomal carina that, in many bees, extends out of the proboscidial fossa, forming a considerable portion of the median area of the subforaminal bridge.

There is considerable discussion concerning the nature of the entire subforaminal bridge in different hymenopteran taxa (Snodgrass, 1942; Vilhelmsen 1999, 2011; Burks \& Heraty 2015), and a few comments were made above. As stated by previous authors (Snodgrass, 1942; Michener, 1944) both hypostomata are always connected by the hypostomal bridge in bees, thus the singular term hypostoma is correctly used in this case.

The degree of anterior extension of the hypostomal bridge and concomitant fusion to the secondary tentorial bridge are variable in the examined taxa. In some species as Ep. flava (Fig. 6A), B. brasiliensis (Fig. 6D), and all the meliponine representatives (Fig. 6E-J), the hypostomal bridge occupies a considerable portion of the proboscidial fossa and is fused to the secondary tentorial bridge for between a third and a half of the length of the latter. In C. analis (Fig. 6B), Eg. melanotricha (Fig. 6C), and Ap. mellifera (Fig. 6K) the fusion at most reaches a quarter of the length of tentorium; in the former species, there is almost no fusion at all. Another variable feature concerning the hypostomal bridge is the width of its connection to the secondary tentorial bridge. In the latter three species, the connection is largely restricted to the median area of the secondary tentorial bridge. In $B$. brasiliensis (Fig. 6D) the connection is broader, but still on the median area, whereas in Ep. flava and all the meliponine representatives the connection is complete, the line of fusion between the sheets of secondary tentorial bridge and hypostoma being located far laterally when looking on the proboscidial fossa in ventral view.

Though the maxillary process in bees is conspicuously displaced anteriorly on the hypostoma when compared to the condition in other hymenopterans, this is even more pronounced in Ep. flava (Fig. 8A), C. analis (Fig. 8B), and to an extreme degree, in Eg. melanotricha (Fig. 8C). All other studied exemplars have the maxillary processes positioned more medially on the hypostoma (Fig. 8D-K). A well-developed paramandibular process (Figs 2F, 7A-D,14A,C, 16A-B), strongly projected and reinforced by a longitudinal carina, the paramandibular carina, seems to be the plesiomorphic condition for corbiculate bees, as it is found in the outgroups (Figs 7A-B, 14A, 16A), Eg. melanotricha (Figs 7C, 14C), and B. brasiliensis (Figs 7D, 16B). 


\subsection{Tentorium (Figs 2C-E, 8-9A-K, 13, 14C)}

The tentorium of bees, as stated by Michener (1944), is a complex structure that is strongly modified when compared to the same structure in other hymenopterans. These changes are associated with the evolution of complex folding mechanisms of the proboscis and the development of a deep proboscidial fossa in bees. The tentorium is composed of the paired tentorial arms connected posteriorly by the transverse tentorial bridge (primary tentorial bridge of Michener, 1944). Each tentorial arm is composed of a well-developed anterior tentorial arm, which forms the main component of the tentorium, a dorsal tentorial arm, and a posterior tentorial arm.

The topological delimitation between the tentorial components depends on the position of the tentorial bridge. The anterior tentorial arm is the part of the tentorium between the anterior connection to internal surface of head capsule and the tentorial bridge. The posterior tentorial arm is very short and corresponds to the small portion between the tentorial bridge and the posterior connection to the wall of head capsule. Proximate to the junction of the tentorial bridge and the anterior tentorial arm, a slender rod-like apodeme, the dorsal tentorial arm, is inserted and follows an oblique trajectory until almost meeting the internal margin of antennal rim anteriorly.

In bees, and probably in most Apocrita, the dorsal tentorial arms are very thin, but in most non-apocritan taxa these arms are well developed and constitute a considerable portion of the tentorium (Vilhelmsen 1996). The anterior tentorial arms, which in bees are well developed, are composed by two fused thickenings twisted relative to each other (Fig. 2E). One of these thickenings follows a more or less straight line from the anterior to the posterior tentorial pit and seems to correspond to the ancestral position of the main axis formed by the anterior and posterior tentorial arms. The other thickening initiates its trajectory anteriorly from a point just below the antennal foramen, above and medial to the first thickening, and follows an oblique trajectory towards the posterior internal surface of head, meeting the first thickening and then becoming ventral to it. This second thickening gradually expands medially, from its anterior connection to the internal surface of head capsule. The connection is demarcated externally by the inner subantennal groove. As the second thickening extends posteriorly, it gives rise to another median thickening, the thickening of secondary tentorial bridge. The thickenings of the secondary tentorial bridges meet at the posterior portion of the tentorium to form the secondary tentorial bridge. 
The thickenings of the tentorium in bees are connected throughout by sclerotised sheets, and the anterior sheet formed between them, below the antennal foramen, is termed by Roig-Alsina \& Michener (1993) the dorsal sheet of anterior tentorial arm, demarcated externally by the outer subantennal groove. Roig-Alsina \& Michener (1993: figs 1-3) used the term "eutentorial arm" (figs) to refer to the thickening of the tentorium that extends in a straight line between the anterior and posterior tentorial pits; we follow this terminology.

In all corbiculate bees and most other bee taxa the secondary tentorial bridge is directly connected to the hypostomal bridge. Although this connection is complete in all groups studied here, there is some variation concerning the width of the connection between the sclerotised sheets of the secondary tentorial bridge and the hypostomal bridge, as discussed in the previous section. The eutentorial arm is further expanded dorsally in some specimens, forming a sheet between the dorsal sheet of anterior tentorial arm and the insertion of the dorsal tentorial arm (Fig. 8A,E-F,J,K). This sheet seems to be a dorsal expansion of the eutentorial arm, beyond the point where the latter meet with the other tentorial thickening, and not a posterior expansion of the dorsal sheet of anterior tentorial arm, as itself is limited dorsally by the latter thickening. This expansion is evident in Ap. mellifera (Fig. 8K), M. scutellaris (Fig. 8J), and to a lesser degree, in Austroplebeia australis (Friese) (Fig. 8F) and Tetragonula carbonaria (Smith) (Fig. 8E). A similar condition also occurs in Ep. flava (Fig. 8A), but it seems to be a secondary development, since the whole tentorium is somewhat heavily sclerotised in this specimen and apparently modified due to the partial anterior displacement of the maxillary process of hypostoma.

The posterior portion of the tentorial arm and its connection to the surface of the head capsule beyond the tentorial bridge is another complex region. It probably mainly corresponds to the posterior tentorial arm. The attachment to the internal surface of head capsule, referred to as the posterior root of the tentorium by Michener (1944), forms a dorso-laterally expanded sheet, delimiting a ventral cavity between it, the posterior tentorial arm, and the postoccipital ridge. This sheet is the so-called posterior fan-shaped sheet of the tentorium (Roig-Alsina \& Michener, 1993), and here will be referred to as the fan-shaped sheet of the posterior tentorial arm. The development of this sheet is quite variable, being very small in Ap. mellifera (Figs 9K, 13K) and all the examined Meliponina (Figs 9E-J, 13E-J) and wide in the other taxa (Figs 9A-D, 13A-D), delimiting a broad ventral cavity. 
Another important character that has been largely ignored by previous authors, but is remarkable for some corbiculate bees, is the shape of the tentorial bridge. In most bees, and almost all other hymenopterans, a straight bridge seems to be the plesiomorphic condition (Figs 5 A-C, 9A-C, 13A-C). In Ap. mellifera (Figs 5K, 9K, 13K), B. brasiliensis (Figs 5D, 9D, 13D), and especially in the meliponine taxa (Figs 5E-J, 9E-J, 13E-J), the bridge is conspicuously arched ventrally, probably associated with the antero-ventral displacement of the tendon of the tentorio-hypopharyngeal muscle (=ventral cibarial dilatator) (for muscle terminology, see Vilhelmsen, 1996; HAO).

The complex anterior connection of the tentorium to the head wall (anterior root of tentorium; Michener, 1944) makes it difficult to accurately understand some characters proposed by previous authors. Roig-Alsina \& Michener (1993) stated that the fusion of the eutentorial arm, the dorsal sheet of anterior tentorial arm, and the attachment of the thickening of secondary tentorial bridge to the interior surface of head capsule, indicated externally respectively by the anterior tentorial pit and the outer and inner subantennal grooves, delimits a small triangular area below the antennal foramen in some taxa (RoigAlsina \& Michener, 1993: char. 14, state 1). This appears to be the condition found in a few specimens as Eg. melanotricha (Fig. 4C), B. brasiliensis (Fig. 4D), Te. carbonaria (Fig. 4E), and Ap. mellifera (Fig. 4K). In C. analis (Fig. 4B), the attachment of the thickening of secondary tentorial bridge is displaced ventrally to the level of epistomal ridge (Fig. 2B), leaving a broad open area beneath the antennal foramen. In all other studied specimens (Fig. 4A,F-J), there is almost no space between these landmarks, thus a small triangular area seems absent (Roig-Alsina \& Michener, 1993: char. 14, state 0).

Another observation made by Roig-Alsina \& Michener (1993) concerns the width of the lateral expansion of the thickening of secondary tentorial bridge above the epistomal ridge (their char. 16). This expansion is wide and well-developed in Ep. flava (Fig. 4A), $C$. analis (Fig. 4B), Eg. melanotricha (Fig. 4C), and B. brasiliensis (Fig. 4D). In Ap. mellifera (Fig. 4K) and all the meliponine representatives studied (Fig. 4E-J) this expansion is very short and inconspicuous, but this can be probably a result of the reduced size of the head of many specimens (most meliponine taxa) and the close proximity of the lower margin of antennal foramen to the epistomal ridge. In Eg. melanotricha (Figs 4C, 14C) and other euglossine taxa (pers. observations) a thin lateral spur projects from the dorsal sheet of anterior tentorial arm reaching to the compound eye margin at level of antennal foramen. 
The presence of this structure seems to be a synapomorphy for the Euglossina, as noted by Roig-Alsina \& Michener (1993).

\subsection{Sitophore (=pharyngeal plate) (Fig. 10A-J)}

The sitophore, commonly referred as the "oral plate" or "pharyngeal plate" by previous authors (Snodgrass, 1910, 1942; Michener, 1944), is a plate-like sclerite present on the proximal portion of the hypopharyngeal surface, proximal to the functional mouth. Its proximal margin receives the insertion of the tentorio-hypopharyngeal muscle (ventral cibarial dilatator, Vilhelmsen, 1996; HAO). The plate is produced dorso-posteriorly, forming two sclerotised bars, the pharyngeal rods, flanking a less sclerotised median area. Near their proximal ends, the pharyngeal rods receive the insertion of muscles connected to the frons, e.g., the retractors of the mouth angle and the lateral suspensors of the mouth (Vilhelmsen, 1996) or, alternatively, the muscle frontobuccalis anterior (M. 45 in Beutel \& Vilhelmsen, 2007) and muscle tentoriobuccalis lateralis (M49 in Beutel \& Vilhelmsen, 2007), respectively. The point of insertion of these muscles is demarcated by a small protuberance, which in the meliponine representatives (Fig. 10F-J) is considerably displaced from the apex of the rod.

The openings of the hypopharyngeal glands are located on the antero-lateral corners of the sitophore; they are especially distinct in Ap. mellifera (Fig. 10A). These openings are small, but also distinct, in all other observed corbiculate taxa (Fig. 10D-J).

The hypopharyngeal lobe is located on the distal margin of the sitophore, covering the infrabuccal pouch. The apex of hypopharyngeal lobe is quite variable amongst the observed taxa. In Ep. flava (Fig. 10B), C. analis (Fig. 10C), and Eg. melanotricha (Fig. 10D) it is considerably elongated, with a bilobed apex in the former two species and a simple apex in the latter. In Ap. mellifera (Fig. 10A), the hypopharyngeal lobe presents a pentagonal shape (triangular fold of Snodgrass, 1942), with a deeply cleft bifid apex. In all the studied meliponine representatives, this lobe is more or less triangular, with a trilobate apex (Fig. 10E-J). In B. brasiliensis (Fig. 10D) the condition seems to be intermediate between the elongated morphotype of outgroups and the trilobate morphotype of meliponine taxa. 


\section{8. Character list}

The morphological study presented above resulted in the following list of 33 characters from external/internal structures of the head capsule, mandibles and sitophore (=pharyngeal plate). The complete matrix of character states distributions utilized for the optimizations and cladistic analysis (see the next section) is given in Table 4.

\section{Mandibles}

1. Mandibular apex (modified from Roig-Alsina \& Michener, 1993: char. 60): (0) conical (Fig. 3A-B); (1) spatulate (Fig. 3C-K).

2. Preapical tooth (tooth of pollex): (0) absent (Fig. 3K); (1) present (Fig. 3A-J).

3. Pollex teeth: (0) with first tooth much reduced and second tooth extremely developed (Fig. 3B); (1) teeth equally developed (Fig. 3A,C-J).

4. Pollex teeth: (0) well-developed, approximately as wide as the tooth of rutellum (Fig. $3 \mathrm{~A}-\mathrm{C})$; (1) reduced, less than the half of the width of the tooth of rutellum (Fig. 3D-J).

5. Acetabular groove of mandible: (0) absent (Fig. 11K); (1) present (Fig. 11A-J).

6. Condylar groove of mandible: (0) absent (Fig. 11E-K); (1) present (Fig. 11A-D).

7. Outer groove of mandible: (0) absent (Fig. 11E-K); (1) present (Fig. 11A-D).

8. Distal path of outer groove of mandible: (0) not subdivided (Fig. 11A-B); (1) subdivided into upper and lower rami (Fig. 11C-D).

9. Oblique groove of mandibles: (0) absent (Fig. 11A-D,K); (1) present (Fig. 11E-J).

\section{Head capsule}

10. Anterior tentorial pit (modified from Roig-Alsina \& Michener, 1993: char. 2): (0) low on epistomal sulcus; positioned between 0.5 and 1.0 times antennal foramen diameter from the dorso-lateral angle of clypeus (Fig. 4A-C,E-K); (1) high on epistomal sulcus; positioned less than 0.5 times antennal foramen diameter from the dorso-lateral angle of clypeus (Fig. 4D).

11. Median expansion of epistomal ridge adjacent to the insertion of eutentorial arm to head capsule: (0) short; length less than 1.0 times antennal foramen diameter (Fig. 4A,D,I,K); (1) long; length more than 1.5 times antennal foramen diameter (Fig. 4B-C,E$\mathrm{H}, \mathrm{J})$. 
12. Ventral ridge of clypeus (modified from Roig-Alsina \& Michener, 1993: char. 17): (0) broad, at least 0.33 times antennal foramen diameter (Figs 4A-C,E,G-H, 14A); (1) reduced to a small strip, less than 0.33 times antennal foramen diameter (Figs 4D,F,I-K, 16C).

13. Lateral margins of ventral portion of clypeus (modified from Roig-Alsina \& Michener, 1993: char. 6): (0) only slightly deflected posteriorly; angled less than 30 degrees (Figs 4E$\mathrm{K}, 15 \mathrm{E}, 16 \mathrm{D}$ ); (1) strongly deflected posteriorly; angled between 60 and 90 degrees (Figs 4A-D, 14A, 15C, 16A)

14. Clypeus: (0) markedly protruded anteriorly; forming a distinct convex surface (Fig. 14B); (1) not strongly protruded anteriorly; forming only slightly convex surface (Figs 15C,E, 16D).

15. Pleurostomal condyle (modified from Roig-Alsina \& Michener, 1993: char. 5): (0) contiguous with the lateral margin of ventral portion of clypeus (Fig. 15C-E); (1) partly covered by the lateral margin of ventral portion of clypeus (Figs 14B, 15A-B).

16. Posterior extension of the ventral portion of epistomal ridge visible through the ventral margin of compound eye: (0) absent (Fig. 8C-K); (1) present (Figs 8A-B, 14B, 15A).

17. Arched sulci of occiput: (0) absent (Figs 5E-I,K, 12E-I,K); (1) present (Figs 5A-D, 12A-D,J).

18. Ventral portion of arched sulci of occiput: (0) reaching the level of the posterior tentorial pits (Figs 5A,B,D, 12A-B,D); (1) terminating before reaching the level of the posterior tentorial pits (Figs 5C,J, 12C,J).

19. Surface of subforaminal bridge (modified from Roig-Alsina \& Michener, 1993: char. 21): (0) shallow or only moderately concave; postoccipital pouch small (Figs 5A-B,D-K, 12A-B,D-K); (1) very deep; postoccipital pouch large and distinct (Figs 5C,12C).

20. Fusion of hypostomal bridge to the secondary tentorial bridge (modified from RoigAlsina \& Michener, 1993: char. 23): (0) restricted to the median area of hypostomal bridge (Fig. 6C,K); (1) forming a wide median sheet distinguishable by two lateral fusion lines (Fig. 6A,D-J).

21. Connection of hypostomal bridge to tentorium: (0) restricted to the posterior fourth of tentorial length or less (Fig. 6B-C,K); (1) extending for more than the posterior fourth of tentorial length (Fig. 6A,D-J).

22. Maxillary process of hypostoma: (0) located medially on hypostoma (Fig. 8D-K); (1) displaced anteriorly on hypostoma, nearly abutting against clypeus (Fig. 8A-C). 
23. Paramandibular process of hypostoma (modified from Melo, 1999: char. 12): (0) anteriorly produced; almost abutting the internal surface of clypeus (Figs 7A-D, 14A,C, 16A-B); (1) not produced; terminating far away from the internal surface of clypeus (Figs 7E-K, 16C-D).

24. Paramandibular carina: (0) present (Figs 7A-D, 14A,D,J); (1) absent (Fig. 7E-K).

\section{Tentorium}

25. Lateral spur projecting from the dorsal sheet of anterior tentorial arm reaching the compound eye margin at level of the antennal foramen (modified from Roig-Alsina \& Michener, 1993: char. 13): (0) absent (Fig. 4A-B, D-K); (1) present (Figs 4C; 14H).

26. Attachment of the thickening of secondary tentorial bridge to the internal surface of head capsule (modified from Roig-Alsina \& Michener, 1993: char. 14): (0) not reaching the ventral margin of antennal foramen, delimiting a broad subantennal area (Fig. 4B); (1) nearly reaching the inferior margin of antennal foramen, delimiting a small triangular area (Fig. 4C-E, K); (2) reaching the inferior margin of antennal foramen, leaving no space between the thickening and the foramen (Fig. 4A, F-J).

27. Lateral expansion of the attachment of the thickening of secondary tentorial bridge above epistomal ridge (modified from Roig-Alsina \& Michener, 1993: char. 16): (0) less than 0.5 antennal foramen diameter (Fig. 4E-K); (1) more than or equal to 0.5 antennal foramen diameter (Fig. 4A-D).

28. Fan-shaped sheets of posterior tentorial arms (modified from Roig-Alsina \& Michener, 1993: char. 22): (0) reduced; not delimiting broad cavities opened ventrally (Figs 8-9E-K, 13E-K); (1) well-developed; delimiting broad cavities opened ventrally (Figs 8-9A-D, 13A-D).

29. Tentorial bridge seen through occipital foramen: (0) straight (Figs 5A-C, 9A-C, 13AC); (1) arched ventrally (Figs 5D-K, 9D-K, 13D-K).

\section{Sitophore}

30. Attachment point of frontal muscles on pharyngeal rods: (0) distinctly produced (Fig. 10B-E); (1) relatively small (Fig. 10A,F-J).

31. Position of the attachment point of frontal muscles on pharyngeal rod: (0) at less than a fourth of the total length from its apex (Fig. 10A-E); (1) at more than a fourth of the total length from its apex (Fig. 10F-J). 
32. Hypopharyngeal lobe: (0) elongate; at least 0.75 times the length of the pharyngeal rods (Fig. 10B-E); (1) short; equal to or less than 0.5 times the length of the pharyngeal rods (Fig. 10A,F-J).

33. Apex of hypopharyngeal lobe: (0) unilobate (Fig. 10D); (1) bilobate (Fig. 10A-C); (2) trilobate (Fig. 10E-J).

\section{Phylogenetic implications}

Hypotheses 1-3 (Fig. 17) represent, respectively, a synthesis of the main phylogenies previously published based on morphological/behavioral (Hypothesis 1) and molecular datasets (Hypotheses 2 and 3; see also the work of Cardinal et al., [2007] for a summary of concurrent hypotheses for corbiculate phylogeny on literature). When a delayed transformation (DELTRAN) scheme is applied to character state changes, the spatulate mandibular apex (char. 1:1), subdivided distal path of outer groove of mandible (char. 8:1) and a small triangular area delimited by the attachment of the thickening of secondary tentorial bridge to the internal surface of head capsule (char. 26:1) support the monophyly of corbiculate bees in all hypotheses considered. When a cladistic analysis was undertaken with the full morphological dataset (Fig. 18), the former two character state transformations (chars 1:1, 8:1) support unequivocally the monophyly of Apini whereas the latter (char. 26:1) constitute a homoplastic change under DELTRAN optimization. Also, the absence of the posterior extension of the ventral portion of epistomal ridge visible through the ventral margin of compound eye (char. 16:0) appears as another synapomorphy. At least for the eusocial species (e.g., B. brasiliensis, Ap. mellifera, and meliponine species), the spatulate apex (char. 1:1) could be related to other biological features: the development of a "true" worker caste, and the growing need for carrying/manipulating cell-building materials, cell construction and larvae provisioning. The subdivision of the outer groove of mandible (char. 8:1) is present only in $B$. brasiliensis, Eg. melanotricha, but also in other Bombina and Euglossina observed (pers. observations) and could be plesiomorphic for corbiculate bees (Fig. 17, Hypothesis 1) or have arisen twice (Fig. 17, Hypotheses 2, 3). The condition of the attachment of the thickening of secondary tentorial bridge (char. 26) is quite variable amongst the studied taxa; hence, the ancestral condition for corbiculate bees is hard to assess. Also, the presence of the posterior extension of the ventral portion of epistomal ridge (char. 16:1) could be viewed alternatively as a putative synapomorphy for Centrini as it was not 
observed in other apine taxa studied (pers. observations). The spatulate mandibular apex (char. 1:1) is the only character state change in this dataset that seems to correspond unambiguously to a derived condition for the corbiculate clade, since it is not observed in other closely related non-corbiculate taxa (e.g., Centridini; pers. observations). The lack of characters from the head capsule and mandibles that support the corbiculate taxa monophyly is not surprising, considering that the majority of the apomorphic character states for this clade are mainly linked to the metatibia/basitarsus modifications (e.g., corbicula, rastellum, basitarsal shape/articulation), and additional changes on the legs (e.g., basitibial plate), mesosoma (e.g., propleura, mesoscutellum), metasoma (e.g., pygidial plate), and mouthparts (e.g., maxillary palpus) (Michener, 1990; Roig-Alsina \& Michener, 1993). Roig-Alsina \& Michener (1993) suggested the expanded pollex (char. 60, state 1) as a synapomorphy for their "apine-clade", but this seems to be due to the presence of more than one pollex tooth, a condition that also occurs in Ep. flava in our dataset.

Another possible synapomorphy for corbiculate bees are the small, pedunculate hypopharyngeal glands, discharging through a single duct, which is reduced or absent in other bees (Cruz-Landim, 1967; Michener, 1990). The opening of this duct is on the anterolateral corners of the sitophore (=pharyngeal plate), as noted by Snodgrass (1942) for Ap. mellifera. We have observed similar openings located at the same place in the other corbiculate bees included (Fig. 10A,D-J) and absent in Eg. flava (Fig. 10B) and C. analis (Fig. 10C). However, the presence of gland openings was not included in the morphological matrix, because we have not observed enough genera of Euglossina, and the opening is difficult to identify in small meliponine specimens.

The posterior extension of the ventral portion of the epistomal ridge visible through the ventral margin of compound eye (char. 16:1), as mentioned above, probably can be considered a synapomorphy for Centridini, since this condition is present only in these taxa and has not been observed in other apid specimens (pers. observation). The condition presented by the apex of the hypopharyngeal lobe (char. 33) is quite variable in the examined taxa, and more specimens should be examined. In Hypothesis 3 (Fig. 17), when the characters were treated under the DELTRAN optimization, the markedly protruding clypeus (char. 14:0), the partly covered pleurostomal condyle (char. 15:1) and the anteriorly displaced paramandibular process of hypostoma (char. 22:1) are additional homoplastic character state transformations present on both Centridini species. 
One of the most remarkable outcomes of the present study was the great number of character state changes from head capsule, mandibles and sitophore that support the ApinaMeliponina-Bombina clade (Fig. 17, Hypotheses 1 and 2; Fig. 18; chars 12:1, 14:0, 15:0, 22:0, 29:1, 32:1). Although most earlier morphological datasets also corroborate this grouping (e.g., Michener, 1990; Roig-Alsina \& Michener, 1993, Engel, 2001a, b), it is important to note that most of the previous studies included only a small fraction of head capsule data on their matrices (excluding mouthparts). The only exception is Roig-Alsina \& Michener (1993), which included almost $19 \%$ (25 characters) of external/internal head capsule characters in their matrix of adults (131 characters), although they included only four corbiculate representatives.

The persistent strong support for the Apina-Meliponina-Bombina clade from morphological data is in conflict with the results from most molecular datasets (e.g., Kawakita et al., 2008; Cardinal et al., 2010; Cardinal \& Danforth, 2011; Martins et al, 2014), which favors the placement of Apina as sister to Euglossina (Fig. 17, Hypothesis 3). The choice of phylogenetic hypothesis has far-reaching consequences for the understanding of evolution of a number of traits correlated with eusociality in the corbiculate bees. It seems that too much attention, however, was given to the issue of single/dual origin of "obligate" (i.e., fixed-caste, sensu Almeida \& Porto, 2014) eusociality to the detriment of answering some equally important questions: why is Apina grouping with Euglossina in almost all molecular hypotheses published so far if there is little morphological support for this grouping? Why are molecular and morphological datasets so incongruent in this specific case? There is no morphology only phylogeny published we are aware of that recovers a grouping of Apina-Euglossina (Schultz et al., 1999; Cardinal et al., 2007; Kawakita et al., 2008; Almeida \& Porto, 2014). The combined analysis of Cameron \& Mardulyn (2001) retrieved the Apina-Meliponina-Bombina clade, but with Meliponina and Bombina as sisters (Fig. 17: Hypothesis 2).

Only having the fusion area of the hypostomal bridge to the secondary tentorial bridge restricted to the median area of hypostomal bridge (char. 20:0) seems to support Apina + Euglossini (hypothesis 3, Fig. 17). However, it is not totally clear if this can be the result of the restricted connection of the tentorium to the hypostomal bridge, limited to the posterior third of the tentorial length (char. 21:0). On the other hand, the present study found six unequivocal character state changes in the head capsule, mandible, and sitophore that support the Apina-Meliponina-Bombina clade (chars 12:1, 14:1, 15:0, 22:0, 29:1 and 
32:1) in Hypotheses 1-2 (Fig. 17) and in the tree retrieved by the cladistic analysis conducted (Fig. 18): the ventral ridge of clypeus reduced to a small strip (char. 12:1), the only weakly protruding clypeus (char. 14:1), the pleurostomal condyle contiguous with the lateral margin of inferior portion of clypeus (char. 15:0), the maxillary process of hypostoma located medially (char. 22:0), the arched tentorial bridge (char. 29:1), and the shortened hypopharyngeal lobe (char. 32:1). This evidence adds to the already large amount of morphological data to make this grouping even more strongly supported. Also, the reduced pollex teeth in relation to the tooth of rutellum (char. 4:1) is additional support for Hypothesis 1 (Fig. 17) and in the tree obtained from the parsimony analysis (Fig. 18).

If one excludes the possibility that Apina is the sister-group of Bombina, which has received support in only a few very small morphological datasets (Plant \& Paulus, 1987; Peixoto \& Serrão, 2001) and one behavioral dataset (Pereira-Martins \& Kerr, 1967), it seems that the "corbiculate controversy" boils down to identifying the sister group of Meliponina: Apina or Bombina? Our data set favors Apina as the sister group of Meliponina (Fig. 17, Hypothesis 1; Fig. 18), as this grouping is supported by nine unequivocal character state changes (chars 6:0, 7:0, 13:0, 17:0, 23:1, 24:1, 27:0, 28:0, 30:1). However, most of these (chars $6,7,17,27,28,30$ ) are loss or reduction characters, thus requiring a careful inspection in some cases. For example, the loss of different mandibular grooves (chars 6:0 and 7:0) in Apina and Meliponina might be explained by other ecological constraints related to the activities of cell-building and manipulation of construction materials. Moreover, it would be interesting to compare the mandible of workers to that of the queens in obligate eusocial species, as it was observed that at least some grooves are developed in latter (Snodgrass, 1942; Michener \& Fraser, 1978). The three sets of grooves, however, were treated as separate characters, since for the meliponine taxa, the acetabular groove is present and the homology of the oblique groove to the outer groove is uncertain (Michener \& Fraser, 1978). The only character that does not constitute a loss/reduction is the degree of posterior bending of the lateral margins of the ventral portion of the clypeus (char. 13:1). It may be correlated to the weakly convex clypeus of the Apina and Meliponina representatives, although it not seems to be the case in $B$. brasiliensis, whose clypeus is not as protuberant as in E. melanotricha and the outgroups, but also presents strongly bent lateral margins of the ventral portion of clypeus. Likewise, some of the characters included here, as the presence of the arched sulcus of occiput (char. 17:1) and the development of the fan-shaped sheets of posterior tentorial 
arms (char. 28:1) may not be independent (e.g., chars 17 and 28), thus inflating the evidence favoring the Apina-Meliponina clade. However, it is important to note that Michener (1944) already stated that there are species in which the arched sulci of occiput are present, despite the reduced fan-shaped sheet of posterior tentorial arms, as seen in $M$. scutellaris (Figs 5J, 9J, 12J, 13J).

The evidence favoring the Bombina-Meliponina clade (Fig. 17, Hypothesis 2) consists of one unequivocal (char. 33:2) and one homoplasious (char. 21:1) character state changes. The former is the trilobate apex of the hypopharyngeal lobe. Although the condition presented in the meliponine representatives is very similar among the studied specimens, the trilobate apex of B. brasiliensis is slightly different. Thus, it would be desirable to see more Bombina species to confirm the occurrence of this condition. The extended connection of the tentoria-hypostoma (char. 21:1) is uniting Bombina-Meliponina, but this condition is also present in Ep. flava and is variable in other Euglossina than Eg. melanotricha (DSP, unpubl. obs.), which suggests this may be plesiomorphic for corbiculate bees.

In all hypotheses considered (Fig. 17) and in the most parsimonious tree resulting from the cladistics analysis undertaken (Fig. 18), the monophyly of Meliponina is supported by the presence of a conspicuous oblique groove on the external surface of the mandibles (char. 9:1) and the position of the point of attachment of frontal muscles on the pharyngeal rods far from its apex (char. 31:1). The relationships among meliponine representatives, however, were mostly unresolved using the dataset presented here (Fig. 17, Hypothesis 1 and Fig. 18 only differ in respect to the phylogenetic relationships within the Meliponina).

Some characters used are apparently too invariable to resolve relationships at the generic level. For instance, the lateral spur projecting from the dorsal sheet of anterior tentorial arm (char. 25:1) is autapomorphic for Eg. melanotricha, but was also observed in representatives of almost all other euglossine genera (e.g., Exaerete, Eulaema, Eufriesea; D.S.P., unpubl.obs.), and is thus invariable for this clade. The presence of well-developed fan-shaped sheets of posterior tentorial arms (char. 28:1) seems to be a general condition for bees and its reduction (char. 28:0) in Apina-Meliponina is also invariable, as it is observed in other additional members of this clade (e.g., Apis dorsata Fabricius; Dactylurina staudingeri (Gribodo); Lophotrigona canifrons (Smith); Trigona hyalinata (Lepeletier); Melipona quadrifasciata Lepeletier). In the same way, the arched tentorial 
bridge (char. 29:1) is constant for all other Apina-Meliponina-Bombina specimens observed (D.S.P. unpubl. obs.). This indicates that some internal head capsule characters are useful for phylogenetic analysis at the tribal level, but might be too conserved for lower level relationships.

The overwhelming majority of morphological hypotheses recovered Apina as the sister of Meliponina (Fig. 17, Hypothesis 1; Fig. 18), whereas molecular datasets consistently recovers the clade Bombina-Meliponina (Fig. 17; Hypotheses 2-3). The inclusion of more data is expected and desirable, but it is also important to critically reevaluate the information being used, especially for morphological datasets. Most of the morphological analyses published after Roig-Alsina \& Michener (1993) recycled their character sample without carefully evaluating or adding much to it (e.g., Chavarría \& Carpenter, 1994; Schultz et al., 1999; Ascher et al., 2001; Engel, 2001a, b; Payne, 2013). Thus, it is important not only include new data, but also check and recheck previously used characters, especially those of difficult interpretation, and illustrate/image them as much as possible to avoid future ambiguities.

Another point that contributes to the already inflated "corbiculate controversy" is the discussion of the single/dual origin of "obligate eusociality" (fixed-caste eusociality, sensu Almeida \& Porto, 2014) in the Apina-Meliponina-Bombina clade. It might be plausible to suggest that the cluster of traits that define fixed-caste eusociality could have arisen more than one time in corbiculate bees. It is obvious that fixed-caste eusociality have arisen more than one time in the evolution of Hymenoptera (Cardinal \& Danforth, 2011) and it was demonstrated that probably the ancestral corbiculate species would have exhibited some features characteristic of "highly organized social structures" (Cardinal et al., 2010; Cardinal \& Danforth, 2011). It is important to consider that there exists much plasticity in the behaviors observed in some euglossine species, especially in the genus Euglossa (Garófalo et al., 1998; Cameron, 2004; Augusto \& Garófalo, 2009, 2011). On the other hand, the two biggest behavioral datasets used so far recover the Apina-Meliponina clade (Noll, 2002; Canevazzi \& Noll, 2015).

The inclusion of new sources of data is fundamental to solve the "corbiculate controversy", but some efforts should be made to reevaluate many characters currently used. More traditional techniques previously employed in morphological investigations can now be supplemented with new techniques (high resolution multifocus images, magnetic resonation imaging, and microCT scanning), which certainly will improve our 
understanding of complex internal anatomical structures and open new windows to explore them in a comparative context.

\section{Acknowledgements}

We would like to thank Denise A. Alves, Sidnei Mateus, Túlio Nunes, and Diego Moure for kindly providing valuable material used in this study. We thank also David K. B. Cheung for helping with the SEM execution. We would like to thank also two anonymous reviers for valuable comments and sugestions on the first draft of this manuscript. This project was partly supported by grant \# 2011/09477-9, São Paulo Research Foundation (FAPESP) to E.A.B.Almeida, and by FAPESP fellowships 2012/22261-8 and 2014/100900 to D. S. Porto. The software TNT was made available through the sponsorship of the Willi Hennig Society and Kevin Nixon is acknowledged for making Winclada available. 
Table 1. Species (Hymenoptera: Apoidea: Apidae) sampled for this work.

\begin{tabular}{|c|c|}
\hline Taxa & Species \\
\hline Apini: Apina & Apis mellifera Linnaeus, 1758 \\
\hline Apini: Bombina & Bombus (Fervidobombus) brasiliensis Lepeletier, 1836 \\
\hline Apini: Euglossina & Euglossa (Euglossa) melanotricha Moure, 1967 \\
\hline \multirow[t]{6}{*}{ Apini: Meliponina } & Austroplebeia australis (Friese, 1898) \\
\hline & Melipona (Michmelia) scutellaris Latreille, 1811 \\
\hline & Scaptotrigona depilis (Moure, 1942) \\
\hline & Tetragonisca angustula (Latreille, 1811) \\
\hline & Tetragonula carbonaria (Smith, 1854) \\
\hline & Trigonisca nataliae (Moure, 1950) \\
\hline \multirow[t]{2}{*}{ Centridini } & Centris (Heterocentris) analis (Fabricius, 1804) \\
\hline & Epicharis (Epicharana) flava Friese, 1900 \\
\hline
\end{tabular}


Table 2. Morphological terminology utilized by different authors to make reference to some head capsule structures. * = terms not explicitly proposed by the authors in those works, but used in morphological descriptions or figure legends; ${ }^{* *}=$ changes of terminology or new terms introduced to make correspondence to more general terms already used in other hymenopteran groups. Abbreviations: CDM, C. D. Michener (1944); HAO, Hymenoptera Anatomy Ontology; LV, L.Vilhelmsen (2011); RA\&M, Roig-Alsina \& Michener (1993); RES, R.E.Snodgrass (1910 and 1942).

\begin{tabular}{|c|c|c|c|c|c|c|}
\hline RES'10 & RES'42 & CDM & RA\&M & $\mathbf{L V}$ & HAO & This Work \\
\hline $\begin{array}{l}\text { Membranous } \\
\text { socket* }\end{array}$ & $\begin{array}{l}\text { Antennal } \\
\text { socket }\end{array}$ & $\begin{array}{l}\text { Antennal } \\
\text { socket }\end{array}$ & $\begin{array}{l}\text { Antennal } \\
\text { socket }\end{array}$ & $\begin{array}{l}\text { Antennal } \\
\text { foramen }\end{array}$ & $\begin{array}{l}\text { Antennal } \\
\text { foramen }\end{array}$ & $\begin{array}{l}\text { Antennal } \\
\text { foramen** }\end{array}$ \\
\hline 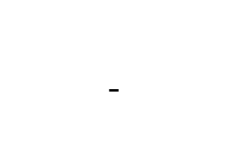 & $\begin{array}{l}\text { Rim of } \\
\text { antennal } \\
\text { socket* }\end{array}$ & $\begin{array}{l}\text { Antennal } \\
\text { sclerite }\end{array}$ & $\begin{array}{l}\text { Antennal } \\
\text { sclerite }\end{array}$ & - & Antennal rim & $\begin{array}{l}\text { Antennal } \\
\text { rim** }\end{array}$ \\
\hline- & Antennafer & Antennifer & Antennifer & - & Antennifer & Antennifer \\
\hline- & $\begin{array}{l}\text { Anterior arm } \\
\text { of tentorium }\end{array}$ & $\begin{array}{l}\text { Anterior arm } \\
\text { (of } \\
\text { tentorium)* }\end{array}$ & $\begin{array}{l}\text { Anterior } \\
\text { tentorial arm }\end{array}$ & - & $\begin{array}{l}\text { Anterior } \\
\text { tentorial arm }\end{array}$ & $\begin{array}{l}\text { Anterior } \\
\text { tentorial arm }\end{array}$ \\
\hline- & - & $\begin{array}{l}\text { Anterior root } \\
\text { (of } \\
\text { tentorium)* } \\
\text { (=connection } \\
\text { of anterior } \\
\text { arm to head } \\
\text { wall) }\end{array}$ & - & - & - & $\begin{array}{l}\text { Anterior root } \\
\text { of tentorium** }\end{array}$ \\
\hline $\begin{array}{l}\text { Smaller pit } \\
\text { (b, Fig. 9) }\end{array}$ & $\begin{array}{l}\text { Anterior } \\
\text { tentorial pit* }\end{array}$ & $\begin{array}{l}\text { Anterior } \\
\text { tentorial pit }\end{array}$ & $\begin{array}{l}\text { Anterior } \\
\text { tentorial pit }\end{array}$ & - & $\begin{array}{l}\text { Anterior } \\
\text { tentorial pit }\end{array}$ & $\begin{array}{l}\text { Anterior } \\
\text { tentorial pit }\end{array}$ \\
\hline- & - & - & $\begin{array}{l}\text { Apical } \\
\text { inflection of } \\
\text { clypeus }\end{array}$ & - & $\begin{array}{l}\text { Ventral ridge } \\
\text { of clypeus }\end{array}$ & $\begin{array}{l}\text { Ventral ridge } \\
\text { of clypeus** }\end{array}$ \\
\hline- & - & $\begin{array}{l}\text { Bulla (of } \\
\text { labrum)* }\end{array}$ & - & - & - & $\begin{array}{l}\text { Labral } \\
\text { bullae** }\end{array}$ \\
\hline- & - & $\begin{array}{l}\text { Circumocular } \\
\text { ridge }\end{array}$ & - & - & $\begin{array}{l}\text { Circumocular } \\
\text { ridge }\end{array}$ & $\begin{array}{l}\text { Circumocular } \\
\text { ridge }\end{array}$ \\
\hline- & - & - & $\begin{array}{l}\text { Condyle of } \\
\text { anterior } \\
\text { mandibular } \\
\text { articulation }\end{array}$ & - & $\begin{array}{l}\text { Pleurostomal } \\
\text { condyle }\end{array}$ & $\begin{array}{l}\text { Pleurostomal } \\
\text { condyle** }\end{array}$ \\
\hline- & - & $\begin{array}{l}\text { Dorso-lateral } \\
\text { angle of } \\
\text { clypeus }\end{array}$ & - & - & - & $\begin{array}{l}\text { Dorso-lateral } \\
\text { angle of } \\
\text { clypeus }\end{array}$ \\
\hline- & $\begin{array}{l}\text { Ridge of } \\
\text { epistomal } \\
\text { sulcus* }\end{array}$ & $\begin{array}{l}\text { Internal } \\
\text { manifestation } \\
\text { of epistomal } \\
\text { suture* }\end{array}$ & $\begin{array}{l}\text { Epistomal } \\
\text { ridge }\end{array}$ & - & $\begin{array}{l}\text { Epistomal } \\
\text { ridge }\end{array}$ & $\begin{array}{l}\text { Epistomal } \\
\text { ridge }\end{array}$ \\
\hline
\end{tabular}


Table 2. Continuation.

\begin{tabular}{|c|c|c|c|c|c|c|}
\hline RES'10 & RES'42 & CDM & RA\&M & $\mathbf{L V}$ & HAO & This Work \\
\hline- & Dorsal tentorial & - & - & - & Dorsal tentorial & $\begin{array}{l}\text { Median } \\
\text { expansion of } \\
\text { epistomal } \\
\text { ridge** }\end{array}$ \\
\hline- & $\begin{array}{l}\text { Dorsal tentorial } \\
\text { arm }\end{array}$ & - & - & - & $\begin{array}{l}\text { Dorsal tentorial } \\
\text { arm }\end{array}$ & $\begin{array}{l}\text { Dorsal tentorial } \\
\text { arm }\end{array}$ \\
\hline $\begin{array}{l}\text { Clypeal } \\
\text { suture }\end{array}$ & $\begin{array}{l}\text { Epistomal } \\
\text { sulcus }\end{array}$ & $\begin{array}{l}\text { Epistomal } \\
\text { suture }\end{array}$ & $\begin{array}{l}\text { Epistomal } \\
\text { suture }\end{array}$ & $\begin{array}{l}\text { Epistomal } \\
\text { sulcus }\end{array}$ & $\begin{array}{l}\text { Epistomal } \\
\text { sulcus }\end{array}$ & $\begin{array}{l}\text { Epistomal } \\
\text { sulcus** }\end{array}$ \\
\hline- & - & - & $\begin{array}{l}\text { Eutentorial } \\
\text { arm } \\
\text { (=part of } \\
\text { tentorial } \\
\text { arm?) }\end{array}$ & - & - & $\begin{array}{l}\text { Eutentorial arm } \\
\text { (=thickening of } \\
\text { tentorial arm)** }\end{array}$ \\
\hline- & - & $\begin{array}{l}\text { Fan-shaped } \\
\text { sheet } \\
\text { (=part of } \\
\text { anterior root)* }\end{array}$ & $\begin{array}{l}\text { Dorsal sheet } \\
\text { of anterior } \\
\text { tentorial arm }\end{array}$ & - & - & $\begin{array}{l}\text { Dorsal sheet of } \\
\text { anterior } \\
\text { tentorial arm }\end{array}$ \\
\hline $\begin{array}{l}\text { Foramen } \\
\text { magnum }\end{array}$ & $\begin{array}{l}\text { Foramen } \\
\text { magnum }\end{array}$ & $\begin{array}{l}\text { Foramen } \\
\text { magnum }\end{array}$ & $\begin{array}{l}\text { Foramen } \\
\text { magnum }\end{array}$ & $\begin{array}{l}\text { Occipital } \\
\text { foramen }\end{array}$ & $\begin{array}{l}\text { Occipital } \\
\text { foramen }\end{array}$ & $\begin{array}{l}\text { Occipital } \\
\text { foramen** }\end{array}$ \\
\hline - & 0 & $\begin{array}{l}\text { Funnel-shaped } \\
\text { sheet } \\
\text { (=part of } \\
\text { posterior root)* }\end{array}$ & $\begin{array}{l}\text { Fan-shaped } \\
\text { posterior } \\
\text { sheet of } \\
\text { tentorium }\end{array}$ & - & - & $\begin{array}{l}\text { Fan-shaped } \\
\text { sheet of } \\
\text { posterior } \\
\text { tentorial arm** }\end{array}$ \\
\hline $\begin{array}{l}\text { Thin vertical } \\
\text { plates* }\end{array}$ & Hypostoma* & Hypostoma & Hypostoma & - & Hypostoma & Hypostoma \\
\hline 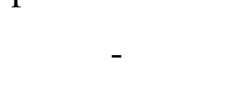 & $\begin{array}{l}\text { Hypostomal } \\
\text { bridge }\end{array}$ & $\begin{array}{l}\text { Hypostomal } \\
\text { bridge }\end{array}$ & 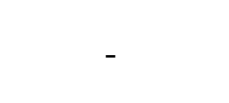 & - & $\begin{array}{l}\text { Hypostomal } \\
\text { bridge }\end{array}$ & $\begin{array}{l}\text { Hypostomal } \\
\text { bridge }\end{array}$ \\
\hline- & - & $\begin{array}{l}\text { Inner } \\
\text { subantennal } \\
\text { suture }\end{array}$ & $\begin{array}{l}\text { Subantennal } \\
\text { suture (inner } \\
\text { branch) }\end{array}$ & - & $\begin{array}{l}\text { Subantennal } \\
\text { groove }\end{array}$ & $\begin{array}{l}\text { Inner } \\
\text { subantennal } \\
\text { groove** }\end{array}$ \\
\hline- & - & $\begin{array}{l}\text { Lateral angle } \\
\text { of clypeus }\end{array}$ & 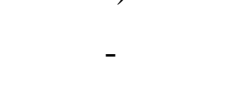 & - & - & $\begin{array}{l}\text { Lateral angle of } \\
\text { clypeus }\end{array}$ \\
\hline Gena & Gena* & Malar area & - & - & Malar space & Malar space** \\
\hline- & $\cos ^{2}$ & $\begin{array}{l}\text { Marginal area } \\
\text { of clypeus }\end{array}$ & - & - & $\begin{array}{l}\text { Ventral rim of } \\
\text { clypeus }\end{array}$ & $\begin{array}{l}\text { Ventral rim of } \\
\text { clypeus** }\end{array}$ \\
\hline $\begin{array}{l}\text { Maxillary } \\
\text { suspensorium }\end{array}$ & $\begin{array}{l}\text { Cranial } \\
\text { articulation of } \\
\text { maxillary cardo }\end{array}$ & $\begin{array}{l}\text { Maxillary } \\
\text { process of } \\
\text { hypostoma }\end{array}$ & - & - & $\begin{array}{l}\text { Maxillary } \\
\text { process of } \\
\text { hypostoma }\end{array}$ & $\begin{array}{l}\text { Maxillary } \\
\text { process of } \\
\text { hypostoma }\end{array}$ \\
\hline- & 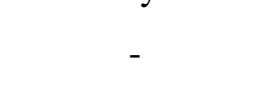 & $\begin{array}{l}\text { Occipital } \\
\text { sulcus }\end{array}$ & $\begin{array}{l}\text { Occipital } \\
\text { sulcus }\end{array}$ & - & S & $\begin{array}{l}\text { Arched sulcus } \\
\text { of occiput** }\end{array}$ \\
\hline $\begin{array}{l}\text { Occipital } \\
\text { region? }\end{array}$ & Occiput* & Occiput & 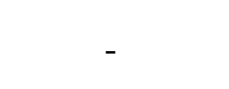 & - & Occiput & Occiput \\
\hline (2) & - & $\begin{array}{l}\text { Outer } \\
\text { subantennal } \\
\text { suture }\end{array}$ & $\begin{array}{l}\text { Subantennal } \\
\text { suture (outer } \\
\text { branch) }\end{array}$ & - & $\begin{array}{l}\text { Subantennal } \\
\text { groove }\end{array}$ & $\begin{array}{l}\text { Outer } \\
\text { subantennal } \\
\text { groove** }\end{array}$ \\
\hline
\end{tabular}


Table 2. Continuation.

\begin{tabular}{|c|c|c|c|c|c|c|}
\hline RES'10 & RES'42 & CDM & RA\&M & $\mathbf{L V}$ & HAO & This Work \\
\hline- & - & $\begin{array}{l}\text { Paramandibular } \\
\text { carina }\end{array}$ & - & - & - & $\begin{array}{l}\text { Paramandibular } \\
\text { carina }\end{array}$ \\
\hline- & - & $\begin{array}{l}\text { Paramandibular } \\
\text { process of } \\
\text { hypostoma }\end{array}$ & - & - & $\begin{array}{l}\text { Paramandibular } \\
\text { process of } \\
\text { hypostoma }\end{array}$ & $\begin{array}{l}\text { Paramandibular } \\
\text { process of } \\
\text { hypostoma }\end{array}$ \\
\hline- & - & $\begin{array}{l}\text { Paraocular } \\
\text { carina }\end{array}$ & $\begin{array}{l}\text { Paraocular } \\
\text { carina }\end{array}$ & - & $\begin{array}{l}\text { Paraocular } \\
\text { carina }\end{array}$ & $\begin{array}{l}\text { Paraocular } \\
\text { carina }\end{array}$ \\
\hline $\begin{array}{l}\text { Pharyngeal } \\
\text { plate }\end{array}$ & Oral plate & $\begin{array}{l}\text { Pharyngeal } \\
\text { plate }\end{array}$ & - & - & Sitophore & Sitophore** \\
\hline 1 & $\begin{array}{l}\text { Opening of } \\
\text { hypopharyngeal } \\
\text { glands }\end{array}$ & 1 & - & - & - & $\begin{array}{l}\text { Opening of } \\
\text { hypopharyngeal } \\
\text { glands }\end{array}$ \\
\hline- & Pharyngeal arm & Pharyngeal rod & - & - & - & Pharyngeal rod \\
\hline- & $\begin{array}{l}\text { Triangular fold } \\
\text { (=suboral fold, } \\
\text { suboral bib, } \\
\text { bib) }\end{array}$ & 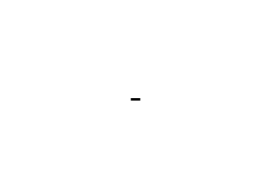 & - & - & $\begin{array}{l}\text { Hypopharyngeal } \\
\text { lobe }\end{array}$ & $\begin{array}{l}\text { Hypopharyngeal } \\
\text { lobe** }\end{array}$ \\
\hline- & 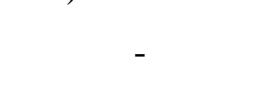 & - & - & - & $\begin{array}{l}\text { Posterior } \\
\text { tentorial arm }\end{array}$ & $\begin{array}{l}\text { Posterior } \\
\text { tentorial arm }\end{array}$ \\
\hline- & - & $\begin{array}{l}\text { Posterior root } \\
\text { (of tentorium)* } \\
\text { (=connection } \\
\text { of posterior } \\
\text { arm to head } \\
\text { wall) }\end{array}$ & - & - & 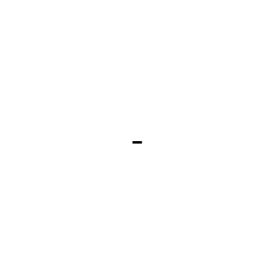 & $\begin{array}{l}\text { Posterior root of } \\
\text { tentorium** }\end{array}$ \\
\hline $\begin{array}{l}\text { Larger pit (c, } \\
\text { Fig. 9) }\end{array}$ & $\begin{array}{l}\text { Posterior } \\
\text { tentorial pit }\end{array}$ & $\begin{array}{l}\text { Posterior } \\
\text { tentorial pit }\end{array}$ & - & $\begin{array}{l}\text { Posterior } \\
\text { tentorial } \\
\text { pit }\end{array}$ & $\begin{array}{l}\text { Posterior } \\
\text { tentorial pit }\end{array}$ & $\begin{array}{l}\text { Posterior } \\
\text { tentorial pit }\end{array}$ \\
\hline- & $\begin{array}{l}\text { Postgenal } \\
\text { bridge }\end{array}$ & $\begin{array}{l}\text { Postgenal } \\
\text { bridge }\end{array}$ & - & $\begin{array}{l}\text { Postgenal } \\
\text { bridge }\end{array}$ & $\begin{array}{l}\text { Postgenal } \\
\text { bridge }\end{array}$ & $\begin{array}{l}\text { Postgenal } \\
\text { bridge }\end{array}$ \\
\hline- & - & $\begin{array}{l}\text { Postoccipital } \\
\text { bridge }\end{array}$ & 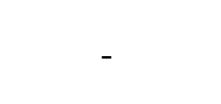 & - & 8 & $\begin{array}{l}\text { Postoccipital } \\
\text { bridge }\end{array}$ \\
\hline- & - & - & $\begin{array}{l}\text { Postoccipital } \\
\text { pouch }\end{array}$ & - & 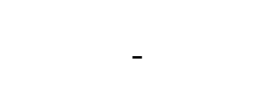 & $\begin{array}{l}\text { Postoccipital } \\
\text { pouch }\end{array}$ \\
\hline- & - & $\begin{array}{l}\text { Postoccipital } \\
\text { suture }\end{array}$ & - & - & $\begin{array}{l}\text { Postoccipital } \\
\text { suture }\end{array}$ & $\begin{array}{l}\text { Postoccipital } \\
\text { suture }\end{array}$ \\
\hline $\begin{array}{l}\text { Occipital } \\
\text { region? }\end{array}$ & Postocciput* & Postocciput & - & - & Postocciput & Postocciput \\
\hline $\begin{array}{l}\text { Occipital } \\
\text { region }\end{array}$ & - & - & - & - & Occipital arch & Occipital $\operatorname{arch}^{* *}$ \\
\hline ic givin & - & $\begin{array}{l}\text { Preoccipital } \\
\text { ridge }\end{array}$ & - & $\begin{array}{l}\text { Occipital } \\
\text { carina }\end{array}$ & Occipital carina & $\begin{array}{l}\text { Occipital } \\
\text { carina** }\end{array}$ \\
\hline $\begin{array}{l}\text { Small rod } \\
\text { (ten, Fig. 9) }\end{array}$ & $\begin{array}{l}\text { Tentorial } \\
\text { bridge }\end{array}$ & $\begin{array}{l}\text { Primary } \\
\text { tentorial bridge }\end{array}$ & - & - & Tentorial bridge & $\begin{array}{l}\text { Tentorial } \\
\text { bridge** }\end{array}$ \\
\hline
\end{tabular}


Table 2. Continuation.

\begin{tabular}{|c|c|c|c|c|c|c|}
\hline RES'10 & RES' 42 & CDM & RA\&M & $\mathbf{L V}$ & HAO & This Work \\
\hline $\begin{array}{l}\text { Fossa of the } \\
\text { proboscis }\end{array}$ & $\begin{array}{l}\text { Fossa of the } \\
\text { proboscis }\end{array}$ & $\begin{array}{l}\text { Proboscidial } \\
\text { fossa }\end{array}$ & $\begin{array}{l}\text { Proboscidial } \\
\text { fossa }\end{array}$ & - & $\begin{array}{l}\text { Proboscidial } \\
\text { fossa }\end{array}$ & $\begin{array}{l}\text { Proboscidial } \\
\text { fossa }\end{array}$ \\
\hline- & $\begin{array}{l}\text { Shelflike plate } \\
\text { (a, Fig. I) }\end{array}$ & $\begin{array}{l}\text { Secondary } \\
\text { tentorial bridge }\end{array}$ & $\begin{array}{l}\text { Secondary } \\
\text { tentorial } \\
\text { bridge }\end{array}$ & - & 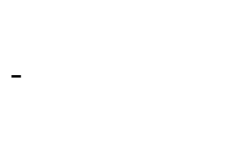 & $\begin{array}{l}\text { Secondary } \\
\text { tentorial bridge }\end{array}$ \\
\hline- & - & Subantennal pit & - & - & - & Subantennal pit \\
\hline Tentorium & Tentorium* & Tentorium & Tentorium & - & Tentorium & Tentorium \\
\hline- & $\begin{array}{l}\text { Tentorial bar } \\
\text { (=tentorium?) }\end{array}$ & - & $\begin{array}{l}\text { Tentorial } \\
\text { arm } \\
\text { (=tentorium) }\end{array}$ & - & $\begin{array}{l}\text { Tentorial arm } \\
\text { (=tentorium) }\end{array}$ & $\begin{array}{l}\text { Tentorial arm } \\
\text { (=tentorium) }\end{array}$ \\
\hline- & - & - & $\begin{array}{l}\text { Thickening } \\
\text { of secondary } \\
\text { tentorial } \\
\text { bridge }\end{array}$ & - & - & $\begin{array}{l}\text { Thickening of } \\
\text { secondary } \\
\text { tentorial bridge }\end{array}$ \\
\hline- & - & - & $\begin{array}{l}\text { Lateral } \\
\text { expansion of } \\
\text { internal } \\
\text { thickening }\end{array}$ & - & - & $\begin{array}{l}\text { Lateral } \\
\text { expansion of the } \\
\text { thickening of } \\
\text { secondary } \\
\text { tentorial } \\
\text { bridge** }\end{array}$ \\
\hline- & $\begin{array}{l}\text { Subforaminal } \\
\text { bridge } \\
\text { (=postgenal, } \\
\text { hypostomal } \\
\text { bridges) }\end{array}$ & - & - & - & $\begin{array}{l}\text { Subforaminal } \\
\text { bridge }\end{array}$ & $\begin{array}{l}\text { Subforaminal } \\
\text { bridge }\end{array}$ \\
\hline
\end{tabular}


Table 3. Morphological terminology utilized by different authors to make reference to some mandible structures. ${ }^{*}=$ terms not explicitly proposed by the authors in those works, but used in morphological descriptions or figure legends; ${ }^{* *}=$ changes of terminology or new terms introduced to make correspondence to more general terms already used in other hymenopteran groups. Abbreviations: CDM, C. D. Michener; HAO, Hymenoptera Anatomy Ontology; M\&F, Michener \& Fraser; RES, R. E. Snodgrass; DSP\&al, this work.

\begin{tabular}{|c|c|c|c|c|c|}
\hline RES'10 & RES'42 & CDM & M\&F & HAO & This Work \\
\hline- & - & - & $\begin{array}{l}\text { Apex (=first } \\
\text { tooth of } \\
\text { rutellum) }\end{array}$ & $\begin{array}{l}\text { Mandibular } \\
\text { tooth }\end{array}$ & $\begin{array}{l}\text { Tooth of } \\
\text { rutellum } \\
\text { (=first tooth of } \\
\text { rutellum)** }\end{array}$ \\
\hline- & - & $\begin{array}{l}\text { Abductor } \\
\text { swelling of } \\
\text { mandible }\end{array}$ & $\begin{array}{l}\text { Abductor } \\
\text { swelling }\end{array}$ & - & $\begin{array}{l}\text { Abductor } \\
\text { swelling } \\
\text { (of mandible) }\end{array}$ \\
\hline- & - & - & $\begin{array}{l}\text { Acetabular } \\
\text { carina }\end{array}$ & - & $\begin{array}{l}\text { Acetabular } \\
\text { carina } \\
\text { (of mandible) }\end{array}$ \\
\hline- & - & - & $\begin{array}{l}\text { Acetabular } \\
\text { groove }\end{array}$ & $\begin{array}{l}\text { Acetabular } \\
\text { groove }\end{array}$ & $\begin{array}{l}\text { Acetabular } \\
\text { groove } \\
\text { (of mandible) }\end{array}$ \\
\hline- & - & $\begin{array}{l}\text { Adductor angle } \\
\text { of mandible }\end{array}$ & $\begin{array}{l}\text { Adductor } \\
\text { convexity }\end{array}$ & - & $\begin{array}{l}\text { Adductor } \\
\text { convexity } \\
\text { (of mandible) }\end{array}$ \\
\hline- & - & - & Adductor ridge & - & $\begin{array}{l}\text { Adductor ridge } \\
\text { (of mandible) }\end{array}$ \\
\hline $\begin{array}{l}\text { Apodeme of } \\
\text { extensor } \\
\text { muscle of } \\
\text { mandible* }\end{array}$ & $\begin{array}{l}\text { Abductor } \\
\text { apodeme* }\end{array}$ & $\begin{array}{l}\text { Apodeme of } \\
\text { abductor } \\
\text { muscle of } \\
\text { mandible }\end{array}$ & $\begin{array}{l}\text { Apodeme of } \\
\text { the abductor } \\
\text { muscle }\end{array}$ & - & $\begin{array}{l}\text { Mandibular } \\
\text { abductor } \\
\text { muscle } \\
\text { apodeme** }\end{array}$ \\
\hline $\begin{array}{l}\text { Apodeme of } \\
\text { flexor muscle } \\
\text { of mandible* }\end{array}$ & $\begin{array}{l}\text { Adductor } \\
\text { apodeme* }\end{array}$ & $\begin{array}{l}\text { Apodeme of } \\
\text { adductor } \\
\text { muscle of } \\
\text { mandible }\end{array}$ & $\begin{array}{l}\text { Apodeme of } \\
\text { the adductor } \\
\text { muscle }\end{array}$ & $\begin{array}{l}\text { Mandibular } \\
\text { adductor } \\
\text { muscle } \\
\text { apodeme }\end{array}$ & $\begin{array}{l}\text { Mandibular } \\
\text { adductor } \\
\text { muscle } \\
\text { apodeme** }\end{array}$ \\
\hline- & - & - & $\begin{array}{l}\text { Condylar } \\
\text { groove }\end{array}$ & $\begin{array}{l}\text { Condylar } \\
\text { groove }\end{array}$ & $\begin{array}{l}\text { Condylar } \\
\text { groove } \\
\text { (of mandible) }\end{array}$ \\
\hline- & - & - & Condylar ridge & $\begin{array}{l}\text { Condylar carina } \\
\text { of the mandible }\end{array}$ & $\begin{array}{l}\text { Condylar } \\
\text { carina } \\
\text { (of } \\
\text { mandible)** }\end{array}$ \\
\hline- & - & - & Fimbrial carina & - & $\begin{array}{l}\text { Fimbrial carina } \\
\text { (of mandible) }\end{array}$ \\
\hline- & - & - & Fimbriate line & - & Fimbriate line \\
\hline- & - & - & $\begin{array}{l}\text { Inner surface } \\
\text { (of mandible) }\end{array}$ & - & $\begin{array}{l}\text { Inner surface } \\
\text { (of mandible) }\end{array}$ \\
\hline- & - & $\begin{array}{l}\text { Mandibular } \\
\text { acetabulum }\end{array}$ & $\begin{array}{l}\text { Mandibular } \\
\text { acetabulum }\end{array}$ & $\begin{array}{l}\text { Mandibular } \\
\text { acetabulum }\end{array}$ & $\begin{array}{l}\text { Mandibular } \\
\text { acetabulum }\end{array}$ \\
\hline- & - & $\begin{array}{l}\text { Mandibular } \\
\text { condyle }\end{array}$ & $\begin{array}{l}\text { Mandibular } \\
\text { condyle }\end{array}$ & $\begin{array}{l}\text { Mandibular } \\
\text { condyle }\end{array}$ & $\begin{array}{l}\text { Mandibular } \\
\text { condyle }\end{array}$ \\
\hline- & - & - & Oblique groove & 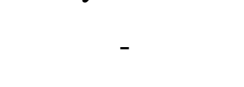 & $\begin{array}{l}\text { Oblique groove } \\
\text { (of mandible) }\end{array}$ \\
\hline
\end{tabular}


Table 3. Continuation.

\begin{tabular}{|c|c|c|c|c|c|}
\hline RES'10 & RES'42 & CDM & M\&F & HAO & This Work \\
\hline- & - & - & Outer groove & Outer groove & $\begin{array}{l}\text { Outer groove } \\
\text { (of mandible) }\end{array}$ \\
\hline- & - & - & Outer ridge & Outer ridge & $\begin{array}{l}\text { Outer ridge (of } \\
\text { mandible) }\end{array}$ \\
\hline- & - & - & $\begin{array}{l}\text { Outer surface } \\
\text { (of mandible) }\end{array}$ & $\begin{array}{l}\text { Lateral surface } \\
\text { (of mandible) }\end{array}$ & $\begin{array}{l}\text { Outer surface } \\
\text { (of mandible) }\end{array}$ \\
\hline- & - & - & Pollex & Pollex & Pollex \\
\hline- & - & Subapical tooth & $\begin{array}{l}\text { Preapical tooth } \\
\text { (=apex of } \\
\text { pollex, tooth of } \\
\text { pollex) }\end{array}$ & Preapical tooth & Preapical tooth \\
\hline- & - & - & Rutellum & - & Rutellum \\
\hline
\end{tabular}


Table 4. Matrix of character states distribution. - = inapplicable, ? = unknown/doubtful homology statement. Abbreviations: API, Apina; BOM, Bombina; CEN, Centridini; EUG, Euglossina; MEL, Meliponina.

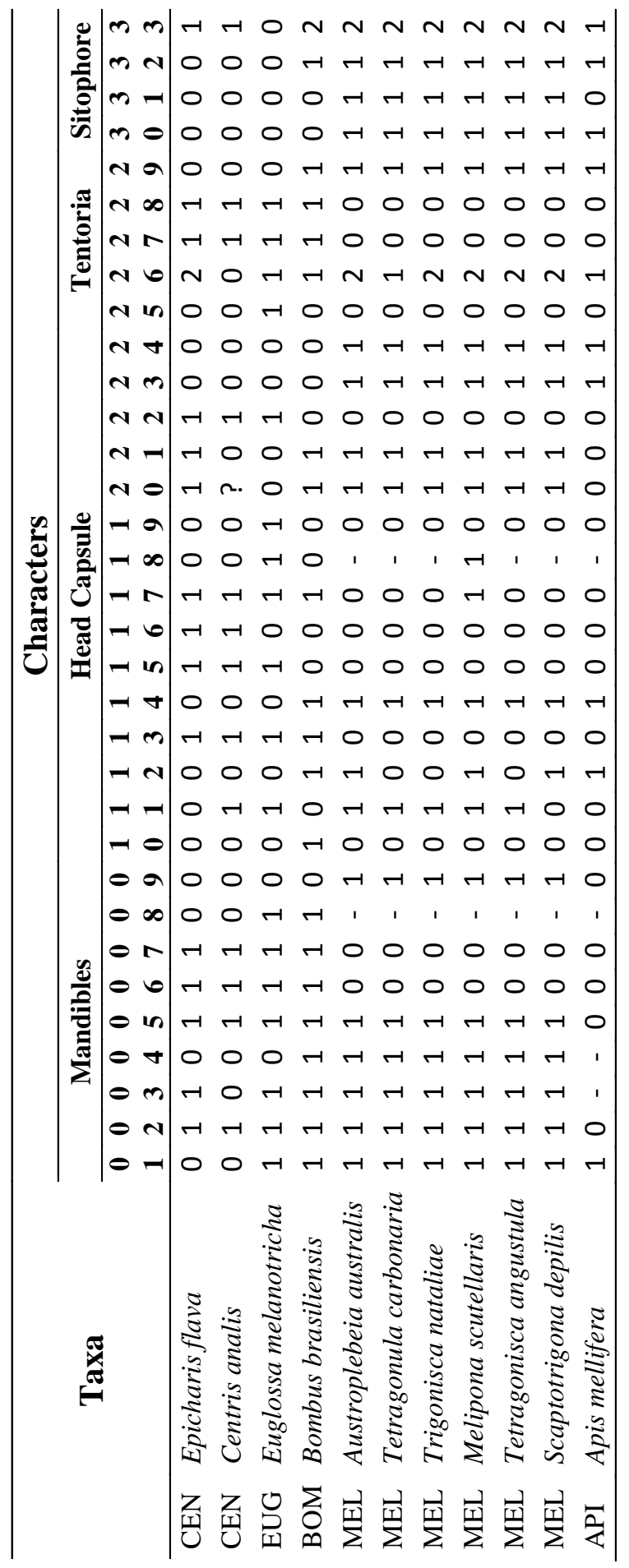



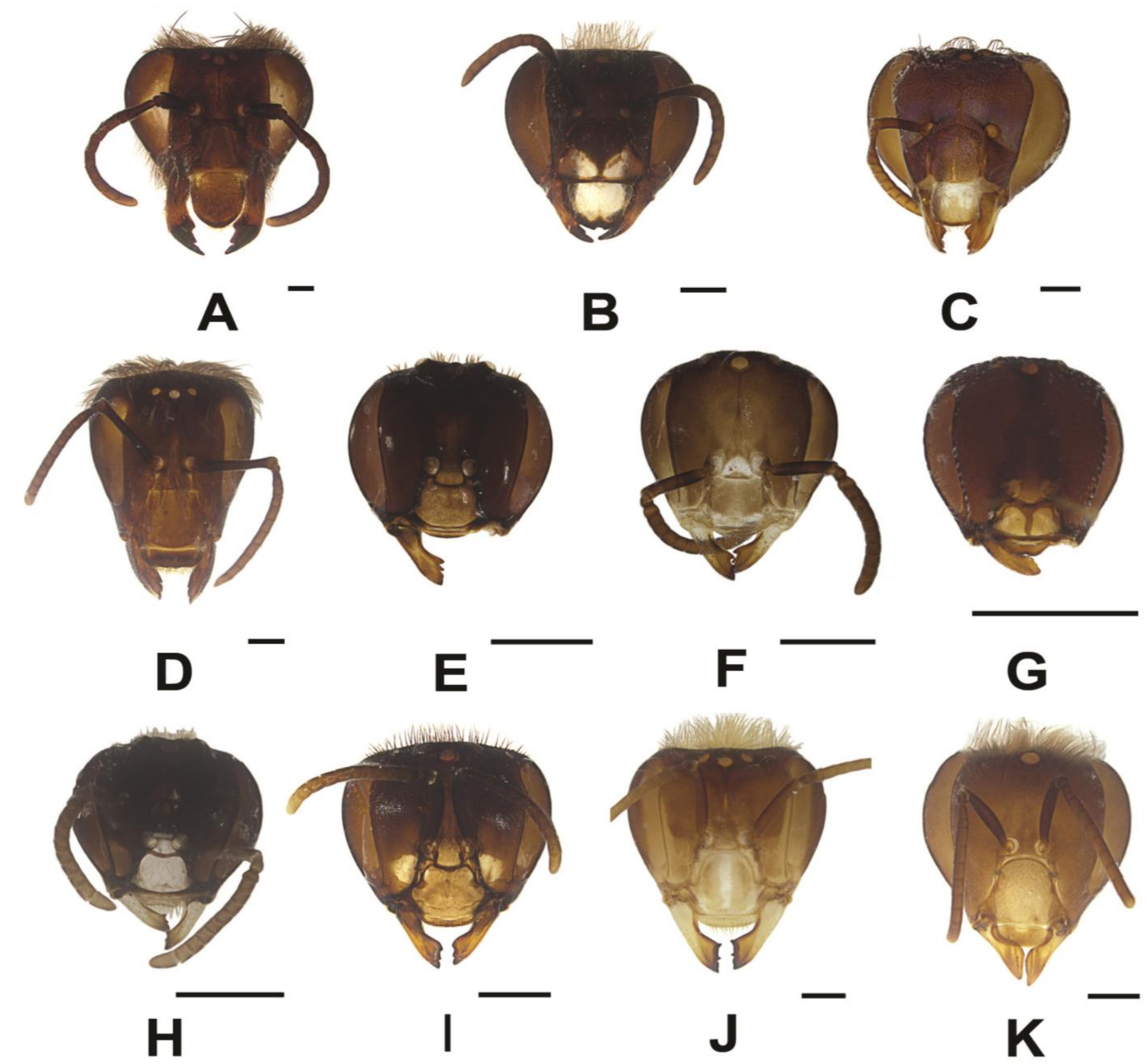

Figure 1. Images of heads in frontal view of cleared specimens. A. Epicharis flava Friese, 1900; B. Centris analis (Fabricius, 1804); C. Euglossa melanotricha Moure, 1967; D. Bombus brasiliensis Lepeletier, 1836; E. Tetragonula carbonaria (Smith, 1854); F. Austroplebeia australis (Friese, 1898); G. Trigonisca nataliae (Moure, 1950); H. Tetragonisca angustula (Latreille, 1811); I. Scaptotrigona depilis (Moure, 1942); J. Melipona scutellaris Latreille, 1811; K. Apis mellifera Linnaeus, 1758. Scale bar $=1 \mathrm{~mm}$. 

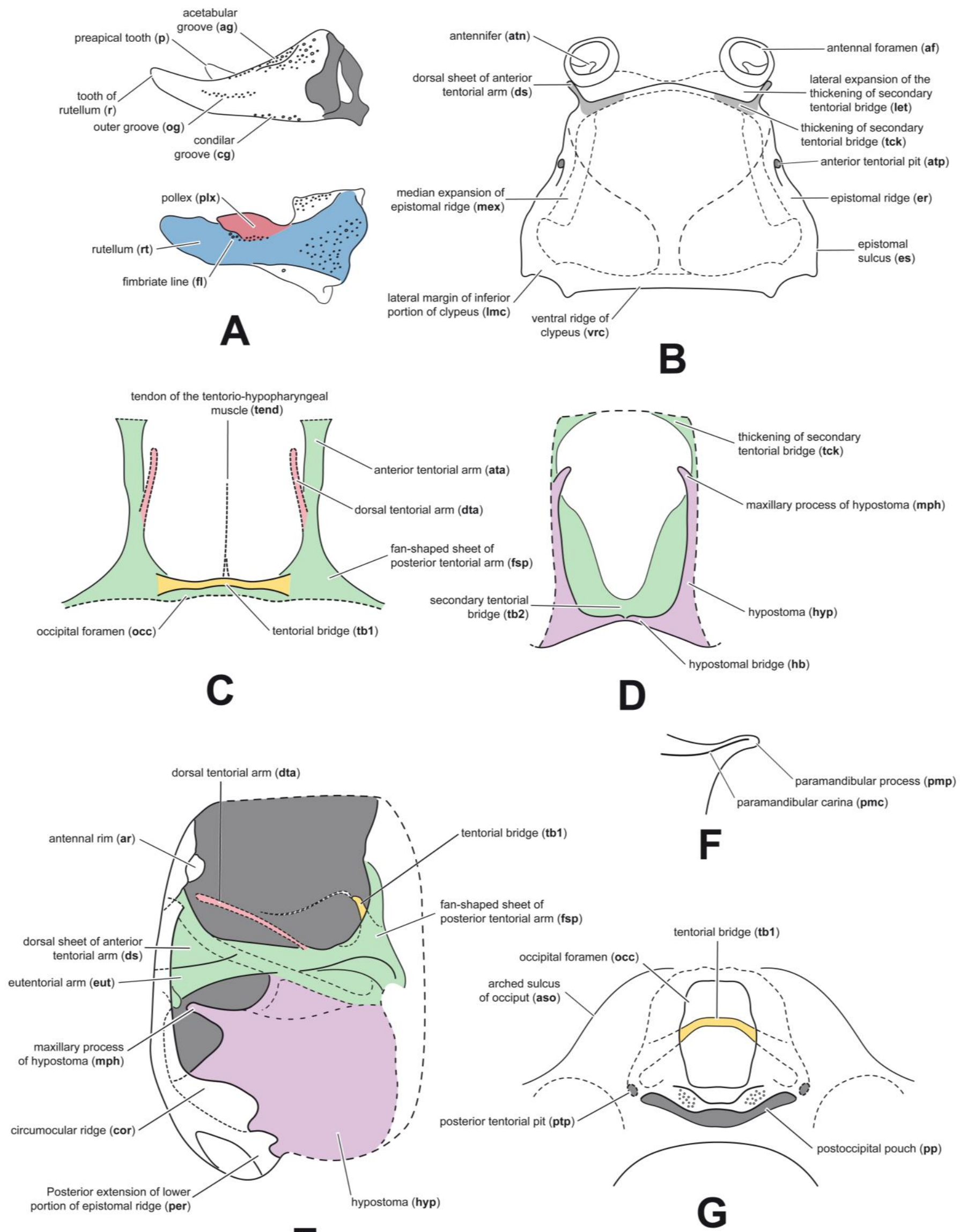

Figure 2. Explanatory figures based on structures pertaining to the head of Centris analis (Fabricius, 1804). A. Left mandible; top = external view, bottom = internal view; B. Epistomal ridge examined through transparency; frontal view; C. Tentoria; dorsal view; D. Hypostoma and secondary tentorial bridge; ventral view; E. Tentoria and hypostoma; lateral view; F. Paramandibular process of hypostoma, latero-ventral view; G. Occiput and postocciput; posterior view. 

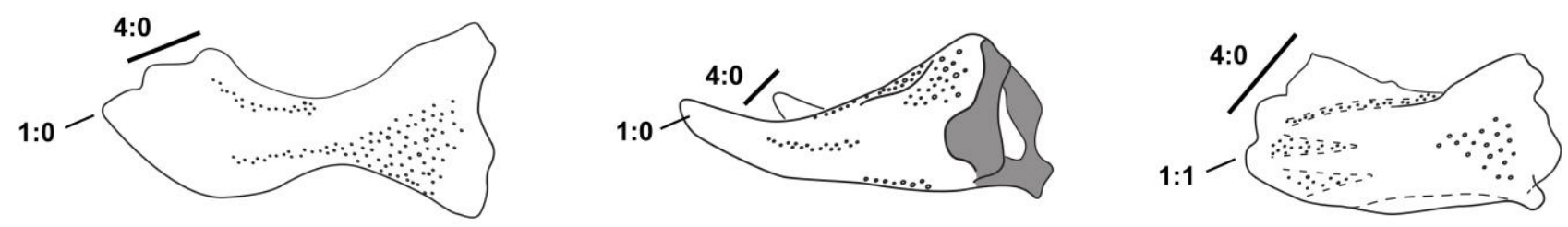

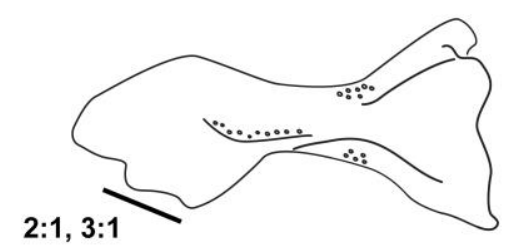

A

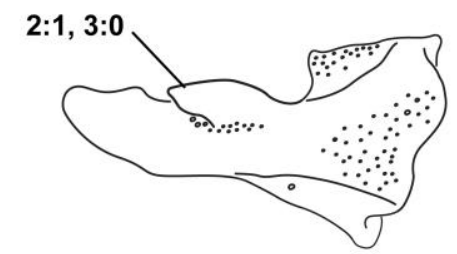

B

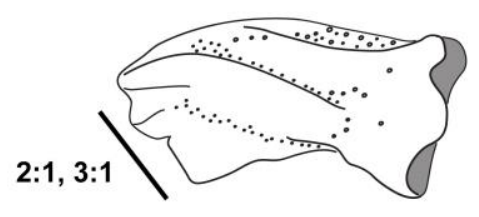

C
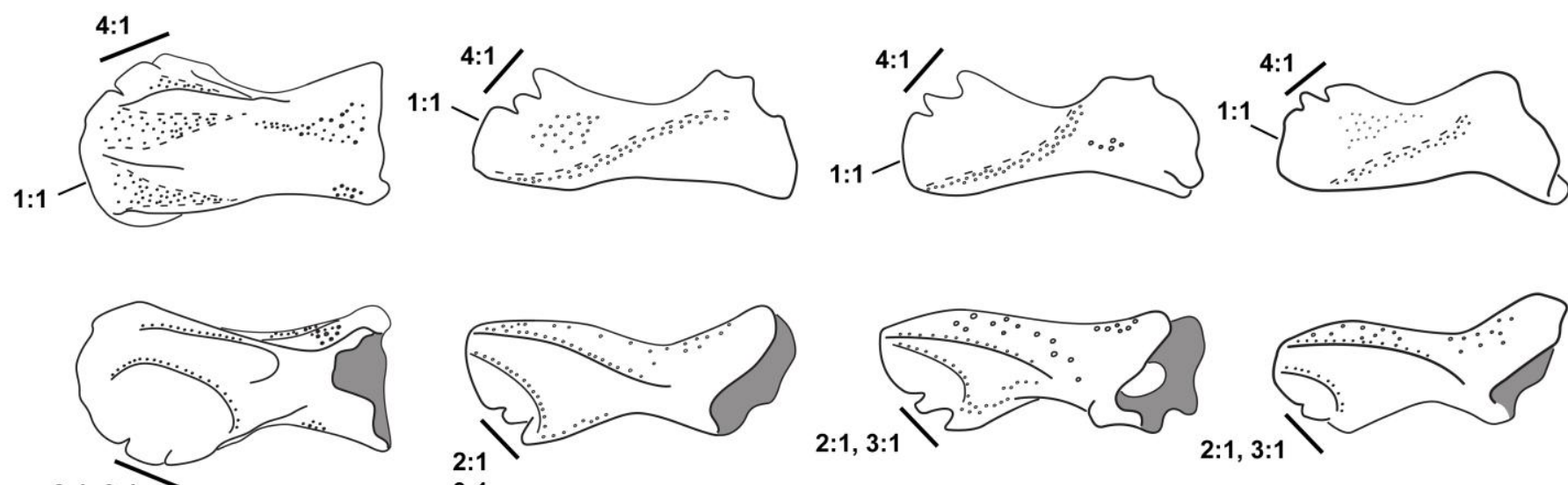

$2: 1,3: 1$

D

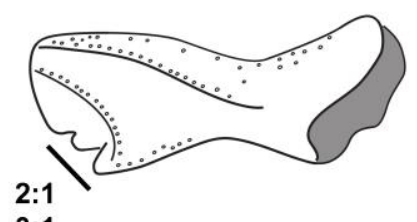

E
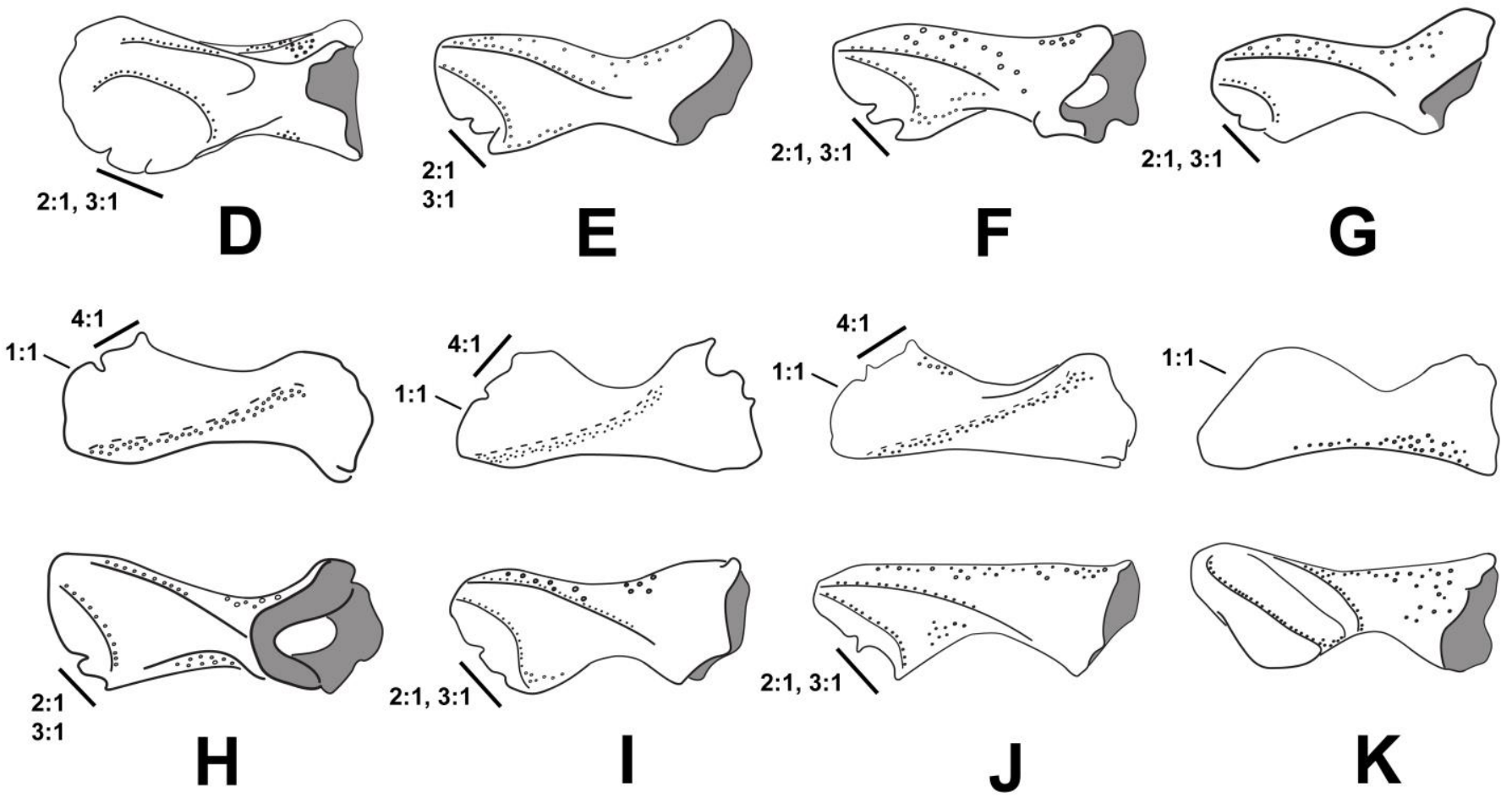

Figure 3. Left mandibles; top = external view, bottom = internal view; apex to the left. A. Epicharis flava Friese, 1900; B. Centris analis (Fabricius, 1804); C. Euglossa melanotricha Moure, 1967; D. Bombus brasiliensis Lepeletier, 1836; E. Tetragonula carbonaria (Smith, 1854); F. Austroplebeia australis (Friese, 1898); G. Trigonisca nataliae (Moure, 1950); H. Tetragonisca angustula (Latreille, 1811); I. Scaptotrigona depilis (Moure, 1942); J. Melipona scutellaris Latreille, 1811; K. Apis mellifera Linnaeus, 1758. 
Figure 4. (next page) Epistomal ridges; frontal view. A. Epicharis flava Friese, 1900; B. Centris analis (Fabricius, 1804); C. Euglossa melanotricha Moure, 1967; D. Bombus brasiliensis Lepeletier, 1836; E. Tetragonula carbonaria (Smith, 1854); F. Austroplebeia australis (Friese, 1898); G. Trigonisca nataliae (Moure, 1950); H. Tetragonisca angustula (Latreille, 1811); I. Scaptotrigona depilis (Moure, 1942); J. Melipona scutellaris Latreille, 1811; K. Apis mellifera Linnaeus, 1758. 

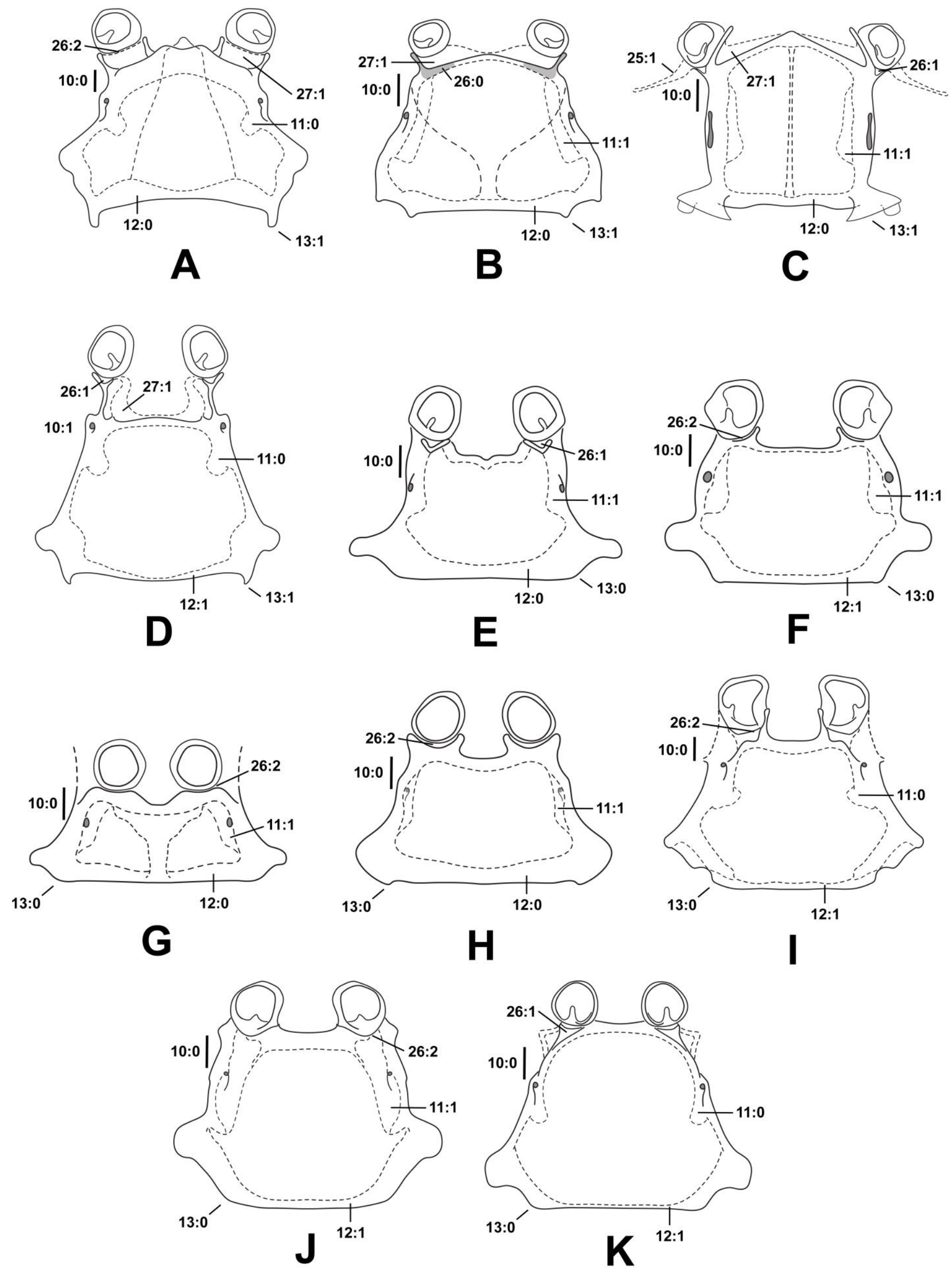
Figure 5. (next page) Occiput and postocciput; posterior view. A. Epicharis flava Friese, 1900; B. Centris analis (Fabricius, 1804); C. Euglossa melanotricha Moure, 1967; D. Bombus brasiliensis Lepeletier, 1836; E. Tetragonula carbonaria (Smith, 1854); F. Austroplebeia australis (Friese, 1898); G. Trigonisca nataliae (Moure, 1950); H. Tetragonisca angustula (Latreille, 1811); I. Scaptotrigona depilis (Moure, 1942); J. Melipona scutellaris Latreille, 1811; K. Apis mellifera Linnaeus, 1758. 


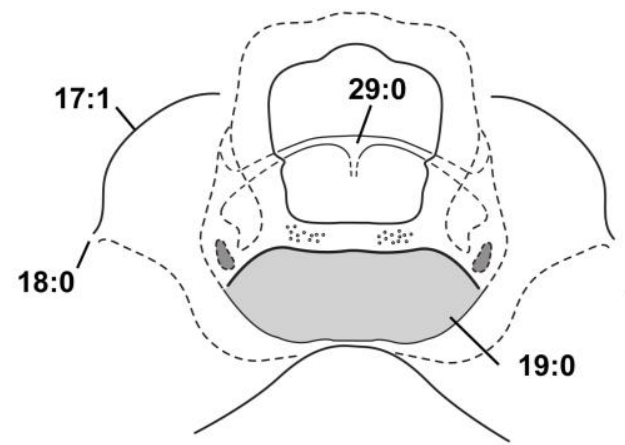

A

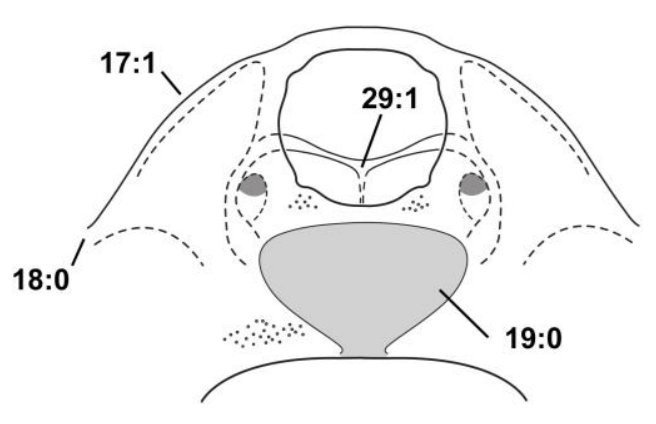

D

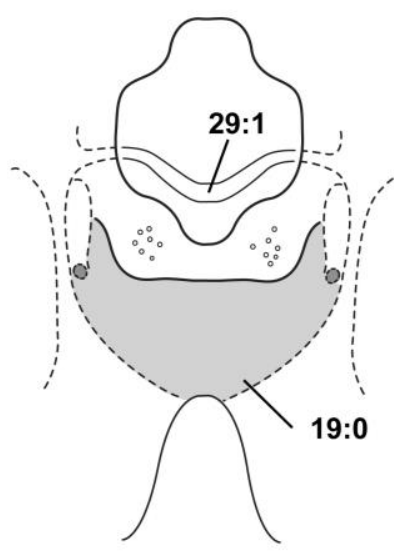

G

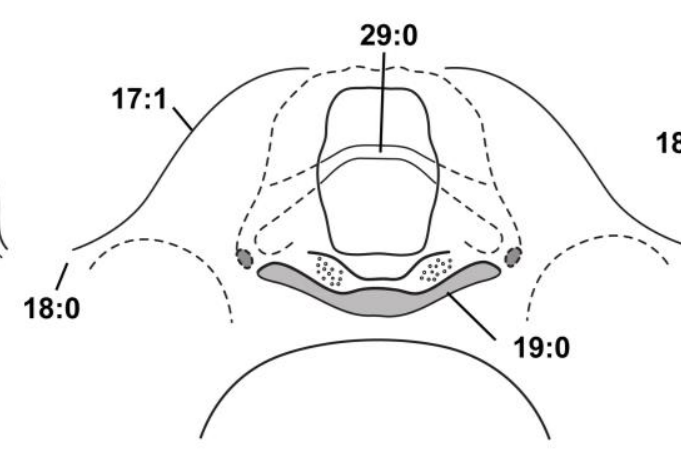

B

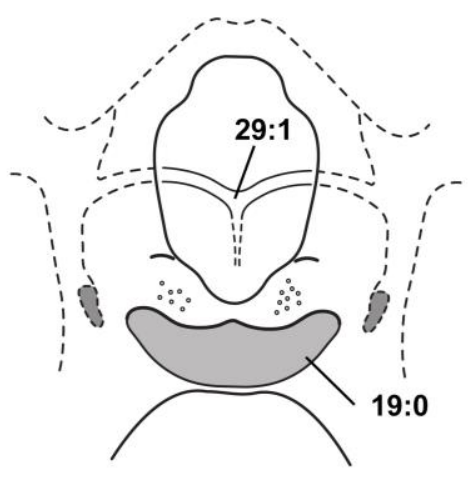

E

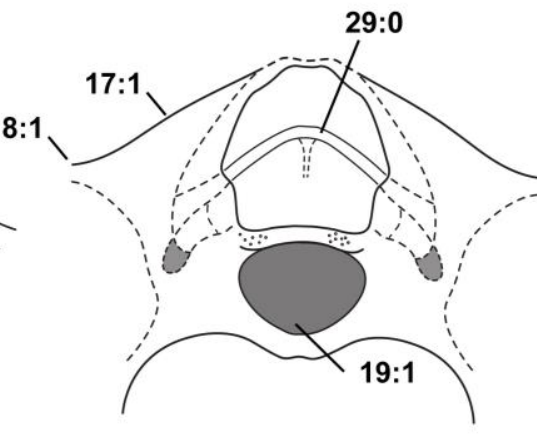

C

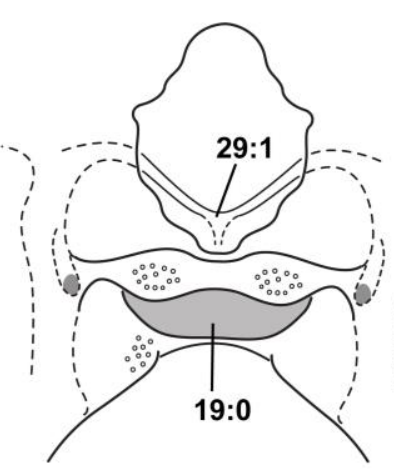

F

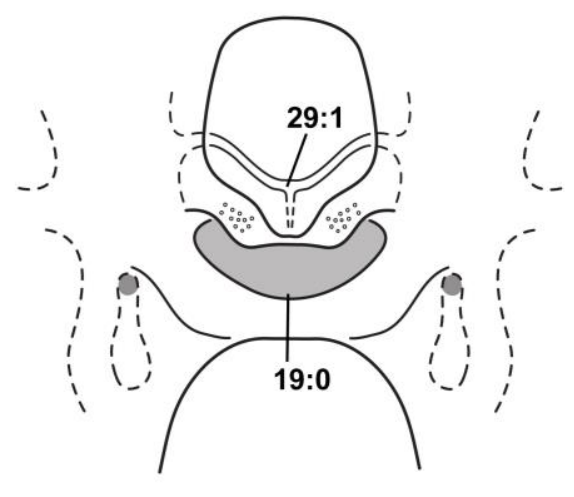

H
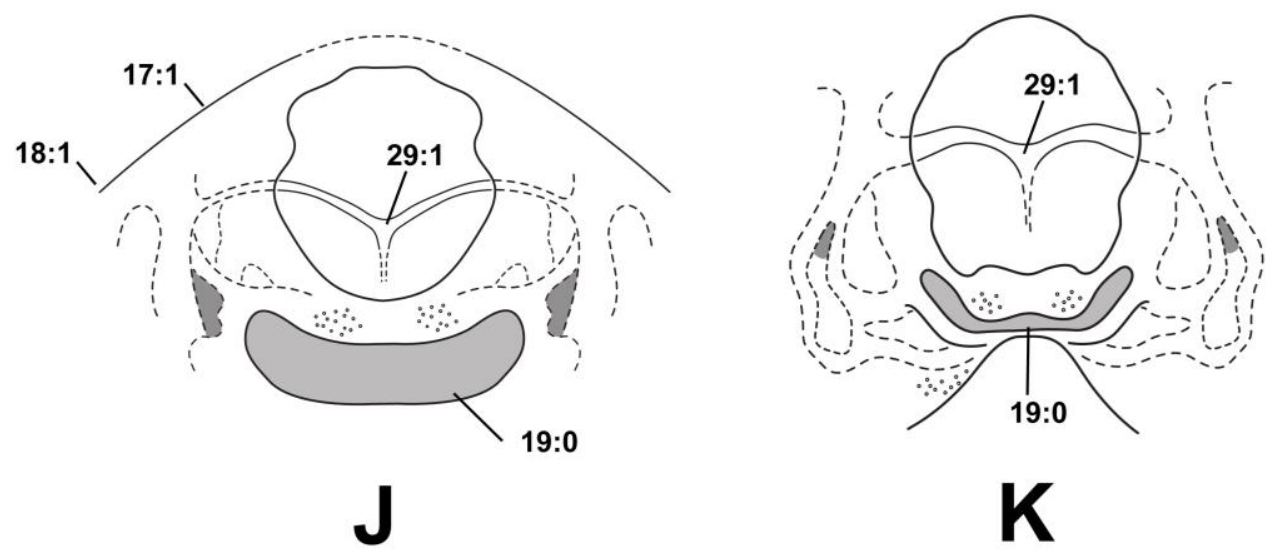
Figure 6. (next page) Hypostoma and secondary tentorial bridge; ventral view, anterior to the top. A. Epicharis flava Friese, 1900; B. Centris analis (Fabricius, 1804); C. Euglossa melanotricha Moure, 1967; D. Bombus brasiliensis Lepeletier, 1836; E. Tetragonula carbonaria (Smith, 1854); F. Austroplebeia australis (Friese, 1898); G. Trigonisca nataliae (Moure, 1950); H. Tetragonisca angustula (Latreille, 1811); I. Scaptotrigona depilis (Moure, 1942); J. Melipona scutellaris Latreille, 1811; K. Apis mellifera Linnaeus, 1758. 


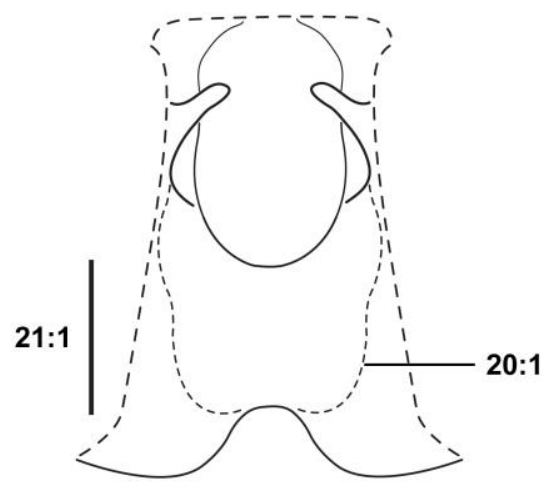

A

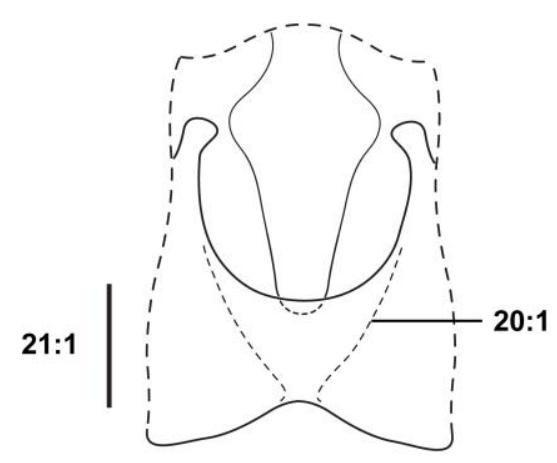

D

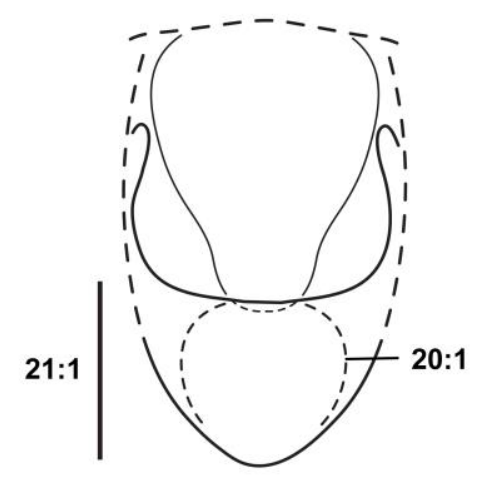

G

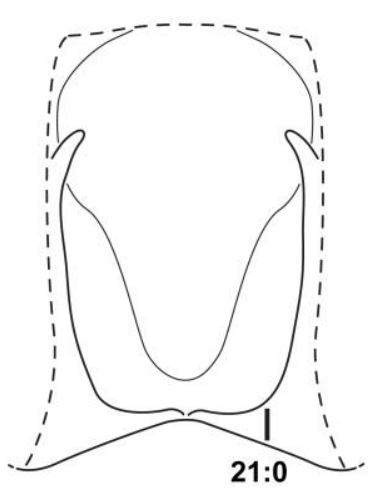

B

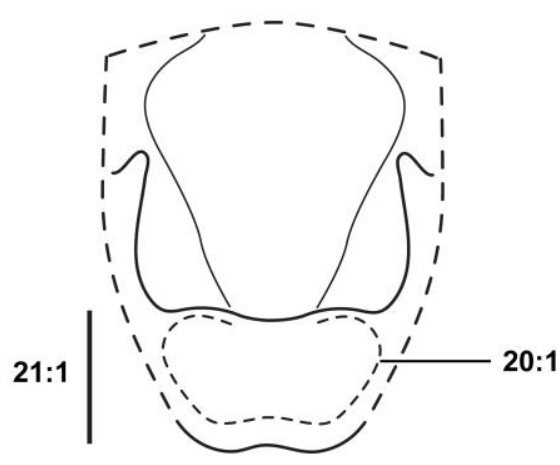

E

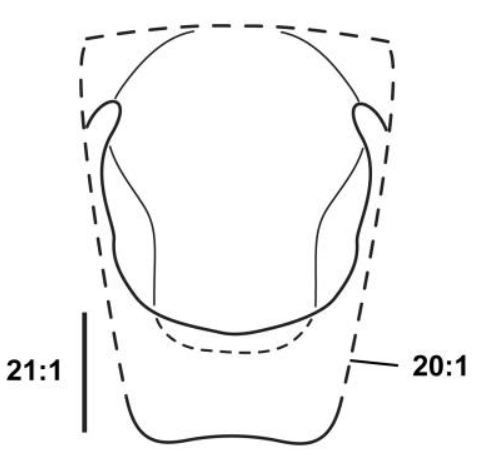

H

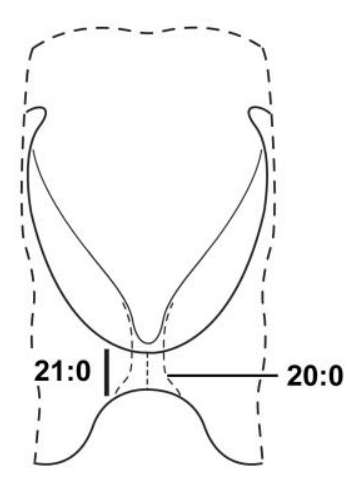

C

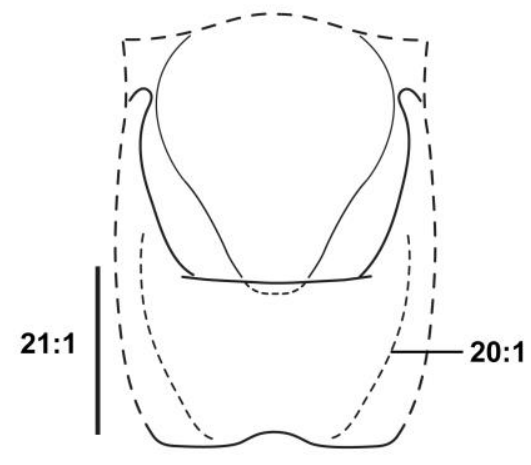

F

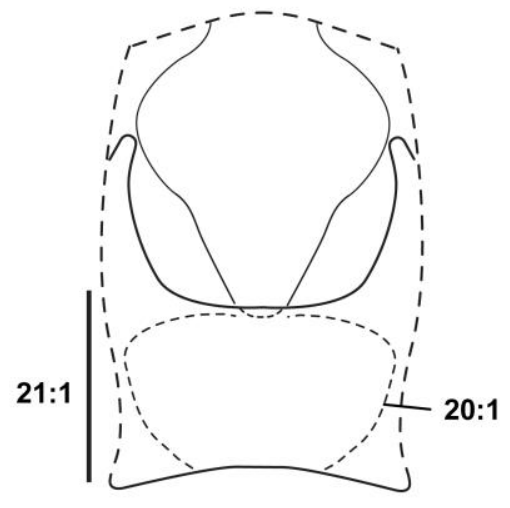

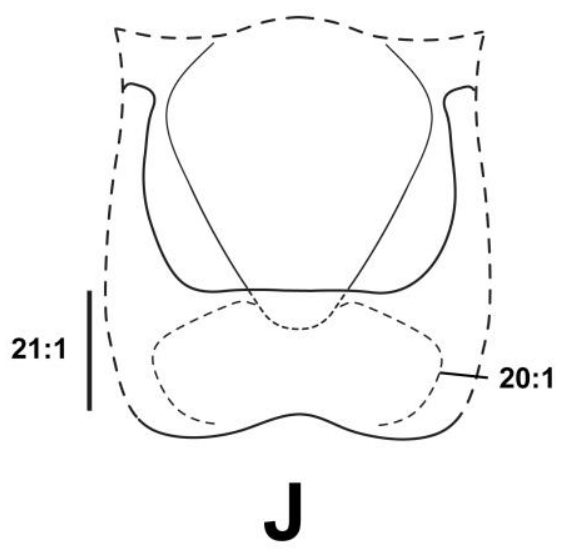

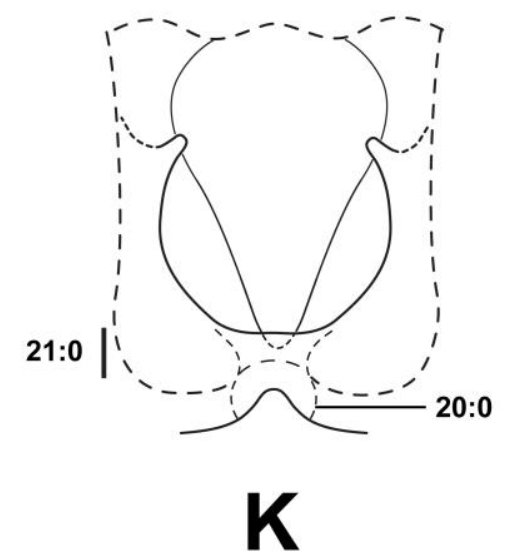




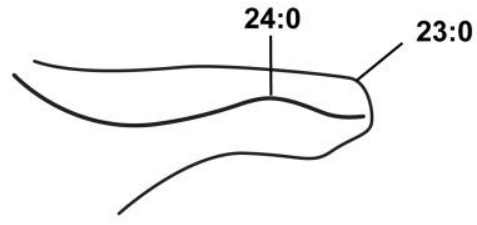

A

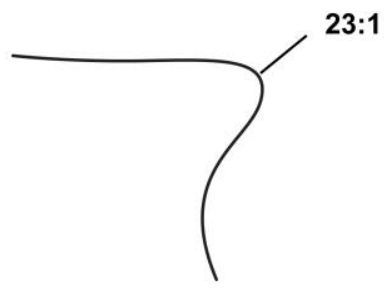

E

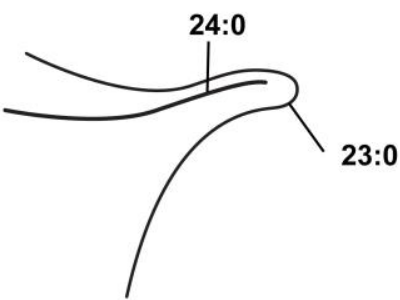

B

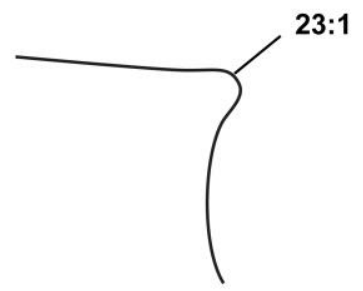

F

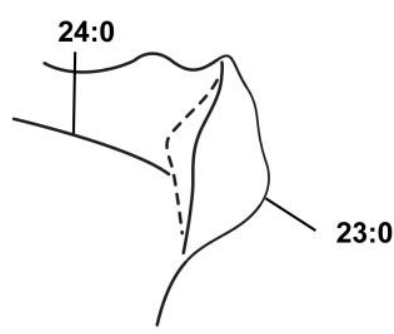

C

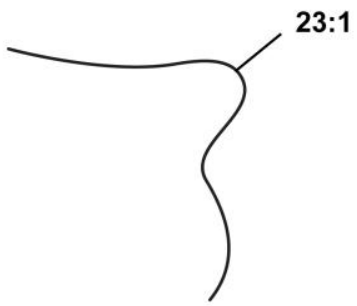

G

23:1

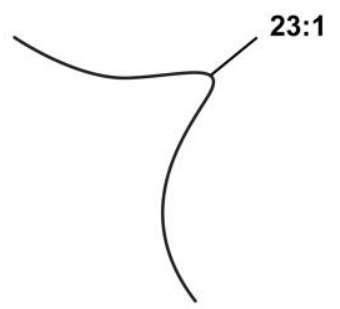

J

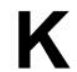

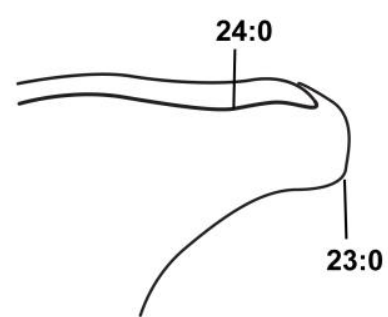

D

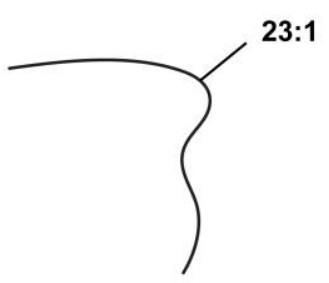

H

23:1

Figure 7. Paramandibular process of hypostoma; lateroventral view, anterior to the right. A. Epicharis flava Friese, 1900; B. Centris analis (Fabricius, 1804); C. Euglossa melanotricha Moure, 1967; D. Bombus brasiliensis Lepeletier, 1836; E. Tetragonula carbonaria (Smith, 1854); F. Austroplebeia australis (Friese, 1898); G. Trigonisca nataliae (Moure, 1950); H. Tetragonisca angustula (Latreille, 1811); I. Scaptotrigona depilis (Moure, 1942); J. Melipona scutellaris Latreille, 1811; K. Apis mellifera Linnaeus, 1758. 
Figure 8. (next page) Tentorium and hypostoma; lateral view, anterior to the left. A. Epicharis flava Friese, 1900; B. Centris analis (Fabricius, 1804); C. Euglossa melanotricha Moure, 1967; D. Bombus brasiliensis Lepeletier, 1836; E. Tetragonula carbonaria (Smith, 1854); F. Austroplebeia australis (Friese, 1898); G. Trigonisca nataliae (Moure, 1950); H. Tetragonisca angustula (Latreille, 1811); I. Scaptotrigona depilis (Moure, 1942); J. Melipona scutellaris Latreille, 1811; K. Apis mellifera Linnaeus, 1758. 


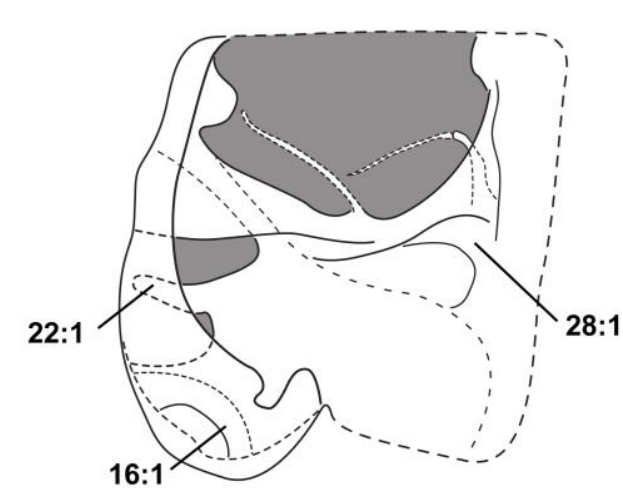

A

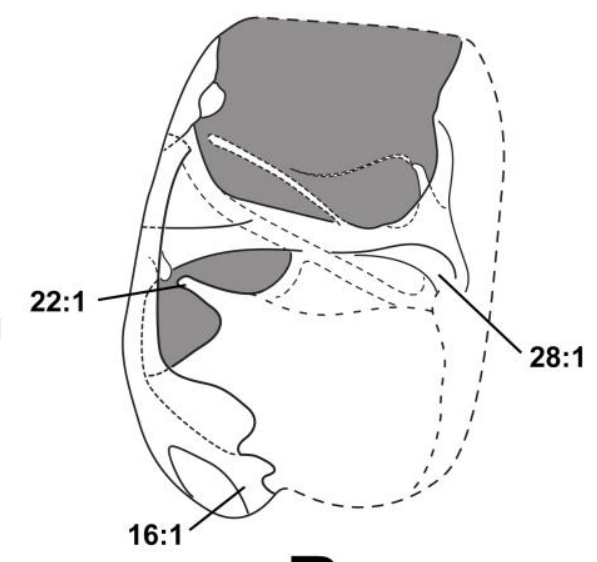

B

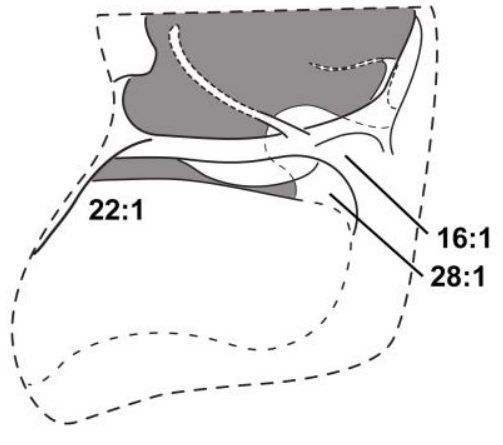

C

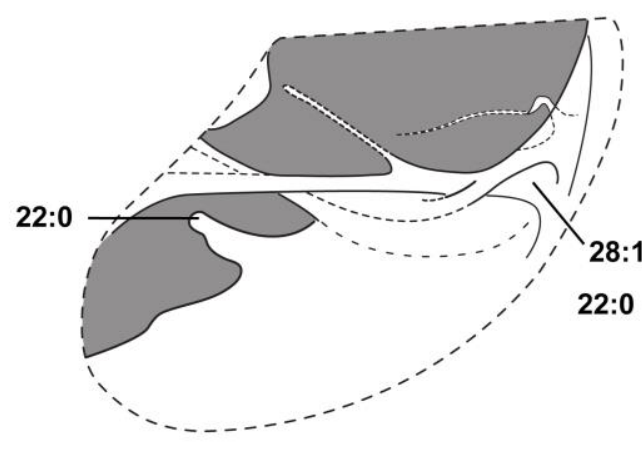

D

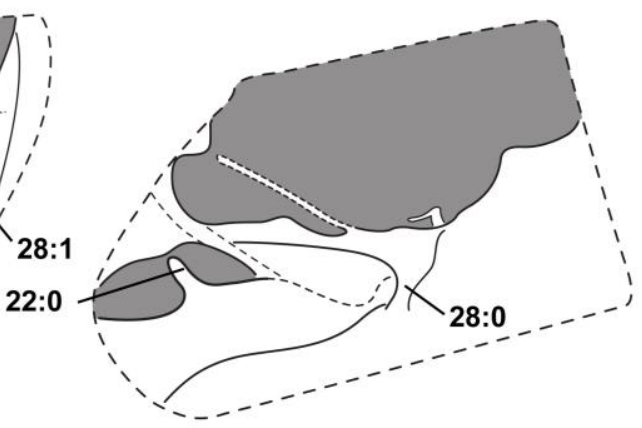

E

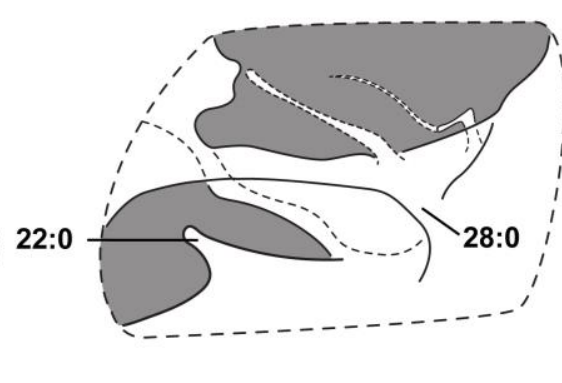

F
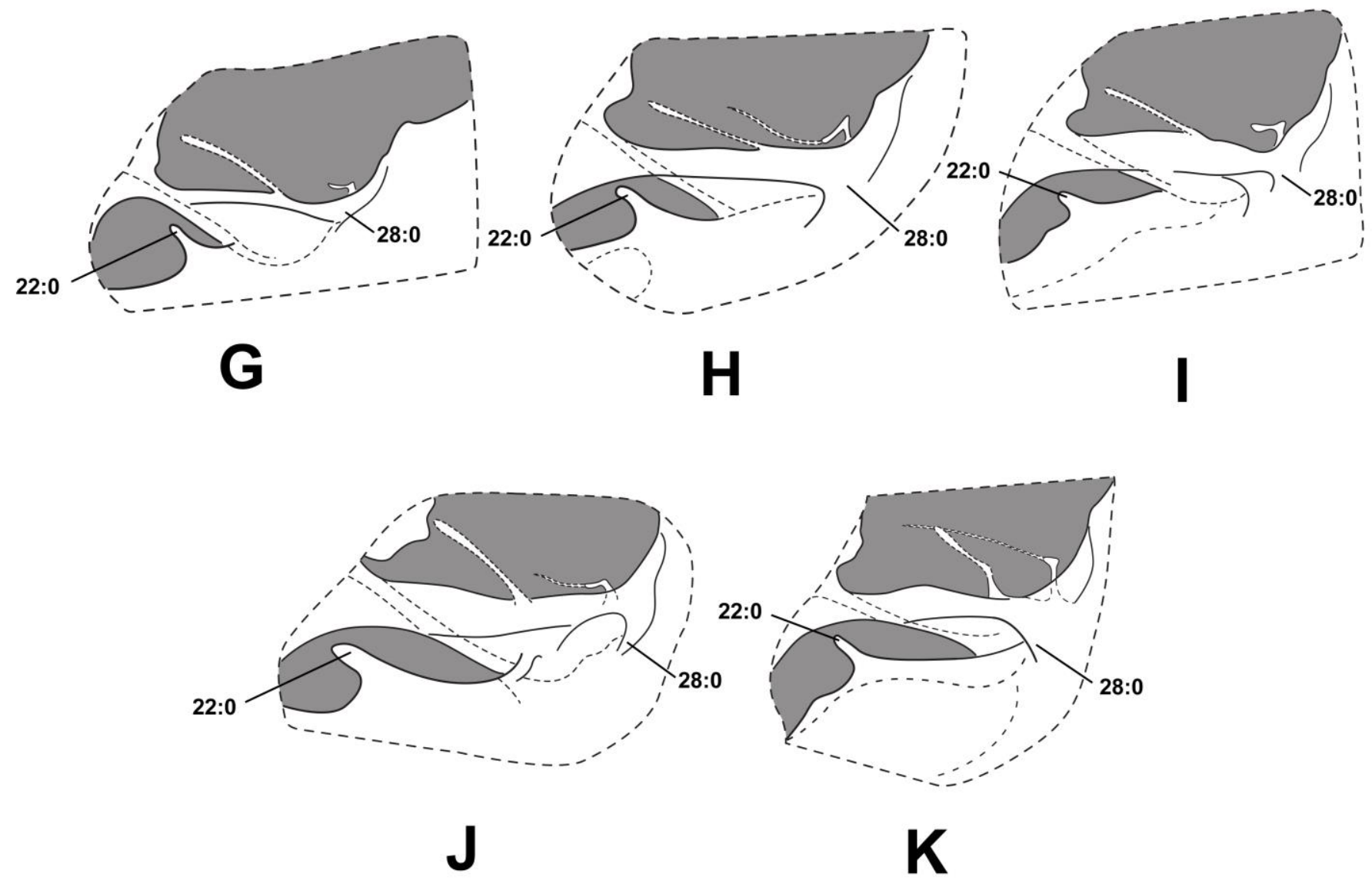
Figure 9. (next page) Tentorium; dorsal view, anterior to the top. A. Epicharis flava Friese, 1900; B. Centris analis (Fabricius, 1804); C. Euglossa melanotricha Moure, 1967; D. Bombus brasiliensis Lepeletier, 1836; E. Tetragonula carbonaria (Smith, 1854); F. Austroplebeia australis (Friese, 1898); G. Trigonisca nataliae (Moure, 1950); H. Tetragonisca angustula (Latreille, 1811); I. Scaptotrigona depilis (Moure, 1942); J. Melipona scutellaris Latreille, 1811; K. Apis mellifera Linnaeus, 1758. 


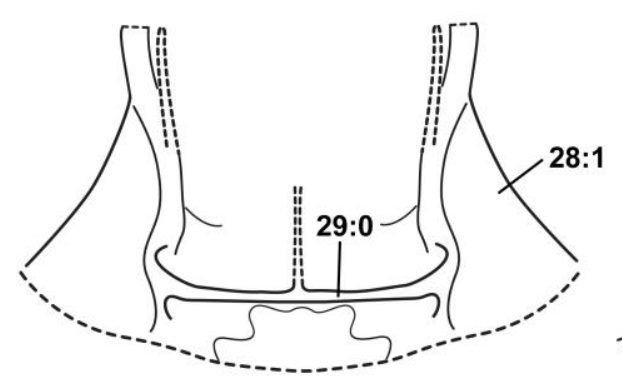

A

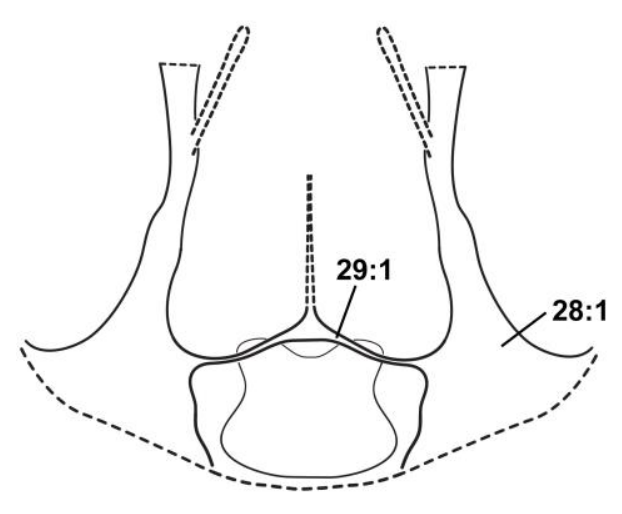

D

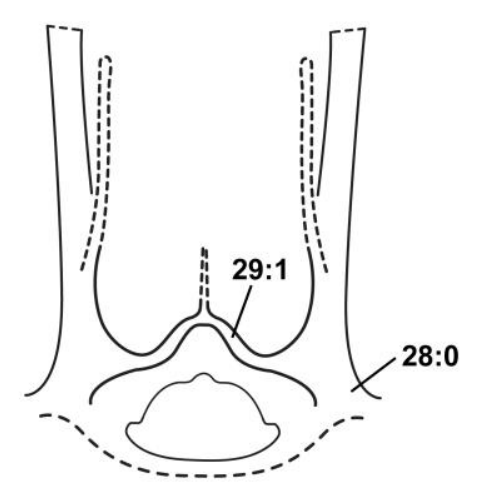

G

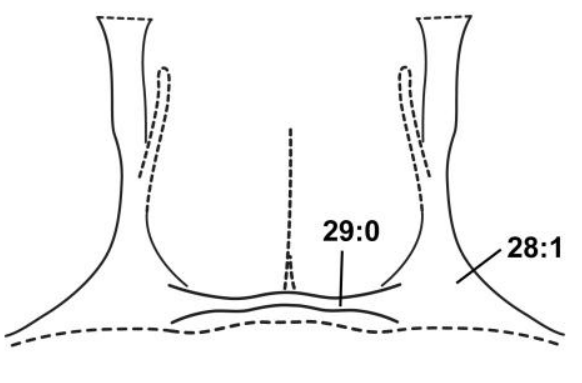

B

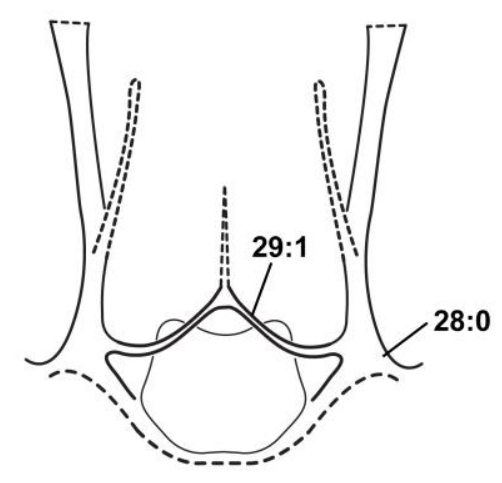

E

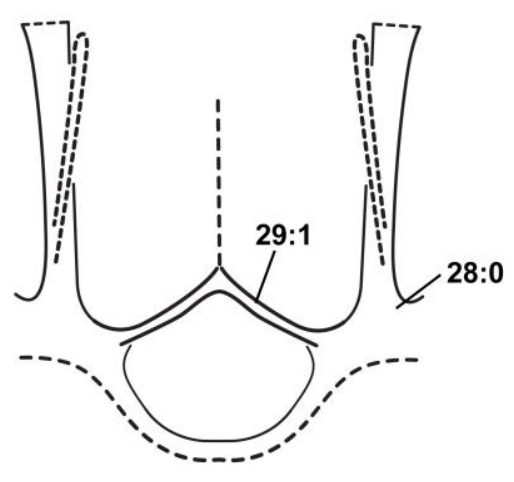

H
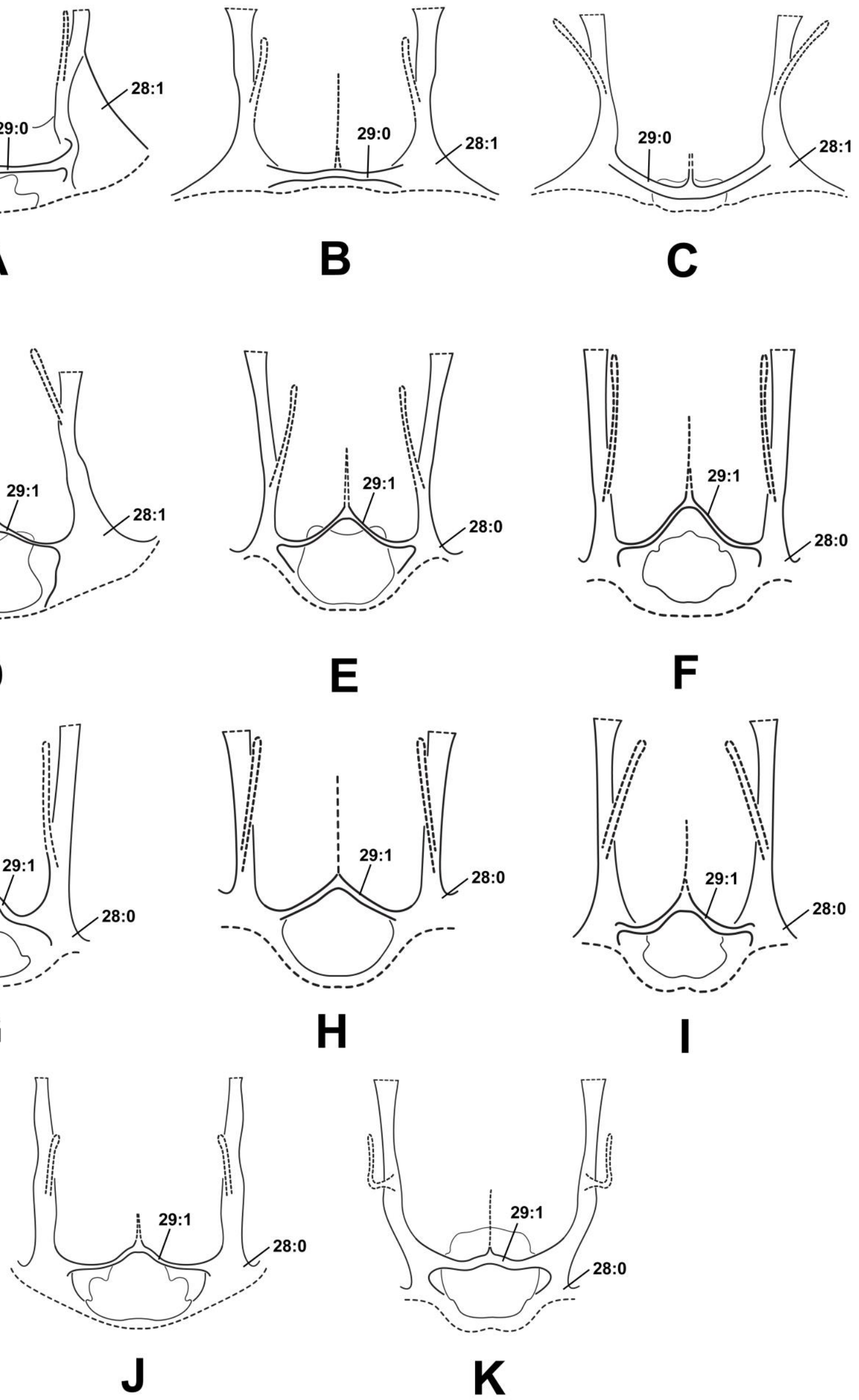
Figure 10. (next page) Sitophore and hypopharyngeal lobe; anterior view, ventral to the bottom. A. Apis mellifera Linnaeus, 1758; B. Epicharis flava Friese, 1900; C. Centris analis (Fabricius, 1804); D. Euglossa melanotricha Moure, 1967; E. Bombus brasiliensis Lepeletier, 1836; F. Tetragonula carbonaria (Smith, 1854); G. Austroplebeia australis (Friese, 1898); H. Tetragonisca angustula (Latreille, 1811); I. Scaptotrigona depilis (Moure, 1942); J. Melipona scutellaris Latreille, 1811. 

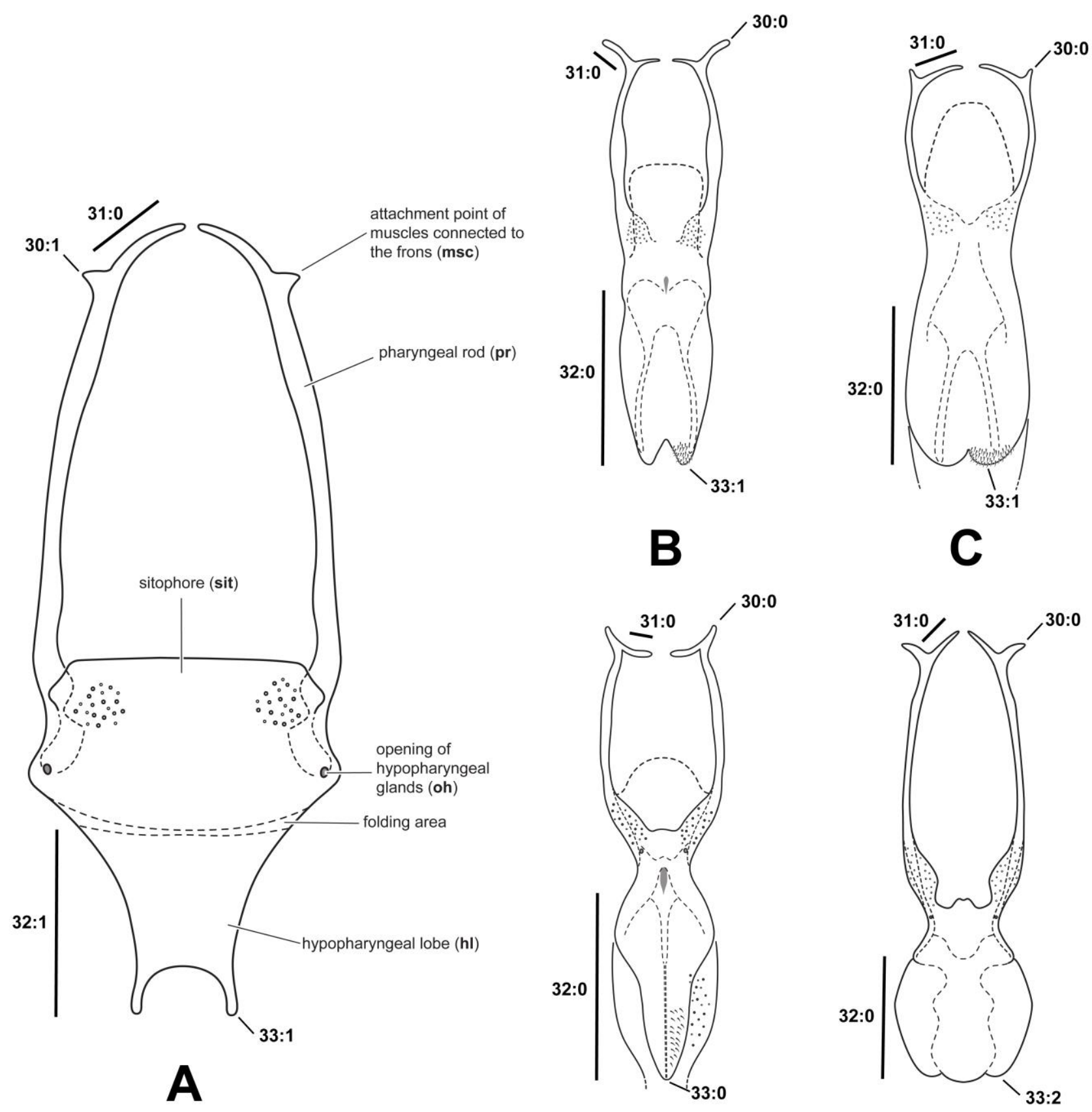

B

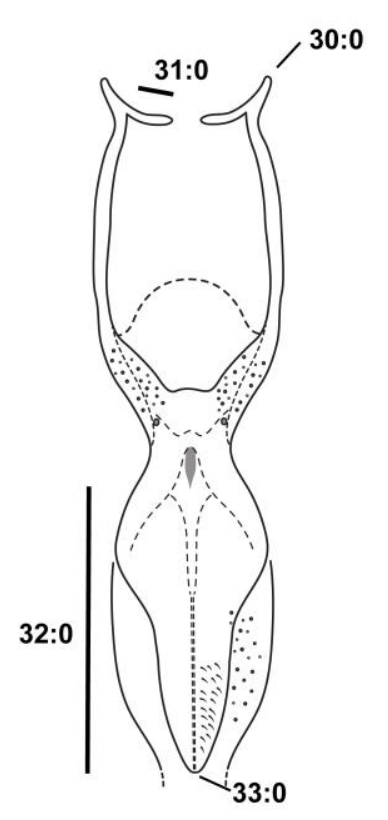

C

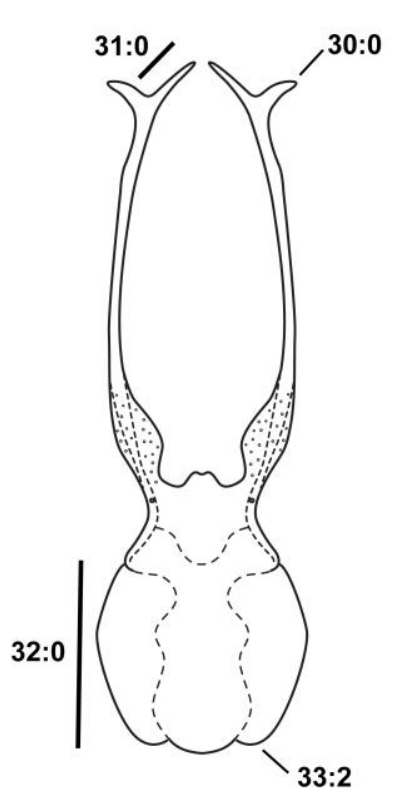

D
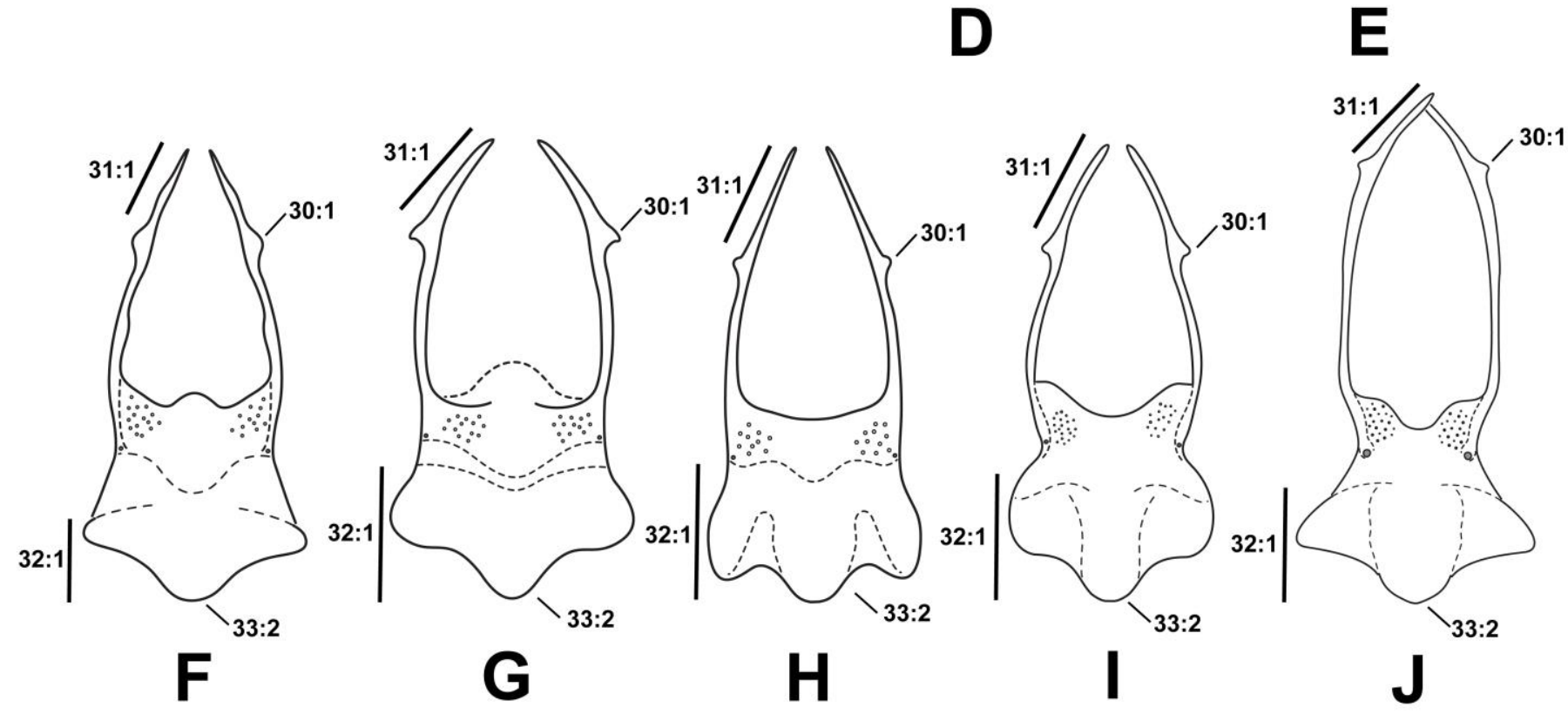


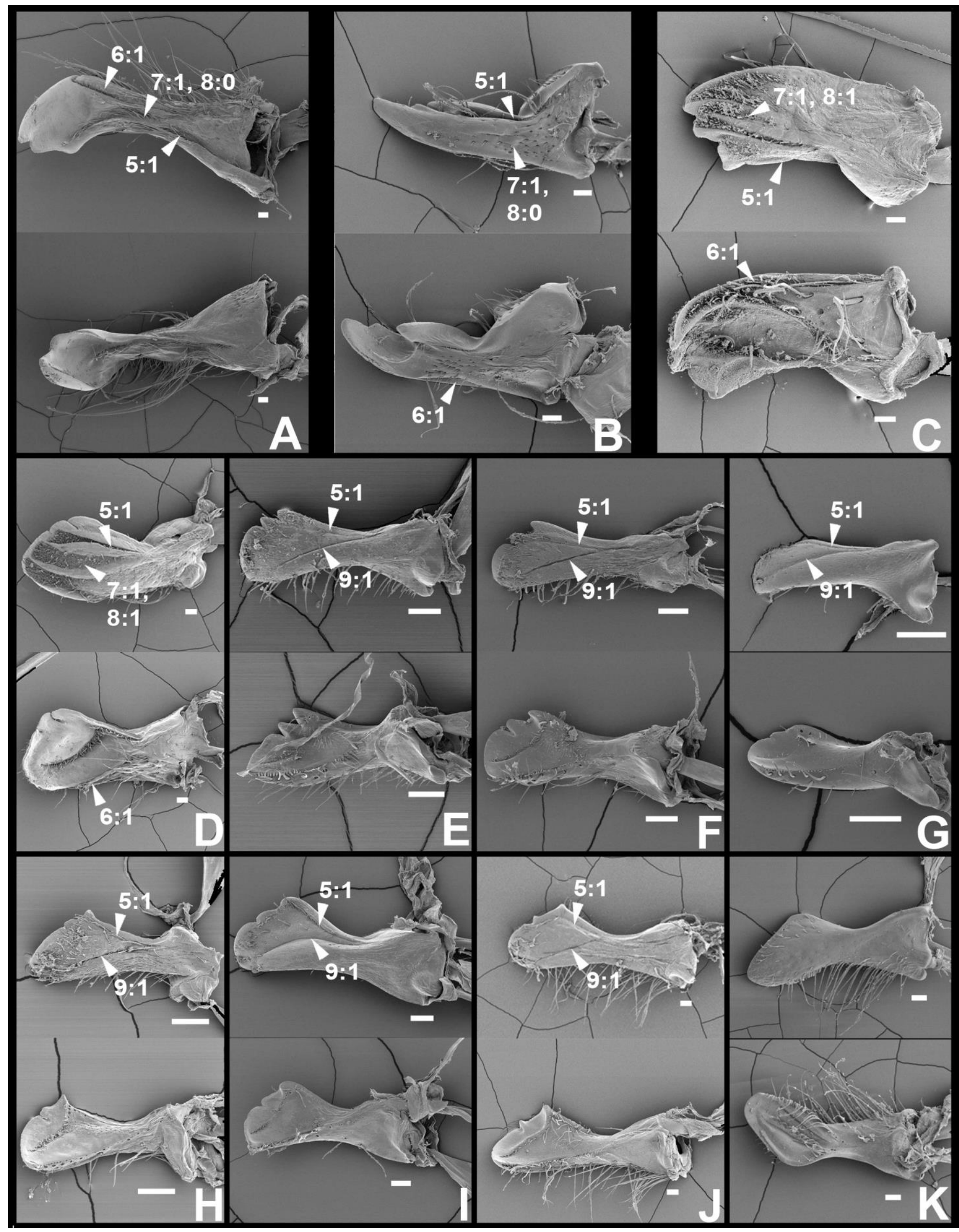

Figure 11. SEM images of the mandibles; top = external view, bottom = internal view; apex to the left. A. Epicharis flava Friese, 1900; B. Centris analis (Fabricius, 1804); C. Euglossa melanotricha Moure, 1967; D. Bombus brasiliensis Lepeletier, 1836; E. Tetragonula carbonaria (Smith, 1854); F. Austroplebeia australis (Friese, 1898); G. Trigonisca nataliae (Moure, 1950); H. Tetragonisca angustula (Latreille, 1811); I. Scaptotrigona depilis (Moure, 1942); J. Melipona scutellaris Latreille, 1811; K. Apis mellifera Linnaeus, 1758. Scale bar $=100 \mu \mathrm{m}$. 


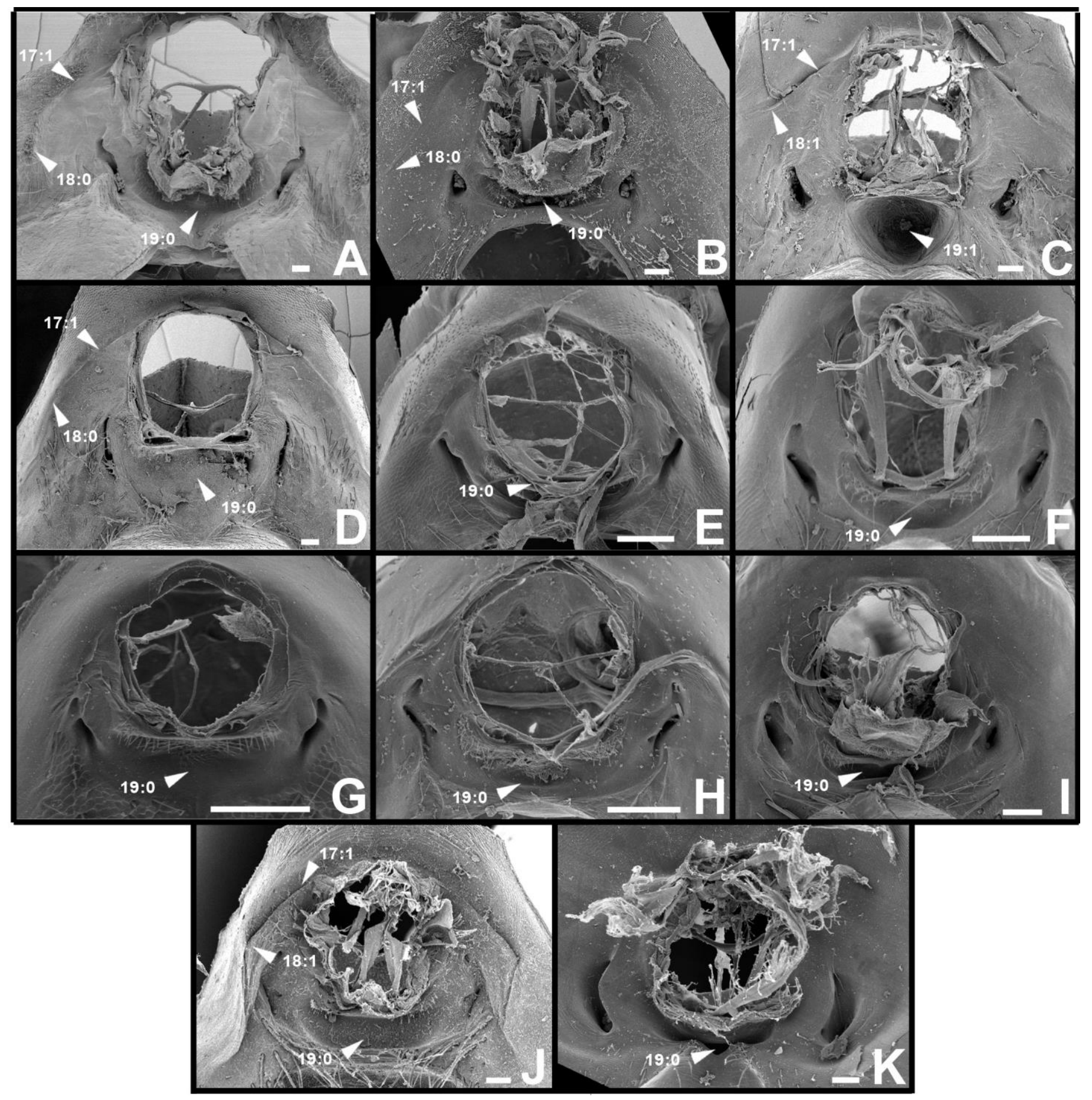

Figure 12. SEM images of the occiput and postocciput; posterior view. A. Epicharis flava Friese, 1900; B. Centris analis (Fabricius, 1804); C. Euglossa melanotricha Moure, 1967; D. Bombus brasiliensis Lepeletier, 1836; E. Tetragonula carbonaria (Smith, 1854); F. Austroplebeia australis (Friese, 1898); G. Trigonisca nataliae (Moure, 1950); H. Tetragonisca angustula (Latreille, 1811); I. Scaptotrigona depilis (Moure, 1942); J. Melipona scutellaris Latreille, $1811 ;$ K. Apis mellifera Linnaeus, 1758 . Scale bar $=100 \mu \mathrm{m}$. 


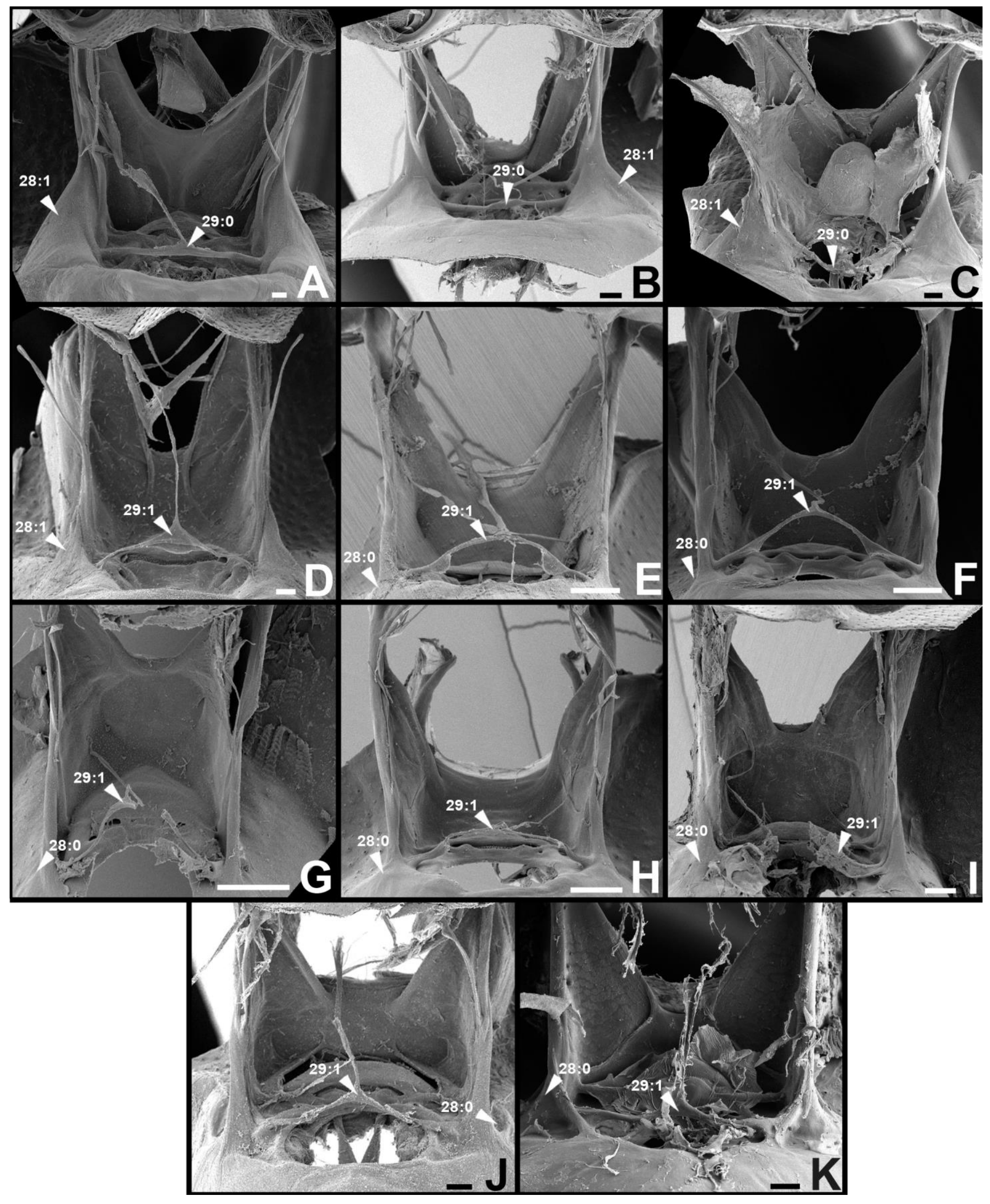

Figure 13. SEM images of the tentoria; dorsal view, anterior to the top. A. Epicharis flava Friese, 1900; B. Centris analis (Fabricius, 1804); C. Euglossa melanotricha Moure, 1967; D. Bombus brasiliensis Lepeletier, 1836; E. Tetragonula carbonaria (Smith, 1854); F. Austroplebeia australis (Friese, 1898); G. Trigonisca nataliae (Moure, 1950); H. Tetragonisca angustula (Latreille, 1811); I. Scaptotrigona depilis (Moure, 1942); J. Melipona scutellaris Latreille, $1811 ;$ K. Apis mellifera Linnaeus, 1758 . Scale bar $=100 \mu \mathrm{m}$. 


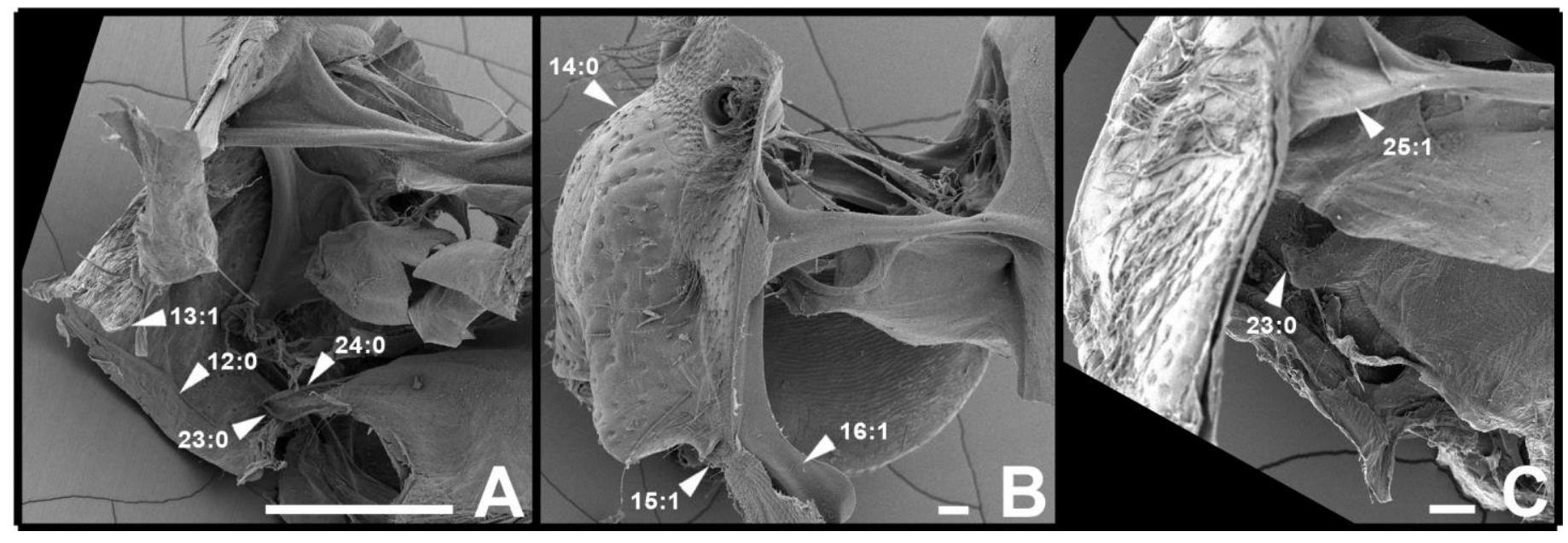

Figure 14. SEM images of the dissected heads showing the tentorium; lateral view, anterior to the left. A. Epicharis flava Friese, 1900; B. Centris analis (Fabricius, 1804); C. Euglossa melanotricha Moure, 1967. Scale bar $=100 \mu \mathrm{m}$. 


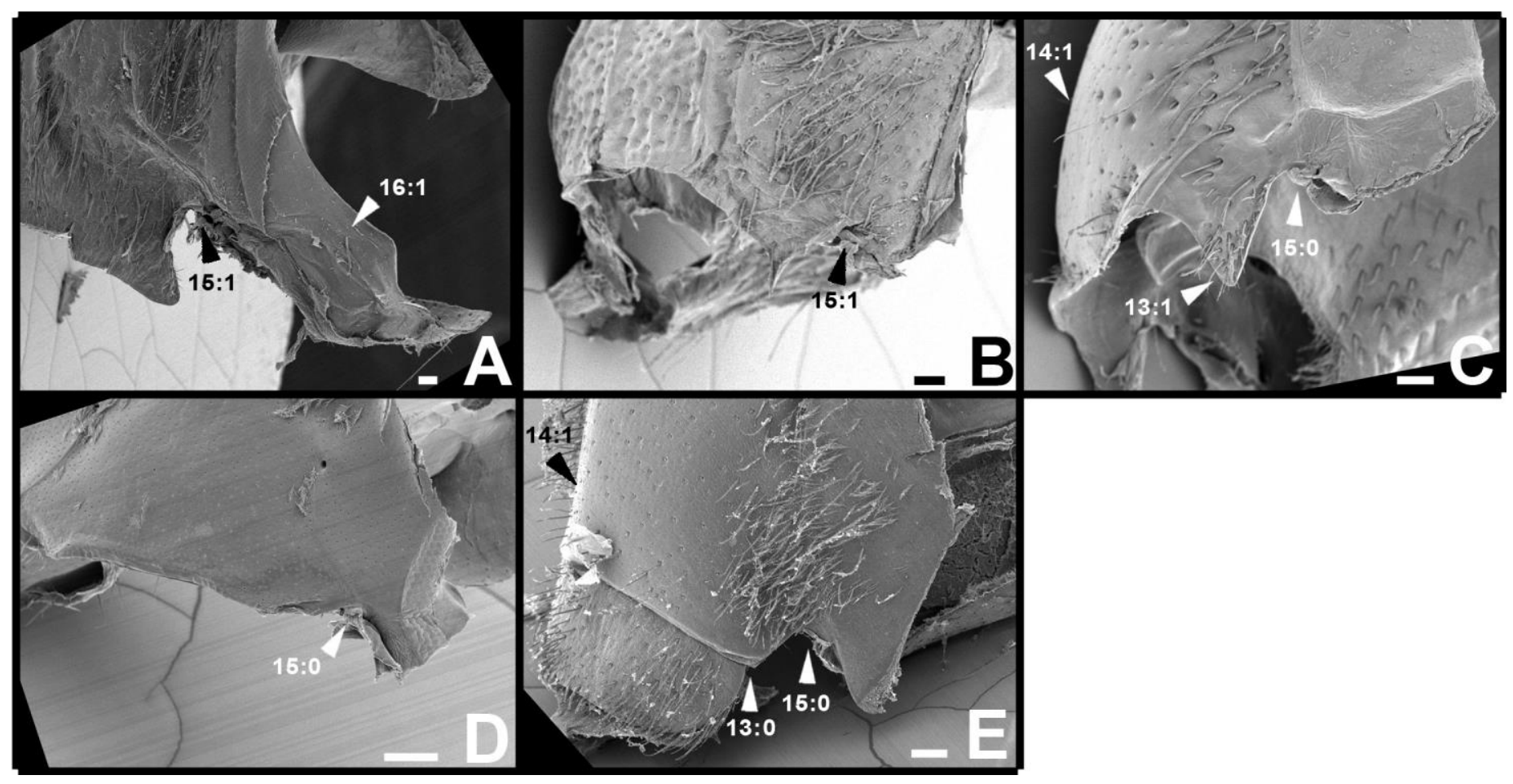

Figure 15. SEM images of the lower portion of clypeus depicting the pleurostomal condyle; lateral view, anterior to the left. A. Epicharis flava Friese, 1900; B. Euglossa melanotricha Moure, 1967; C. Bombus brasiliensis Lepeletier, 1836; D. Tetragonula carbonaria (Smith, 1854); E. Apis mellifera Linnaeus, 1758.Scale bar $=100 \mu \mathrm{m}$. 


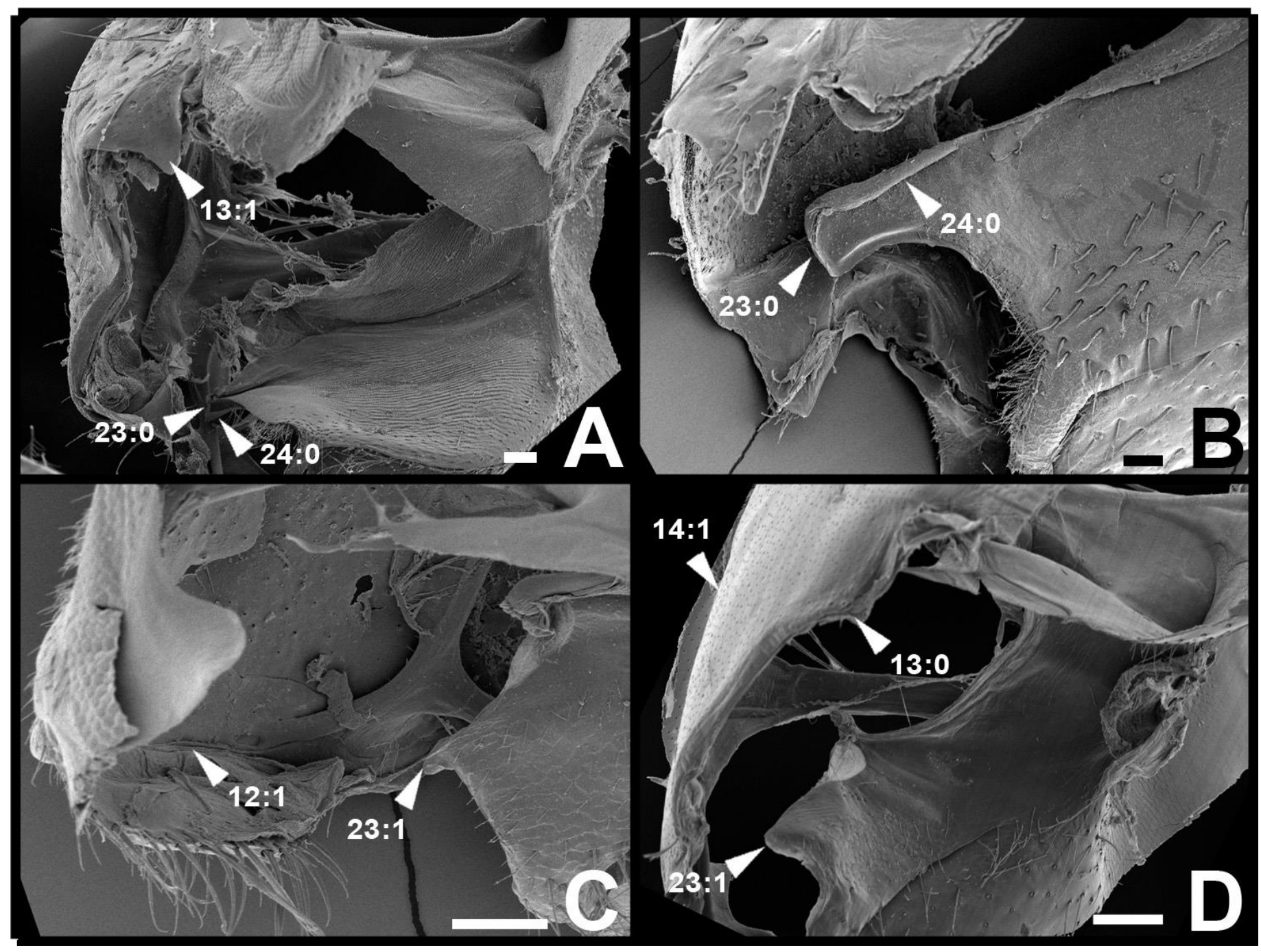

Figure 16. SEM images of the lower portion of clypeus depicting the paramandibular process; latero-ventral view, anterior to the left. A. Centris analis (Fabricius, 1804); B. Bombus brasiliensis Lepeletier, 1836; C. Austroplebeia australis (Friese, 1898); D. Tetragonula carbonaria (Smith, 1854); Scale bar $=100 \mu \mathrm{m}$. 


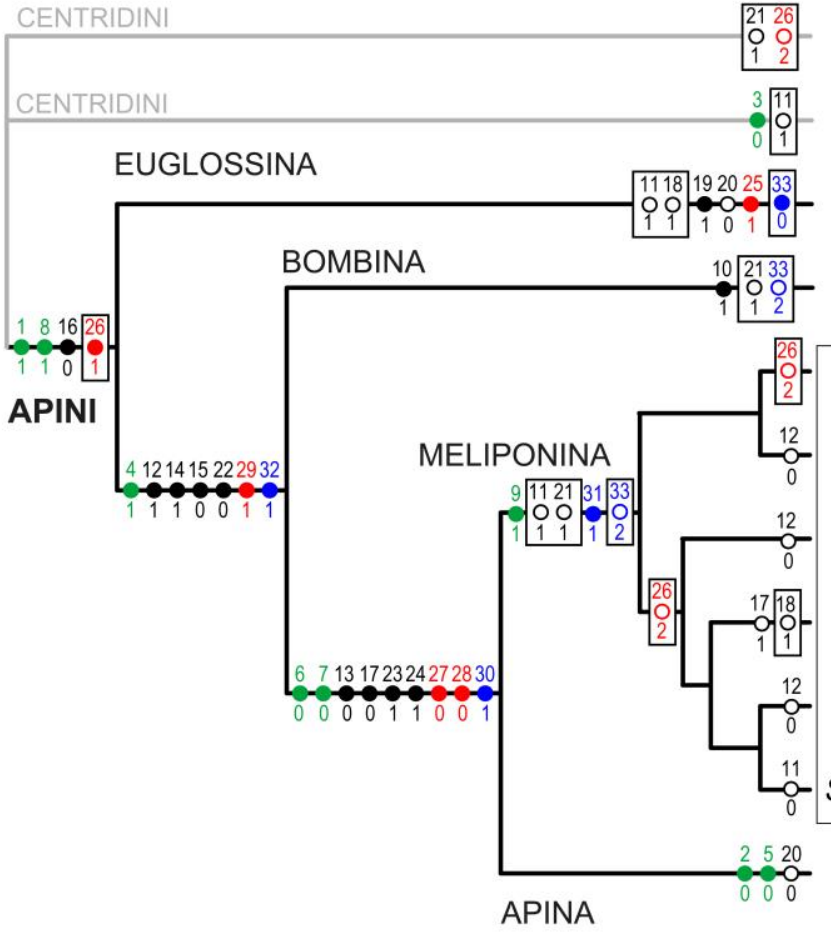

HYPOTHESIS 1
Epicharis flava

Centris analis

Euglossa melanotricha

Bombus brasiliensis

Austroplebeia australis

Tetragonula carbonaria

Trigonisca nataliae

Melipona scutellaris

Tetragonisca angustula

Scaptotrigona aff. depilis

Apis mellifera

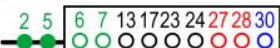

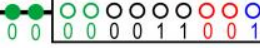

APINA

HYPOTHESIS 2

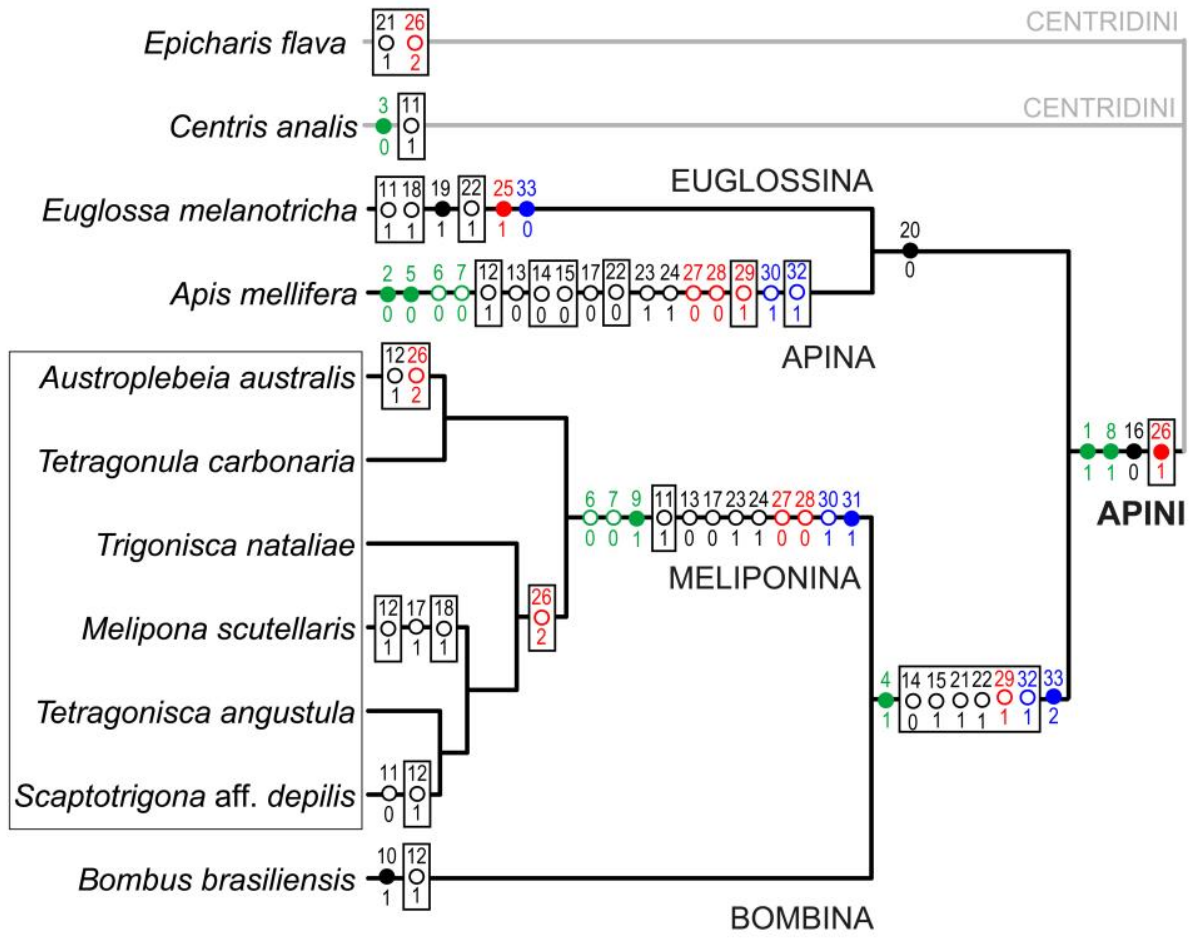

HYPOTHESIS 3

Figure 17. Mapping of character state changes onto concurrent phylogenetic hypotheses for corbiculate bees. To the left (Hypothesis 1), synoptic tree of most hypotheses based on morphological and behavioral datasets; to the right (Hypothesis 2), synoptic tree of hypotheses based on molecular datasets; to the bottom (Hypothesis 3), synoptic tree of an alternative hypothesis commonly recovered with molecular datasets. For more details on concurrent phylogenetic hypotheses for the corbiculate relationships, see Cardinal et al. (2007) and Almeida \& Porto (2014). Filled circles represent unique changes; open circles represent homoplasious changes; values above circles are character numbers; values below circles are character states changes; character state changes inside the boxes are the ones treated as delayed transformations in the optimizations (DELTRAN). The colors of the labels depict the morphological complexes represented: mandibles (=green); head capsule (=black); tentorium (=red); sitophore (=blue). 


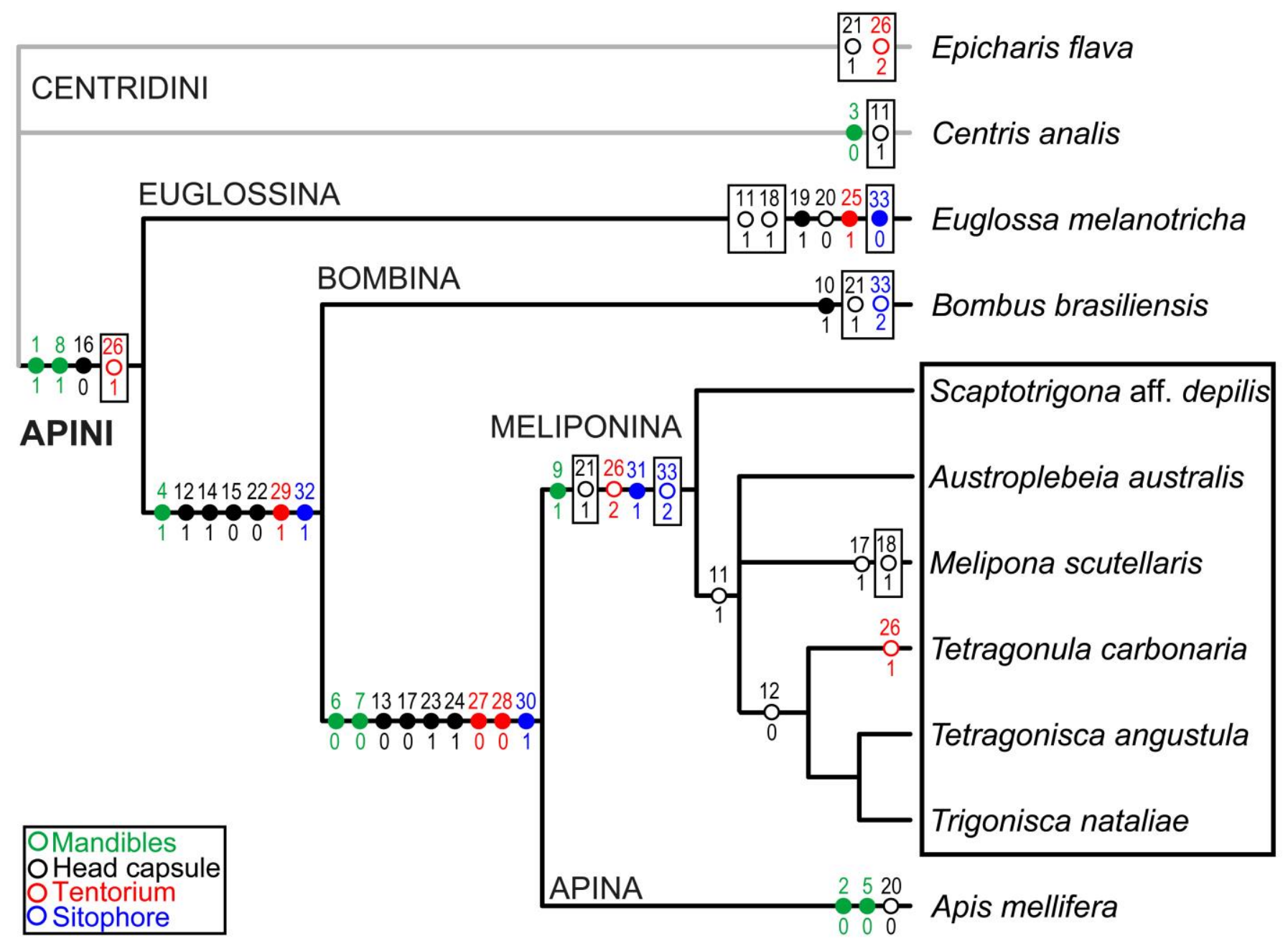

Figure 18. Single tree obtained from cladistics analysis of the morphological dataset with characters from the head, mandibles and sitophore. Open circles indicate homoplasious character-state changes; filled circles indicate unambiguous character-state changes; numbers above circles indicate the characters; character state changes inside the boxes are the ones treated as delayed transformations in the optimizations (DELTRAN). The colors of the labels depict the morphological complexes represented: mandibles (=green); head capsule (=black); tentorium (=red); sitophore (=blue). L: 46, Cl: 76, RI: 83. 


\title{
Chapter 2 - Comparative Morphology of Internal Structures of Mesosoma of Bees with emphasis in the Corbiculate Clade (Hymenoptera: Apidae: Apini)
}

\begin{abstract}
Complex internal skeletal structures, despite their potential as rich sources of phylogenetic information, still are poorly described and used in comparative studies of insects, and bees in particular. In this work, a comprehensive investigation of the internal structures of the mesosoma of bees (e.g., prosternum, propleuron, mesofurca/metafurca and mesophragma) were described and a comparative study was undertaken for bees in general with special emphasis on the tribe Apini. Twenty-eight characters are proposed and the morphological terminology used for bees is standardized to that used for other Hymenoptera. Moreover, the potential of these morphological complexes as sources of phylogenetic information was evaluated contrasting the data obtained here with alternative scenarios for bee phylogeny. Different morphological complexes of mesosoma clearly present distinct phylogenetic signals, and their significance as sources of information was not equal throughout the phylogeny of bees. The prosternum concentrated the highest number of uncontroverted character state changes - nine transformations in the most favorable scenario. On the other hand, considerable degree of plasticity was observed particularly for the changes of the character states from propleuron and mesofurca/metafurca. In part, this can be the consequence of long morphological branch or a rapid diversification of the tribe Apini. In this context, the inclusion of fossil information and use of new technologies for phenotypic studies can open new windows for future comparative morphological studies in bees.
\end{abstract}

\section{Introduction}

Bees are a morphological diverse group of aculeate hymenopterans that is included into the superfamily Apoidea (Melo, 1999). The latter is the superfamily that also includes the so called "apoid wasps" or "sphecoid wasps" (Spechidae s.l., sensu Bohart \& Menke, 1976), of which, the family Crabronidae appears to be the sister-taxa of bees (Melo, 1999). The bee clade has been referred by different authors as Apiformes or Anthophila (Brothers, 1975; Engel, 2005). Bees comprise approximately 20,000 valid species (Ascher \& Pickering, 2014) divided into seven families (Roig-Alsina \& Michener, 1993; Alexander \& 
Michener, 1995; Michener, 2007; Danforth et al. 2013), among which Apidae is one of the best-known groups. In addition to the species richness, a remarkable diversity of morphologies and social structures is known to exist within this group. One of the most fascinating and studied group of bees in this respect is that of corbiculate bees (Apidae: Apinae: Apini).

The corbiculate bee taxon constitutes a conspicuous monophyletic group that differentiated about 75 million years ago during the Late Cretaceous (Cardinal et al., 2010; Cardinal \& Danforth, 2011). The group comprises the well-known honey bees (genus Apis), bumblebees (genus Bombus), orchid bees (Aglae, Exaerete, Eufriesea, Eulaema and Euglossa) and stingless bees (c.a. 60 genera) (Michener, 2007; Ascher \& Pickering, 2014). The Apini clade provides an interesting model for the study of eusociality in bees because it contains representatives with different degrees of social organization, including the only bees that present the fixed-caste eusociality (sensu Almeida \& Porto, 2014): Apina and Meliponina (Michener, 1974, Noll, 2002). However, there is still much controversy concerning the evolution of the traits that define totipotent-caste and fixed-caste eusociality on these bee lineages because of contrasting phylogenetic results within Apini, that became known as the "corbiculate controversy" (see Chavarría \& Carpenter, 1994; Schultz et al., 1999; Ascher et al., 2001; Cardinal et al., 2007; Kawakita et al., 2008; Payne, 201; and Almeida \& Porto, 2014 for detailed discussion on the "corbiculate controversy").

The search for new phylogenetic-informative characters from novel sources is of great interest in this context. Important internal skeletal morphological complexes that still require further studies and better descriptions include the propectus, internal ridges of mesosoma, mesofurca/metafurca, prophragma and mesophragma. The prothorax, particularly the morphological unity corresponding to the propectus (i.e., propleura, prosternum and forelegs; Snodgrass, 1910), is of particular interest, once it was showed by Prentice (1991) that the shape of basisternum offers critical evidence that could help elucidate the "corbiculate controversy". Characters derived from the prosternum were first used in phylogenetic analyses of corbiculate bees by Kimsey (1984), posteriorly by Prentice (1991), and in a broader context by Roig-Alsina \& Michener (1993), and Melo (1999).

Another important morphological unity that was overlooked by most morphologists was the mesofurca/metafurca. This intricate apophyseal system was never extensively explored before in a comparative manner for bees and seems to be quite informative for 
phylogenetic purposes. The mesophragma is also notably interesting, because it is a conspicuous internal sclerite, closely associated with the propodeum, mesoscutellum, and $4^{\text {th }}$ axillary sclerite (Michener, 1944), and appears to offers variation that is congruent with some molecular hypotheses proposed for Apidae (pers. obs.). These are just some examples that illustrate the possibilities hidden inside the mesosoma.

An extensive morphological comparative study of these internal thoracic skeletal structures was never done focusing on bees as a whole. In this concern, the main objectives of this work were to describe and study comparatively the skeletal morphology of some internal structures of the mesosoma of bees (e.g., propectus, mesofurca/metafurca, mesophragma), with special emphasis in representatives of the corbiculate clade (Apidae: Apini). Also, it was attempted to access the potential of these structures as sources of information to illuminate the controversy concerning the phylogenetic relationships of the corbiculate clade. The information provided by these structures was evaluated contrasting the data obtained here with conflicting phylogenetic hypotheses published on literature.

\section{Material and methods}

\section{1. Material examined}

Specimens pertaining to 44 bee species were cleared and dissected, 25 belonging to the family Apidae (Table 1). Only female specimens were used in morphological comparisons, though males were also cleared and dissected. Representatives of all major lineages of bees, including 9 tribes of Apidae, were selected according to the geographic and phylogenetic diversity of the taxa they represent, as proposed in recent phylogenetic studies (Alexander, 1991a, b; Hines, 2008; Ramírez et al., 2010; Rasmussen \& Cameron, 2010). The sampling of groups other than Apidae was composed by 19 species of other bee families expecting to represent the morphological and phylogenetic diversity in these groups (Alexander \& Michener, 1995; Cardinal et al., 2010). The material used in this paper was obtained from Coleção Entomológica "J.M.F. Camargo" (RPSP), Departamento de Biologia, FFCLRP-USP. The classification of bee taxa followed the proposal of Michener (2007) and the status of corbiculate taxa as subtribes followed the suggestion of A. Roig-Alsina (Roig-Alsina \& Michener, 1993; p. 158; Silveira et al., 2002). All genusnames, when necessary, were abbreviated using two letters to avoid ambiguities. 


\section{2. Preparations and illustrations}

Specimens were treated with $10 \% \mathrm{KOH}$ solution, according to the protocol presented by Melo (1999), with a few adjustments. Pinned specimens were cleared by treatment for $20-45$ minutes in $10 \% \mathrm{KOH}$ solution heated at $90-100{ }^{\circ} \mathrm{C}$. Specimens fixed in alcoholic solution were boiled for 10 minutes in absolute ethanol before the procedure mentioned above. Once the sclerites have softened enough, the specimens were disarticulated using surgical tweezers and fine scalps. The propectus was separated from the head and the remainder of mesosoma. The propectus was soaked in hydrogen peroxide $3 \%$ solution heated at $90-100{ }^{\circ} \mathrm{C}$ for $1-2$ minutes and then transferred to $70 \%$ boiling ethanol for 10 minutes. After that, the propleura and prosternum were separated and the pieces transferred to a series of dehydrating solutions containing ethanol in crescent concentrations (80\%-100\%) and then placed on pure glycerin. The remainder of mesosoma was heated in hydrogen peroxide 3\% solution for a longer time, 15-20 minutes depending on the specimen. Then, the pronotum and mesoscutum/mesoscutellum were separated exposing the internal structures of mesosoma. After that, the mesosoma was submitted to the same steps described above for the propectus.

The structures were illustrated with aid of a camera lucida attached to a stereomicroscope Leica M205C with transmitted light and dark field. To hold the specimens, an excavated slide filled with a high density medium was used. After that, the

illustrations were digital-vectored using the software Adobe Illustrator CS. Digital photographs of high resolution on different focal levels were taken using a camera Leica DFC450 attached to the same stereomicroscope and assembled using LAS Montage module. Photographs were treated in the software Adobe Photoshop CS4.

\section{3. Morphological terminology}

The morphological terminology referring to general structures of mesosoma followed Michener (1944) as a fundamental basis. The studies of Camargo et al. (1967), Roig-Alsina \& Michener (1993) and Melo (1999) were also useful, especially the latter two, because they discussed comparative morphology with a phylogenetic background and provided valuable information about internal structures of bees as well. An attempt to standardize the specific terminology used for bees to that used for other Hymenoptera was made using the works of Snodgrass (1910, 1942, 1956), Vilhelmsen (2000a, b), Vilhelmsen et al. (2010) and the HAO web portal (Yoder et al., 2010) as references. The 
terms used to define particular morphological units of interest were indicated in bold when cited on the text for the first time.

\section{4. Character study}

The construction of morphological characters was conducted in two main steps. Initially, the observed variation was coded as distinct character states for a given structure or region, so as to encompass the disparity in morphology (e.g., shapes, forms, pubescence, etc.). For the two characters describing continuous variation (chars. 14-15), the consistency of the character states was further evaluated by measuring the structures of interest to judge whether discontinuities marked those proposed states. Character states are described in terms of their general appearance, but the range of continuous variation found for each of them is provided in the comments of each character description. When two classes (states) were recognized in the variation of measures, those were described as 'slender or broad' (char. 15); with three states, the coding was 'low, moderate, high' (char. 14). These characters were treated as ordered.

Character state changes were optimized onto different phylogenetic hypotheses to see how the characters of the mesosoma can be interpreted in light of other phylogenies produced with broader datasets. Character states distribution and optimizations were accessed with the WinClada software (Nixon, 2002). The trees used to the optimizations were built according to recent reviews on the phylogenetic relationships of bees (Cardinal \& Danforth, 2013; Danforth et al., 2013), which places Melittidae as sister to all other bees. For the phylogenies of Euglossina and Meliponina, the works of Ramirez et al. (2010) and Rasmussen \& Cameron (2010), respectively, were taken. Alternative views on the subtribal relationships of corbiculate bees were based on summaries of previous works with morphological, behavioral and molecular datasets (see the works of Cardinal et al., 2007 and Almeida \& Porto, 2014)

\section{Results and discussion}

\section{1. Morphological descriptions}

This section provides brief descriptions on the internal structures of mesosoma under study (i.e., propectus, mesofurca/metafurca, and mesophragma). To more detailed and exhaustive treatments on internal morphology of bees see the works of Snodgrass $(1942,1956)$, 
Michener (1944), and Camargo et al. (1967). The terminology used for the morphological descriptions of some structures of the mesosoma of bees is standardized and correlated to more general terms used for other Hymenoptera. The standardized terminology is given on Table 2.

\section{1. 1. General morphology of the propectus}

The prothorax of bees corresponds to a small segment relative to the other thoracic segments. It is located anteriorly in the mesosoma and provides an articulation between the head capsule and the remaining of the body. It is constituted by the ring shaped pronotum, rigidly connected to the mesonotum, and by the propectus, which comprises the lateroventral propleura, the ventro-median prosternum and the forelegs (Snodgrass, 1910). This structure was thoroughly described by Snodgrass (1956) and Michener (1944) and will be just briefly described here.

\section{Prosternum (Figs. 1, A-E; 4-5: 1-44)}

The prosternum (Fig. 1, A-E) is an intricate structure located on the ventro-median portion of the prothorax, flanked by both propleura. It is constituted by an external portion, represented by the basisternum plus furcasternum (Fig. 1, A), and a complex internal portion, the profurca (Fig. 1, B-E). The external portion is margined anteriorly by the conjunctive membrane that originates at the median and posterior inflections of the ventral propleural area; laterally by the conjunctive membrane that surrounds the procoxal foramina; and posteriorly by the latero-ventral extensions of the pronotum. The profurca occupies partially the internal space of the prothorax, most filled with muscles, and its profurcal arms (Fig. 1, B-E) extend, at least in part, posteriorly into the mesothorax.

The anterior portion of the external surface of the prosternum corresponds to the basisternum (Fig. 1, A). The anterior angle of this region is produced anteriorly forming the anterior process of the prosternum (Fig. 1, A) and the lateral angles, somewhat projected, form the lateral processes of the prosternum (Fig. 1, A). The general shape and development of these processes are much variable (Fig. 4: 1-44) and will be further discussed in the section on comparative morphology. These processes and a considerable area of the anterior portion of basisternum are placed beneath the propleura. The posterior limits of the basisternum are defined by the profurcal pit and by the basisternal carina (Fig. 1, A). The profurcal pit is a deep groove resulting from the inflection of the cuticle 
that originates the profurca. The depth and extension of this groove are variable in distinct groups (Fig. 4: 1-44) and, sometimes, very shallow or absent (Fig. 4: 30-44). The basisternal carina normally is well developed and extends from the median line of the basisternum, above the profurcal pit, to close to the lateral processes. It presents a conspicuous emargination medially, whose shape and depth differs considerable among groups (Fig. 4: 1-44), and becomes sinuous antero-laterally at both sides, disappearing nearby to the lateral processes. The carina is partially or fully absent in a few groups, like most Apini (Fig. 4: 30-44). This carina, as mentioned by Michener (1944), fit the basal portions and median margins of the procoxa in repose. In the majority of the groups studied, the whole surface of the basisternum is covered with bristles (Fig. 4: 36-41, 43-44), especially the region of the profurcal pit, including simple and plumose types of bristles.

The posterior portion of the external surface of prosternum comprises the furcasternum (Fig. 1, A). This region corresponds to the main part of the prosternum and is the bearer of the profurca (Fig. 1, B). The furcasternum is a somewhat rectangular plate that is anteriorly delimited by the basisternal carina and profurcal pit, extends posteriorly with nearly parallel lateral margins, and terminates at a broadly rounded posterior margin. Its external surface is demarcated medially by a longitudinal groove, the median prodiscrimen (Fig. 1, B), that extends from the profurcal pit to close to the posterior margin of prosternum. This groove indicates externally the line of attachment of the prodiscrimenal lamella (Figs. 1, C-D). The median region of the external surface is frequently covered with simple and plumose bristles, which in some groups as the Centridini (Fig. 4: 27-29) are strong and occupy densely the major part of the furcasternum. In a few studied specimens another conspicuous feature could be observed on the surface of the furcasternum. In some groups, there is also a pair of carinas that meet the basisternal carina at both sides of the profurcal pit (Fig. 4: 6, 9, 11-16). These carinas probably represent posterior extensions of the basisternal carina that crosses above the profurcal pit and follows on the surface of the furcasternum. The surface between these two carinas, when they are present, is slight concave and concentrates the majority of bristles of the furcasternum.

The profurca (Fig. 1, B-E) comprises the largest portion of the prosternum, which is hidden inside the prothoracic cavity and provides the sites of origin and insertion of many muscles responsible for the movements of the head capsule, procoxa and propleura (see Vilhelmsen, 2000a,b for more details on prothoracic musculature). The whole structure is 
composed by a basal portion connected to the internal surface of the furcasternum and by two distal profurcal arms (Fig. 1, B-E).

The basal portion of the profurca presents a "box-like" organization that is responsible for maintain the integrity of the entire profurcal structure. The box is formed by a median vertical plate, the prodiscrimenal lamella (Fig. 1, C-E), which supports a horizontal platform, the horizontal plate of the profurca (Fig. 1, C-D). The plates are fused perpendicularly along their lengths and expand their anterior ends forming a sheetlike structure that between the two diverging profurcal arms, flanked by the ventral profurcal lamellas (Fig. 1, D). This sclerotic sheet converges again anteriorly and continues as a heavy sclerotic median ridge on the internal surface of the basisternum. This ridge was referred as the "median margin of the basisternum" by Camargo et al. (1967), but the use of the word "margin" seems strange considering that it constitutes a heavy sclerotic bar or ridge, not a true margin. This structure, in fact, represents the anterior extension of the prodiscrimenal lamella on the internal surface of the basisternum (Fig. 1, C). The remaining parts of the box-like structure are formed by the inflections of the lateral and posterior margins of the furcasternum, which are contiguous with the horizontal plate of the profurca and forms the posterior vertical plate of the profurcal arms (Fig. 1, E). This plate, as already acknowledge by Michener (1944), closes the lateral and posterior portions of the box, thus leaving just two anterior openings, one on each side of the prodiscrimenal lamella, beneath the horizontal plate of the profurca.

The profurcal arms (Fig. 1, C-E) are the two arched divergent braces that arise from the box-like platform provided by the horizontal plate and expand into the prothoracic and mesothoracic cavities. Each arm is formed by two sclerotic blades, an inner and an outer one, which are convergent at the posterodorsal profurcal apodeme (Fig. 1; C, E) delimiting a broad arched concavity between them. The two blades expand towards the antero-dorsal direction inside the prothoracic cavity and form three distinct anteriorly oriented processes. The outer blade is the outer profurcal lamella (Fig. 1, C). This blade is anteriorly protruded at two points - a relatively small digitform dorsal process, the anterior process of the dorsal profurcal lamella (Fig. 1, C-E) and a broadly rounded median sheet-like process, the ventral profurcal lamella (Fig. 1, C-E ). These processes, especially the latter, exhibit much variability among the examined taxa (Fig. 4: 1-44). The external surface of the outer profurcal lamella, in the region comprised between the anterior process of the dorsal profurcal lamella and the ventral profurcal lamella, contains 
the point of articulation with the posterior process of the dorsal propleural ridge, thus the articulation with the propleura (Fig. 1, C). This articulation is somewhat rigid and the posterior process is restricted by a sinuous ridge at this point, the lateral profurcal ridge (Fig. 1, C). The inner blade forms the inner profurcal lamella (Fig. 1, C-E). It is normally smaller than the outer one and is conspicuously produced anteriorly forming the anterior profurcal apodeme (Fig. 1, C-D) that originates the thin tendon of the profurcolaterocervical muscle (Vilhelmsen, 2000b). The inner lamellas of the adjacent profurcal arms can be free or, frequently, fused. When the latter occurs, they close the neural foramen of the profurca above the horizontal plate forming the profurcal bridge (Fig. 4: 4-20, 22-25, 30-33, 36, 38-39, 43-44). This bridge sometimes presents a median dorsal lamella, the median profurcal lamella, which represents the dorsal extension of the fused inner profurcal lamellas.

\section{Propleuron (Figs. 2, A-D; 6-8: 1-44)}

Each propleuron (Fig. 2, A-D) corresponds to an approximately irregular pentagonal sclerite, folded about 90 degrees at a longitudinal axis crossing throughout two of its five corners. This axis subdivides the sclerite and defines two main portions - the lateral propleural area (Fig. 2, A), nearly vertical, and the ventral propleural area (Fig. 2, C), nearly horizontal. The lateral propleural area remains broadly hidden by the pronotum, whereas the ventral propleural area is exposed, and partially conceals the anterior portion of the prosternum.

The lateral propleural area presents a dorsal margin, the dorsal propleural margin, which is nearly straight and extends from the cervical prominence (Fig. 2, D), anteriorly, to the propleural arm (Fig. 2, A), at the most posterior portion of the sclerite. Internally following the dorsal margin there is a strong ridge, the dorsal propleural ridge (Fig. 2, AB). This ridge follows in parallel the dorsal propleural margin for the posterior two thirds when the latter becomes sinuous at the anterior third and projects dorsally forming a sheetlike lamella, the anterior lamella of the dorsal propleural margin (Fig. 2, A). This lamella, however, is not too much prominent in some groups (Fig. 6: 1, 17-20). The region of the lamella frequently has dense punctuation, generally presenting abundant and robust bristles with developed plumosity. The dorsal propleural ridge articulates with the prosternum trough a protuberance located on its posterior apex, the posterior process of the dorsal propleural ridge (Fig. 2, B). 
The propleural arm (Fig. 2, A) is located on the region of the posterior apex of the dorsal propleural margin, at the joining of the latter with the posterior margin of the lateral propleural area. The structure formed is an elongated projection, sometimes sheet-shaped, directed posteriorly and bent approximately 120 degrees with the main longitudinal axis, sometimes slightly rotated outwards. The propleural arm is continuous internally with the dorsal propleural ridge and with the propleural ridge forming an extension of these ridges, the ridge of the propleural arm (Fig. 2, A). It sometimes presents a sheet-shaped posterior portion, the sheet of the propleural arm (Fig. 2, A), which differs in development in different groups.

The posterior margin of the lateral propleural area follows from the point where the propleural arm inserts, towards the ventral propleural margin. The posterior margin is variable in its upper half, becoming more sinuous on the lower part and presents near its inferior extremity a process that forms the articulation with the procoxa, the lateral coxal condyle of the propleuron (Fig. 2, A). Internally, there is a strong ridge continuous with the ridge of the propleural arm and the dorsal propleural ridge. This ridge was called "pleural ridge I" by Michener (1944) although he had raised doubts about the identity and homology of this structure. Vilhelmsen (2000b) gives a proper discussion on the homology and composition of the different parts that constitute the sclerite of the propleuron. According to his work, the propleuron in Hymenoptera is a composite sclerite, constituted by the laterocervicallia fused to true pleural elements (i.e., proepimeron) and the position and presence of a propleural sulcus and ridge seems hard to accurately identify in Apocrita. However, following a conservative view and using a terminology consistent with the prefix for structures pertaining to the prothorax this ridge will be referred herein as propleural ridge (Fig. 2, A). This ridge is formed by the inflection of the cuticle, producing an internal reinforcement of the sclerite that is demarcated externally by the propleural sulcus (Fig. 2, A). The ridge is also continuous with the inflections of the posterior margin of the ventral propleural area.

The ventral propleural margin defines the inferior limits of the lateral propleural area. It represents the longitudinal axis at which the sclerite is bent medially and defines the lateral margin of the ventral propleural area as well. The margin is usually sharply delimited by a carina, the propleural carina, strong developed into a lamella in some groups (Fig. 6: 1-3, 5-9, 11-14, 16), or poorly defined to almost absent in others (Fig. 6: 30-44). The ventral propleural margin normally is uniformly curved along its extension 
and follows from area the rise of the cervical prominence, anteriorly, to the region of the lateral coxal condyle, posteriorly. Even when the carina is present and well developed, it fades out before reach the limits of the anterior and posterior regions of the ventral propleural margin.

The ventral propleural area (Fig. 2, C) has a four or five sided shape. It has three free margins, an anterior, a median and a posterior plus a lateral one that corresponds to the ventral propleural margin, as mentioned above. The free margins, actually, do not correspond to the true margins of the sclerite. They are internally deflected and these inflections (Fig. 2, B) join to the conjunctive membrane which, in turn, is attached to the margins of the prosternum, procoxa and neck. When the propleural carina is produced into a lamella a profound groove is frequently present on the median portion of the ventral propleural area and goes in parallel with the former. The entire external surface of the ventral propleural area is provided with punctuation (i.e., sockets of bristles), but the marginal areas contain the larger ones and in a higher density than the other areas.

The anterior margin of the ventral propleural area (Fig. 2, C) is nearly straight in most specimens. This margin and a short adjacent area, the anterior bar of the ventral propleural area, are commonly raised in relation to the rest of the plate and in many groups a more or less profound groove develops between them and the remaining ventral area, the propleural cervical groove. When well developed, the groove is usually delimited anteriorly and, less frequently, posteriorly by carinas. The groove and the area close to the anterior margin are often densely covered with strong plumose bristles.

The median margin of the ventral propleural area (Fig. 2, C) is usually straight and follows in parallel the corresponding margin of the opposite propleuron, concealing the anterior portion of the prosternum. The inflection of this margin provides part of the conjunctive membrane that attaches to the region of the anterior process of the prosternum.

The posterior margin (Fig. 2, C) is more variable than the others. In some groups it is straight or slightly convex; in others, emarginated, and in a few other species, more sinuous or even medially angulate. Its inflection provides the conjunctive membrane that attaches to the anterior portion of the basisternum and to the anterior portion of the procoxal foramen.

In some groups, an unusual structure is present on the ventral propleural area, the sclerotic ring (Fig. 2, B-C). The ring is formed at the region of the meeting of the inflection of the median margin and the propleural cervical groove. Another conspicuous 
feature present only in one specimen pertaining to the halictid clade is a ridge located on the median portion of the propleuron (Fig. 7: 9). This internal ridge follows obliquely from near the insertion of the cervical prominence, turns close to the median margin of the ventral propleural area, becomes parallel to its median inflection and follows it for the most part of its length, finally vanishing near the posterior margin of the ventral propleural area. The median margin of the ventral propleural area in this specimen is raised above the level of the remaining sclerite, concealing a profound median groove.

The cervical prominence (Figs. 2, D; 8: 1-44) is a complex structure that deserves separate comments. This is the structure that provides the articulation between the head capsule and the prothorax. The prominence is located at the anterior angle of each propleuron, near the junction of the most anterior extremity of the lateral and ventral propleural areas. The basal portion of this structure is strong reinforced by remnants of the dorsal propleural ridge and by the inflection of anterior margin of the ventral propleural area. The distal part forms a prominent protuberance that contains an anterior projection that articulates with the post-occipital region of the head capsule, the cranial condyle of propleuron (Fig. 2, D). The anterior and lateral surfaces of the protuberance, the cervical swelling (Fig. 2, D), are covered with strong sensorial bristles and the entire region is referred as the "Lindauer's Organ" by Camargo et al. (1967). The cervical prominence presents a posterior projection, the cervical apodeme (Fig. 2, D), that connects to the muscles pronoto-, profurco-, and procoxo-laterocervical muscles (Vilhelmsen, 2000b). The cervical apodeme is referred as the "posterior ramus of the occipital process" by Michener (1944). The apodeme is flat and create a sheet-like projection that is nearly horizontally oriented and is directed posteriorly, inside the prothorax. It normally presents sclerotic reinforcements directed anteromedially and posteriorly, the anteromedian and posterior bars of the cervical apodeme (Fig. 2, D). A cuticular sheet between them, forming a more or less quadrangular plate, is commonly present, the sheet of the cervical apodeme (Fig. 2, D). In a few species this sheet is not well developed and the major structure is solely composed by the two sclerotic bars (Fig. 8: 34, 39). The homology of the cervical prominence was previously discussed by Crampton $(1909,1926)$, which proposed that the whole region of the propleuron that carries the prominence would be homologous to the cervical sclerites (=laterocervicallia), as it occurs in lower insects, and the whole propleuron, thus of pleural and cervical origin. Michener (1944) also adopted this interpretation, although Snodgrass (1910) and Weber (1925) regarded the entire sclerite as 
originated only from pleural elements. The composite nature of the entire "propleuron" in Hymenoptera was clarified in the work of Vilhelmsen (2000b).

\section{1. 2. Mesofurca and metafurca (Figs. 3, A-B; 9-10: 1-44)}

The morphological complex comprised by the mesofurca and metafurca of bees is a very complicated unity, whose structure was discussed with some detail by Snodgrass (1910, 1942, 1956) and Michener (1944), but has received too little attention despite its potential as an interesting source of phylogenetic information in other hymenopteran groups, especially in non-aculeate taxa (Vilhelmsen, 2000a; Vilhelmsen et al., 2010). The mesofurca and metafurca of bees, as acknowledged by other authors ( $c f$. Snodgrass, 1956; Michener, 1944), present their arms fused together forming an integrated unity and, thus, will be discussed herein in conjunction.

The complex of the meso/metafurca (Fig. 3, A-B) is structured as a suspended platform supported by four sclerotic pillars from which two pairs of arms diverge anterolaterally and postero-laterally. The floor of the platform comprises a more or less rectangular plate, the horizontal plate of the mesofurca (Fig. 3, A). The lateral margins of this plate run from close to the point of insertion of the first pair of sclerotic pillars to the internal ridge that lines the anterior foramen of the mesothorax. The margins normally follow a semi-parallel trajectory on the posterior half of the plate and become more divergent anteriorly. In many groups, however, the margins are strongly divergent for almost its entire extension and the shape conferred to the plate in dorsal view is quite variable amongst distinct taxa (Fig. 9: 1-44). Crossing the median line of the horizontal plate of the mesofurca there is a lamella, that is commonly strongly developed into a crest, the mesodiscrimenal lamella (Fig. 3, A). It corresponds to the internal manifestation of the mesodiscrimen.

The sclerotic pillars that support the platform are the two anterior and two posterior free basal portions of mesofurcal and metafurcal arms (Fig. 3, A-B), respectively. They arise from the ventral horizontal plate of mesofurca and grow dorsally, until the basal portions of mesofurcal and metafurcal arms on each side fuse together and form the coalesced furcal arms (Fig. 3, A-B). The horizontal platform that connects both sides of the furcal elements is the mesofurcal bridge (Fig. 3, A). The bridge is inserted into the furcal structure close to the site of attachment of the coalesced furcal arms and its shape in dorsal view is much variable, especially due to the contours of its anterior margin (Fig. 9: 
1-44). Located at the antero-lateral corners of the bridge are the two round apodemes, whose shape and development are also quite variable. Snodgrass (1942) described in Ap. mellifera a pair of processes present on the antero-median portion of the mesofurcal bridge (Fig. 16, H, q). These processes give rise to the attachment of the ventral intersegmental muscles between the prothorax and mesothorax. In the dissected specimens of this species used in the present study, only feebly sclerotized membranous lamellas (not represented in the illustrations) were present. If these processes are to be regarded the homologous of the round apodemes or they are a particular feature of Ap. mellifera will depend on detailed examinations of more exemplars. The dorsal surface of the bridge frequently has two median impressions that sometimes delimit a more or less triangular median area, the triangular median impression of mesofurcal bridge (Fig. 3, A). This impression seems to be the result of the insertion of muscles that arise from the mesofurca and, frequently, very thin sclerotic lamellas, almost membranous, can be seem on both sides of the triangular median area in cleared specimens.

The two pairs of distally divergent arms are the free distal portions of mesofurcal and metafurcal arms (Fig. 3, A-B). They raise from the coalesced furcal arms and the elements on both sides of the mesofurca and metafurca diverge from each other anterolaterally and postero-laterally, respectively. The free distal portions of mesofurcal arms expand into very thin blades that almost join, but do not completely fuse to the mesopleural ridge. The free distal portions of metafurcal arms, in its turn, expand dorsally and fuse to the metapleural ridge. Before joining the metapleural ridge, they present a dorsolaterally oriented outgrowth, the dorsal metafurcal lamella (Fig. 3, A-B), which is frequently reinforced laterally by a sclerotic bar from which a filamentous apodeme originates.

\section{1. 3. Mesophragma (Figs. 3, C; 11: 1-44)}

The mesophragma of bees (Fig. 3; C) is a large cuticular inflection that forms an arched structure expanded posteriorly inside the cavity of the propodeum. It is connected to the remainder of the mesosoma by its antero-lateral portion, the mesolaterophragma, which joins the mesophragma to the posterior lateral scutellar inflection (Snodgrass, 1910; Michener, 1944). The main body of the sclerite is delimited anteriorly by the anterodorsal margin of mesophragma and posteriorly by the posteroventral margin of mesophragma. The former is normally feebly sclerotized and expanded into a pseudophragmal lobe. The anterodorsal margin can be almost continuous, only 
interrupted medially by the midddorsal notch of mesophragma, or irregular with several reentrances. The posteroventral margin of mesophragma is also expanded and irregularshaped, frequently possessing a deep middventral notch of mesophragma, through which the dorsal vessel crosses the mesosoma to the metasoma (Michener, 1944). The anterior surface of the mesophragma is deeply concave and is the site of attachment for the longitudinal indirect flight muscles. It commonly presents a strongly developed longitudinal ridge, the mesophragmal longitudinal ridge whose extension is variable in different groups (Fig. 11: 1-44). The posterior surface of the mesophragma is strongly convex and more or less accompanies the shape of the propodeum. It presents a pair of lateral ridges, the mesophragmal arched ridges, which varies in orientation and development. A pair of longitudinal or oblique impressions on the dorso-lateral areas of the posterior surface of mesophragma is also present, the mesophragmal pockets.

\section{2. Character list}

The comparative study of the mesosoma resulted in the construction of a total of 28 characters -13 from prosternum, 5 from propleuron, 6 from mesofurca/metafurca, and 4 from mesophragma - of which 26 were coded as binary and 2 as multistate. The following list presents the characters constructed. The matrix of character state distributions is given on Table 3.

\section{Propectus}

\section{Prosternum}

1. Anterior process of the prosternum (Fig. 1, A): (0) not expanded (Fig. 4: 1-33, 36-44); (1) expanded laterally (Fig. 4: 34-35).

The condition represented by state 1 is characteristic for Bombus spp. Although the anterior process of S. depilis (Fig. 4: 41), Ap. dorsata (Fig. 4: 43) and Ap. mellifera (Fig. 4: 44) are also somehow expanded, they are more triangular and do not form a broad ogivoid expansion as in Bombus, thus the condition presented seems to be acquired independently. They are all coded as 0 .

2. Lateral processes of the prosternum (Fig. 1, A): (0) weakly produced or nearly absent (Fig. 4: 1-35); (1) strongly produced (Fig. 4: 36-44).

Michener (1944) considered the lateral processes of the species of Bombus he studied to be particularly long. Here, if his view was adopted, the processes should be 
coded as strongly produced (state 1). However, it is not completely clear if the lateral processes of Bombus spp. are produced as a result of the expansion of the anterior process/basisternum as a whole or if it is an independent transformation. Therefore, adopting a conservative view, this character was coded as 0 for B. pauloensis (Fig. 4: 34) and B. ternarius (Fig. 4: 35).

3. Profurcal pit (Fig. 1, A): (0) present (Fig. 4: 1-35); (1) absent (Fig. 4: 36-44).

This character was also proposed by Prentice (1991). It seems that the presence of an at least shallow and incipient profurcal pit (state 0) is a general feature of almost all observed taxa. The absence of the profurcal pit (state 1) is found only in the representatives of Apis spp. (Fig. 4: 43-44) and all meliponine taxa (Fig. 4: 36-42).

4. Excavation of the profurcal pit (Fig. 1, A): (0) deep, mainly delimited by the basisternal carina (Fig. 4: 1-29); (1) shallow, sometimes with only an incipient basisternal carina restricted to its posterior portion (Fig. 4: 30-35).

State 0 , is found in all non-corbiculate taxa, whereas state 1 is found only in the observed species of Bombus spp. (Fig. 4: 34-35) and all the euglossine representatives (Fig. 4: 30-33). In Bombina, the profurcal pit is very shallow, but a complete basisternal carina is present. In Euglossina, especially in Euf. violacea (Fig. 4: 31), Eul. nigrita (Fig. 4: 32), and in Eug. melanotricha (Fig. 4: 33), the basisternal carina is restricted to the posterior portion of the shallow profurcal pit.

5. Posterior extensions of the basisternal carina creating two median carinas on the surface of the furcasternum (Fig. 1, A): (0) absent (Fig. 4: 1-5, 7-8, 10, 17-44); (1) present (Fig. 4: $6,9,11-16)$.

The presence of these median carinas on the surface of the furcasternum (state 1) is a condition found in all the colletid representatives (Fig. 4: 11-16) and two other andrenid (Fig. 4: 6) and halictid taxa (Fig. 4: 9). In Acanthopus sp. (Fig. 4: 20), the surface of the furcasternum is very reduced by the greatly expansion of the profurcal pit. This character was considered inapplicable for this taxon. In Meliponina and Apina, although the profurcal pit and basisternal carina are absent, the furcasternum is well-developed and there is no indication of median carinas on its surface. Thus, these taxa are coded as 0 .

6. Connection of the basisternum to the furcasternum (Fig. 1, A): (0) broad (Fig. 4: 1-35); (1) strongly constricted (Fig. 4: 36-44). 
Prentice (1991) also proposed this character in his phylogenetic analysis of corbiculate bees. State 1 is found only in the species of Apis spp. (Fig. 4: 43-44) and Meliponina (Fig. 4: 36-42).

7. Surface of the anterior process of the prosternum (Fig. 1, A): (0) smooth (Fig. 4: 1-29, 34-44); (1) with a series of well-demarcated transversal lines (Fig. 4: 30-33).

Well-demarcated lines (state 1) are found only in Euglossina, although weak lines are frequently seen in some other taxa as well. They appear to represent areas of feebly sclerotized cuticle associated to the dorsal bending of the anterior process in these taxa.

8. Surface of the basisternum (Fig. 1, A): (0) with bristles (Fig. 4: 1-35, 42); (1) smooth (Fig. 4: 36-41, 43-44).

Prentice (1991) considered the presence or absence of bristles on the surface of the prosternum as a single character. Here, it is broken into two separate characters (chars. 9 and 10), considering the surface of both, the basisternum and the furcasternum. They seem to be independent regions, once there are taxa which exhibit bristles in one area but not in the other, and vice-versa. A smooth basisternum, with no bristles (state 1) is found only in Apis spp. (Fig. 4: 43-44) and almost all species pertaining to the Meliponina (Fig. 4: 36-41), with exception of Trigona hyalinata (Fig. 4: 42), which presents bristles only on a small median portion of the basisternum, and therefore was coded as state 0 .

9. Surface of the furcasternum (Fig. 1, A): (0) with strong bristles (Fig. 4: 4-16, 18-19, 21, 25-29); (1) smooth (Fig. 4: 1-3, 17, 20, 22-24, 30-44).

The absence of strong bristles on the surface of furcasternum (state 1) is observed in all the species of corbiculate bees (Fig. 4: 30-44), a few other Apidae (Fig. 4: 17, 20, $22-$ 24), and all the Mellitidae studied (Fig. 4: 1-3). All the remainder taxa present strong bristles on the surface of the furcasternum (state 0 ), which in some oil-collecting bees such as the Cetridini - are very robust and abundant. In Col. inaequalis (Fig. 4: 14) and Lon. robertsi (Fig. 4: 16) there are bristles on the surface of furcasternum but they were omitted for clarity. As for character 5, this character was considered inapplicable for Acanthopus sp. (Fig. 4: 20).

10. Median profurcal lamella: (0) forming a single blade (Fig. 5: 1-30, 34-44); (1) forming a double blade (Fig. 5: 31-33).

State 0 represent the complete fusion of the inner profurcal lamellas. When the arms are fused, but the dorsal margins of the inner lamellas are not completely united, they 
remain as two separate blades (state 1) above the profurcal bridge. This condition is found in most of the Euglossina representatives.

11. Internal cuticular expansions running along the posterodorsal profurcal apodeme: (0) absent (Fig. 5: 1-30, 34-44); (1) present (Fig. 5: 31-33).

The cuticular expansions, when present (state 1), run along the margins of the inner and outer profurcal lamellas converging at the posterodorsal profurcal apodeme. They form a thin sclerotic sheet that expands to the internal surface of each profurcal arm and probably provide extra surface for the attachment of muscles inserted onto the arms. State 1 is found in most of the Euglossina.

12. Pair of twisted lamellas running along the posterior vertical plate of the profurcal arms:

(0) absent (Fig. 4: 4-44); (1) present (Fig. 4: 1-3).

State 1 is found only in the Mellitidae.

13. Ventral profurcal lamella (Fig. 1, C): (0) strongly produced (Figs. 4-5: 1-16, 18-19, 21 24, 26-32, 34-35); (1) weakly produced (Figs. 4-5: 17, 20, 25, 33, 36-44).

State 0 is found in almost all non-corbiculate taxa, with exception of $N$. profuga (Fig. 4: 17), Acanthopus sp. (Fig. 4: 20), and Tetrapedia maura (Fig. 4: 25). The ventral profurcal lamella in Bombina (Fig. 4: 34-35) and most Euglossina (Fig. 4: 30-32) is not exactly strongly produced, but it is well-developed if compared to the other Apini, thus they are coded 0 as well.

\section{Propleuron}

14. Tilt angle of the propleural arm (Fig. 2, A): (0) low (Fig. 6: 3-4, 11-17, 29-33, 43); (1) moderate (Fig. 6: 1, 2, 5, 7, 10, 18-22, 24, 26, 28, 34, 35, 44); (2) high (Fig. 6: 6, 8-9, 23, 27, 36-42).

The degree of tilt was estimated measuring the angle between the propleural arm and the longitudinal axis of the dorsal propleural margin. The classes of values considered for each state were as follows: less than or equal to 115 degrees (low); between 116 and 125 degrees (moderate); more than 125 degrees (high). This character was treated as ordered.

High tilt angles (state 2) are especially evident in a few very small meliponine specimens, such as Trigonisca nataliae (Fig. 6: 38) and Tet. angustula (Fig. 6: 40).

15. Sheet of the propleural arm (Fig. 2, A): (0) slender (Fig. 6: 1-5, 7-9, 11-19, 21-26, 3444); (1) broad (Fig. 6: 6, 10, 20, 27-33). 
The width of the sheets was roughly estimated measuring the ratio of the length to the width of the entire propleural arm. The classes of values considered for each state were as follows: more than or equal to 2.5 (slender sheet); less than or equal to 2.0 (broad sheet).

The state 1 is particularly evident in some specimens pertaining to the Euglossina, such as Euf. violacea (Fig. 6: 31), Eul. nigrita (Fig. 6: 32), and Eug. melanotricha (Fig. 6: $33)$.

16. Propleural carina: (0) strongly developed into a lamella (Figs. 6-7: 1-3, 7-9, 11, 13-14, 16); (1) not developed into a lamella (Figs. 6-7: 4, 10, 12, 15, 17-44).

When the lamella is developed (state 0), a profound groove on the ventral propleural area is usually also present following in parallel the former; otherwise the groove is absent, but an at least weak carina is always present (state 1). Roig-Alsina \& Michener (1993) also utilized the propleural carina in their phylogenetic analysis of longtongued bees. However, they proposed the absence or presence as states, whereas here, it is assumed that an at least weak carina is always present, since a sharp line separating the ventral and lateral propleural areas was observed in all examined taxa. The propleural carina is not developed into a lamella (state 1) in Megachilidae (Figs. 6-7: 17-19), Apidae (Figs. 6-7: 20-44) and a few other taxa.

17. Anterior margin of the ventral propleural area (Fig. 2, C): (0) straight or only slightly emarginated (Fig. 7: 1-33, 36-44); (1) strongly emarginated (Fig. 7: 34-35).

Strongly emarginated anterior margins (state 2) are observed only in Bombina (Fig. 7: 34-35).

18. Sclerotic ring on the ventral propleural area (Fig. 2, C): (0) absent (Fig. 7: 1-2, 4, 6-9, 12-13, 15, 17-20, 23-26, 30, 34-44); (1) present (Fig. 7: 3, 5, 11, 14, 16, 22, 27-29, 31-33).

The sclerotic ring is found (state 1) in a few large specimens of Apidae, such as many representatives of the Euglossina (Fig. 7: 31-33), Centridini (Fig. 7: 27-29), Anthophora sp. (Fig. 7: 20), Melissodes sp. (Fig. 7: 22), and some other non-apid taxa.

\section{Mesofurca/Metafurca}

19. Horizontal plate of the mesofurca (Fig. 3, A): (0) broad, sometimes constricted at its posterior third, but with an expanded anterior portion (Fig. 9: 1-13, 17-44); (1) narrow for the most part of its length (Fig. 9: 14-16).

State 1 is found only in some Colletidae (Fig. 9: 14-16). 
20. Anterior margin of the mesofurcal bridge (Fig. 3, A): (0) entire (Fig. 9: 1-29, 34-44); (1) with a somewhat deep median emargination (Fig. 9: 30-33).

State 1 is found only in the euglossine clade. All other non-euglossids present an entire anterior margin, with no evident emargination (state 0 ). A completely divided bridge in Eug. melanotricha was considered a byproduct of a very deep median emargination, once in the male specimen of this same species and in another observed species of the same genus, the bridge is complete. Thus, Eug. melanotricha was also coded as 1 for this character.

21. Pair of median impressions on the dorsal surface of the mesofurcal bridge sometimes delimiting a broad triangular area (Fig. 3, A): (0) absent (Fig. 9: 20, 37-38, 40-41, 43-44); (1) present (Fig. 9: $\mathbf{X}$ ).

State 0 is found only in Acanthopus sp. (Figs. 9: 20), a few Meliponina (Figs. 9: 3738, 40-41), and Apis spp. (Figs. 9-10: 43-44). In some groups as Das. hirtipes (Figs. 9: 1), Ag. tyleri (Figs. 9: 9), Melissodes sp. (Figs. 9: 22) and a few others, these impressions are very small and restricted to the anterior most portion of the mesofurcal bridge, but they are present, thus these taxa are coded as 1. This character was considered inapplicable for Eug. melanotricha (Figs. 9: 33), once its mesofurcal bridge is incomplete medially.

22. Round apodemes at the antero-lateral corners of the mesofurcal bridge (Fig. 3, A): (0) absent (Fig. 9: 20, 37-38, 40-41, 43-44); (1) present (Fig. 9: 1-19, 21-32, 34-36, 39, 42).

The round apodemes are present (state 1) in the majority of the Apidae, with exception of Meliponina (Figs. 9-10: 36-42), Apina (Figs. 9-10: 43-44), and a few other species. (Figs. 9-10: 20-21, 26, 33). They are especially developed in Centridini (Figs. 9: 27-29) and most Euglossina (Figs. 9: 30-32). In other families, this condition was found only in Ag. tyleri (Figs. 9-10: 9), Ct. nigricans (Figs. 9-10: 10), Li. huberi (Figs. 9-10: 18), and An. manicatum (Figs. 9-10: 19). In Bombus spp. (Figs. 9-10: 34-35) the apodemes are considerably small and dislocated laterally to the free basal portions of mesofurcal arms, but they are also present.

23. Dorsal metafurcal lamella (Fig. 3, B): (0) not folded (Fig. 11: 1-10, 17-44); (1) folded anteriorly, forming a conspicuous anterior projection (Fig. 9: 11-16).

State 1 is found only in Colletidae (Fig. 9: 11-16).

24. Filamentous apodeme on the dorsal metafurcal lamella (Fig. 3, B): (0) terminal to the sclerotic bar (Fig. 10: 1-5, 7-22, 25, 30, 34-35, 37, 40-41, 43-44); (1) dislocated laterally in relation to the sclerotic bar (Fig. 10: 6, 23-24, 26-29, 31-33, 36, 38-39, 42). 
When the apodeme is very reduced as found in Meg. binghami (Figs. 10: 2), Melitta haemorrhoidalis (Figs. 10: 3), Prota. verbesinae (Figs. 10: 5) and some other taxa, the corresponding muscular attachment is presumed to be at the terminal portion of the dorsal metafurcal lamella, thus these taxa were coded as 0 .

\section{Mesophragma}

25. Mesophragmal longitudinal ridge (Fig. 3, C): (0) present (Fig. 11: 1-23, 26-43); (1) absent (Fig. 11: 24-25).

This ridge is completely absent (state 1) in Tapinotaspoides sp. (Fig. 11: 24) and Tetrapedia maura (Fig. 11: 25). In Dac. staudingeri (Fig. 11: 36), Ap. dorsata (Fig. 11: 43) and Ap. mellifera (Fig. 11: 44) a weak ridge or impression is present on the antero-dorsal portion of the mesophragma. They are regarded as a remnant of the mesophragmal longitudinal ridge, thus these taxa are coded as 0 for this character.

26. Mesophragmal arched ridges (Fig. 3, C): (0) more or less parallel to the anteroposterior axis of the mesophragma (parallel ridges) (Fig. 11: 1-19, 22-26); (1) obliquely oriented in relation to the antero-posterior axis of the mesophragma (oblique ridges) (Fig. 11: 20-21, 27-33); (2) centralized and forming two broad arches on the median area of the mesophragma (arched ridges) (Fig. 11: 34-44).

Prentice (1991) has already recognized the arched condition of these ridges (state 2) as a probable derived character state uniting Apina, Bombina and Meliponina (Fig. 11: 3433). Strongly raised oblique ridges (state 1) are found in Acanthpus sp. (Fig. 11: 20), Anthophora sp. (Fig. 11: 21) and Centridini (Fig. 11: 27-29). More or less parallel-oriented ridges (state 0 ), sometimes slightly arched at their extremities, are present on the posterodorsal surface of the mesophragma of most non-corbiculate taxa (Fig. 11: 1-19, 22-26). This character was treated as non-ordered.

27. Anterodorsal margin of mesophragma (Fig. 3, C): (0) well-sclerotized, with a more or less regular contour only interrupted medially by the midddorsal notch of mesophragma (Fig. 11: 1-18, 20, 22-26, 33-44); (1) feebly sclerotized, with an irregular contour demarcated by several reentrances (Fig. 11: 19, 21, 27-32).

A feebly sclerotized and irregular anterodorsal margin of mesophragma (state 1) is found in An. manicatum (Fig. 11: 19), Anthophora sp. (Fig. 11: 21), Centridini (Fig. 11: 27-29), and most Euglossina (Fig. 11: 28-32). The antero-dorsal portion of the mesophragma is conspicuously expanded in these taxa and seems to be developed as 
pseudophragmal lobes, as occurs in some other hymenopteran groups (Melo, 1999; Vilhelmsen et al., 2010), although the homology of these structures throughout Hymenoptera is certainly questionable.

28. Posteroventral margin of mesophragma (Fig. 3, C): (0) fully sclerotized (Fig. 11: 1-18, 22-26); (1) at least partly membranous (Fig. 11: 19-21, 27-29).

Feebly sclerotized posteroventral margins (sate 1) are present in An. manicatum (Fig. 11: 19), Acanthopus sp. (Fig. 11: 20), Anthophora sp. (Fig. 11: 21), and Centridini (Fig. 11: 27-29).

\section{3. Comparative morphology and phylogenetic significance}

The following discussions will be founded on the optimizations of character state changes from mesosoma onto the phylogenetic hypothesis for bees presented by Cardinal \& Danforth (2013) (Fig. 12), with some modifications based on the works of Ramírez et al. (2010), Rasmussen \& Cameron (2010), and the review of Danforth et al. (2013). One thing that becomes clear at first glance when looking to the Figure 12 is that the different portions of mesosoma - hereafter morphological complexes - exhibit distinct phylogenetic signal thus will be discussed separately. The two main constituents of the morphological complex of the propectus, the prosternum and propleuron, were quite distinct in their phylogenetic significance, and then will be discussed individually as well, despite their close association.

\section{3. 1. Morphological complex of the propectus}

\section{Prosternum}

The morphological unity of the prosternum comprises an elaborate structure composed by several portions (e.g., basisternum, furcasternum, profurca) that seems to be multivariate, thus implying some difficulty to distinguish when different areas of the structure change alone or if the shape as a whole modifies altogether. The latter appears to be true for some areas of the anterior portion of the prosternum (e.g., basisternum) and for the general morphology of the profurcal arms. Considering this, the variation found in the prosterna of bees will be split into broader morphotypes, based on the shape of entire areas and clusters of traits. Then, specific changes and their phylogenetic significance will be particularly addressed for each morphotype defined. The main variations were found in the shape of the basisternum, including the anterior and lateral processes; in the depth and 
development of the profurcal pit and basisternal carina; in the pubescence on the exposed surfaces (i.e., basisternum and furcasternum), and in the development of the lamellas of the profurcal arms. Character state changes of the prosternum are depicted as circles on Figure 12.

Two broader morphotypes can be defined for the prosterna of bees. One of them is that exhibited by most non-corbiculate taxa (Fig. 4-5: 1-29). These taxa commonly present a moderately expanded basisternum, with a conspicuous anterior process, weakly to moderately expanded lateral processes, and a complete basisternal carina. The majority of the taxa pertaining to this morphotype, with exception of Melittidae (Fig. 5: 1-3), Anthophora sp. (Fig. 5: 21), Man. postica (Fig. 5: 26), and Centridini (Fig. 5: 27-29) also present completely fused profurcal arms. Furthermore, all the taxa pertaining to this morphotype present a deep profurcal pit and, the majority of them, pubescence on the surfaces of the basisternum/furcasternum. The other morphotype is that of corbiculate bees, in which the basisternum is considerably expanded due to the great development of the anterior/or lateral processes (e.g., Apina, Bombina, and Meliponina) and relative posterior displacement of the basisternal carina (e.g., Euglossina), which loses its anterior portions and becomes incomplete. The condition of the profurcal arms is variable in this morphotype; Apina (Fig. 5: 43-44) and Euglossina (Fig. 5: 30-33) present completely fused profurcal arms, whereas Bombina (Fig. 5: 34-35) and most Meliponina (Fig. 5: 37, 40-42) present separate arms. Also, the profurcal pit is very shallow or absent (char. 4:1) and the furcasternum smooth (char. 9:1) in all corbiculate bees. The former will be referred as the A-morphotype and the latter as B-morphotype.

In the A-morphotype, the posterior extensions of the basisternal carina creating two median carinas on the surface of the furcasternum (char. 5:1) support the monophyly of the Colletidae (Fig. 4: 11-16). This condition has appeared at least two other times, in Proto. glogiosa (Fig. 4: 6) and Ag. tyleri (Fig. 4: 9). The condition found in the latter species, however, is not exactly identical to that found in the colletid taxa. In this species, the connection of the two median carinas with the basisternal carina is greatly lost and they are displaced posteriorly on the surface of the furcasternum. A smooth furcasternum (char. 9:1) has evolved two times in this morphotype, one in N. profuga (Fig. 4: 17) and other in the clade including Melissodes sp., Diadasina sp., and Tapinotaspoides sp. (Fig. 4: 22-24). Weakly produced ventral profurcal lamellas (char. 13:1) is an autapomorfic character state for Acanthopus sp. (Fig. 4: 20) and Tetrapedia maura (Fig. 4: 26). The condition presented 
by the former, however, is certainly quite different, since the whole prosternum in this taxon is aberrantly modified, with a greatly expanded profurcal pit occupying much of the surface of the prosternum, thus leaving a much reduced furcasternum in addition to a nearly absent ventral profurcal lamella. A much similar morphology was depicted in one illustration of the prosternum of Mesonychium jenseni (Roig-Alsina \& Michener, 1993; fig. 21), also a member of the parasitic apid tribe Ericrocidini. Thus, this aberrant morphology seems to be characteristic for this tribe. Another distinctive derived character state found in a few taxa pertaining to the A-morphotype is a pair of twisted lamellas running along the posterior vertical plate of the profurcal arms (char. 12:1). These lamellas are present only in Melittidae (Fig. 4: 1-3), although not shown in the Figure 12. The optimization for this character is ambiguous, since more external taxa were not observed in this study, but it seems to be unique to these taxa.

The character state changes in the B-morphotype were evaluated under two contrasting scenarios (Figure 12). In scenario I, a shallow or absent profurcal pit (char. 4:1) and the absence of pubescence on the surface of furcasternum (char. 9:1) are synapomorphies of the corbiculate clade. Under this scenario (I), the strongly produced lateral processes of the prosternum (char. 2:1), complete absence of an external indication of the profurcal pit (char. 3:1), strong constriction between the basisternum and the furcasternum (char. 6:1), absence of pubescence on the surface of basisternum (char. 8:1), and much reduced ventral profurcal lamellas (char. 13:1) have evolved twice in Meliponina (Fig. 4-5: 36-42) and Apina (Fig. 4-5: 43-44). In the alternative scenario (II), all these character state changes have appeared once in the ancestral species of the ApinaMeliponina clade. While the smooth condition of the basisternum was reversed in Trigona hyalinata (char. 8:0) and the reduced ventral profurcal lamellas (char. 13:1) were also present in Acanthopus sp. (Fig. 4: 20), Tetrapedia maura (Fig. 4: 26), and Eug. melanotricha (Fig. 4: 33), the well-developed lateral processes of the prosternum, complete absence of a profurcal pit and strongly constricted connection of the basisternum to the furcasternum are conditions not seen in any other bee taxa, thus it is difficult to accept that they arose independently twice in light of the dataset presented here.

In both scenarios, the broadly expanded anterior process of the prosternum (char. $1: 1$ ) is synapomorphic for Bombina (Fig. 4: 34-35); the surface of the anterior process of the prosternum with well-demarcated folding lines (char. 7:1) is synapomorphic for Euglossina (Fig. 4: 30-33); the median profurcal lamella with a double blade (char. 10:1) 
and the cuticular expansions running along the posterodorsal profurcal apodeme (char. 11:1) are putative synapomorphies for Euf. violacea, Eul. nigrita, and Eug. melanotricha (Fig. 5: 31-33).

Prentice (1991) was the first to recognize the importance of the prosternum as a rich source of phylogenetic information to the study of the relationships of corbiculate bees. Roig-Alsina \& Michener (1993) and Melo (1999) also acknowledge its importance and have took advantage of characters derived from the prosternal structure to use in their phylogenetic analyses of long-tongued bees and apoid wasps, respectively. Prentice (1991) reunited Bombina, Meliponina and Apina based on the presence of a much enlarged basisternum and this is considered here to be one of the main defining characters for most representatives of the A-morphotype. However, it must be acknowledge that the enlargement of the basisternum in Bombina (Fig. 4: 34-35) seems to be the main result of the expansion of the anterior process, whereas in Apina (Fig. 4: 43-44) and Meliponina (Fig. 4: 36-42), there is also considerable extension of this process, but the lateral ones, are the main responsible for the enlargement of the whole basisternum. It is also evident that in Bombina the expansion of the basisternum has occurred independently of the condition presented by the furcasternum. In Apina and Meliponina the enlargement of the basisternum was accompanied by the concomitant reduction of the furcasternum. If the expansion of the basisternum in these three taxa and the reduction of the furcasternum in Apina and Meliponina are homologous, or if they evolved independently, are matters for further discussions. In light of this, the inclusion of information from fossil taxa can be very helpful, since it would elucidate the changes that have occurred in the course of evolution of the different morphological complexes in corbiculate bees (Engel, 2000a, b). For this purpose, it would be interesting to use micro-CT scanning on amber preserved corbiculate specimens as it was done for a parasitoid wasp in the recent work of Lars \& Zimmerman (2014) and a social bee in Greco et al. (2011).

Another remarkable portion of the prosternum is the anterior profurcal apodeme (Figs. 1, B; 5: 1-44). These apodemes are rather simple structures, but seems to exhibit much variability in shape, orientation and rotation amongst different taxa and would be useful for phylogenetic studies. Some of the variation found, however, seems to be partly related to the overall size of the specimens, as large specimens normally present broadened apodemes, while the smaller ones present narrowed apodemes. It is also important to consider that these processes, especially their anterior-most portions, are weakly 
sclerotized. This implies some difficulties to evaluate accurately the true nature and shape of the connections of them with the cervical apodemes of the propleuron. Thus, they were just represented with dashed lines on the plates presented (Fig. 5: 1-44) and no characters derived from them have been proposed for this study.

\section{Propleuron}

The general morphology of the propleuron is much less variable than that of the prosternum and the character state changes exhibited much more plasticity. The main variations were found in the structure of the propleural arm; the development of the propleural carina; the margins and sclerotic modifications of the ventral propleural area, and the shape of the cervical apodeme. Character state changes of the propleuron are depicted as squares on Figure 12. Considering the whole organization of the propleuron, two morphotypes can be roughly defined for this sclerite as it was done for the prosternum. One morphotype comprise those propleura with a somewhat slender propleural arm, whose tilt angle usually is between 115 and 125 degrees; more or less straight median and posterior margins of the ventral propleural area, and frequently a propleural carina welldeveloped into a lamella. This morphotype includes the majority of the propleura from the representatives of Stenotritidae, Colletidae, Halictidae, and Andrenidae (Figs. 6-7: 1-16). The other morphotype includes the propleura of most Megachilidae and Apidae (Fig. 6-7: 17-44), whose propleural arm is variable in terms of the development of its sheet and tilt angle; the anterior, median, and posterior margins of the ventral propleural area are commonly more sinuous; and without a propleural carina developed into a lamella. The former will be referred as $\boldsymbol{C}$-morphotype and the latter as $\boldsymbol{D}$-morphotype.

In the C-morphotype, the tilt angle of the propleural arm in relation to the longitudinal axis of the dorsal propleural margin (char. 14), although between 115 and 125 degrees in most taxa (state 1), have changed many times to states 0 (less than 115 degrees) and 2 (more than 125 degrees). Low tilt angles (char. 14:0) are found in Melitta haemorrhoidalis (Fig. 6: 3), Colletidae (Fig. 6: 11-16), and O. inquirienda (Fig. 6: 4). High tilt angles (char. 14:2), in its turn, are found in the clade constituted by Die. triangulifera and Ag. tyleri (Fig. 6: 8-9), and also in Proto. gloriosa (Fig. 6: 6). The development of a broad sheet of the propleural arm (char. 15:1) has appeared twice in Ct. nigricans (Fig. 6: 10) and Proto. gloriosa (Fig. 6: 10). The reduction of the propleural carina (char. 15:1) occurred convergently in Ct. nigricans (Figs. 6-7: 10), Trichocolletes sp. (Figs. 6-7: 15), 
My. fallax (Figs. 6-7: 12), and O. inquirienda (Figs. 6-7: 4). Despite their plasticity, the variation found in some of these characters (chars. 14-16) seems to be informative for particular instances and have recovered the monophyly of some groups as Colletidae, Die. triangulifera $+A g$. tyleri, and other groups in the long-tongued clade. The complete exclusion of them from this dataset would not be justified only by the occurrence of homoplasy, since if carefully interpreted, they can help to delimit some groups and enable the better understanding of the evolution of complex morphological changes in a broader context for bees.

The D-morphotype is essentially defined by the reduction of the propleural carina, which is not developed as a strong lamella (char. 16:1). This condition, in spite of have already appeared four times in the $\boldsymbol{C}$-morphotype, seems to be consistent with the monophyly of the long-tongued bees (Megachilidae + Apidae), since all the representatives of this groups have reduced carinas. The enlarged sheet of the propleural arm (char. 15:1) and the presence of the sclerotic ring on the ventral propleural area (char. 18:1) support Centridini as monophyletic. Focusing on the relationships of the Apini clade, under both scenarios (I and II), the low tilt angle of the propleural arm (char. 14:0) is a derived character state change for Euglossina (Fig. 6: 30-33) whereas a high tilt angle (char. 14:2) is synapomorphic for Meliponina (Fig. 6: 36-42). Also, the strongly emarginated anterior margin of the ventral propleural area (char. 17:1) is an uncontroverted character state change for the Bombina clade (Fig. 7: 34-35). The presence of the sclerotic ring (char. 18:1), although also present in Centridini (Fig. 7: 27-29), a few other Apidae (Fig. 7: 2122), and many representatives of the C-morphotype, reunites Euf. violacea, Eul. nigrita, and Eug. melanotricha (Fig. 7: 31-33). The sole difference between scenarios I and II is that in the former the condition of a broad sheet of the propleural arm (char. 15:1) is also a derived character state change for Euglossina.

Perhaps, one of the most variable portions of the composite sclerite of the propleuron - which offered much difficulty to define possible homologous states - is the cervical apodeme (Fig. 8: 1-44). The main structure of this morphological unity is rather simple, but the shape of its sheet is quite different in distinct groups, providing considerable complexity to the proper understanding of its connection to muscles present in different portions of the prothorax (see Vilhelmsen, 2000b for more details on the muscular connections in the prothorax in the ground plan of the Hymenoptera). The cranial condyle of propleuron exhibits much less variation than the shape of the sheet of the 
cervical apodeme, but even so, it was hard to encompass the variation observed into discrete states. In some species it has a more rounded shape and is not too projected anteriorly, as it occurs in Lon. robertsi (Fig. 8: 16), Acanthopus sp. (Fig. 8: 20), and Tapinotaspoides sp. (Fig. 8: 24). In others it is also rounded but more protruded, as in Ag. tyleri (Fig. 8: 9), Das. hirtipes (Fig. 8: 1), and N. profuga (Fig. 8: 17). In other species, the condyle is slightly angulate and projected, and in Trichocolletes sp. (Fig. 8: 15), Trigonisca nataliae (Fig. 8: 38) and Tetragonisca angustula (Fig. 8: 40) it is strong angulated. In Euf. violacea (Fig. 8: 31), Ap. dorsata (Fig. 8: 43) and Ap. mellifera (Fig. 8: 44) there is a sharp pointed projection on its dorsal surface.

The structure of the cervical apodeme is also much variable and it was hard to assemble the variation of its shape into discrete states. In a gross way, they can be divided into three broad categories based on the shape of its sheet. The main category includes those cervical apodemes in which the sheet has a more or less quadrangular shape, with somewhat defined dorsal, ventral and posterior margins. This condition is observed in some species as Das. hirtipes (Fig. 8: 1), Melitta haemorrhoidalis (Fig. 8: 3), Anthophora sp. (Fig. 8: 21), and Tetrapedia maura (Fig. 8: 25). A second category comprises those in which the anterodorsal bar of the sheet is strong projected dorso-medially, thus resembling an upright rectangle. This condition occurs in a few species as Centris flavifrons (Fig. 8: 28), Eul. nigrita (Fig. 8: 32), B. ternarius (Fig. 8: 35), Ap. dorsata (Fig. 8: 43), and Ap. mellifera (Fig. 8: 44). A third category would include the cervical apodemes with the posterior bar posteriorly projected, conferring to the sheet an appearance that resembles a horizontal rectangle. This occurs in Acanthopus sp. (Fig. 8: 20), Trigonisca nataliae (Fig. 8: 38), and Trigona hyalinata (Fig. 8: 42). In a few other species, as Eug. melanotricha (Fig. 8: 33), B. pauloensis (Fig. 8: 34), and Melipona quadrifasciata (Fig. 8: 39), the sclerotic sheet is reduced, leaving only the two reinforcement bars. Although described here, the disparities found in the shape of the cranial condyle and cervical apodeme were not used to construct characters, since there were difficulties to establish exact delimitations for the character states.

\section{3. 2. Morphological complex of the mesofurca and metafurca}

As noticed for the propleura of bees, the general morphology of the complex mesofurca/metafurca is subject to a considerably degree of plasticity. The main sources of variation found in this complex were related to the shape of the horizontal plate of the 
mesofurca; general structure of the mesofurcal bridge; and modifications associated to the dorsal metafurcal lamellas. The character state changes for this complex are indicated as triangles on Figure 12. Defining general morphotypes for this complex is rather more difficult than it was for the previous ones, because much of the discrepancies in sizes and shapes can be regarded as continuous variations - especially the lengths of its arms and breadth of the horizontal plate of the mesofurca/mesofurcal bridge. Nonetheless, a more general morphotype can be tentatively defined to include all those complexes with a somewhat broad horizontal plate of the mesofurca, sometimes distinctly constricted posteriorly, and a more or less broad mesofurcal bridge, frequently presenting a pair of median impressions on its dorsal surface. This condition is found in almost all taxa, excluding Apina and most Meliponina. Another attribute that can be also associated to some groups in this morphotype is the presence of two round apodemes at the anterolateral corners of the mesofurcal bridge. This condition is especially evident in Centridini (Fig. 9: 27-29) and Euglossina (Fig. 9: 30-33), although also present in many Apidae with variable degrees of development. A more particular morphotype that can be suggested to include Apina (Fig. 9: 43-44) and most Meliponina (Fig. 9: 37-38, 40-41), comprise those complexes whose mesofurcal bridges are relatively slender, without any median impressions on their dorsal surfaces and totally missing the round apodemes. Despite the descriptive utility, indeed these morphotypes are arbitrary, thus will not be used in the following discussions. In this case, each character and its transformations will be discussed individually.

Considering at first the non-corbiculate taxa, two characters state changes are especially interesting, because each of them have appeared only one time in the tree used for the optimizations. The anterior folding of the dorsal metafurcal lamella (char. 23:1) evolved once in Colletidae (Fig. 9: 11-16) and a distinctively narrowed horizontal plate of the mesofurca (char. 19:1) is an uncontroverted change that supports the monophyly of Co. inaequalis, Trichocolletes sp., and Lon. robertsi (Fig. 9: 14-16). The presence of a pair of median impressions on the dorsal surface of the mesofurcal bridge (char. 21:1) seems to be plesiomorfic for bees as a whole, being lost (state 0) in Acanthopus sp. (Fig. 9: 20). The presence of round apodemes at the antero-lateral corners of the mesofurcal bridge (char. 22:1) is found in Ag. tyleri (Fig. 9: 9), Ct. nigricans (Fig. 9: 10), in the clade composed by Li. huberi (Fig. 9: 18) + An. manicatum (Fig. 9: 19), and in the clade that comprises most Apidae, with exception of Acanthopus sp. + Anthophora sp. The homology of these 
apodemes themselves throughout the studied taxa seems plausible, since they occupy more or less the same position on the mesofurcal bridge in different groups. Their degree of development - from broadly expanded to much reduced or absent - and shape - rounded, tumb-like, elongated - are certainly subject to much plasticity. The laterally displaced filamentous apodeme on the dorsal metafurcal lamella (char. 24:1) supports Centridini (Fig. 10:27-29) and the grouping of Diadasina sp. + Tapinotaspoides sp. For the last two characters discussed - round apodemes and position of the filamentous apodeme on the dorsal metafurcal lamella - despite their utility to define some of the groups mentioned above, caution is necessary to evaluate their phylogenetic significance. They are associated to muscle attachments, thus can be subject to variations due specific mechanical stress patterns in different specimens of the same species. Dissections of more specimens would be desirable to better evaluate some these character states.

As for the corbiculate clade, considering both hypotheses (I and II), the anterior margin of the mesofurcal bridge with a somewhat deep median emargination (char. 20:1) is an uncontroverted character state change supporting Euglossina (Fig. 9: 30-33). Also, the lateral positioning of the filamentous apodeme on the dorsal metafurcal lamella (char. 24:1) is synapomorphic for the remainder Euglossina, excluding Ex. smaragdina. The main disagreement between scenarios I and II is for the optimization of character 22 - presence of the round apodemes. In the first scenario, these apodemes have been missed twice in Apina and Meliponina. In the alternative scenario (II), they were lost just once. As mentioned before, the optimizations for the changes of characters 22 and 24 must be viewed with caution. It is equally plausible to consider that the round apodemes were lost independently in Apina and Meliponina, since many taxa outside Apidae also lack them. Accordingly, the terminal position of the filamentous apodeme (char. 24:0) in Ex. smaragdina could have been changed secondarily from a more lateral position, implying that the lateral positioning of this apodeme is synapomorphic for Euglossina. Another alternative, if one consider only scenario II, is that it could have assumed this lateral position (state 1) once in the ancestral of Centridini + Apini, and then reverted to a more terminal position (state 0) in the exclusive common ancestral of Bombina, Apina and Meliponina, although some taxa in the latter presents lateral positioned apodemes (state 1) as well. 


\section{3. 3. Mesophragma}

The last structure pertaining to the mesosoma herein discussed is the mesophragma. Differently from the last three morphological complexes, the mesophragma constitute a sole sclerite, thus should be viewed as an individual morphological unity. Variations were principally found in the development and shape of the ridges on its posterior surface and in the degree of sclerotization and shape of its anterodorsal and posteroventral margins. All the character state changes of the mesophragma are depicted as stars on Figure 12.

More or less parallel oriented arched ridges on the posterior surface of the mesophragma (char. 26: 1) are a common feature present in all non-Apid taxa (Fig. 11: 119) and in some Apidae as well (Fig. 11: 22-26). In some groups, however, they have changed from this basic pattern and have secondarily assumed various shapes (Fig. 11: 10, 18-19). To proper account for this morphological diversity, separated character states would have been erected for them, but here they are assumed to be secondary modifications from state 0 . Thus, they were arbitrarily coded this way as well, even though the character state changes are probably autapomorphic for the respective taxa. Strongly raised and heavy sclerotic oblique ridges (char. 26:1) are present in the following clades: Acanthopus sp. + Antophora sp., Centridini and Euglossina. These character state changes were not showed in Figure 12 because they were not unambiguously optimized. The condition presented by these taxa, however, are quite similar. One possibility considering scenario II, is that the heavy sclerotic oblique ridges (state 1) evolved once in the common ancestral of Apidae, being reverted to a more parallel condition (state 0) in the clade that includes Tetrapedia maura, Man. postica and closely related groups; and then changed again to a broadly arched condition (state 2) in the exclusive ancestor of Bombina, Apina and Meliponina. Apart of the optimizations for states 0 and 1, the broadly arched ridges on the posterior surface of the mesophragma (char. 26:2) are synapomorphic for the latter clade under scenario II. Considering the alternative scenario (I), a feebly sclerotized anterodorsal margin of mesophragma with an irregular contour (char. 27:1) is synapomorphic for Centridini (Fig. 11: 27-29) and Euglossina (Fig. 11: 30-33), and autapomorphic for An. manicatum (Fig. 11: 19) and Anthophora sp. (Fig. 11: 21). In both scenarios (I and II), a weakly sclerotized posteroventral margin of mesophragma (char. 28:1) have evolved three times, once in the clade Acanthopus sp. + Anthophora sp., another time in Centridini (Fig. 11: 27-29), and again in An. manicatum (Fig. 11: 19). 
Taking into account these four morphological complexes/unities - prosternum, propleuron, mesofurca/metafurca and mesophragma - it is clear that they do not have the same phylogenetic signal, and their significance is not equal throughout the phylogenetic tree of bees. Most of the uncontroverted character state changes of the mesosoma were provided by the prosternum and were concentrated in the corbiculate clade ( 9 changes, considering scenario II). In part, this can be explained because Apini was the main focus of interest of this study, thus it is not surprisingly that most character states were dedicated to solve its relationships. However, it can be also considered that despite the aberrant morphology of the prosternum in Acanthopus sp. - and probably other Ericrocidini - this structure is quite distinct in the corbiculate bees if compared to a more homogeneous configuration in other bee taxa. It can be the consequence of a long branch separating the corbiculate bees from the other Apidae (Engel, 2001a, b) or a rapid diversification of the Apini (Whitfield \& Kjer, 2008), resulting in the observed disparity in the prosternal morphology. On the other hand, the considerable degree of plasticity observed especially in the changes of the propleuron and mesofurca/metafurca, which is indicated by the great number of homoplastic transformations, deserves a careful evaluation. Although part of this plasticity can be associated to allometric consequences of the discrepancies in sizes between distinct specimens and/or differential mechanical stress patterns on the apodemes, some of the character state changes seem to be useful as sources of phylogenetic information in more restricted portions of the tree. Thus, the complete exclusion of these "plastic" characters would result in a considerable loss of information, especially for Colletidae, Centridini, and Euglossina.

Also, it should be mentioned that complex internal structures, despite its phylogenetic relevance as shown here, still are poorly described and used in comparative studies. As it was acknowledged by several authors (Snodgrass, 1942, 1956; Michener, 1944; Prentice, 1991; Roig-Alsina \& Michener, 1993; Melo, 1999) internal skeletal structures provide useful sources of characters for phylogenetic purpose. It this concern, it is important to consider the use of new technologies for phenotypic studies - as MRI, CLSM and micro$\mathrm{CT}$ - and the inclusion of fossil information as a perspective for future studies on corbiculate phylogeny and bee morphology. 


\section{Acknowledgments}

We are very grateful to Denise A. Alves and Sidnei Mateus for providing many of the stingless bees used in this study. We are grateful to Maria Isabel P. Balbi for offering help and suggestions on the preparation of the material examined. The results obtained in this work were supported by the grants \# 2012/22261-8 and \# 2014/10090-0 to D. S. Porto and partially by the grant \# 2011/09477-9 to Eduardo A. B. Almeida, São Paulo Research Foundation (FAPESP). Also, Kevin Nixon is acknowledged for making the Winclada software available. 
Table 1. Material examined. Numbers on the right column make reference to the respective taxa in the figures.

\begin{tabular}{|c|c|c|}
\hline Family & Species & Number \\
\hline \multirow[t]{3}{*}{ MELITTIDAE } & Dasypoda hirtipes (Fabricius, 1793) & 1 \\
\hline & Meganomia binghami (Cockerell, 1909) & 2 \\
\hline & Melitta (Cilissa) haemorrhoidalis (Fabricius, 1775) & 3 \\
\hline \multirow[t]{3}{*}{ ANDRENIDAE } & Orphana inquirenda Vachal, 1909 & 4 \\
\hline & Protandrena verbesinae (Timberlake, 1955) & 5 \\
\hline & Protoxaea gloriosa $($ Fox, 1893$)$ & 6 \\
\hline \multirow[t]{3}{*}{ HALICTIDAE } & Penapis penai Michener, 1965 & 7 \\
\hline & Dieunomia triangulifera $($ Vachal, 1897$)$ & 8 \\
\hline & Agapostemon tyleri Cockerell, 1917 & 9 \\
\hline STENOTRITIDAE & Ctenocolletes nigricans Houston, 1985 & 10 \\
\hline \multirow[t]{6}{*}{ COLLETIDAE } & Paracolletes crassipes Smith, 1853 & 11 \\
\hline & Mydrosoma fallax (Moure, 1953) & 12 \\
\hline & Callomelitta antipodes (Smith, 1853) & 13 \\
\hline & Colletes inaequalis Say, 1837 & 14 \\
\hline & Trichocolletes sp. & 15 \\
\hline & Lonchopria robertsi Michener, 1989 & 16 \\
\hline \multirow[t]{3}{*}{ MEGACHILIDAE } & Neofidelia profuga Moure and Michener, 1955 & 17 \\
\hline & Lithurgus (Lithurgus) huberi Ducke, 1907 & 18 \\
\hline & Anthidium manicatum (Linnaeus, 1758) & 19 \\
\hline Ericrocidini & Acanthopus sp. & 20 \\
\hline Anthophorini & Anthophora sp. & 21 \\
\hline Eucerini & Melissodes sp. & 22 \\
\hline Emphorini & Diadasina sp. & 23 \\
\hline Tapinotaspidini & Tapinotaspoides sp. & 24 \\
\hline Tetrapediini & Tetrapedia maura Cresson, 1878 & 25 \\
\hline Manueliini & Manuelia postica (Spinola, 1851) & 26 \\
\hline \multirow[t]{3}{*}{ Centridini } & Epicharis (Epicharana) flava Friese, 1900 & 27 \\
\hline & Centris (Centris) flavifrons (Fabricius, 1775) & 28 \\
\hline & Centris (Heterocentris) analis (Fabricius, 1804) & 29 \\
\hline \multirow[t]{4}{*}{ Apini: Euglossina } & Exaerete smaragdina (Guérin, 1844) & 30 \\
\hline & Eufriesea violacea (Blanchard, 1840) & 31 \\
\hline & Eulaema (Apeulaema) nigrita Lepeletier, 1841 & 32 \\
\hline & Euglossa (Euglossa) melanotricha Moure, 1967 & 33 \\
\hline \multirow[t]{2}{*}{ Apini: Bombina } & Bombus (Fervidobombus) pauloensis Friese, 1913 & 34 \\
\hline & Bombus (Pyrobombus) ternarius Say, 1837 & 35 \\
\hline
\end{tabular}


Table 1. Continuation.

\begin{tabular}{llr}
\hline Family & Species & Number \\
\hline \multirow{4}{*}{ Apini: Meliponina } & & \\
& & 36 \\
& Dactylurina staudingeri (Gribodo, 1893) & 37 \\
& Lophotrigona canifrons (Smith, 1857) & 38 \\
& Trigonisca (Trigonisca) nataliae (Moure, 1950) & 39 \\
& Melipona (Melipona) quadrifasciata Lepeletier, 1836 & 40 \\
& Tetragonisca angustula (Latreille, 1811) & 41 \\
& Scaptotrigona depilis (Moure, 1942) & 42 \\
& Trigona hyalinata (Lepeletier, 1836) & 43 \\
Apini: Apina & & 44 \\
& Apis dorsata Fabricius, 1793 & \\
\hline & Apis mellifera Linnaeus, 1758 & \\
\hline
\end{tabular}


Table 2. (next page) Morphological terminology used previously by other authors to make reference to some structures of the mesosoma. ${ }^{*}=$ terms not explicitly proposed by the authors in those works, but used in morphological descriptions or figure captions; ${ }^{* \star}=$ changes of terminology or new terms introduced to make correspondence to more general terms already used in other hymenopteran groups. Abbreviations: CDM, C. D. Michener (1944); HAO, Hymenoptera Anatomy Ontology; LV, Lars Vilhelmsen (2000a, b); LV\&al, Vilhelmsen et al. (2010); RA\&M, Roig-Alsina \& Michener; RES, R. E. Snodgrass (1910, 1942, and 1956). 


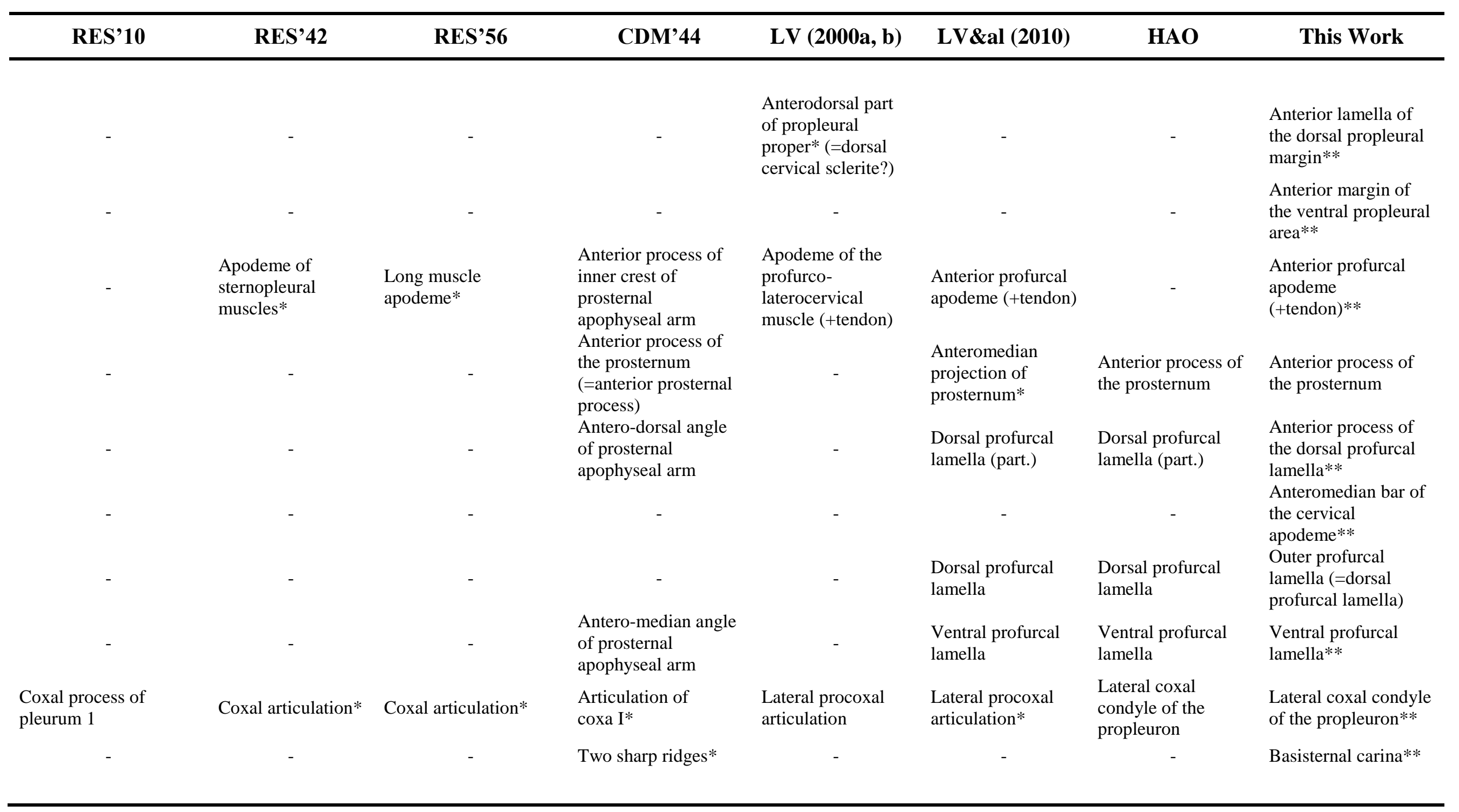


Table 2. Continuation

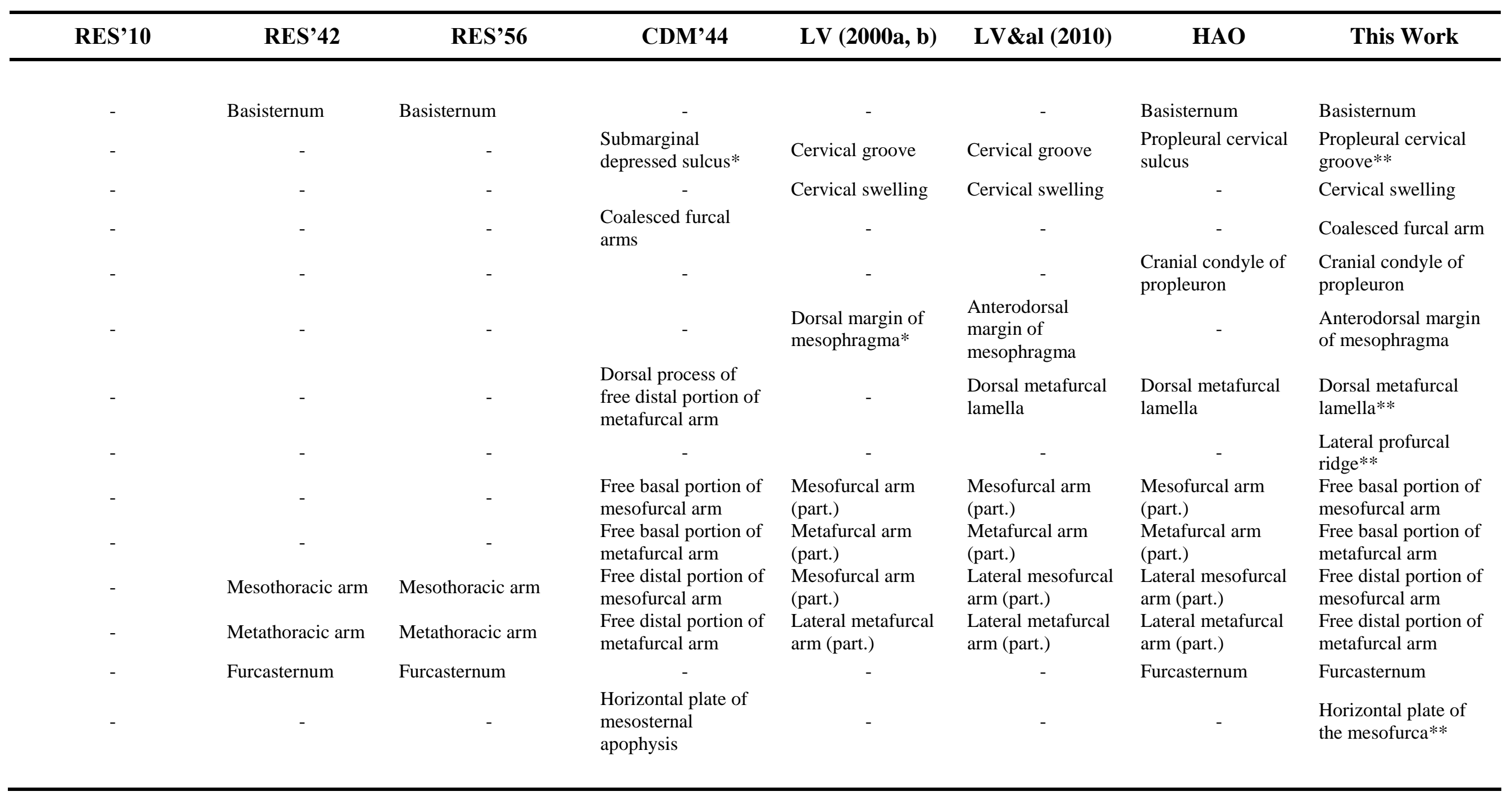


Table 2. Continuation

\begin{tabular}{|c|c|c|c|c|c|c|c|}
\hline RES'10 & RES'42 & RES'56 & CDM'44 & LV (2000a, b) & LV\&al (2010) & HAO & This Work \\
\hline- & - & - & $\begin{array}{l}\text { Horizontal plate of } \\
\text { prosternal apophyses }\end{array}$ & - & - & - & $\begin{array}{l}\text { Horizontal plate of } \\
\text { the profurca** }\end{array}$ \\
\hline- & - & - & $\begin{array}{l}\text { Inner crest of } \\
\text { prosternal } \\
\text { apophyseal arm }\end{array}$ & - & - & $\begin{array}{l}\text { Anterior profurcal } \\
\text { lamella }\end{array}$ & $\begin{array}{l}\text { Inner profurcal } \\
\text { lamella** }\end{array}$ \\
\hline- & - & - & Intercoxal lamella & Paracoxal ridge & Paracoxal ridge & Paracoxal ridge & Paracoxal ridge** \\
\hline- & - & - & - & - & - & - & $\begin{array}{l}\text { Lateral margin of the } \\
\text { prosternum** }\end{array}$ \\
\hline- & - & - & $\begin{array}{l}\text { Lateral portion of } \\
\text { propleuron }\end{array}$ & - & - & $\begin{array}{l}\text { Lateral propleural } \\
\text { area }\end{array}$ & $\begin{array}{l}\text { Lateral propleural } \\
\text { area** }\end{array}$ \\
\hline- & - & - & $\begin{array}{l}\text { Lateral process of the } \\
\text { prosternum }\end{array}$ & - & - & ( & $\begin{array}{l}\text { Lateral process of the } \\
\text { prosternum }\end{array}$ \\
\hline Epimeral arm & Pleural apophysis & Apodemal arm & $\begin{array}{l}\text { Marginal apodeme of } \\
\text { propleuron }\end{array}$ & Propleural arm & Propleural arm & Propleural arm & Propleural arm $* *$ \\
\hline- & - & - & $\begin{array}{l}\text { Median crest of } \\
\text { prosternal apophysis }\end{array}$ & - & - & - & $\begin{array}{l}\text { Median profurcal } \\
\text { lamella** }\end{array}$ \\
\hline- & $\begin{array}{l}\text { Median groove* } \\
\text { (Fig. II, K, f) }\end{array}$ & - & $\begin{array}{l}\text { Median prosternal } \\
\text { groove }\end{array}$ & $\begin{array}{l}\text { Discrimen of } \\
\text { prothorax }\end{array}$ & Prodiscrimen & Prodiscrimen & Prodiscrimen $* *$ \\
\hline- & 年 & - & $\begin{array}{l}\text { Midventral notch of } \\
\text { second phragma* }\end{array}$ & 1 & - & - & $\begin{array}{l}\text { Midventral notch of } \\
\text { mesophragma** }\end{array}$ \\
\hline- & - & - & $x^{2}=0$ & $\begin{array}{l}\text { Opening of the } \\
\text { mesoscutello- } \\
\text { metanotal muscle } \\
\text { tendon* }\end{array}$ & - & - & $\begin{array}{l}\text { Middorsal notch of } \\
\text { mesophragma** }\end{array}$ \\
\hline- & - & - & - & (1) & - & - & $\begin{array}{l}\text { Median margin of the } \\
\text { ventral propleural } \\
\text { area** }\end{array}$ \\
\hline
\end{tabular}


Table 2. Continuation

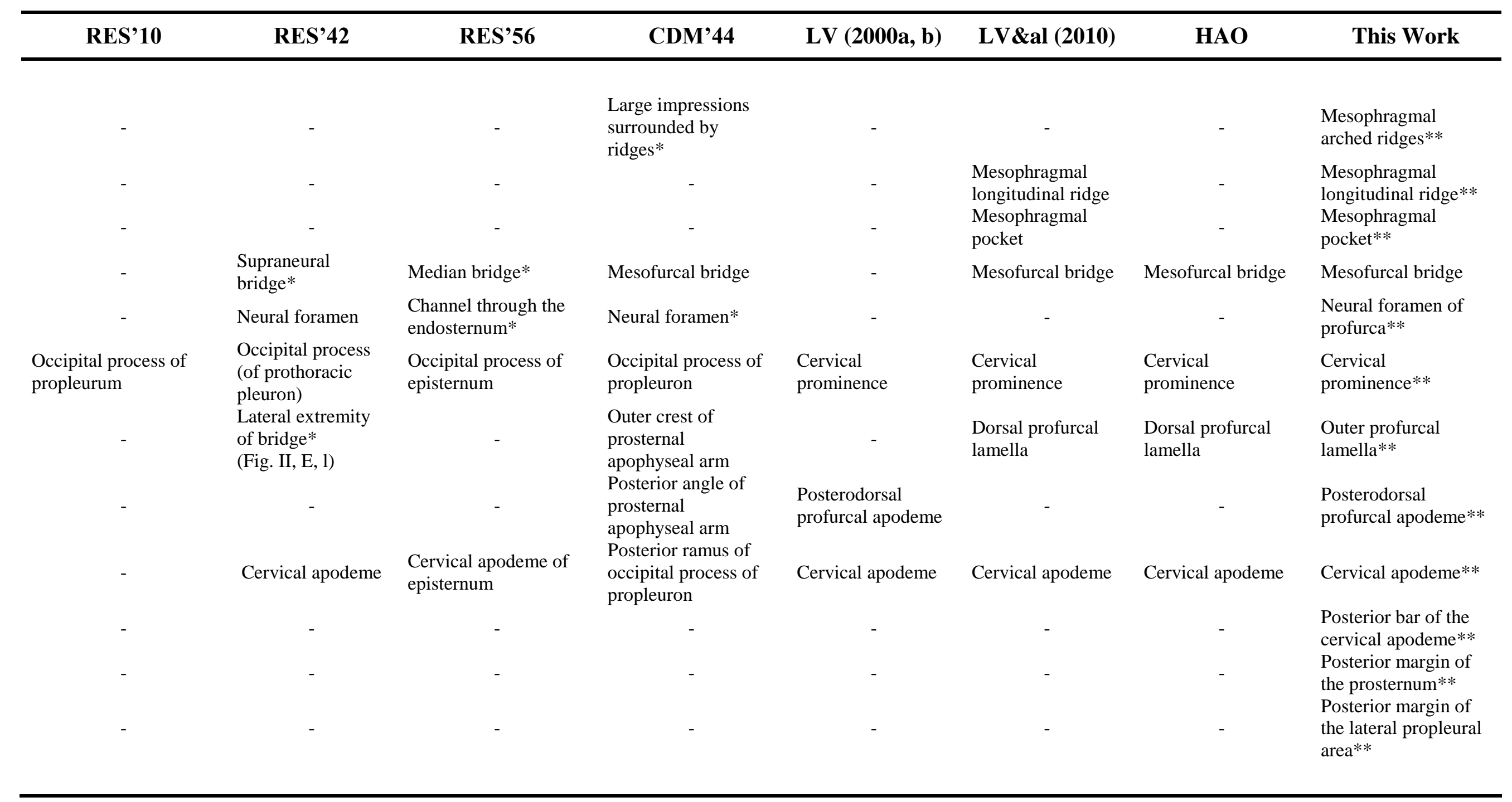


Table 2. Continuation

\begin{tabular}{|c|c|c|c|c|c|c|c|}
\hline RES'10 & RES’42 & RES'56 & CDM'44 & LV (2000a, b) & LV\&al (2010) & HAO & This Work \\
\hline- & - & - & - & - & - & - & $\begin{array}{l}\text { Posterior margin of } \\
\text { the ventral propleural } \\
\text { area** }\end{array}$ \\
\hline- & $\begin{array}{l}\text { Posterior angle of } \\
\text { the horizontal } \\
\text { apodeme* }\end{array}$ & - & - & - & - & - & $\begin{array}{l}\text { Posterior process of } \\
\text { the dorsal propleural } \\
\text { ridge** }\end{array}$ \\
\hline- & $\begin{array}{l}\text { Lateral } \\
\text { thickenings* (Fig. } \\
\text { II, H, i) }\end{array}$ & - & $\begin{array}{l}\text { Posterior vertical } \\
\text { plate of prosternal } \\
\text { apophysis }\end{array}$ & - & $\begin{array}{l}\text { Profurcal arm } \\
\text { (part.) }\end{array}$ & $\begin{array}{l}\text { Profurcal arm } \\
\text { (part.) }\end{array}$ & $\begin{array}{l}\text { Posterior vertical } \\
\text { plate of the profurcal } \\
\text { arm** }\end{array}$ \\
\hline Epimerum & Epimeral strip & Epimeron & - & Proepimeral list & - & - & Proepimeral list \\
\hline Ridge j (Fig. 17) & - & - & - & - & $\begin{array}{l}\text { Longitudinal } \\
\text { propleural carina }\end{array}$ & Propleural carina & Propleural carina \\
\hline- & - & - & Pleural ridge I (?) & Propleural ridge & Propleural ridge* & - & $\begin{array}{l}\text { Propleural ridge } \\
(?)^{* *}\end{array}$ \\
\hline Pleural suture & Pleural sulcus & Pleural sulcus & - & Propleural sulcus & Propleural sulcus* & - & Propleural sulcus (?) \\
\hline $\begin{array}{l}\text { Propleurum } \\
\text { (episternum+epimerum) }\end{array}$ & - & $\begin{array}{l}\text { Prothoracic pleuron } \\
\text { (episternum+epimeron) }\end{array}$ & Propleuron & Propleuron & Propleuron & Propleuron & Propleuron \\
\hline 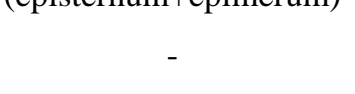 & - & Divergent arms* & $\begin{array}{l}\text { Prosternal } \\
\text { apophyseal arm }\end{array}$ & Profurcal arm & Profurcal arm & Profurcal arm & Profurcal arm** \\
\hline- & $\begin{array}{l}\text { Bridge* } \\
\text { (Fig. II, I, J, m) }\end{array}$ & Horizontal bridge* & $\begin{array}{l}\text { Prosternal } \\
\text { apophyseal bridge }\end{array}$ & Profurcal bridge & Profurcal bridge & Profurcal bridge & Profurcal bridge** \\
\hline- & (2) & - & $\begin{array}{l}\text { Prosternal } \\
\text { apophyseal pit }\end{array}$ & Profurcal pit & Profurcal pit & Profurcal pit & Profurcal pit** \\
\hline Prosternal furca & $\begin{array}{l}\text { Prothoracic } \\
\text { endosternum }\end{array}$ & $\begin{array}{l}\text { Endosternum } \\
\text { (of prothorax) }\end{array}$ & Prosternal apophysis & Profurca & Profurca & Profurca & Profurca** \\
\hline- & 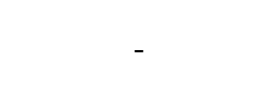 & 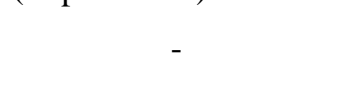 & - & $\begin{array}{l}\text { Prothoracic } \\
\text { katepisternum }\end{array}$ & $\begin{array}{l}\text { Prothoracic } \\
\text { katepisternum }\end{array}$ & - & $\begin{array}{l}\text { Prothoracic } \\
\text { katepisternum }\end{array}$ \\
\hline- & - & - & - & - & $\begin{array}{l}\text { Pseudophragmal } \\
\text { lobe }\end{array}$ & - & Pseudophragmal lobe \\
\hline- & - & - & - & - & 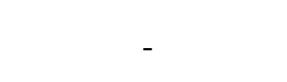 & - & $\begin{array}{l}\text { Ridge of the } \\
\text { propleural arm** }\end{array}$ \\
\hline
\end{tabular}


Table 2. Continuation

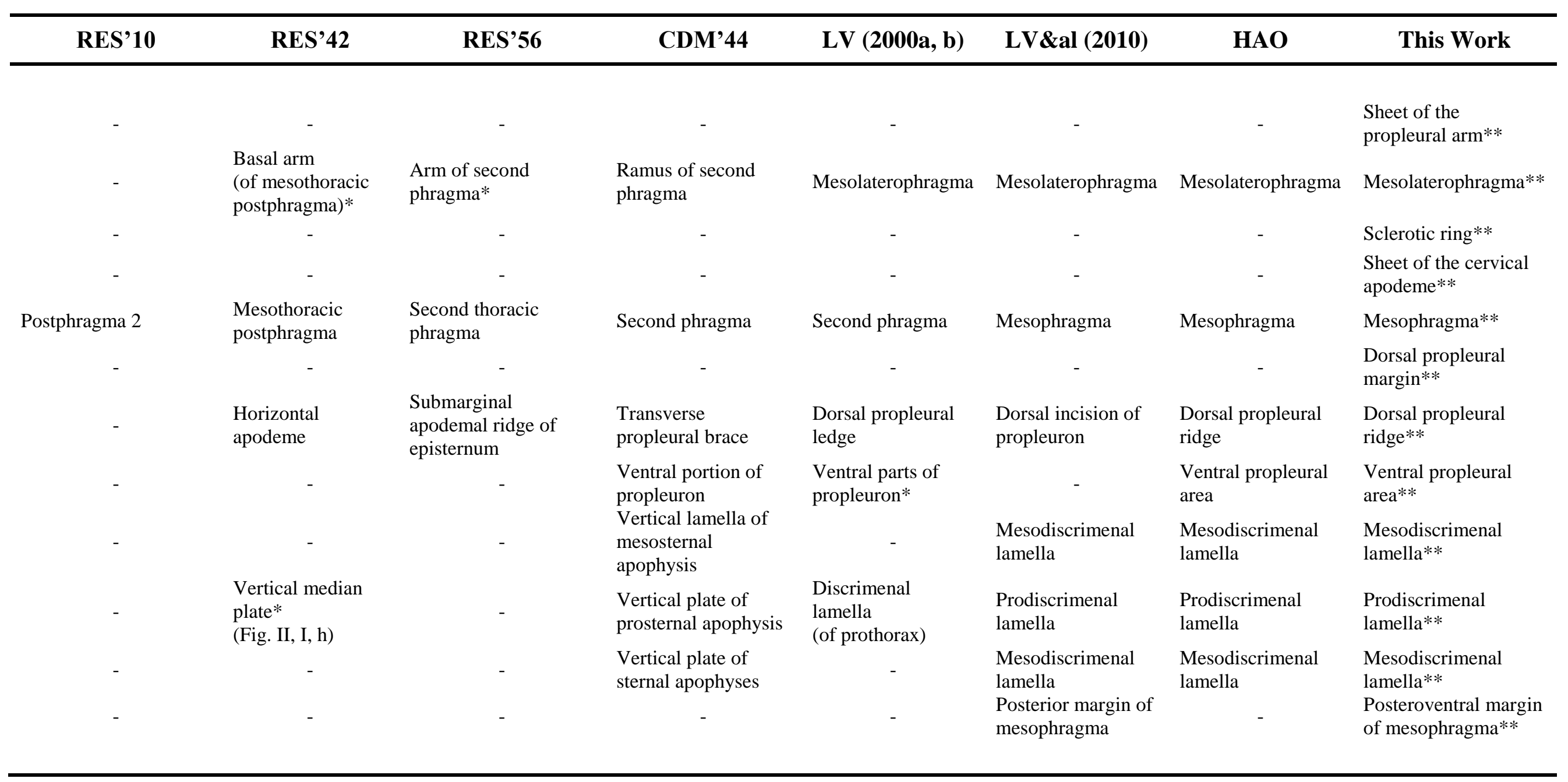


Table 3. Matrix of character states distribution. - = inapplicable. Abbreviations: ANDR, Andrenidae; APID, Apidae; COLL, Colletidae; HALI, Halictidae; MEGA, Megachilidae; MELI, Melittidae; STEN, Stenotritidae.

\begin{tabular}{|c|c|c|c|c|c|c|c|c|c|c|c|c|c|c|c|c|c|c|c|c|c|c|c|c|c|c|c|c|c|}
\hline & \multicolumn{28}{|c|}{ Characters } \\
\hline & & 0 & & & & & & & & & & & & & & & & & & & & & & & & & & & \\
\hline & & 1 & & 3 & & 5 & 6 & 7 & 8 & 9 & 0 & 1 & & & 4 & & 6 & & 8 & 9 & 0 & 1 & 2 & 3 & 4 & & & & \\
\hline MELI & syp & 0 & 0 & 0 & 0 & 0 & 0 & 0 & 0 & 1 & & 0 & 1 & 0 & 1 & & & & 0 & 0 & 0 & 1 & 0 & & 0 & & & & \\
\hline MELI & ghami & & 0 & 0 & 0 & 0 & 0 & 0 & 0 & 1 & - & 0 & & 0 & 1 & & & & 0 & 0 & 0 & 1 & 0 & & 0 & & & & \\
\hline MELI & elitta haen & 0 & 0 & 0 & 0 & 0 & 0 & 0 & 0 & 1 & - & 0 & 1 & 0 & 0 & 0 & 0 & & 1 & 0 & 0 & 1 & 0 & 0 & 0 & & 0 & & \\
\hline ANDR & & 0 & 0 & 0 & 0 & 0 & 0 & 0 & 0 & 0 & 0 & 0 & 0 & 0 & U & 0 & & J & 0 & 0 & 0 & 1 & 0 & & & U & & & \\
\hline ANDR & $I$ & 0 & 0 & 0 & 0 & 0 & 0 & 0 & 0 & 0 & 0 & 0 & 0 & 0 & 1 & 0 & 0 & & 1 & 0 & 0 & 1 & 0 & & 0 & 0 & & & \\
\hline ANDR & Pro & ) & 0 & 0 & 0 & 1 & 0 & 0 & 0 & 0 & 0 & 0 & 0 & 0 & 2 & 1 & 0 & J & 0 & 0 & 0 & 1 & 0 & & & & 0 & & U \\
\hline HALI & Penc & 0 & 0 & 0 & 0 & 0 & 0 & 0 & 0 & 0 & 0 & 0 & 0 & 0 & 1 & 0 & 0 & j & 0 & 0 & 0 & 1 & 0 & & 0 & 0 & & & \\
\hline HALI & $D$ & 0 & 0 & 0 & 0 & 0 & 0 & 0 & 0 & 0 & 0 & 0 & 0 & 0 & 2 & 0 & 0 & & 0 & 0 & 0 & 1 & 0 & & 0 & & & & \\
\hline HALI & Aga & U & 0 & 0 & 0 & 1 & 0 & 0 & 0 & 0 & 0 & 0 & 0 & 0 & 2 & 0 & 0 & J & 0 & 0 & 0 & 1 & 1 & & & & 0 & & 0 \\
\hline STEN & & & 0 & 0 & 0 & 0 & 0 & 0 & 0 & 0 & 0 & 0 & 0 & 0 & 1 & 1 & 1 & j & 1 & 0 & 0 & 1 & 1 & 0 & 0 & 0 & & & \\
\hline COLL & & & 0 & 0 & 0 & 1 & 0 & 0 & 0 & 0 & 0 & 0 & 0 & 0 & 0 & 0 & 0 & J & 1 & 0 & 0 & 1 & 0 & & 0 & & 0 & & 0 \\
\hline COLL & My & U & 0 & 0 & 0 & 1 & 0 & 0 & 0 & 0 & 0 & 0 & 0 & 0 & 0 & 0 & 1 & o & 0 & 0 & 0 & 1 & 0 & & 0 & & 0 & & \\
\hline $\mathrm{CO}$ & & & 0 & 0 & 0 & 1 & 0 & 0 & 0 & 0 & 0 & 0 & 0 & 0 & 0 & 0 & 0 & & 0 & 0 & 0 & 1 & 0 & & U & & & & \\
\hline COLL & $\mathrm{Col}$ & & 0 & 0 & 0 & 1 & 0 & 0 & 0 & 0 & 0 & 0 & 0 & 0 & 0 & 0 & 0 & $\mathrm{~J}$ & 1 & 1 & 0 & 1 & 0 & 1 & 0 & & 0 & 0 & 0 \\
\hline COLL & & 0 & 0 & 0 & 0 & 1 & 0 & 0 & 0 & 0 & 0 & 0 & 0 & 0 & 0 & 0 & 1 & & 0 & 1 & 0 & 1 & 0 & & & & & & \\
\hline & Lon & & 0 & 0 & 0 & 1 & 0 & 0 & 0 & 0 & 0 & 0 & 0 & 0 & 0 & 0 & 0 & U & 1 & 1 & 0 & 1 & 0 & 1 & 0 & U & & 0 & \\
\hline MEG & & & 0 & 0 & 0 & 0 & 0 & 0 & 0 & 1 & 0 & 0 & 0 & 1 & 0 & 0 & 1 & 0 & 0 & 0 & 0 & 1 & 0 & 0 & 0 & & 0 & 0 & \\
\hline MEGA & Lith & & 0 & 0 & 0 & 0 & 0 & 0 & 0 & 0 & 0 & 0 & 0 & 0 & 1 & 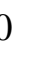 & 1 & & 0 & 0 & 0 & 1 & 1 & 0 & 0 & 0 & & 0 & \\
\hline MEGA & Ant & & 0 & 0 & 0 & 0 & 0 & 0 & 0 & 0 & 0 & 0 & 0 & 0 & 1 & 0 & 1 & 0 & 0 & 0 & 0 & 1 & 1 & 0 & 0 & & $\begin{array}{ll}0 & 1\end{array}$ & 1 & 1 \\
\hline APID & Aca & & 0 & 0 & 0 & - & 0 & 0 & 0 & - & 0 & 0 & 0 & 1 & 1 & 1 & 1 & & 0 & 0 & 0 & 0 & 0 & 0 & & & & 0 & \\
\hline APID & Ant & & 0 & 0 & 0 & 0 & 0 & 0 & 0 & 0 & - & 0 & 0 & 0 & 1 & 0 & 1 & U & 1 & 0 & 0 & 1 & 0 & 0 & 0 & 0 & & 1 & \\
\hline & $\mathrm{Mel}$ & & 0 & 0 & 0 & 0 & 0 & 0 & 0 & 1 & 0 & 0 & 0 & 0 & & & 1 & & 1 & 0 & 0 & 1 & 1 & 0 & 0 & 0 & 0 & 0 & 0 \\
\hline & & & 0 & 0 & 0 & 0 & 0 & 0 & 0 & 1 & 0 & 0 & 0 & 0 & & J & 1 & & 0 & 0 & 0 & 1 & 1 & & & & & 0 & \\
\hline APID & Tapin & 0 & 0 & 0 & 0 & 0 & 0 & 0 & 0 & 1 & 0 & 0 & 0 & 0 & 1 & 0 & 1 & 0 & 0 & 0 & 0 & 1 & 1 & 0 & 1 & 1 & 0 & 0 & \\
\hline & & & $\Omega$ & 0 & & 0 & 0 & & & 0 & 0 & 0 & 0 & 1 & 0 & 0 & 1 & & 0 & 0 & 0 & 1 & 1 & & 0 & & 0 & 0 & \\
\hline APID & Man & & 0 & 0 & 0 & 0 & 0 & 0 & 0 & 0 & - & 0 & 0 & 0 & 1 & ) & 1 & & 0 & 0 & 0 & 1 & 0 & 0 & 1 & 0 & 00 & 0 & \\
\hline & Epic & & 0 & 0 & 0 & 0 & 0 & 0 & 0 & 0 & - & 0 & 0 & 0 & 2 & 1 & 1 & 0 & 1 & 0 & 0 & 1 & 1 & 0 & 1 & & 11 & 1 & \\
\hline & & & 0 & 0 & 0 & 0 & 0 & 0 & 0 & 0 & - & 0 & 0 & 0 & l & 1 & 1 & & 1 & 0 & 0 & 1 & 1 & 0 & & & 1 & 1 & \\
\hline APID & Centris analis & 0 & 0 & 0 & 0 & 0 & 0 & 0 & 0 & 0 & - & 0 & 0 & 0 & & 1 & 1 & ) & 1 & 0 & 0 & 1 & 1 & 0 & 1 & 0 & 11 & 1 & \\
\hline API & Exae & & & 0 & 1 & 0 & 0 & 1 & 0 & 1 & 0 & 0 & 0 & 0 & & & 1 & & 0 & 0 & 1 & 1 & 1 & 0 & 0 & & 1 & 1 & \\
\hline API & Eufriesea violaced & & 0 & 0 & 1 & 0 & 0 & 1 & 0 & 1 & 1 & 1 & 0 & 0 & 0 & 1 & 1 & & 1 & 0 & 1 & 1 & 1 & 0 & & 0 & 1 & & \\
\hline API & Eulaema nigrita & & & 0 & 1 & 0 & 0 & & 0 & 1 & 1 & 1 & 0 & 0 & & & 1 & ) & 1 & 0 & 1 & 1 & 1 & 0 & 1 & 0 & & & \\
\hline & & & 0 & 0 & 1 & 0 & 0 & 1 & 0 & 1 & 1 & 1 & 0 & 1 & & & | & & 1 & 0 & 1 & - & 0 & 0 & & 0 & 10 & 0 & \\
\hline API & Bomb & & & 0 & 1 & 0 & 0 & 0 & & 1 & - & 0 & 0 & & & & & & 0 & 0 & 0 & 1 & 1 & 0 & 0 & 0 & 0 & 0 & \\
\hline API & Bombus ternarius & 1 & & 0 & 1 & 0 & 0 & 0 & 0 & 1 & - & 0 & 0 & 0 & 1 & 0 & 1 & 1 & 0 & 0 & 0 & 1 & 1 & 0 & & & & & \\
\hline
\end{tabular}


Table 3. Continuation.

\section{Characters}

$\begin{array}{llllllllllllllllllllllllllllll}\text { Taxa } & \mathbf{0} & \mathbf{0} & \mathbf{0} & \mathbf{0} & \mathbf{0} & \mathbf{0} & \mathbf{0} & \mathbf{0} & \mathbf{0} & 1 & 1 & 1 & 1 & 1 & 1 & 1 & 1 & 1 & 1 & 2 & 2 & 2 & 2 & 2 & 2 & 2 & 2 & 2\end{array}$

\begin{tabular}{llllllllllllllllllllllllllll}
1 & 2 & 3 & 4 & 5 & 6 & 7 & 8 & 9 & 0 & 1 & 2 & 3 & 4 & 5 & 6 & 7 & 8 & 9 & 0 & 1 & 2 & 3 & 4 & 5 & 6 & 7 & 8 \\
\hline
\end{tabular}

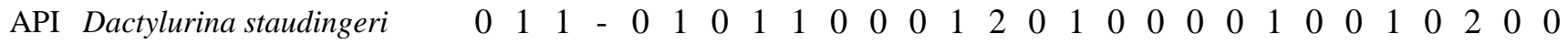

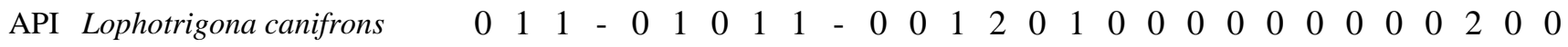

API Trigonisca nataliae

$\begin{array}{llllllllllllllllllllllllllll}0 & 1 & 1 & - & 0 & 1 & 0 & 1 & 1 & 0 & 0 & 0 & 1 & 2 & 0 & 1 & 0 & 0 & 0 & 0 & 0 & 0 & 0 & 1 & 0 & 2 & 0 & 0\end{array}$

API Melipona quadrifasciata

$\begin{array}{llllllllllllllllllllllllllll}0 & 1 & 1 & - & 0 & 1 & 0 & 1 & 1 & 0 & 0 & 0 & 1 & 2 & 0 & 1 & 0 & 0 & 0 & 0 & 1 & 0 & 0 & 1 & 0 & 2 & 0 & 0\end{array}$

API Tetragonisca angustula

$\begin{array}{llllllllllllllllllllllllllll}0 & 1 & 1 & - & 0 & 1 & 0 & 1 & 1 & - & 0 & 0 & 1 & 2 & 0 & 1 & 0 & 0 & 0 & 0 & 0 & 0 & 0 & 0 & 0 & 2 & 0 & 0\end{array}$

API Scaptotrigona depilis

$\begin{array}{llllllllllllllllllllllllllll}0 & 1 & 1 & - & 0 & 1 & 0 & 1 & 1 & - & 0 & 0 & 1 & 2 & 0 & 1 & 0 & 0 & 0 & 0 & 0 & 0 & 0 & 0 & 0 & 2 & 0 & 0\end{array}$

API Trigona hyalinata

$\begin{array}{llllllllllllllllllllllllllll}0 & 1 & 1 & - & 0 & 1 & 0 & 0 & 1 & - & 0 & 0 & 1 & 2 & 0 & 1 & 0 & 0 & 0 & 0 & 1 & 0 & 0 & 1 & 0 & 2 & 0 & 0\end{array}$

API Apis dorsata

$\begin{array}{llllllllllllllllllllllllllll}0 & 1 & 1 & - & 0 & 1 & 0 & 1 & 1 & 0 & 0 & 0 & 1 & 1 & 0 & 1 & 0 & 0 & 0 & 0 & 0 & 0 & 0 & 0 & 0 & 2 & 0 & 0\end{array}$

API Apis mellifera

$\begin{array}{llllllllllllllllllllllllllll}0 & 1 & 1 & - & 0 & 1 & 0 & 1 & 1 & 0 & 0 & 0 & 1 & 1 & 0 & 1 & 0 & 0 & 0 & 0 & 0 & 0 & 0 & 0 & 0 & 2 & 0 & 0\end{array}$ 

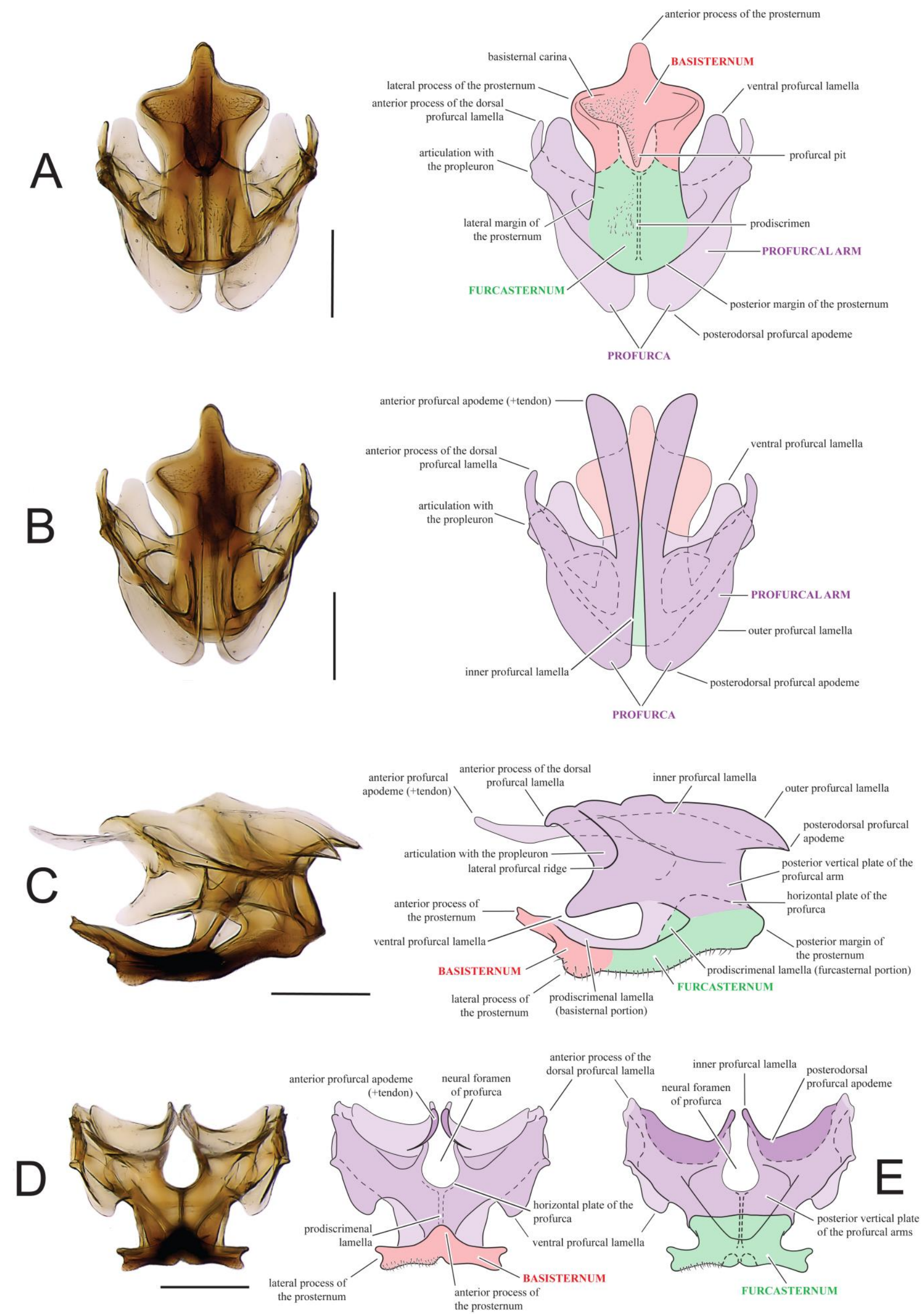

Figure 1. Prosternum of Centris analis (Fabricius, 1804). A. Ventral view, anterior to the top; B. Dorsal view, anterior to the top; C. Lateral view, anterior to the left; D. Anterior view; E. Posterior view. Scale bar $=0.5 \mathrm{~mm}$ 

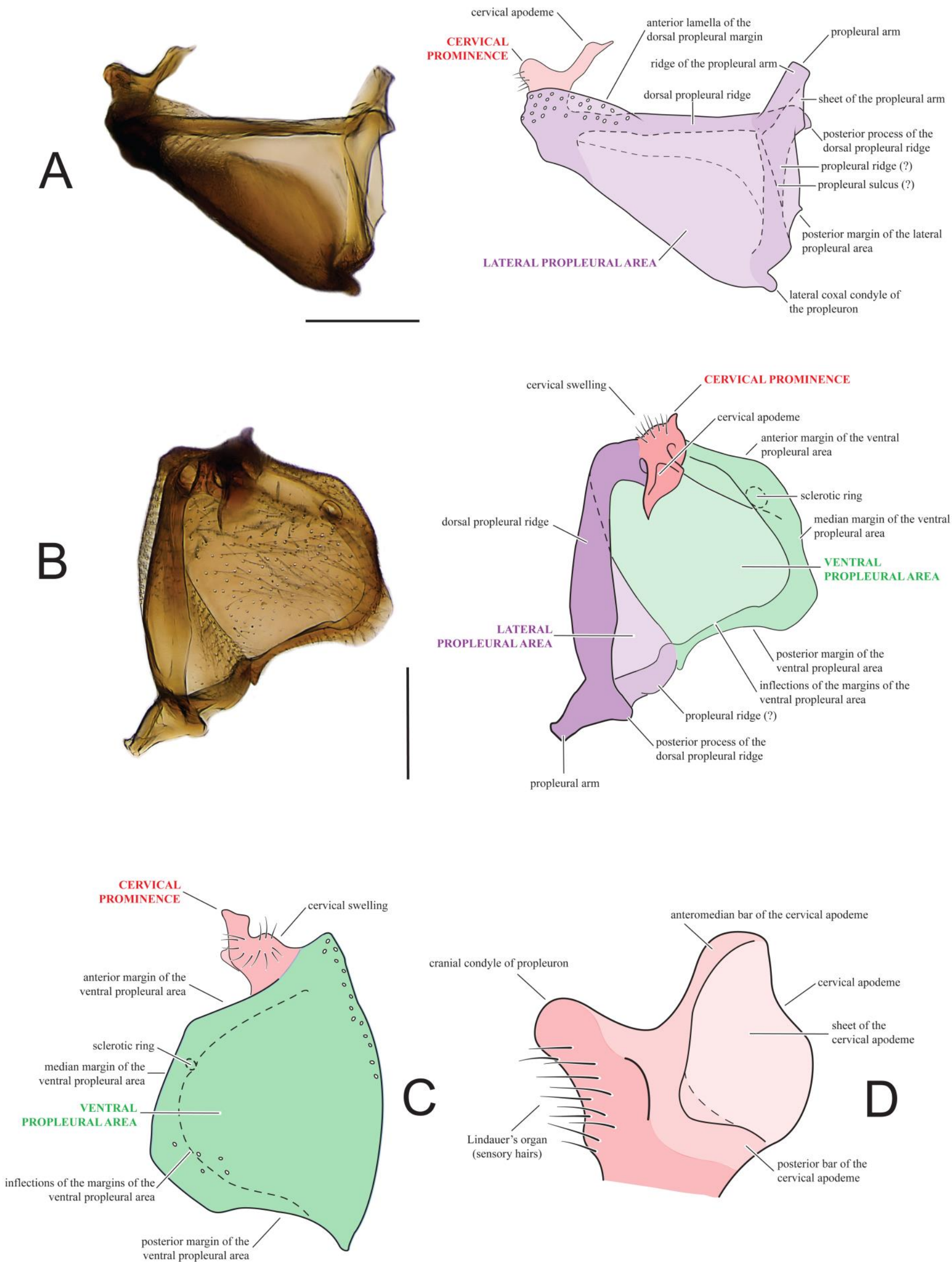

Figure 2. Propleuron of Centris analis (Fabricius, 1804). A. Lateral view, anterior to the left; B. Dorsal view, anterior to the top; $\mathbf{C}$. Ventral view, anterior to the top; D. Cervical prominence; latero-dorsal view, anterior to the left. Scale bar = $0.5 \mathrm{~mm}$ 

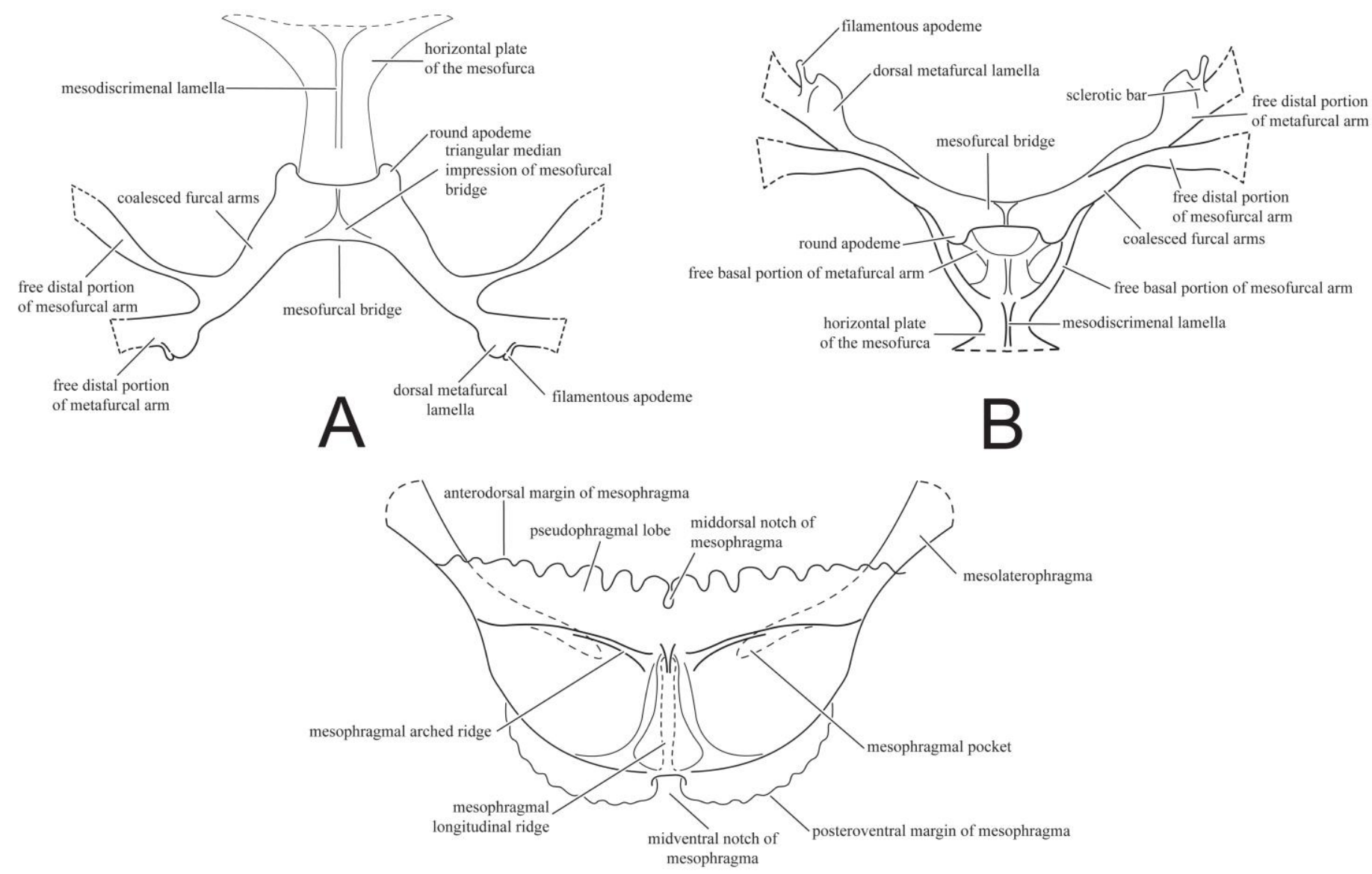

Figure 3. Mesofurcae/metafurcae and mesophragma of Centris analis (Fabricius, 1804). A-B Mesofurca/metafurca: A. Dorsal view, anterior to the top; B. Anterior view. C. Mesophragma, posterior view. 

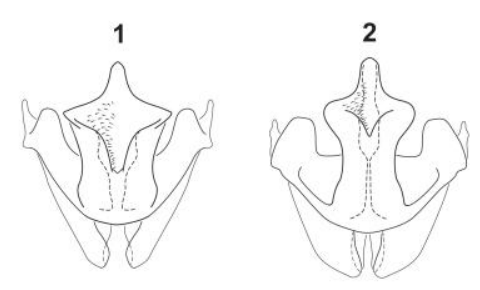

9
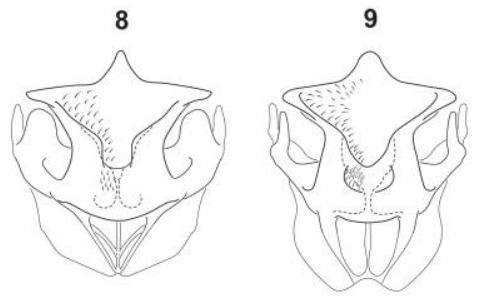

15
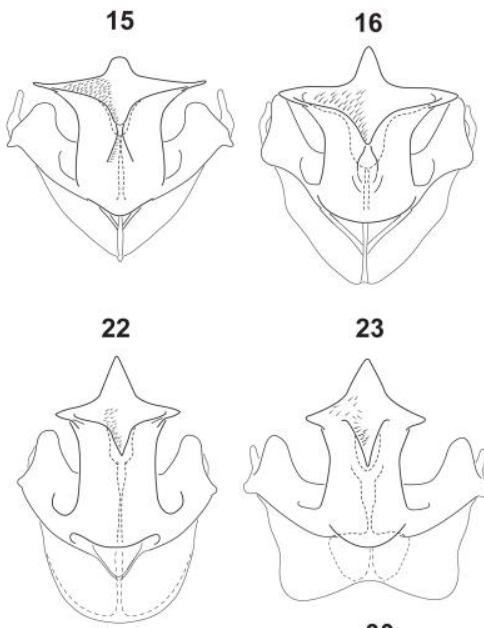

30
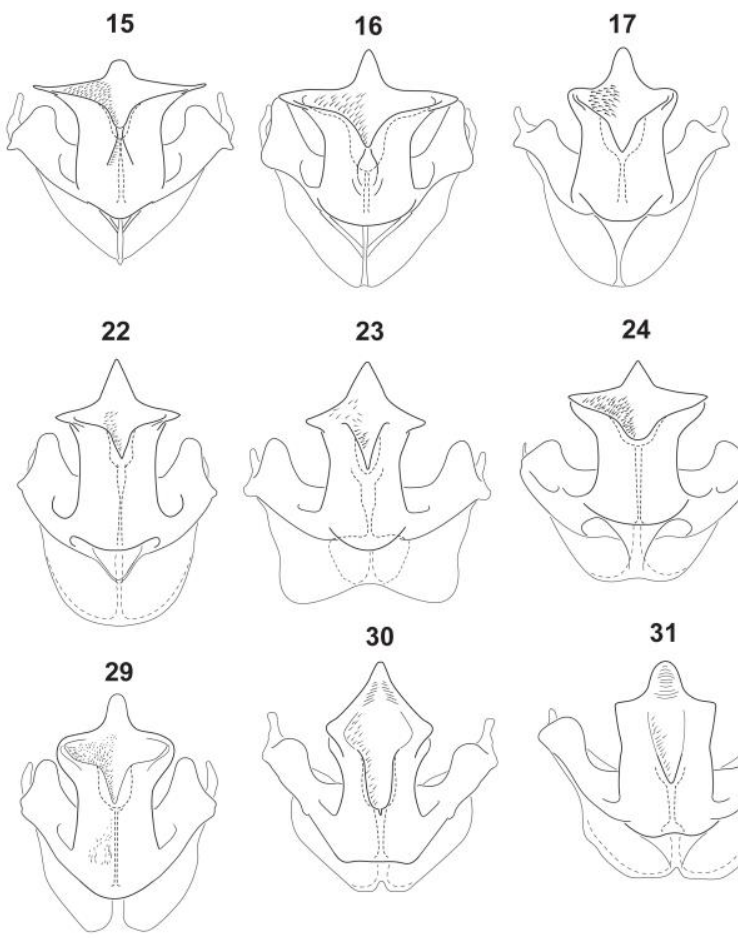

24
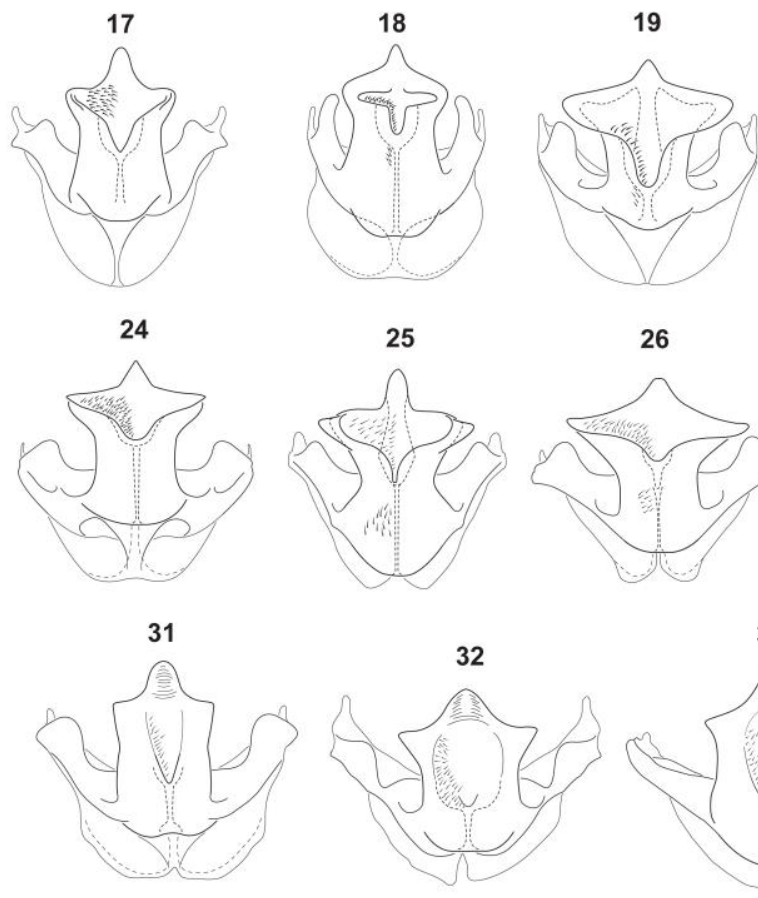

26
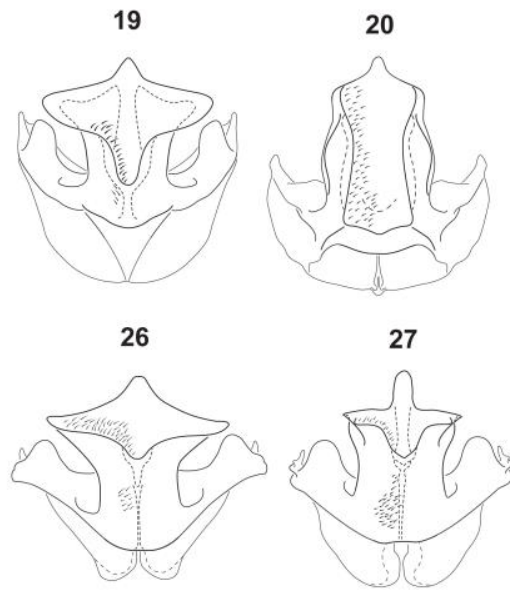

27
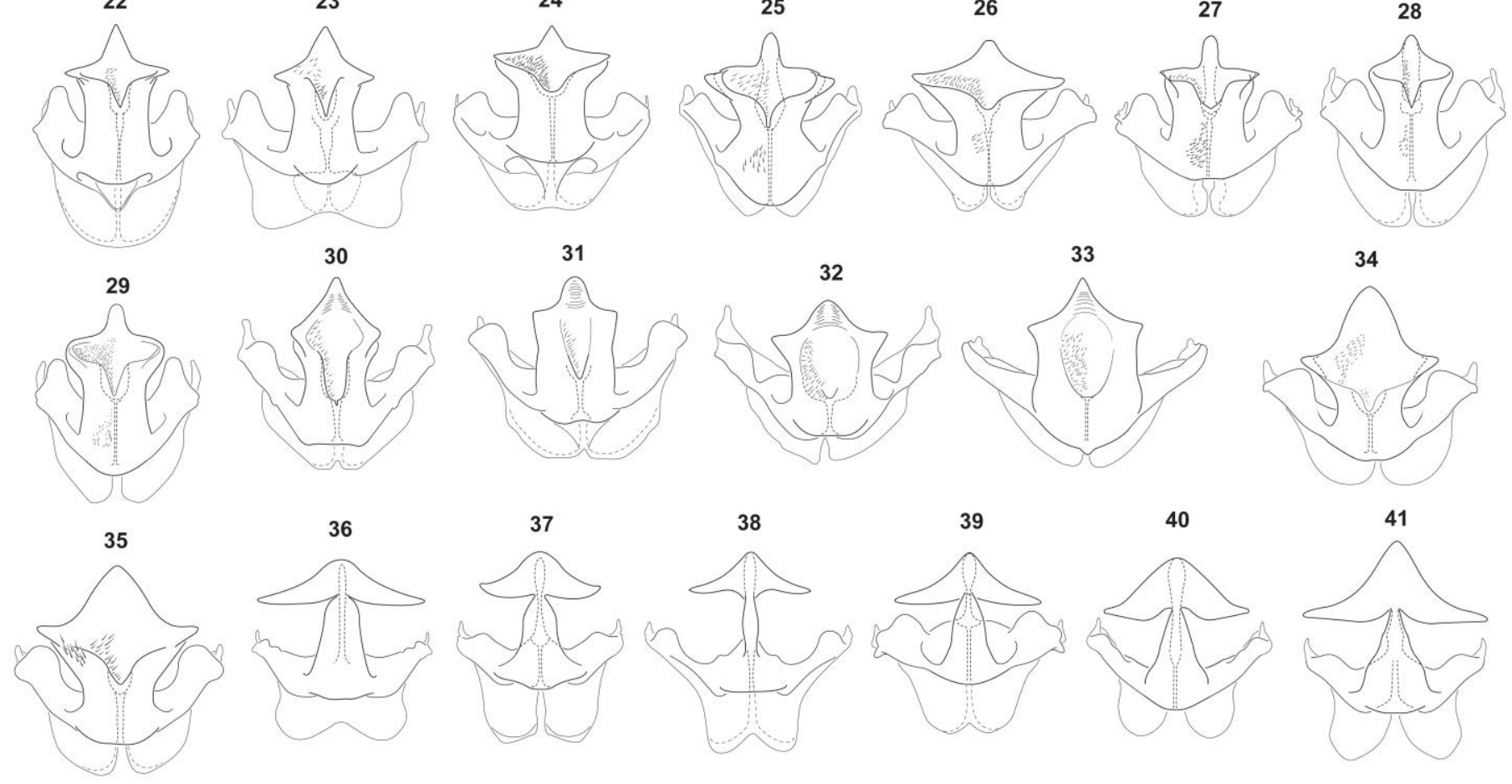
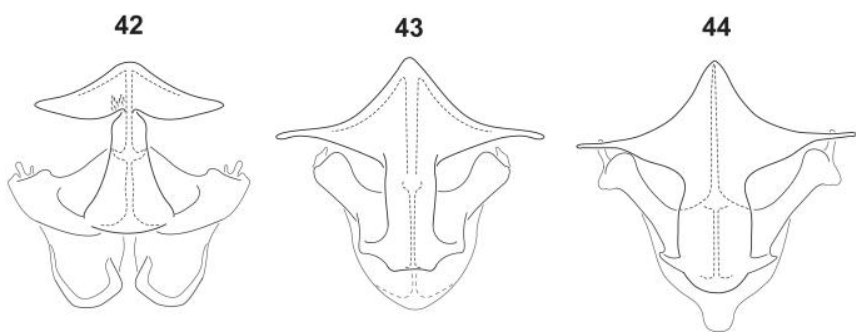

Figure 4. Prosternum; ventral view, anterior to the top; reference numbers for taxa as in Table 1. 


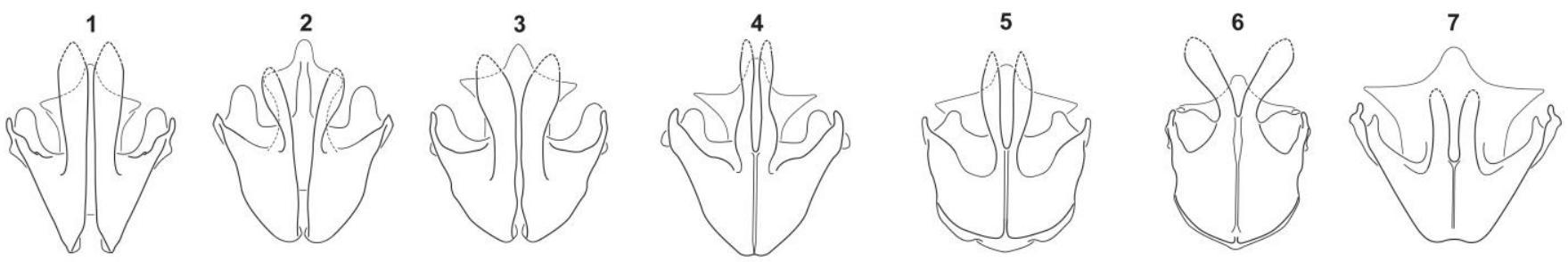
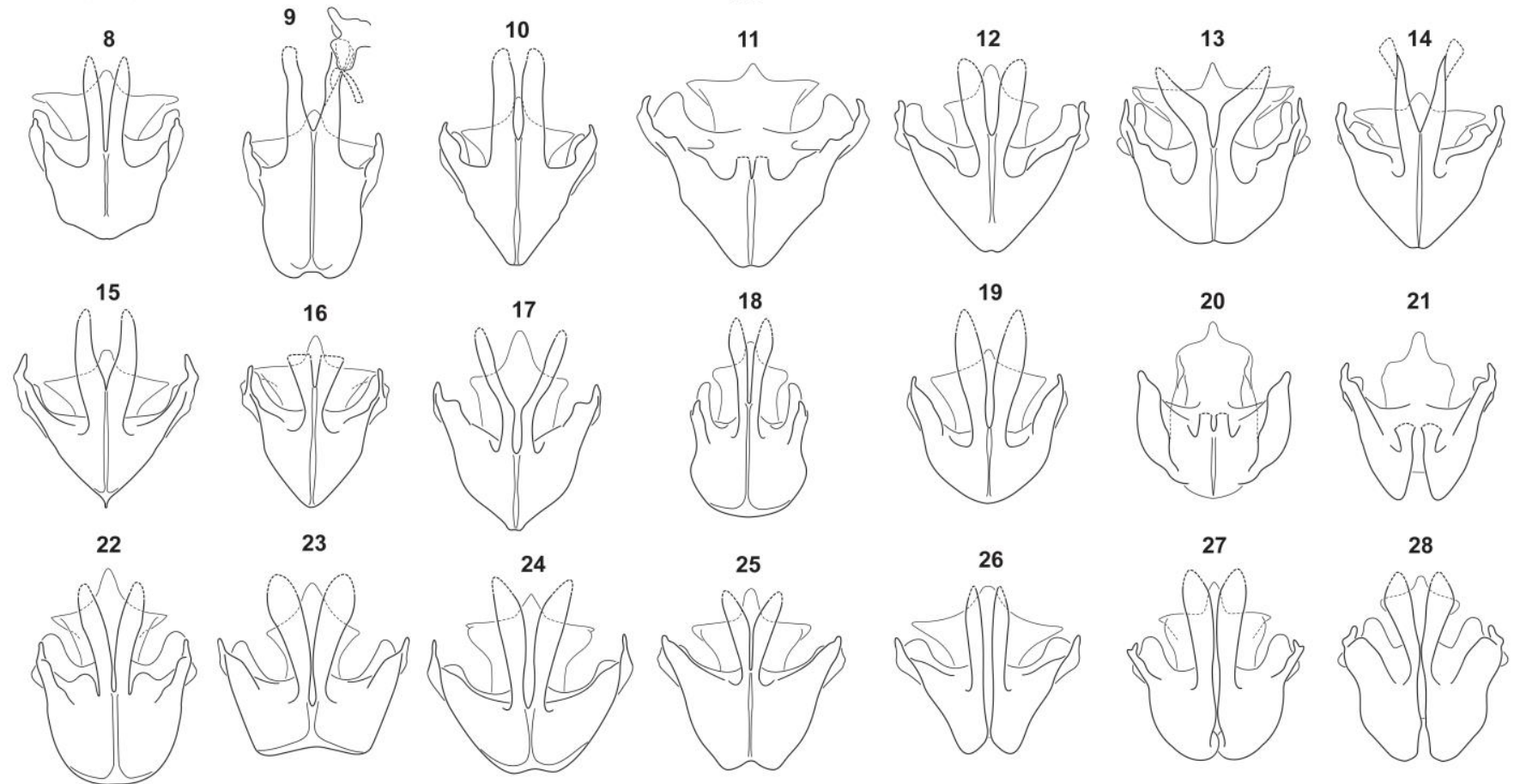

s

"

${ }^{25}$
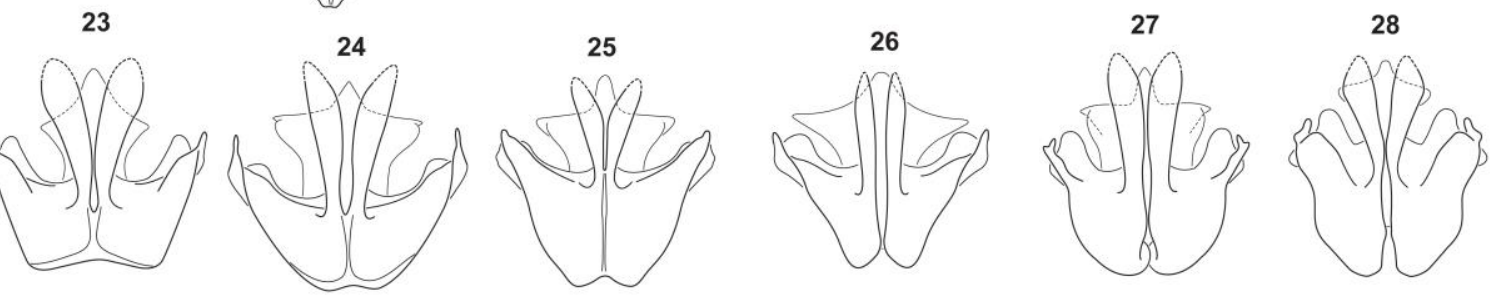

32

33

34

35
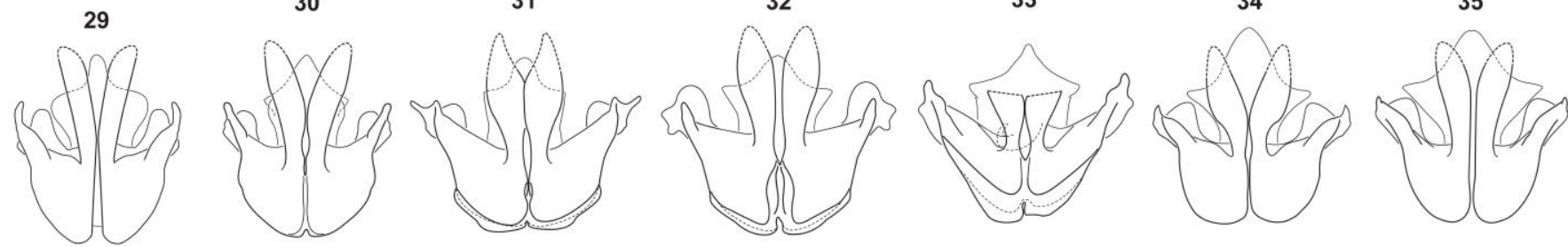

36

37
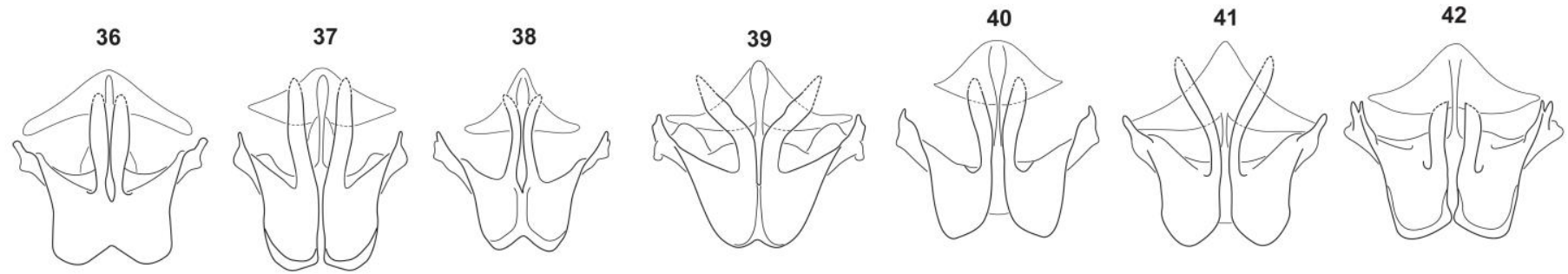

43

44

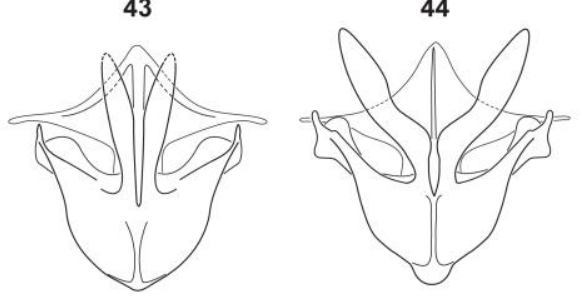

Figure 5. Prosternum; dorsal view, anterior to the top; reference numbers for taxa as in Table 1. 

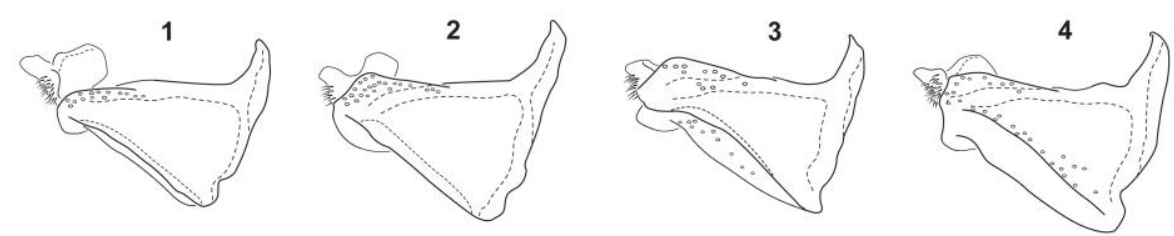

9
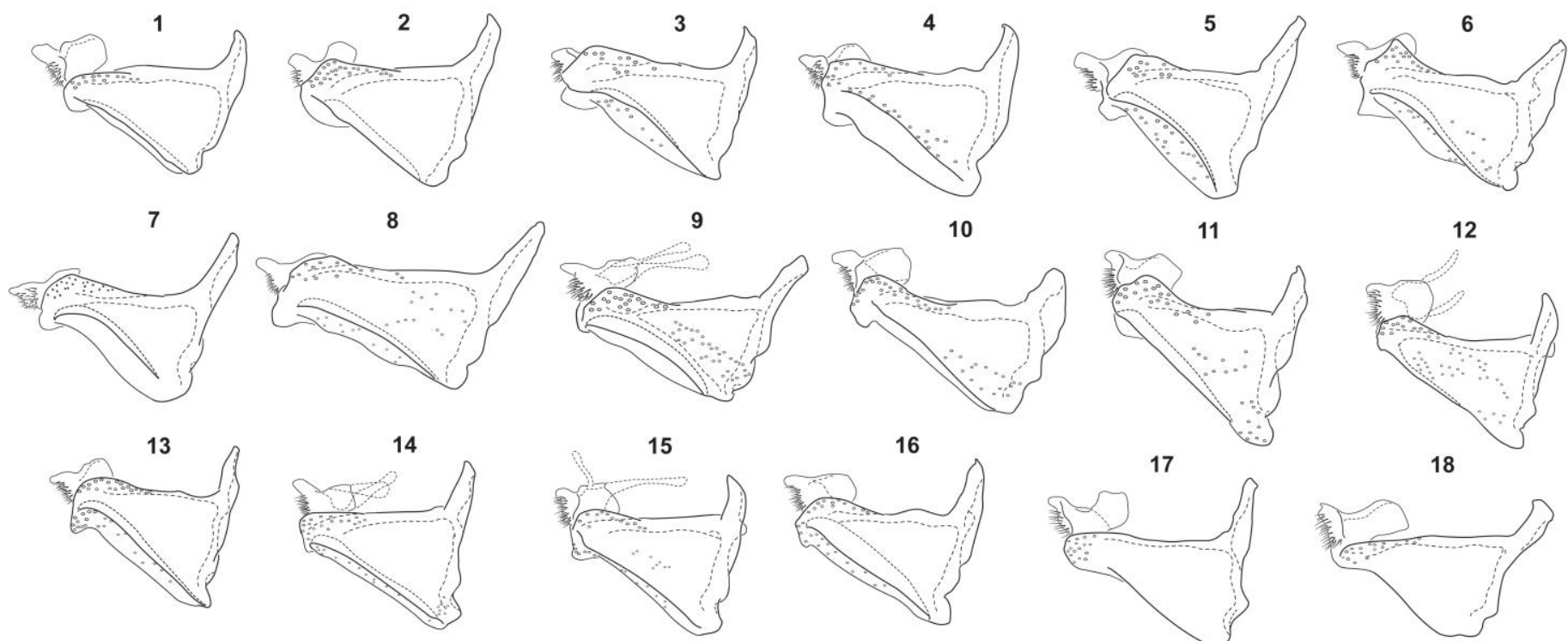

21
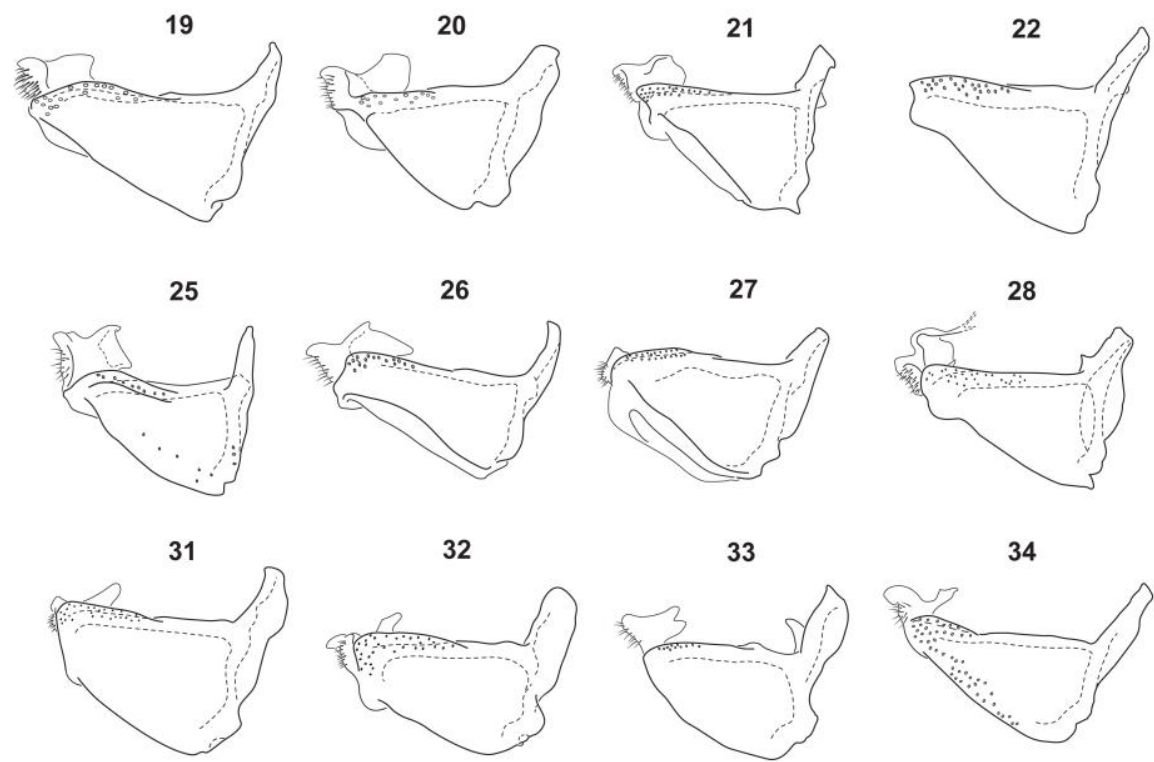

32

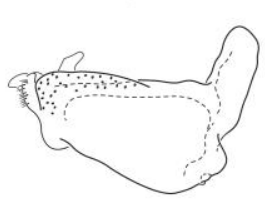

38
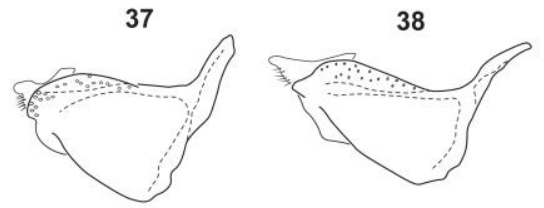

44
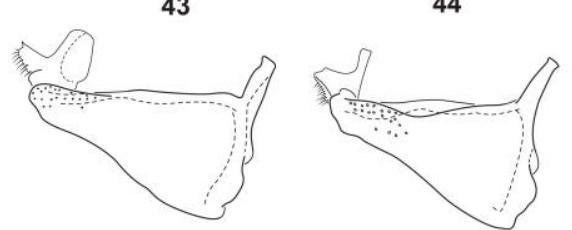

27

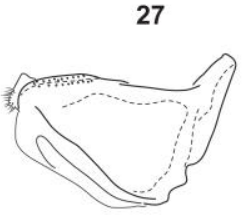

33

28

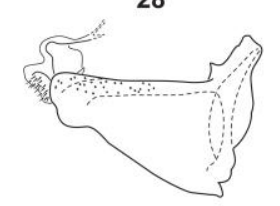

34

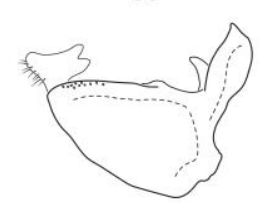

39
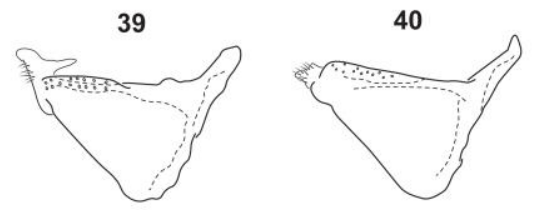

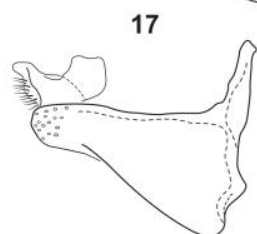

23

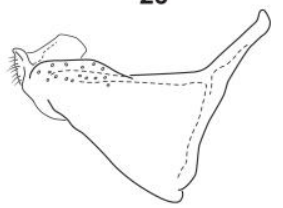

29

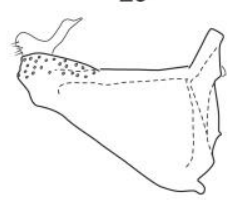

35

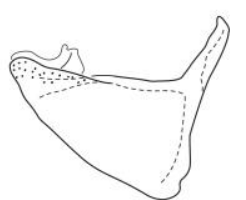

41

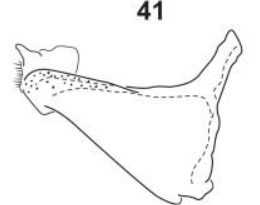

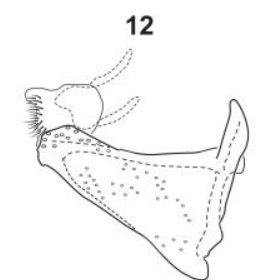

18

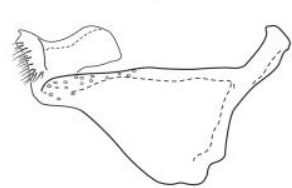

24

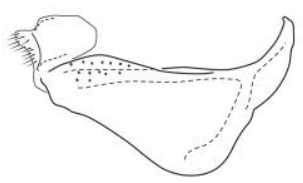

30

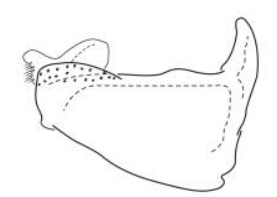

36

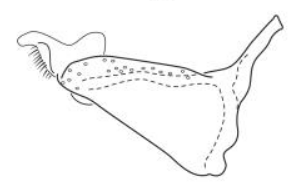

42

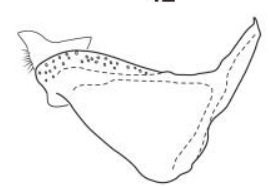

Figure 6. Propleuron; lateral view, anterior to the left; reference numbers for taxa as in Table 1. 

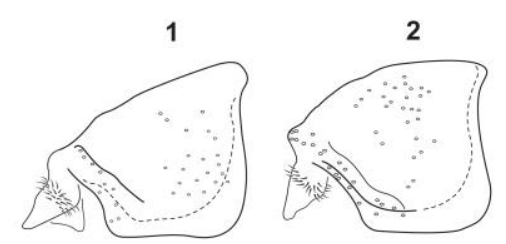

9
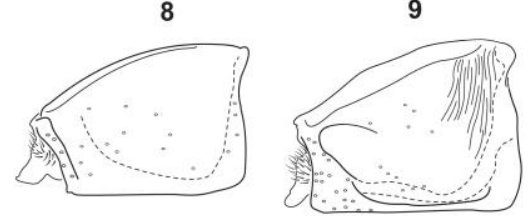

16
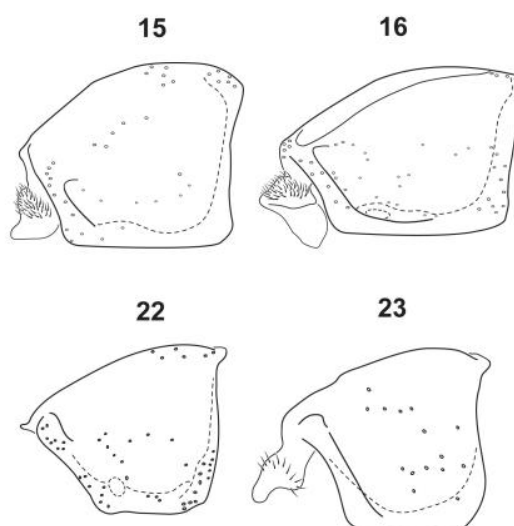

29
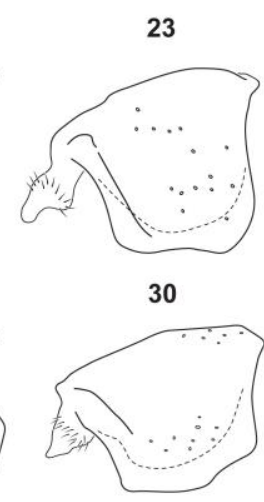

37
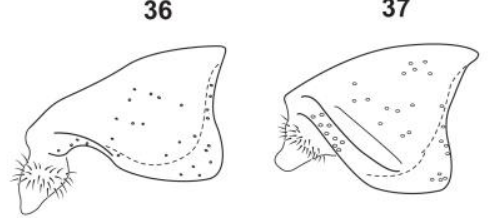

43
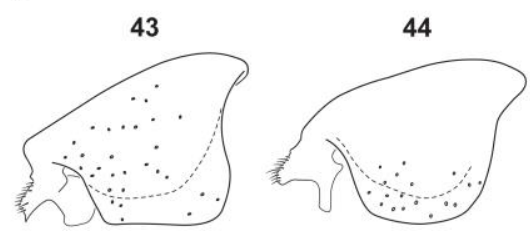
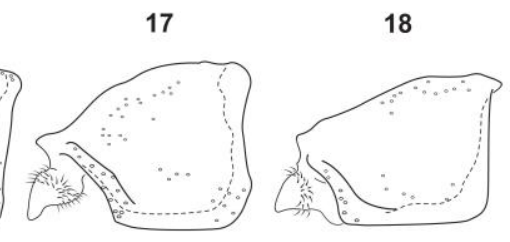

24
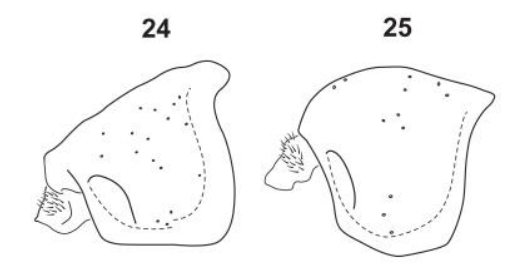

31

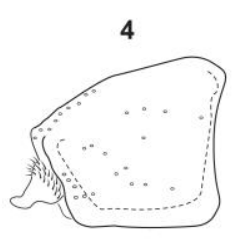

11
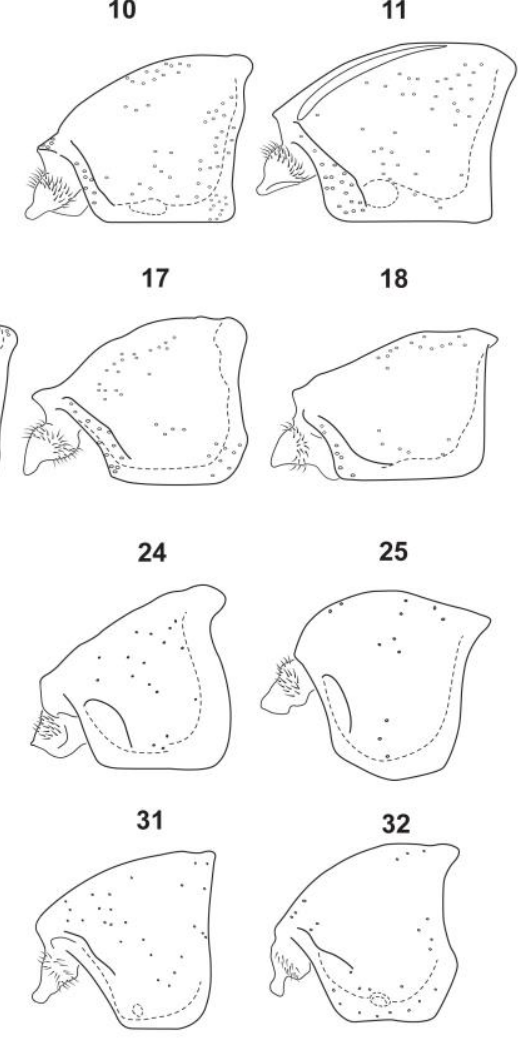

38
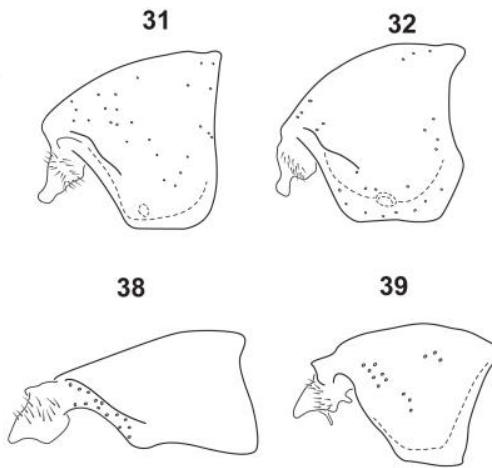

39

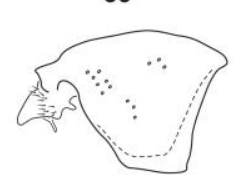

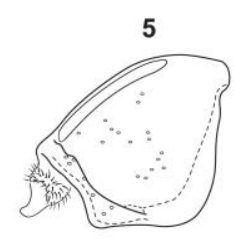

12

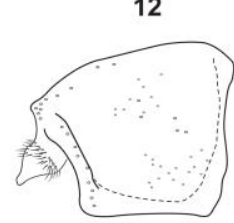

19

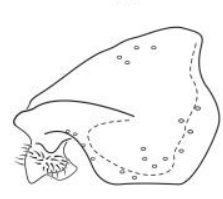

26

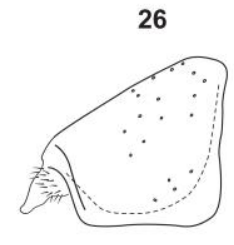

33

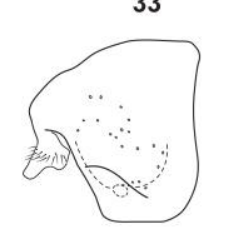

40

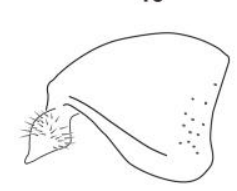

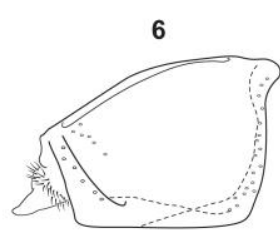

13

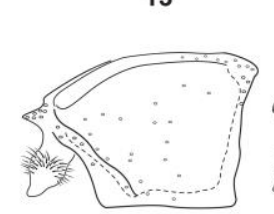

20

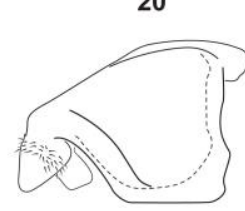

27
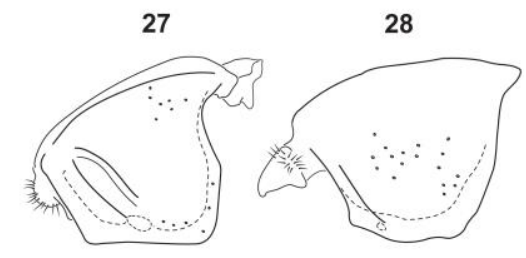

34

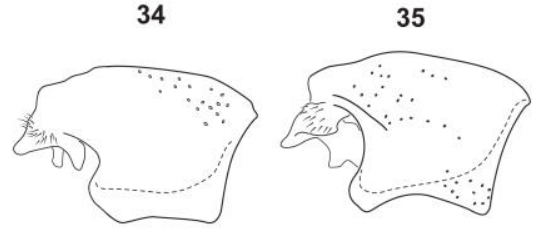

41

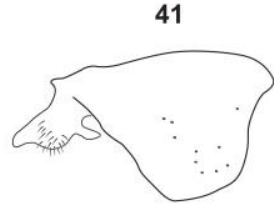

42

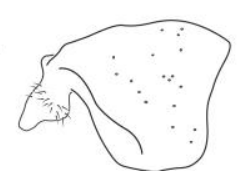

Figure 7. Propleuron; ventral view, anterior to the left; reference numbers for taxa as in Table 1. 


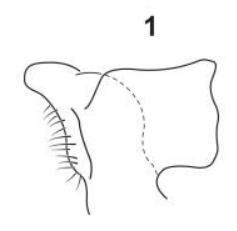

2

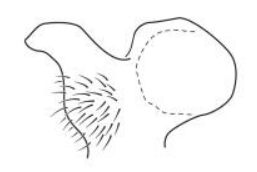

3

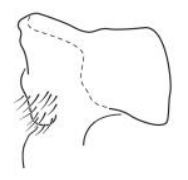

4

5

6

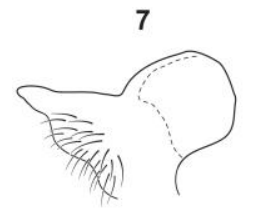

8

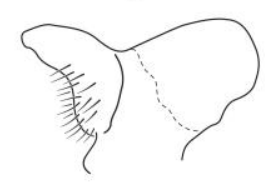

9

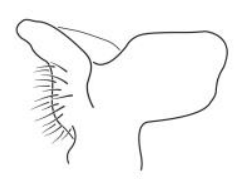

15
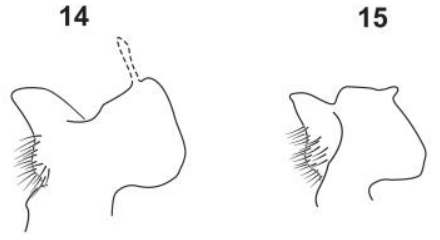

19

20
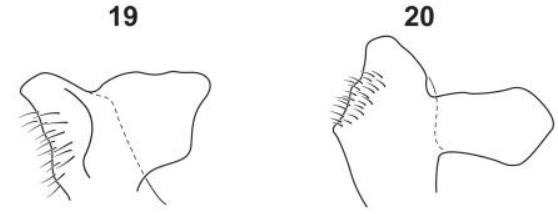

21

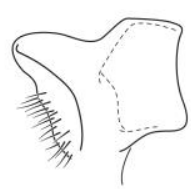

27
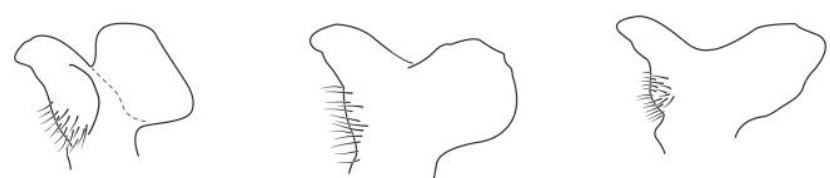

10
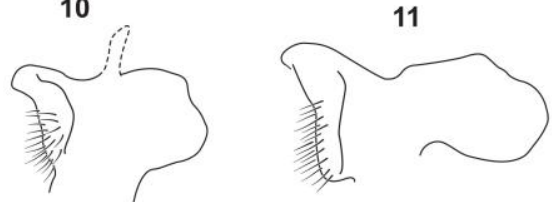

12

26

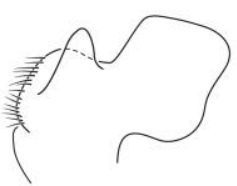

33
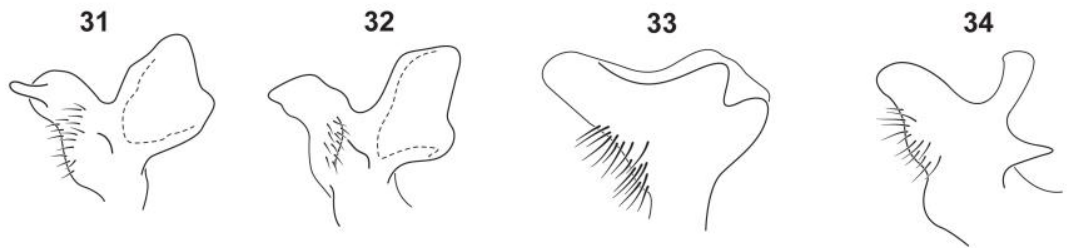

37
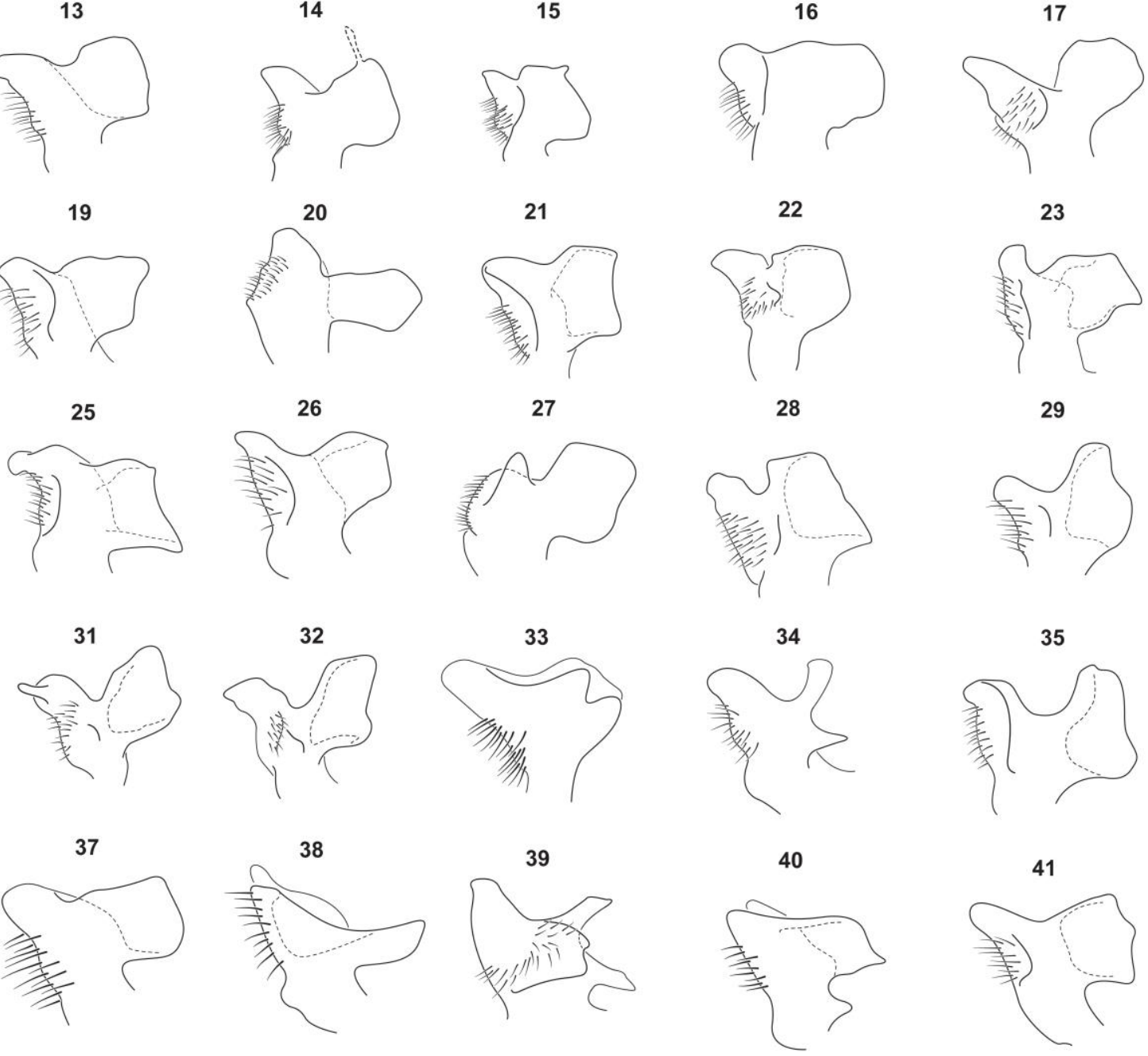

23

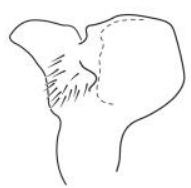

28

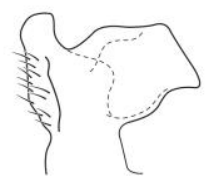

29

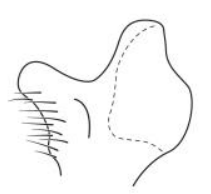

35

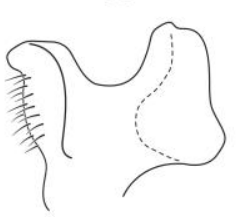

41

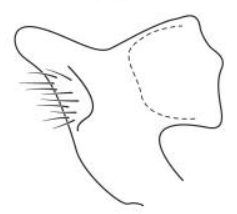

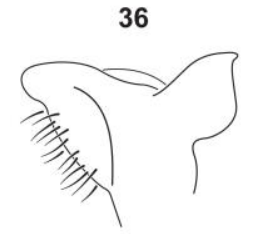

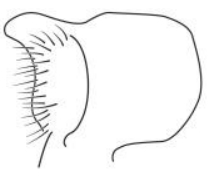

18

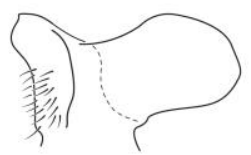

24

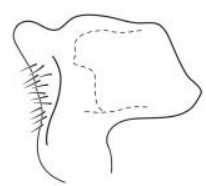

30

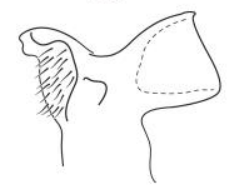

42

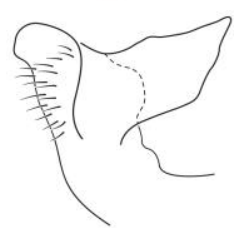

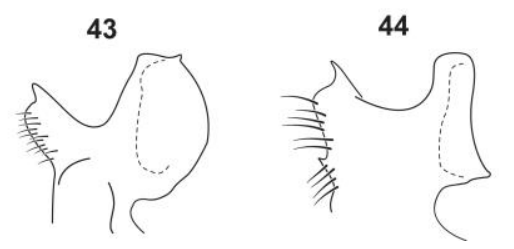

Figure 8. Cervical prominence; latero-dorsal view, anterior to the left; reference numbers for taxa as in Table 1. 


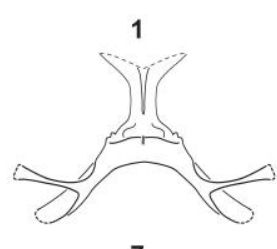

7
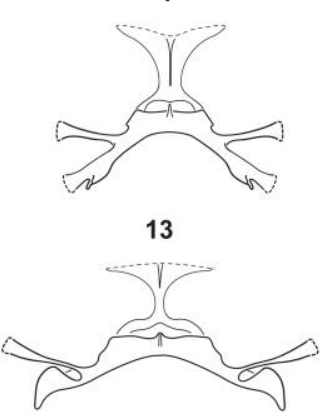

19

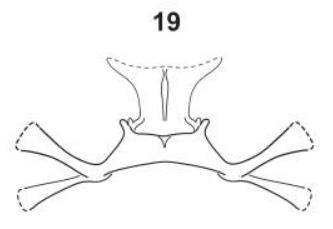

25

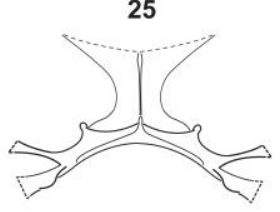

31

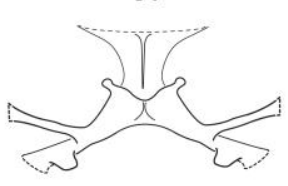

37

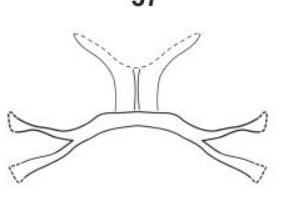

43

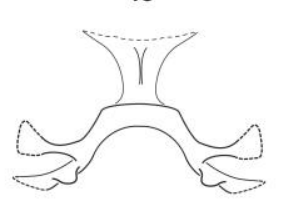

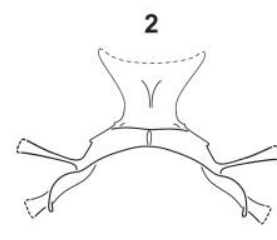

8

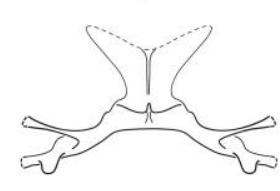

14

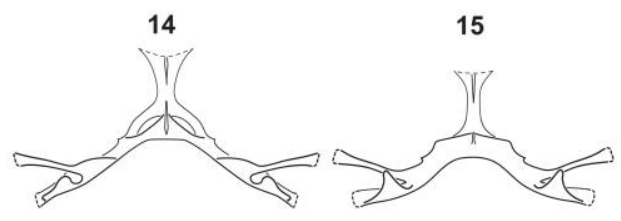

20

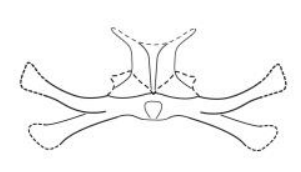

26

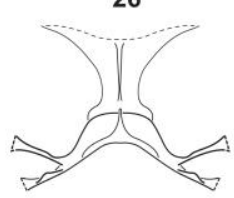

32

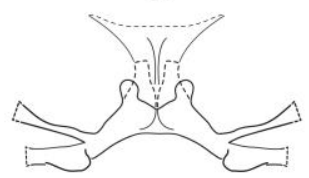

38

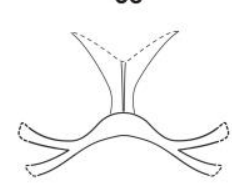

44

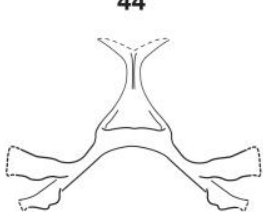

21

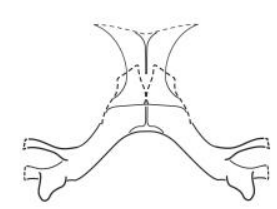

27

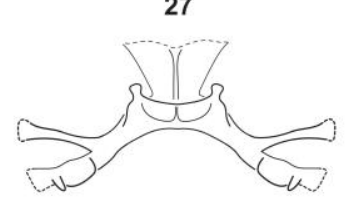

33

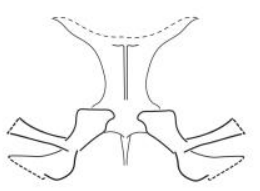

39

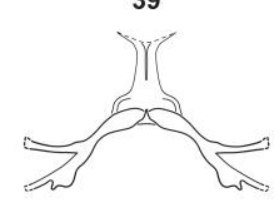

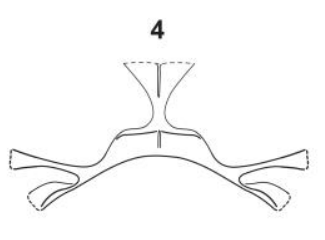

10

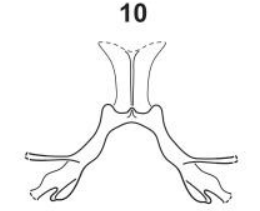

16

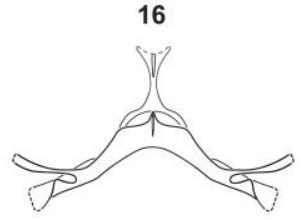

22

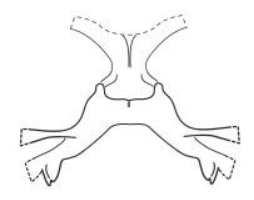

28

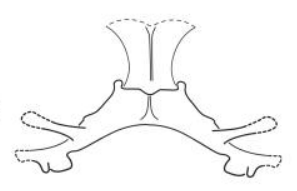

34

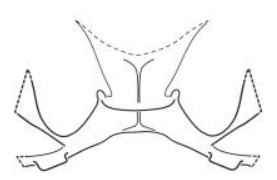

40

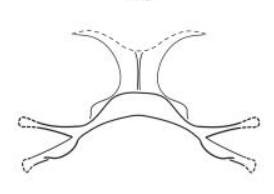

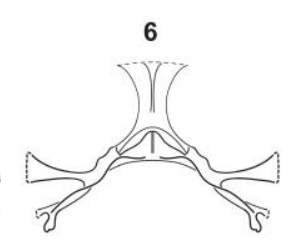

12

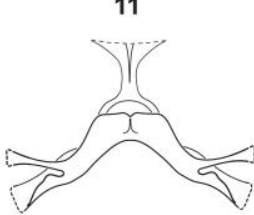

17

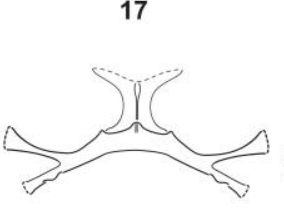

23

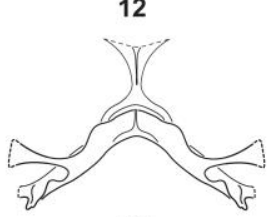

18

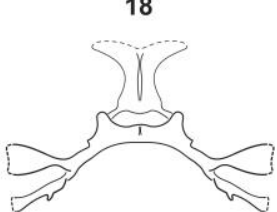

24
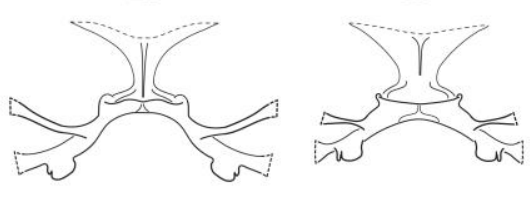

29

30

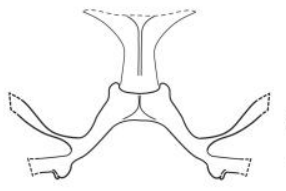

35

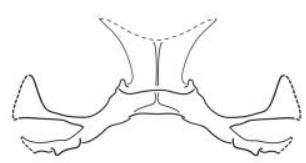

41
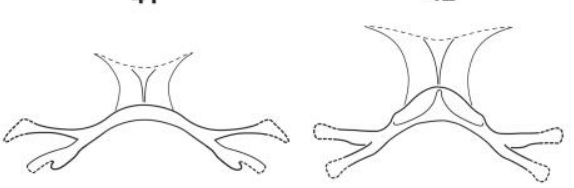

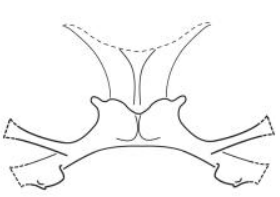

36

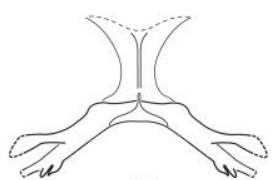

42

Figure 9. Mesofurca/metafurca; dorsal view, anterior to the top; reference numbers for taxa as in Table 1. 

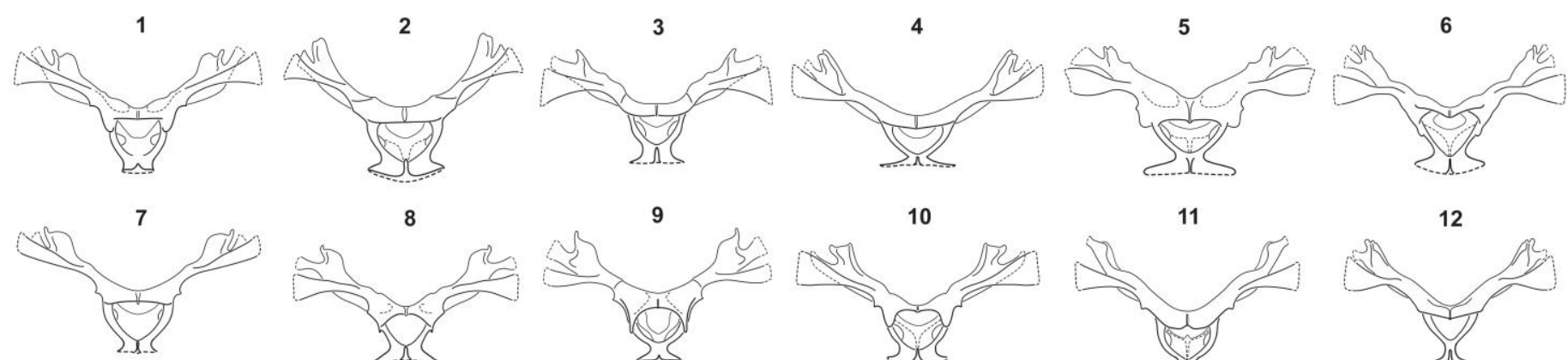

8

9

10

11
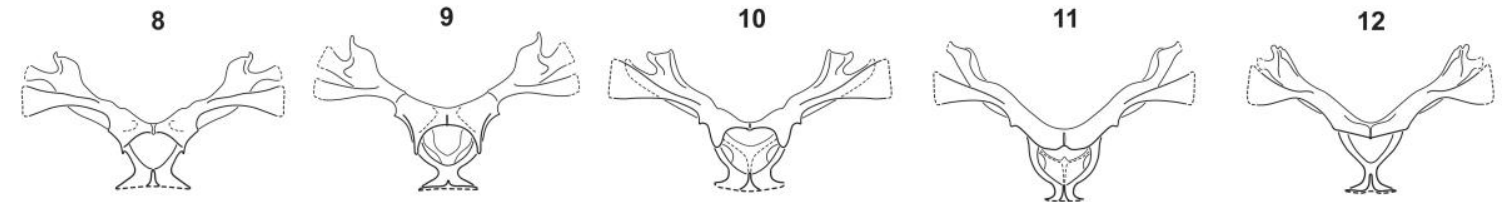

13

14

15

16

17

18
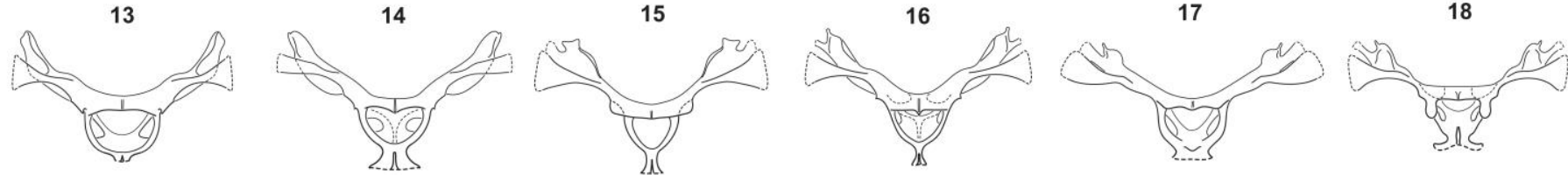

19
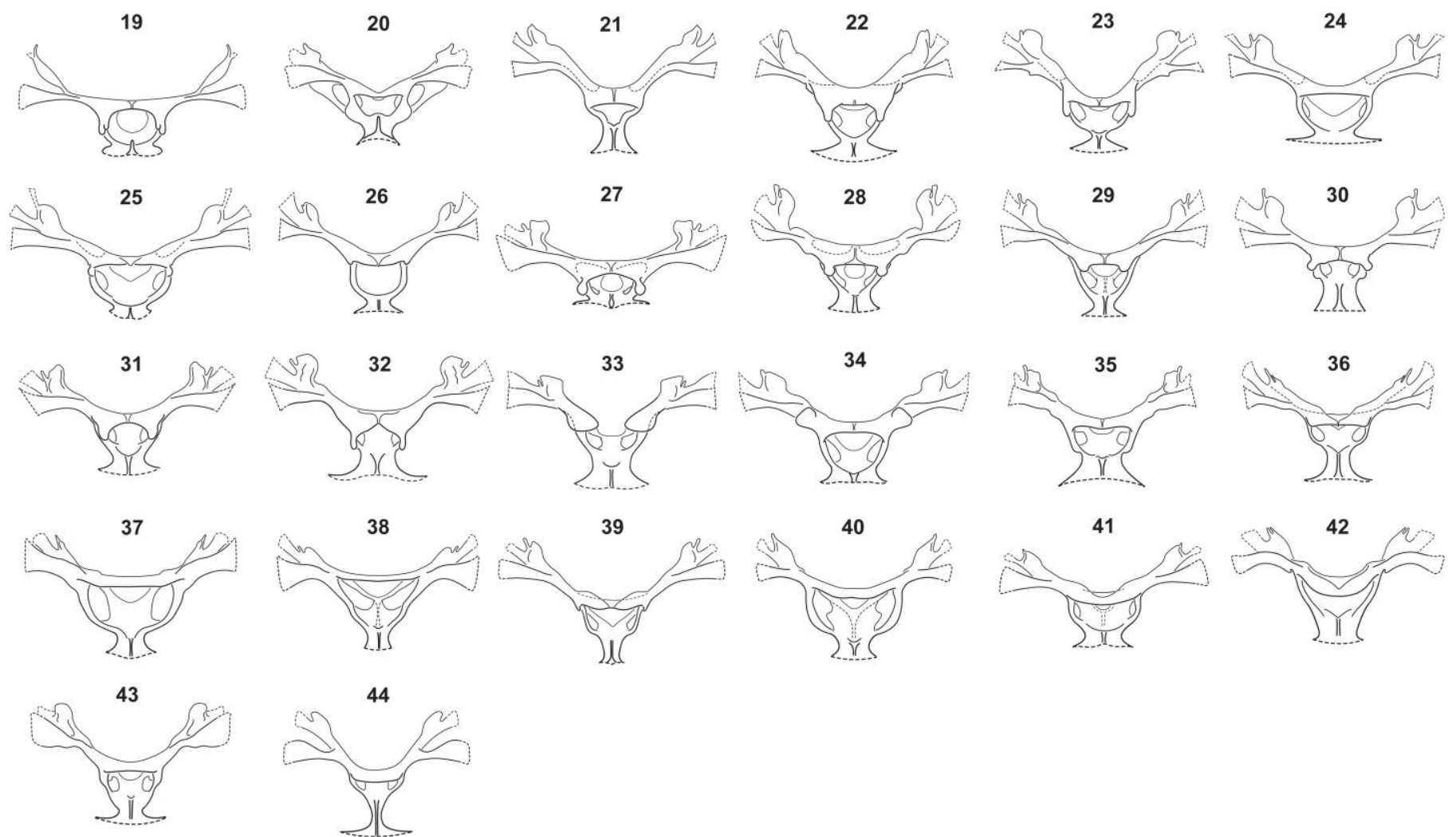

Figure 10. Mesofurca/metafurca; anterior view; reference numbers for taxa as in Table 1. 

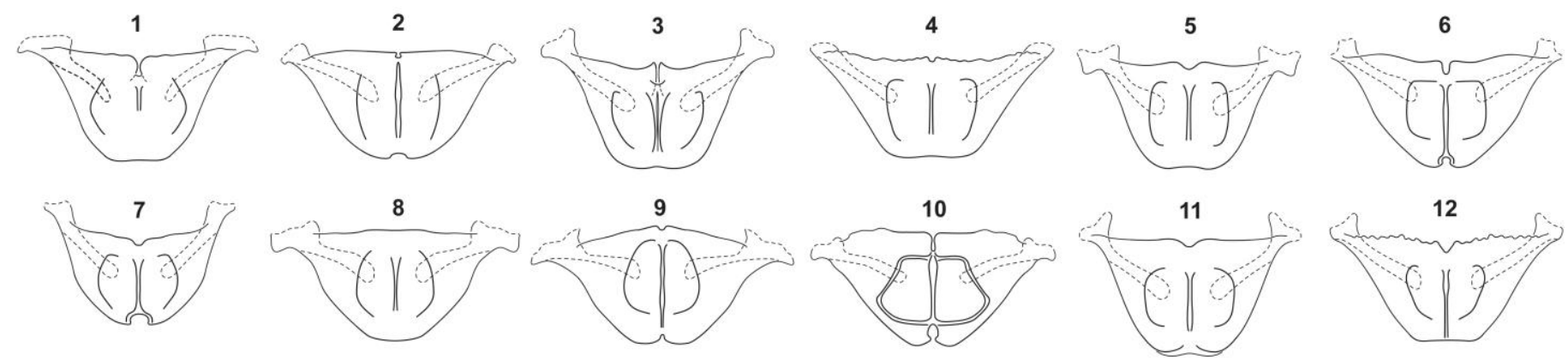

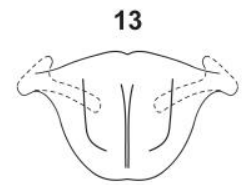

19
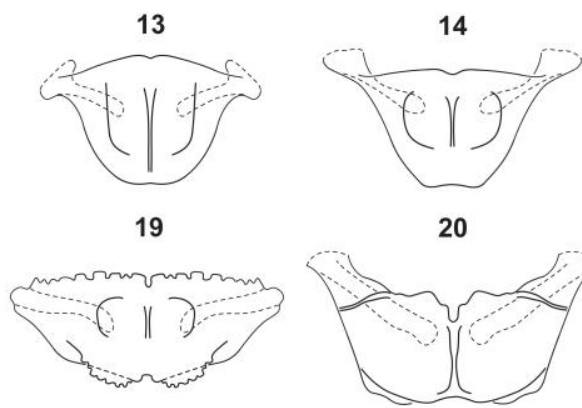

20

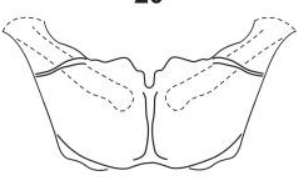

26
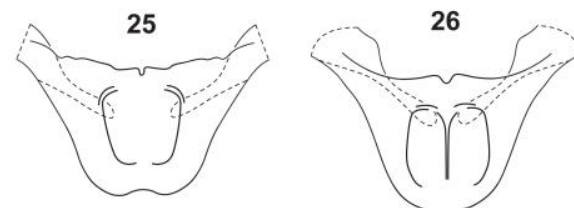

32
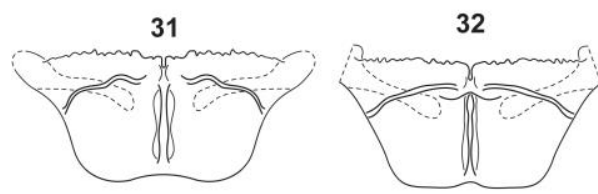

39
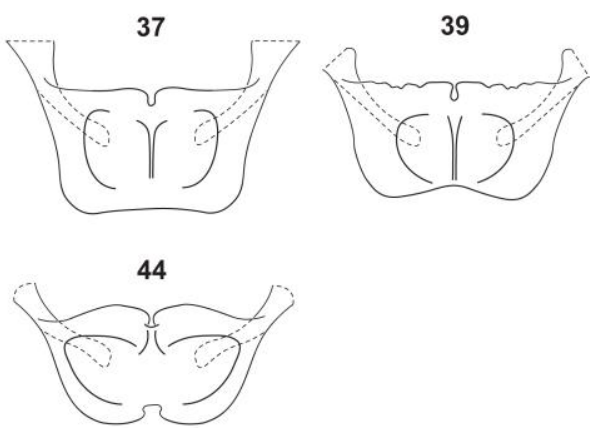

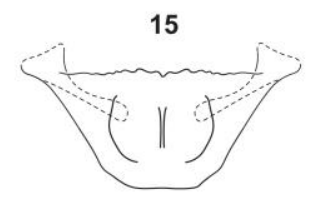

21

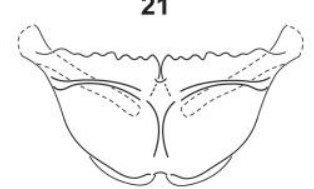

27
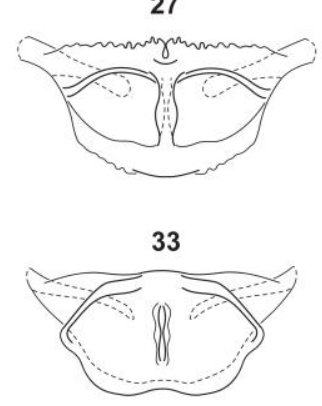

40

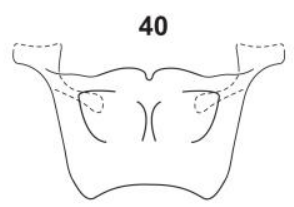

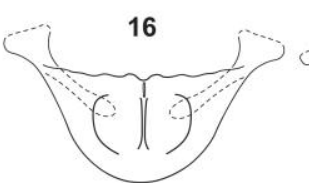

22

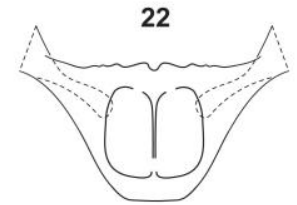

28
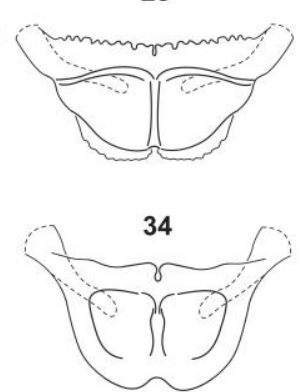

41

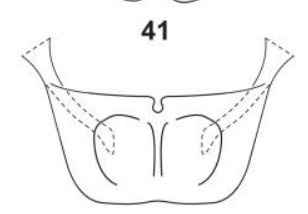

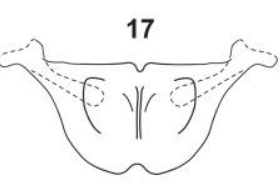

23

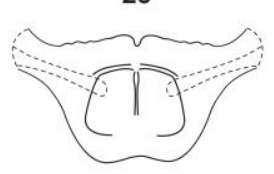

29
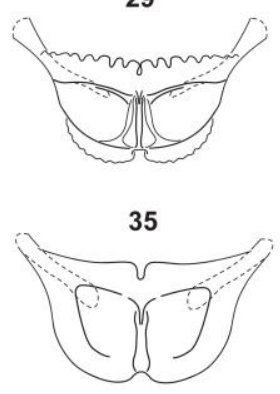

42

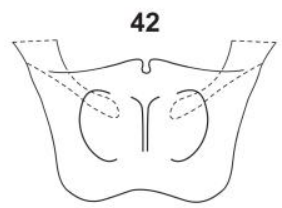

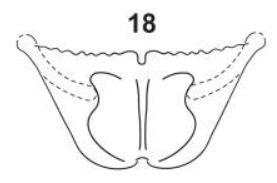

24

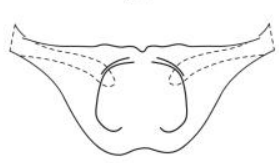

30
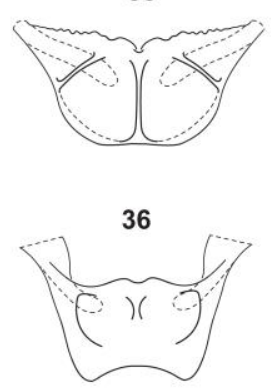

43

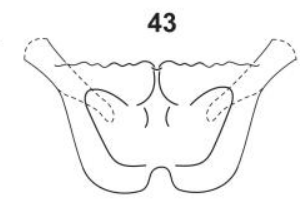

Figure 11. Mesophragma; posterior view; reference numbers for taxa as in Table 1. 
Figure 12. (next page) Mesosomal character state changes on a phylogenetic hypothesis for bees. Top: tree based on Danforth et al. (2013). Bottom (box): trees representing the corbiculate relationships under two different scenarios (I and II). Scenario I depicts most trees obtained from molecular datasets. Scenario II is a summary of the trees obtained from the majority of phenotypic (morphological and behavioral) datasets. In both scenarios, the internal relationships of Euglossina and Meliponina follow the phylogenies published by Ramírez et al. (2010) and Rasmussen \& Cameron (2010), respectively. For more details on the different phylogenetic hypothesis for corbiculate bees, see review by Almeida \& Porto (2014). Filled shapes represent unique changes; open shapes represent homoplasious changes (multiple independent transformations as well as reversed transformations); values above shapes are character numbers and values below shapes are the character states changes; asterisks indicate those characters state changes present only in scenario I. Symbols code: circles = prosternum; squares = propleuron; triangles = meso/metafurca; stars = mesophragma. 


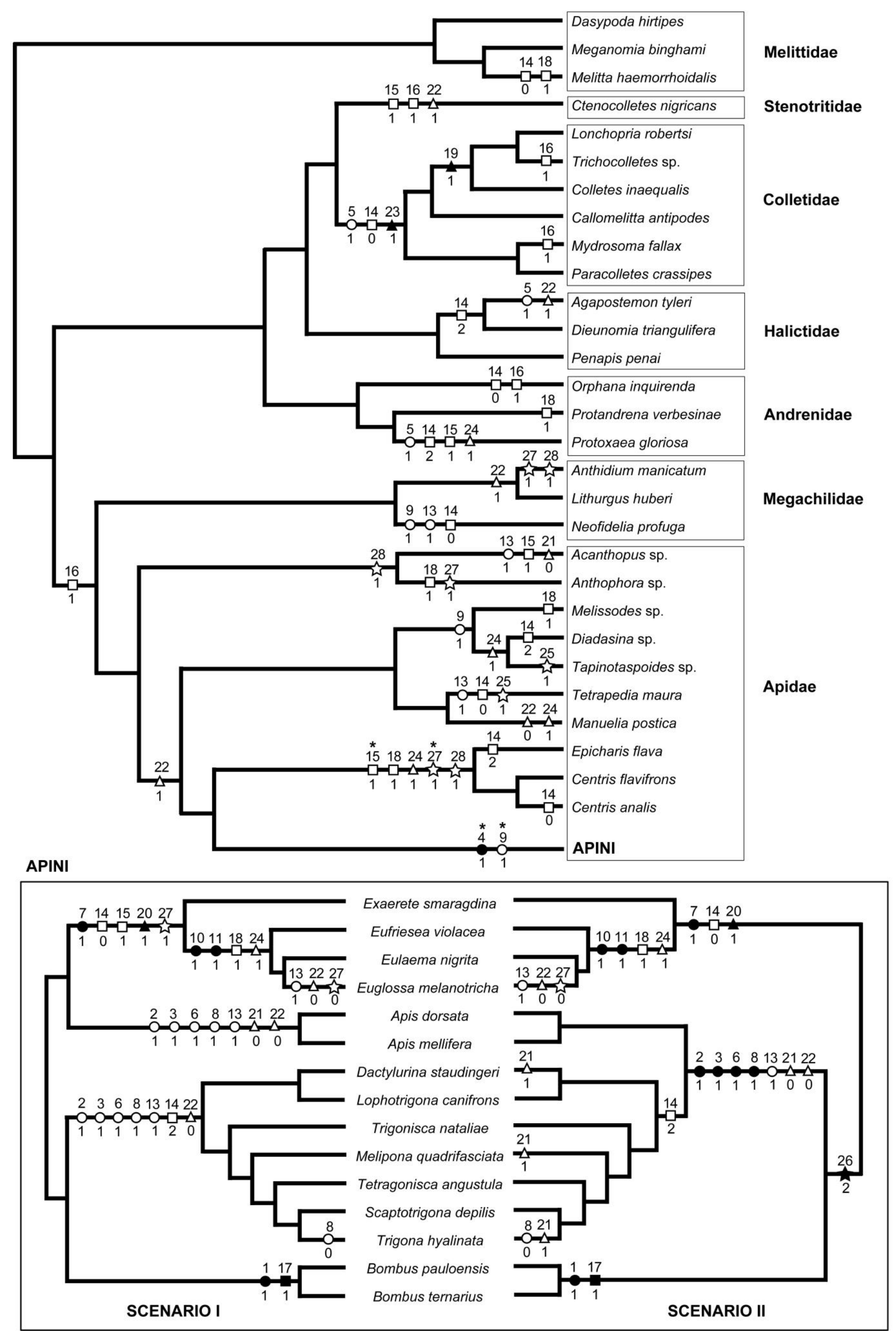




\title{
Chapter 3 - Phylogenetic analysis of corbiculate bees (Hymenoptera:
}

\section{Apidae: Apini) inferred with new data from internal structures of exoskeleton}

\begin{abstract}
Corbiculate bees are a well-known group of Apidae whose members exhibit a broad spectrum of social behaviors, including eusociality. The longstanding controversy concerning its phylogenetic relationships is a main critical issue to the understanding of the evolution of eusociality. A set of different phylogenetic analysis including the main lineages of Apidae with emphasis in the corbiculate bees is conducted in this study. A morphological matrix with 93 character from external morphology and 42 from internal structures of exoskeleton was constructed and the preferred analysis resulted into two most parsimonious trees with 376 steps, $\mathrm{CI}=45$ and $\mathrm{RI}=78$. The results presented are congruent with the current morphological/behavioral scenario for the corbiculate relationships: Euglossina $+($ Bombina $+($ Apina + Meliponina $)$ ). Also, an alternative analysis was conducted to cope with continuously varying characters "as such". It is demonstrated that characters extracted from internal structures of exoskeleton (e.g., phragma, furcae, apodemes), although poorly explored, can provide critical evidence to elucidate the "corbiculate controversy", since they offered 18 unique derived character states supporting groupings into the corbiculate clade.
\end{abstract}

\section{Introduction}

Honeybees (Apina), bumblebees (Bombina), orchid bees (Euglossina), and stingless bees (Meliponina) comprise the well-known clade of the corbiculate bees (Apidae: Apini), which approximately 1,000 valid species, almost half of this described only for the South America (Ascher \& Pickering, 2014). This group has attracted interest of many biologists for centuries due to notable ethological, physiological and morphological aspects, particularly those of the honeybees (von Frisch, 1946; Snodgrass, 1910), and most recently mainly because two important issues: the astonishing diversity of distinct "kinds" of social behaviors exhibited through its representatives - solitary, communal, "totipotent-caste" and "fixed-caste" eusocial species (Almeida \& Porto, 2014) - and the longstanding controversy concerning its phylogenetic relationships (Schultz et al., 
1999; Cardinal \& Packer, 2007; Kawakita et al., 2008; Cardinal \& Danforth, 2011). Advances on the study of social behaviors were achieved primarily due to a growing body of information about the social organization of representatives of the stingless bees (Kerr, 1969, 1987; Michener, 1974; Sakagami, 1982; Engels \& Imperatriz-Fonseca, 1990; Imperatriz-Fonseca \& Zucchi, 1995; Faustino et al., 2002; Tarpy \& Gilley, 2004; Tóth et al., 2004; Santos-Filho et al., 2006) and orchid bees (Garófalo et al., 1998; Soucy et al., 2003; Cameron, 2004; Augusto \& Garófalo, 2009, 2011). The corbiculate phylogeny still are, however, an open matter despite the efforts dedicated in the last 70 years since the pioneering work of Michener (1944). This is certainly a critical issue because a reliable phylogenetic background is desirable and necessary for accurate interpretation of the evolution of social traits in this group (Noll, 2002; Cardinal \& Danforth, 2011; Almeida \& Porto, 2014).

Discrepancies have grown even bigger because the increasing amounts of molecular data recover phylogenies incongruent with many morphology-based hypotheses published so far (Sheppard \& McPheron, 1991; Cameron, 1991, 1993; Mardulyn \& Cameron, 1999; Schultz et al., 1999; Cameron \& Mardulyn, 2001; Kawakita et al., 2008; Almeida \& Porto, 2014). The present scenario is one in which the majority of the hypotheses are divided into two main groups: phylogenies with Meliponina as the sister group of Bombina (molecular datasets: e.g., Sheppard \& McPheron, 1991; Cameron, 1991,1993, Koulianos et al, 1999; Mardulyn \& Cameron, 1999; Cameron \& Mardulyn, 2001; Kawakita et al., 2008; Cardinal et al., 2010; Cardinal \& Danforth, 2011, Cardinal \& Danforth 2013; Martins et al, 2014), and phylogenies with Meliponina as the sister group of Apina (most morphological and behavioral datasets: e.g., Michener, 1944, 1974, 1990; Maa, 1953; Prentice, 1991; Roig-Alsina \& Michener, 1993; Chavarría \& Carpenter, 1994; Schultz et al., 1999; Ascher et al., 2001, Engel, 2001a, b; Noll, 2002; Cardinal \& Packer, 2007; Payne, 2013; Canevazzi \& Noll, 2015). The sources of this strong discordance are unknown and largely debated in literature, and then some alternatives to solve this issue were suggested: "total evidence" analysis (Chavarría \& Carpenter, 1994; Schultz et al., 1999; Cameron \& Mardulyn, 2001; Ascher et al., 2001; Payne, 2013; Canevazzi \& Noll, 2015); inclusion of more molecular (Kawakita et al., 2008) and/or morphological (RoigAlsina \& Michener, 1993; Engel, 2001a,b; Cardinal \& Packer, 2007) data; inclusion of fossil taxa (Engel, 2001a, b); and alternative analytical methodologies (Payne, 2013). Whereas a considerable part of these efforts were concentrated on the expansion of 
molecular datasets (e.g., Kawakita et al., 2008), morphology - with exception of the work of Cardinal \& Packer (2007) - has been relatively neglected in the last 10 years, since the work of Roig-Alsina \& Michener (1993), which have added a substantial quantity of new data - especially from internal skeletal structures.

In this paper, a phylogenetic analysis of the corbiculate clade is made constructing a morphological dataset including almost all relevant characters proposed by previous authors and adding new information from head, mandibles, sitophore, propectus, mesofurca/metafurca and mesophragma. The trees obtained were then contrasted with the main concurrent scenario for the relationships of this tribe. It is demonstrated that characters extracted from internal structures of exoskeleton (e.g., phragma, furcae, apodemes), although poorly explored, present phylogenetic signal and that can become substantially relevant for other phylogenetic studies in bees.

\section{Material and methods}

\section{Taxon sampling}

Representatives of 25 species pertaining to the family Apidae were chosen for this study - 15 encompassing Apini and 10 outgroup taxa amongst 8 other tribes (Table 1). The sampling attempted to include representatives of all major lineages of Apini as recovered in recent phylogenetic hypotheses (Alexander, 1991a, b; Hines, 2008; Ramírez et al., 2010; Rasmussen \& Cameron, 2010). As indicated by most morphological and molecular studies including this tribe (Roig-Alsina \& Michener, 1993; Cardinal et al., 2010; Cardinal \& Danforth, 2011; Cardinal \& Danforth, 2013), Centridini is the likely sister-group of corbiculate bees, thus two representatives of Centris and one of Epicharis were selected. All the specimens examined in this paper are deposited in the "Coleção Entomológica J.M.F. Camargo (RPSP)", Departamento de Biologia, FFCLRP-USP. The classification of bee taxa followed Michener (2007) and the status of corbiculate bees as subtribes followed the suggestion of A. Roig-Alsina (in Roig-Alsina \& Michener, 1993; p. 158) and also adopted by Silveira et al. (2002).

\section{Character construction and coding}

For character construction, only females of the representative taxa presented in Table 1 were used. In the case of eusocial species (e.g., Apina, Bombina, and Meliponina), only workers were examined. All the characters proposed by previous authors and used in 
this work were coded for the taxa sampled for this study, as shown in Table 1. When new interpretations for the character state delimitations and/or coding of taxa were done, these decisions were clarified on the character comments. In total, 135 characters were constructed for this study, 93 from external morphology and 42 from internal skeletal structures; 51 new characters or reinterpretations of characters already used from head, mandibles, sitophore, propectus, mesofurca/metafurca and mesophragma were added to the existing morphological datasets for bees. For the characters encompassing ratios between structures or dimensions, measures were taken directly from the specimens through an optical micrometer. The tilt angles of propleural arms (char. 110) were measured using a protractor on line drawings of the respective structures. Character-states describing continuous variation were constructed considering the gaps observed to exist between the classes of values (i.e., depicted as states), although in some instances the exact limits of states might have been defined arbitrarily. The commented character list used in this work is given in Appendix 1 and the matrix of character state distribution in Table 2.

\section{Phylogenetic methods}

Parsimony analyses were executed using the procedures implemented in TNT v1.1 software (Goloboff et al., 2008). Tree searching was performed with the traditional search option, with random seed sat as 0 and 1000 replications, under equal weights (EW). All the characters were treated as nonadditive. The characters encompassing continuous variation, but coded as discrete multistate, where indicated with stars in the character list in Appendix 1. Three sets of analyses (A-C) were performed to explore the morphological dataset. The first set (A), included the full matrix with all taxa and characters. The second set (B), included a reduced matrix with all the characters but without the cleptoparasitic taxon Acanthopus sp. The third set (C), included the matrix used in B, but without the continuous characters (stars on Appendix 1). For two of the three sets of analyses (B-C), additional tree searches were conducted using implied weighting (IW) to evaluate how the morphological data characters behave under differential weighting schemes (i.e., $\mathrm{k}$ values) against the homoplasious changes (Goloboff, 1993). To account for possible arbitrary decisions in defining some of the intervals used to construct the states of the continuous characters in analysis B, an alternative separate analysis (D) utilizing the continuous data "as such" was conducted in TNT (as detailed by Goloboff et al., 2006). The values of the measures used in the continuous characters are given on Appendix 2. Some of the 
continuous characters treated as discrete, were not included in analysis D and were indicated with double stars on Appendix 1. Transformations of character states were accessed with Fitch optimization and mapping schemes in the software WinClada (Nixon, 2002). The trees obtained were rooted between Antophorini and the remainder taxa, using Antophora sp. as outgroup according to recent views on phylogenetic relationships of Apidae (Cardinal et al., 2010; Cardinal \& Danforth, 2011, 2013; Danforth et al., 2013). The branch support was estimated calculating the Bremer support values for each branch (Bremer, 1994), and complemented with the calculation of standard bootstrapping and non-parametric bootstrap (Felsenstein, 1985) with symmetric resampling (Goloboff et al., 2003).

\section{Results}

The first set of analysis with the full dataset (A) resulted into four most parsimonious trees (Fig. 1, A: T1-T4) with 405 steps (L), consistency index 42 (CI) and retention index $76(\mathrm{RI})$, whose strict consensus is depicted in Figure 1 (A, leftmost tree). Although the relationships between the major linages of Apidae and monophyly of the majority of its main clades are recovered in all the equally most parsimonious trees obtained (T1-T4) - Centridini, Apini, Euglossina, Bombina, Apina, Meliponina accordingly with the majority of morphological (Michener, 1990; Prentice, 1991; RoigAlsina \& Michener, 1993; Chavarría \& Carpenter, 1994; Engel, 2001a, b), behavioral (Noll, 2002; Canevazzi \& Noll, 2015) and molecular studies (Kawakita et al., 2008; Cardinal et al., 2010; Cardinal \& Danforth, 2011; Cardinal \& Danforth, 2013; Martins et al., 2014), the varying positioning of the cleptoparasitic taxon Acanthopus sp. in these trees resulted into the disruption of well-supported clades commonly recovered in those studies - as the grouping of Centridini + Apini. This can be viewed as an artifact caused by morphological changes associated with the cleptoparasitic habit producing a long morphological branch separating this taxon from the remainder Apidae (for a more extensive discussion of this matter, refer to Roig-Alsina \& Michener, 1993). This morphological disparity can facilitate spurious groupings of Acanthopus sp. with different linages - "Eucerine-Lines", Centridini or Apini - simply due to homoplasy. An alternative interpretation is that the present dataset just does not contain sufficient character information to gives an accurate placement for Acanthopus sp. in the strict consensus tree. Thus, considering that the main objective of this work is not to solve the relationships 
between the main linages of Apidae nor recover the proper placement of Ericrocidini amongst the other Apinae, another set of analysis were conducted trying to avoid the disturbance caused by the presence of Acanthopus sp.

The second set of analyses with Acanthopus sp. removed (B) resulted into two most parsimonious trees with 376 steps (L), consistency index $45(\mathrm{CI})$ and retention index 78 (RI). The strict consensus is showed in Figure 1 (B, leftmost tree). The different trees obtained with the analyses using implied weighting (IW) are exhibited in the same figure ( $\mathrm{B}$, trees to the right), with the respective $\mathrm{k}$ values chosen. The relationships of the strict consensus tree were fully resolved with exception of the tricotomy of Tapinotaspoides sp., Tetrapedia maura and Manuelia postica. With the removal of the rogue taxon Acanthopus sp., the monophyly of Centridini + Apini was recovered. The different trees obtained from the cladistic analyses with implied weighting (IW) presented topologies mostly congruent with the strict consensus under equal weights. In all the implied weighting trees the tricotomy present in the equal weights consensus was resolved according to the following: (Tapinotaspoides sp. (Tetrapedia maura + Manuelia postica)). The only differences in the tree topologies concern the positioning of the Old-World meliponine taxa. In the equal weights consensus tree and implied weighting trees with $\mathrm{k}$ values above 5, Dactylurina staudingeri and Lophotrigona canifrons do not group with the remainder Neotropical meliponine clade (c.f., Rasmussen et al., 2010). In the implied weighting trees with $\mathrm{k}$ values bellow 5, Dactylurina and Lophotrigona nest inside the latter.

Lockhart \& Cameron (2001) have suggested the use of continuous characters coded discretely as a possible source of disturbance leading to incongruence between morphological and molecular topologies. To evaluate the influence of the continuous characters on the trees recovered, a third set of analyses (C) with Acanthopus sp. removed and a matrix without continuous data was conducted. It resulted into one most parsimonious tree (Figure 1, C, leftmost tree) with 246 steps (L), consistency index 50 (CI) and retention index 81 (RI). Different trees obtained with implied weighting analyses (IW) are shown in the same figure accompanied by the respective $\mathrm{k}$ values $(\mathrm{C}$, trees to the right). The tree topology obtained in the strict consensus was almost completely concordant with the one from the previous analysis, with exception of the complete resolution of the tricotomy between Tapinotaspoides sp., Tetrapedia maura and Manuelia postica; the positioning of Eufriesea violacea; and the grouping of the Old-World meliponine taxa. The implied weighting analyses with different $\mathrm{k}$ values resulted into trees whose topologies 
were mostly similar to the one of the consensus, excepting for the non-monophyly on Centridini + Apini in some of them $-\mathrm{k}$ values bellow 10. Also, the positioning of Manuelia postica, Euglossa melanotricha and Dactylurina staudingeri were variable inside their respective more inclusive clades.

The alternative analysis (D) with the continuous data treated "as such", following the proposal of Goloboff et al. (2006), resulted in the tree presented in Figure 2. The tribal and subtribal relationships of the taxa are in great concordance with the results presented in the strict consensuses of analyses B and C. The main differences are in the internal relationships of Meliponina. In this tree, the clade formed by Melipona quadrifasciata + Scaptotrigona depilis is surprisingly placed as sister to the remainder Meliponina, thus disrupting the Neotropical clade.

Taking into account that the strict consensus trees obtained in the analyses B-C and in the alternative analysis D were concordant in the majority of the relationships depicted, the EW tree resultant from analysis B will be chosen for the following discussions (Figure 3). Although somehow arbitrary, this choice relates to the fact that analysis B contains the most complete morphological dataset without the disturbances caused by the rogue taxa Acanthopus sp. in addition to a more conservative way of dealing with continuous characters. The non-parametric bootstrap and Bremer support values were showed in Table 3. Although the main interest of this work is the relationships of corbiculate taxa, few comments deserve to be made on the outgroups as well.

\section{Discussion}

\section{Phylogenetic relationships of the main lineages of Apidae}

Considering the tree shown in Figure 3, the clade indicated by node 1 was also recovered in all the other trees resultant from the analyses conducted in this work (A-D). The monophyly of the taxa above node 1 is supported by three uncontroverted and six homoplasious character state changes. The indistinct subgaleal sclerite (char. 30:0), relatively elongated paraglossae (char. 45:2) and more or less parallel ridges on the posterior surface of mesophragma (char. 130:0) are uncontroverted synapomorphies for this clade. Furthermore, weakly produced paramandibular processes (char. 104:1) without a carina (char. 105:1) and fully sclerotic entire anterodorsal margin of mesophragma (char. 131:0), although representing homoplasious changes, are additional evidence for this grouping. The clade including Tetrapedia spp. and representatives of the so called 
“Eucerine line" - Melissodes sp., Diadasina sp., Tapinotaspoides sp. - and Xylocopinae Manuelia postica - is congruent with most results obtained from molecular datasets (Cardinal et al., 2010; Cardinal \& Danforth, 2011, 2013; Martins et al., 2014).

The grouping of Melissodes sp. and Diadasina sp. (node 2) is recovered in almost all analyses conducted (B-D). In Figure 3, it is supported by four homoplasious changes, including the absence of bristles on the surface of furcasternum (char. 120:0), though this condition is also found in the corbiculate clade (node 6). The close relationship of Melissodes sp., Diadasina sp. and Tapinotaspoides sp. has already been recovered with morphological data (Roig-Alsina \& Michener, 1993) and molecular data (Cardinal et al., 2010; Cardinal \& Danforth, 2011, 2013; Martins et al., 2014), but the grouping recovered in node 2 has never been found before. Roig-Alsina \& Michener (1993) have found Melissodes spp. sister to Tapinotaspoides spp., whereas most molecular datasets (e.g., Cardinal et al., 2010) recover Diadasina spp. sister to Tapinotaspoides spp. In this work, Tapinotaspoides sp. was placed into a tricotomy with Tetrapedia maura and Manuelia postica in the strict consensus tree of analysis $\mathrm{B}$. The node 3 is supported by five homoplasious and two uncontroverted character state changes. The uncontroverted changes include the horizontal configuration of the metanotum in profile (char. 53:0) and ratio of width to high of metepisternum (char. 54:0). The reduction of the fan-shaped sheets of the posterior tentorial arms (char. 98:0) is another evidence from internal head morphology that adds to the evidence supporting this node, although have convergently appeared in Exaerete smaragdina and the clade of Apina + Meliponina (node 11). Despite the inclusion of Tapinotaspoides sp. in the clade indicate by node 3, the grouping of Manuelia postica and Tetrapedia maura has been recovered in almost all molecular datasets (Cardinal et al., 2010; Cardinal \& Danforth, 2011, 2013).

The monophyly of Centridini + Apini (node 4) is recovered in all the trees of analyses B and D and some of the C. In the chosen tree, it is supported by four unequivocal character state changes: presence of two teeth on pollex (char. 17:1), two or less segments in the maxillary palpus (char. 37:4), ratio of width to height of metepisternum (char. 54:2), and broadened posterior basitarsus (char. 88:1). This result is totally congruent with almost all major previous studies with morphological and molecular datasets (Roig-Alsina \& Michener, 1993; Cardinal et al., 2010; Cardinal \& Danforth, 2011, 2013; Martins et al., 2014). The monophyly of Centridini (node 5) is a sensitive issue, once it has been commonly recovered with morphology (Roig-Alsina \& Michener, 1993), but not with 
molecules (Cardinal et al., 2010; Cardinal \& Danforth, 2011; Martins et al., 2014). In this work, the monophyly of Centridini is provided by three homoplasious changes and two uncontroverted synapomorphies, the latter including the simple toothed tarsal claws present only in the posterior legs (char. 91:1) and third phragma terminating at the marginal area of propodeum (char. 135:1).

\section{Phylogenetic relationships of corbiculate bees}

The monophyly of corbiculate bees (Apidae: Apini) is practically universally accepted and recovered in all morphological, behavioral, and molecular phylogenies proposed by previous authors (Cardinal et al., 2010; Noll, 2002; Cardinal \& Danforth; 2011, 2013; Martins et al., 2014). In this work, the monophyly of Apini is found in all the trees of the analyses executed (A-D). Six unequivocal and three homoplasious changes yield the monophyly of this clade. The uncontroverted changes includes: spatulate mandibular apex (char. 15:1); posteriorly projected mesoscutellum (char. 51:1); presence of sclerotic bars on the area between the metacoxae and first metasomal sternum (char. 56:1), absence of basitibial plate (char. 76:2); proximal basitarsal articulation displaced from the dorsal margin of metatibia (char. 86:1) and a shallow or absent profurcal pit (char. 116:1). Also, the smooth surface of furcasternum (char. 120:1) constitutes additional evidence from the propectus, although this condition has appeared convergently in node 2. When considering the external surface of the metatibia modified into a corbicula (char. 77:1), and the presence of the rastellum on the apex of metatibia (char. 84:1), two additional synapomorphies can be added to this list (these transformations are not shown on Figure 3 because they were not unambiguously optimized onto this tree).

The monophyly of each subtribe of Apini has been found in all trees and all analyses conducted in this work (A-D). The naturalness of these groupings have not been seriously questioned in any phylogenetic hypotheses published so far, independently of the source of data used (Michener, 1990; Prentice, 1991; Roig-Alsina \& Michener, 1993; Chavarría \& Carpenter, 1994; Schultz et al., 1999; Engel, 2001a, b; Cardinal et al., 2010; Cardinal \& Danforth, 2011, 2013; Martins, et al., 2014; Canevazzi \& Noll, 2015). Only the work of Noll (2002) has not recovered the monophyly of Euglossina, but this was based solely on behavioral data.

Euglossina (node 7) is supported by seven unequivocal character state changes: lower portion of epistomal sulcus strongly bent laterally (char. 5:1); deep postoccipital 
pouch (char. 12:1); basal margin of submentum profoundly cleft (char. 40:1); reduced jugal lobe on posterior wings (char. 67:0); presence of lateral spurs projecting from the dorsal sheet of anterior tentorial arm (char. 93:1); surface of anterior process of prosternum with distinct transversal folding lines (char. 118:1); and deeply emarginated anterior margin of mesofurcal bridge (char. 125:1). The grouping of the non-cleptoparasitic Euglossina included in this study (node 8 ) is supported by the presence of a double median profurcal lamella (char. 121:1) and the development of internal cuticular expansions on the posterodorsal profurcal apodemes (char. 122:1). Taking into account that Aglae caerulea Lepeletier \& Serville, 1825 is not sampled in the present study, the grouping of Exaerete smaragdina sister to clade represented by node 8 is congruent with the molecular phylogenetic hypotheses of Michel-Salzat et al. (2004) and Ramírez et al., (2010). Also, the clade composed by Euglossa melanotricha and Eufriesea violacea is congruent with the work of Engel (1999) with fossil taxa and the hypothesis based on the sting matrix of Cardinal \& Packer (2007), although in the latter work the position of species of Eulaema were not shown in their Fig. 1. Bombina (node 10), in its turn, is supported by three uncontroverted changes in the tree of Figure 3: absence of jugal lobe (char. 66:1); strongly emarginated anterior margin of ventral propleural area (char. 112:1) and conspicuously broadened anterior process of prosternum (char. 113:1).

The clade comprising Bombina, Apina, and Meliponina (node 9) present nine synapomorphies, seven of them being uncontroverted changes: antennal foramina closely approximated (char. 2:0); pollex teeth reduced to less than half the breadth of the tooth of rutellum (char. 18:1); separated mentum and submentum (char. 39:1); ventrally arched tentorial bridge (char. 99:1); short hypopharyngeal lobe (char. 108:1) with trilobate apex (char. 109:2); and the arched ridges on the posterior surface of mesophragma (char. 130:2). The monophyly of this clade is supported by three characters prevenient from internal skeletal morphology (i.e., sitophore, tentorial bridge and mesophragma), but there also are character state transformations related to ratios/measures between structures or possibly influenced by ecological/developmental constraints (chars. 2, 18, 108) supporting this clade. This result is completely concordant with most morphological and behavioral hypotheses published to the present (Prentice, 1991; Roig-Alsina \& Michener, 1993; Chavarría \& Carpenter, 1994; Engel, 2001 a, b; Noll, 2002). One important point to be emphasized here is that there is no morphological synapomorphy in the knowledge of the authors that was proposed so far corroborating unequivocally a clade of Euglossina with 
Apina, as often recovered with molecular sequences (Cardinal et al., 2010; Cardinal \& Danforth, 2011, 2013; Martins et al, 2014).

The clade Apina + Meliponina (node 11) is supported by more than ten unique derived character states: low positioning of antennal foramina (char. 1:1); absence of condylar (char. 20:1) and outer grooves of mandibles (char. 21:1); flattened antero-lateral processes of mesoscutum (char. 46:1); transversally oriented first recurrent vein on anterior wings (char. 61:1), absence of spines on the dorsal margin of malus (char. 71:1); absence of metatibial spurs (char. 80:1); strongly produced lateral processes of prosternum (char. 114:1); absence of profurcal pit (char. 115:1); constricted connection of basisternum to furcasternum (char. 117:1); and the surface of basisternum without bristles (char. 119:1). As it was done before for node 9 , if the continuously varying data and characters possibly correlated to other environmental factors - as chars. 1, 20, 21 - and losses/reductions/absence - as chars. 71, 80, 119 - were excluded, the monophyly of this clade is still maintained by four unequivocal changes.

Apina (node 12) is supported by seven homoplasious and two uncontroverted changes, the latter two being: absence of acetabular grooves on mandibles (char. 19:1) and the distinctively broadened subgaleal sclerite (char. 31:1). Meliponina (node 13) is supported by 10 unique derived character states: presence of oblique grooves on mandibles (char. 23:1); distal portion of subgaleal sclerite strongly bent laterally (char. 32:1); only one segment in the maxillary palpus (char. 37:5) with a more or less quadrangular shape (char. 38:0); absence of the supra-alar carina (char. 50:1); opened marginal cell on forewing (char. 58:1); presence of penicillum on the distal margin of metatibia (char. 85:1); all tarsal claws simple toothed (char. 90:1); displaced insertion of frontal muscles on pharyngeal rods (char. 107:1); and much reduced third phragma (char. 134:0). The Neotropical-clade of Meliponina (node 14, c.f. Rasmussen et al., 2010) is supported only by two homoplasious transformations related to the shape of the marginal cell on the forewing (char. 57:1) and the attachment of the thickening of secondary tentorial bridge to the internal surface of head capsule closing the space beneath the antennal foramen (char. 94:2). Despite the fact the Neotropical clade has been recovered in the strict consensus tree of analysis A, all the trees in analysis C; and some trees of analysis B; and the Old World taxa have grouped into a clade in the strict consensus tree of analysis $\mathrm{C}$ - accordingly with the results of Rasmussen et al. (2010) - the remainder internal relationships of Meliponina were not in concordance with the main previous works based on morphology (Michener, 
1990; Camargo \& Pedro, 1992). It is important to emphasize, that the results presented for Meliponina should be taken with caution, especially considering that most of the internal groupings are sustained by homoplasious changes associated with continuously varying characters. The only unequivocal change supporting a clade is the acute posterior angle of corbicula (char. 79:2), which groups Scaptotrigona depilis, Trigonisca nataliae, Melipona quadrifasciata and Trigona hyalinata. However, as the main interest of this work was centered in the subtribal relationships of Apini, the search for derived character states was focused on that level. Thus, with the inclusion of more morphological data specifically to address the internal relationships of Meliponina, this scenario probably would change.

\section{Evaluating alternative scenarios for the phylogenetic relationships of corbiculate bees}

If the morphological matrix constructed for this work is reduced to contain only the changes informative for corbiculate bees and then optimized onto the phylogenetic hypotheses commonly recovered in molecular studies (Figure 4, B-C) in contrast with that obtained here (Figure 4, A), some comments can be made. Only two unequivocal character state changes would support Apina and Euglossina as a clade (Fig. 4, B): the relatively elongate first recurrent vein on anterior wing (char. 60:2), though it is associated with a continuously varying measure, and the strongly developed carina produced into a spine on the anterior margin of velum (char. 73:2). Additionally, at least five uncontroverted synapomorphies would favor the grouping of Meliponina with Bombina: reduced teeth of pollex (char. 18:1); densely pubescent internal surface of laciniae (char. 34:0); conjunctive bonding the posterior portion of metasternum to the first metasomal sternum arising at the distal margin of metasternum (char. 55:1); wide area of fusion of hypostoma to the secondary tentorial bridge (char. 101:1); and trilobate apex of hypopharyngeal lobe (char. 109:2). Even if the absence of the pollex teeth in Apina (char. 16: 0) is considered an extreme reduction of the teeth, there still are three unique derived character states for this clade in this scenario. On the other hand, if the reduced matrix is optimized onto the EW tree recovered in analysis B considering only the corbiculate taxa, no less than 15 unique changes favor the clade Bombina-Apina-Meliponina and more than 20 provide evidence for the clade Apina-Meliponina. Considering also the ambiguous optimizations resolved with the DELTRAN scheme, these numbers would rise to 17 derived character state changes for the former and more than 35 for the latter. The main sources of unequivocal evidence favoring the grouping of Apina and Meliponina are prevenient from the head 
capsule (external and internal), mandibles/mouthparts, wings and prosternum, whereas the alternative grouping of Meliponina and Bombina is favored by one modification in the tentoria/hypostoma, two in mandible/mouthparts and one in the sitophore. It is interestingly to note that even in a scenario where Meliponina is sister to Bombina, the clade Bombina-Apina-Meliponina (Fig. 4, C), as found in a few works with molecular data (e.g., Cameron \& Mardulyn, 2001) is still supported by 14 unequivocal derived character states.

\section{Phylogenetic significance of internal characters}

The inclusion of more and new sources of data in addition to fossil taxa has been proposed as one alternative to solve the incongruences resulting in the "corbiculate controversy" (Chavarría \& Carpenter, 1994; Engel, 2001a, b). In this context, the input of new information from internal structures of exoskeleton is not only desirable, but also a necessity, since much of the external morphology of bees in general have already been exhaustively explored by previous authors, especially the mouthparts (e.g., Michener, 1984; Michener \& Brooks, 1984) and legs (e.g., Cane, 1979; Michener, 1981; Schönitzer, 1986). Few works have truly sought explore deeper into the internal structures for phylogenetic purpose (Roig-Alsina \& Michener, 1993; Melo, 1999). In this work from a total of 135 morphological characters, 42 were extracted from internal structures of exoskeleton, constituting approximately $31 \%$ of the morphological dataset. Although these numbers are certainly dependent on the way the characters are searched and chosen for a given work, it is notoriously the amount of data that can be obtained from these structures. From the total of 59 characters providing unequivocal evidence from the groupings depicted in nodes 1-14 in Figure 3, 20 were obtained from internal structures (about of $34 \%$ ). If only the corbiculate clade were considered, this number rises to around of $37 \%$. If these numbers are compared, for example, with the dataset of Roig-Alsina \& Michener (1993), from the total of 55 informative characters for corbiculate bees in that work (RoigAlsina \& Michener, 1993; Table 2, pg. 139), only 11 (20\%) were obtained from internal structures of exoskeleton. The data-matrix constructed in the present study, thus increased this number in about $17 \%$. Furthermore, internal structures have provided critical evidence concerning the "corbiculate controversy". The monophyly of Euglossina and the resolution of its internal relationships are supported by five characters from tentoria, prosternum and meso/metafurca. The grouping of Bombina, Apina, and Meliponina is evidenced by 
characters from tentoria, sitophore and mesophragma. Finally, the prosternum provides four unique derived character states as evidence for the clade uniting Apina and Meliponina. In this context, the exploration of internal morphology for character construction seems promising not only to address specific issues (e.g., "corbiculate controversy"), but also for future phylogenetic studies on bees. As can be seen in Figure 4, the CI and RI considering all the characters, only the external and only the internal ones are very similar, which corroborate their insertion as sources of morphological information. Also, it can be straightforward the employment of new technologies for phenotypic studies, since they are becoming increasingly accessible for comparative studies, tough still expensive to apply to large datasets (Deans et al., 2012).

\section{Acknowledgments}

This work was supported by the Master's Grant \# 2012/22261-8 and BEPE's Internship Grant \# 2014/10090-0 to D. S. Porto also by FAPESP, as well as by the grant \# 2011/09477-9, São Paulo Research Foundation (FAPESP) to the junior author. We are also very grateful to Lars B. Vilhelmsen by hosting the first author during the internship at the Zoological Museum in Copenhagen. We want also to acknowledge the help given by to Denise A. Alves, Sidnei Mateus, and Tulio Nunes with the meliponine material used in this study. The software TNT was made available through the sponsorship of the Willi Hennig Society and Kevin Nixon is acknowledged for making Winclada available. 
Table 1. List of the material examined in this study.

\begin{tabular}{|c|c|}
\hline Taxa & Species \\
\hline Apini & \\
\hline Apina & $\begin{array}{l}\text { Apis dorsata Fabricius, } 1793 \\
\text { Apis mellifera Linnaeus, } 1758\end{array}$ \\
\hline Bombina & $\begin{array}{l}\text { Bombus (Fervidobombus) pauloensis Friese, } 1913 \\
\text { Bombus (Pyrobombus) ternarius Say, } 1837\end{array}$ \\
\hline Euglossina & $\begin{array}{l}\text { Eufriesea violacea (Blanchard, 1840) } \\
\text { Euglossa (Euglossa) melanotricha Moure, } 1967 \\
\text { Eulaema (Apeulaema) nigrita Lepeletier, } 1841 \\
\text { Exaerete smaragdina (Guérin, 1844) }\end{array}$ \\
\hline Meliponina & $\begin{array}{l}\text { Lophotrigona canifrons (Smith, 1857) } \\
\text { Dactylurina staudingeri (Gribodo, 1893) } \\
\text { Melipona (Melipona) quadrifasciata Lepeletier, } 1836 \\
\text { Scaptotrigona depilis (Moure, 1942) } \\
\text { Tetragonisca angustula (Latreille, 1811) } \\
\text { Trigona hyalinata (Lepeletier, 1836) } \\
\text { Trigonisca (Trigonisca) nataliae (Moure, 1950) }\end{array}$ \\
\hline \multicolumn{2}{|l|}{ Outgroups } \\
\hline Anthophorini & Anthophora sp. \\
\hline Centridini & $\begin{array}{l}\text { Centris (Heterocentris) analis (Fabricius, 1804) } \\
\text { Centris (Centris) flavifrons (Fabricius, 1775) } \\
\text { Epicharis (Epicharana) flava Friese, } 1900\end{array}$ \\
\hline Emphorini & Diadasina sp. \\
\hline Ericrocidini & Acanthopus sp. \\
\hline Eucerini & Melissodes sp. \\
\hline Manueliini & Manuelia postica (Spinola, 1851) \\
\hline Tapinotaspidini & Tapinotaspoides sp. \\
\hline Tetrapediini & Tetrapedia maura Cresson, 1878 \\
\hline
\end{tabular}


Table 2. (next page) Matrix of character state distribution used in the cladistics analysis of the morphological dataset. Symbols: - = inapplicable; ? = missing/doubtful. Abbreviations: ANT, Anthophirini; API, Apina; BOM, Bombina; CEN, Centridini; EMP, Emphorini; ERI, Ericrocidini; EUC, Eucerini; EUG, Euglossina; MAN, Manueliini; MEL, Meliponina; TAP, Tapinotaspidini; TET, Tetrapediini. 


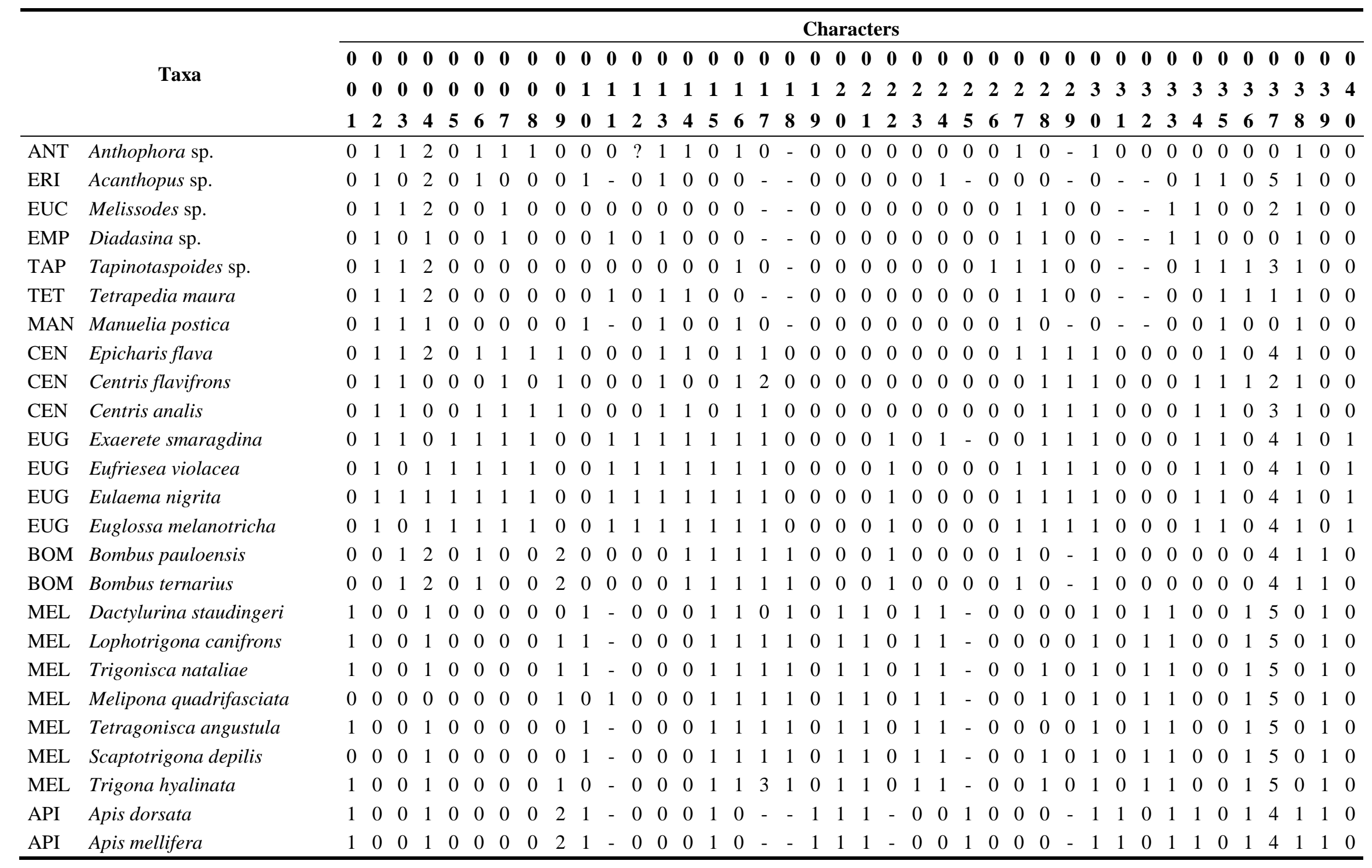


Table 2. Continuation.

\section{Characters}

Taxa

$\begin{array}{lllllllllllllllllllllllllllllllllllllllll}\mathbf{0} & \mathbf{0} & \mathbf{0} & \mathbf{0} & \mathbf{0} & \mathbf{0} & \mathbf{0} & \mathbf{0} & \mathbf{0} & \mathbf{0} & \mathbf{0} & \mathbf{0} & \mathbf{0} & \mathbf{0} & \mathbf{0} & \mathbf{0} & \mathbf{0} & \mathbf{0} & \mathbf{0} & \mathbf{0} & \mathbf{0} & \mathbf{0} & \mathbf{0} & \mathbf{0} & \mathbf{0} & \mathbf{0} & \mathbf{0} & \mathbf{0} & \mathbf{0} & \mathbf{0} & \mathbf{0} & \mathbf{0} & \mathbf{0} & \mathbf{0} & \mathbf{0} & \mathbf{0} & 0 & 0 & 0 & 0 & 0\end{array}$ $\begin{array}{lllllllllllllllllllllllllllllllllllllllll}4 & 4 & 4 & 4 & 4 & 4 & 4 & 4 & 4 & 5 & 5 & 5 & 5 & 5 & 5 & 5 & 5 & 5 & 5 & 6 & 6 & 6 & 6 & 6 & 6 & 6 & 6 & 6 & 6 & 7 & 7 & 7 & 7 & 7 & 7 & 7 & 7 & 7 & 7 & 8\end{array}$ $\begin{array}{lllllllllllllllllllllllllllllllllllllllll}1 & 2 & 3 & 4 & 5 & 6 & 7 & 8 & 9 & 0 & 1 & 2 & 3 & 4 & 5 & 6 & 7 & 8 & 9 & 0 & 1 & 2 & 3 & 4 & 5 & 6 & 7 & 8 & 9 & 0 & 1 & 2 & 3 & 4 & 5 & 6 & 7 & 8 & 9 & 0\end{array}$

\section{ANT Anthophora sp.}

ERI Acanthopus sp.

EUC Melissodes sp.

EMP Diadasina sp.

TAP Tapinotaspoides sp.

TET Tetrapedia maura

MAN Manuelia postica

CEN Epicharisflava

CEN Centris flavifrons

CEN Centris analis

EUG Exaerete smaragdina

EUG Eufriesea violacea

EUG Eulaema nigrita

EUG Euglossa melanotricha

BOM Bombus pauloensis

BOM Bombus ternarius

MEL Dactylurina staudingeri

MEL Lophotrigona canifrons

MEL Trigonisca nataliae

MEL Melipona quadrifasciata

MEL Tetragonisca angustula

MEL Scaptotrigona depilis

MEL Trigona hyalinata

API Apis dorsata

API Apis mellifera $\begin{array}{lllllllllllllllllllllllllllllllllllllllll}0 & 0 & 0 & 1 & 0 & 0 & 0 & - & - & 0 & 0 & 1 & 1 & 1 & 0 & 0 & 0 & 0 & 0 & 2 & 0 & 1 & 0 & 0 & 1 & 0 & 2 & 1 & 1 & 0 & 0 & 2 & 1 & 0 & 2 & 0 & 0 & - & - & 0\end{array}$

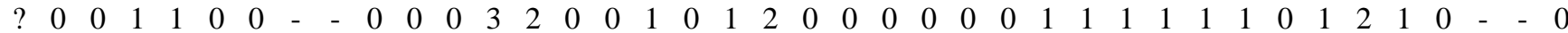

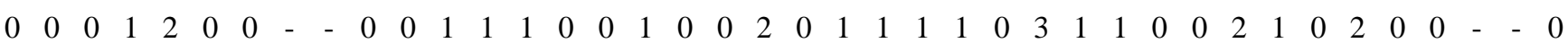

$\begin{array}{lllllllllllllllllllllllllllllllllllllllll}0 & 0 & 0 & 1 & 1 & 0 & 1 & 1 & 1 & 0 & 0 & 0 & 1 & 1 & 1 & 0 & 0 & 0 & 0 & 2 & 0 & 1 & 0 & 0 & 1 & 0 & 3 & 1 & 1 & 0 & 0 & 1 & 0 & 1 & 2 & 0 & 0 & - & - & 0\end{array}$

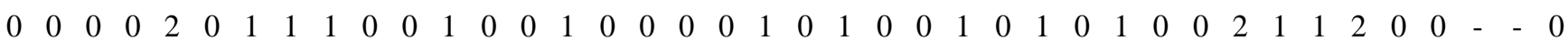

$\begin{array}{lllllllllllllllllllllllllllllllllllllllll}1 & 1 & 0 & 0 & 2 & 0 & 1 & 0 & 3 & 0 & 0 & 1 & 0 & 0 & 0 & 0 & 0 & 0 & 0 & 1 & 0 & 1 & 0 & 0 & 1 & 0 & 1 & 0 & 0 & 0 & 0 & 1 & 0 & 0 & 1 & 0 & 0 & - & - & 0\end{array}$

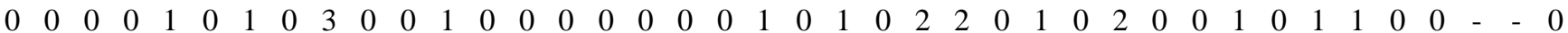

$\begin{array}{lllllllllllllllllllllllllllllllllllllllll}0 & 0 & 0 & 1 & 0 & 0 & 1 & 1 & 1 & 0 & 0 & 1 & 1 & 2 & 0 & 0 & 1 & 0 & 1 & 2 & 0 & 1 & 1 & 0 & 1 & 0 & 2 & 1 & 1 & 0 & 0 & 1 & 0 & 1 & 2 & 0 & 0 & - & - & 0\end{array}$

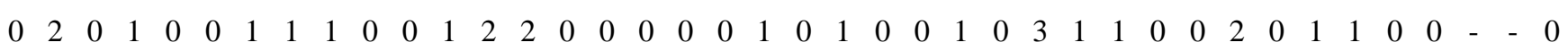

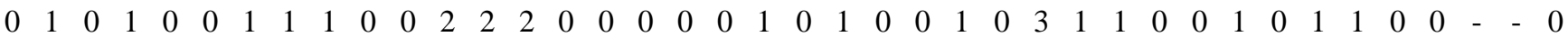
$\begin{array}{lllllllllllllllllllllllllllllllllllllllll}0 & 1 & 0 & 1 & 0 & 0 & 1 & 1 & 1 & 0 & 1 & 2 & 1 & 2 & 0 & 1 & 1 & 0 & 1 & 2 & 0 & 1 & 2 & 1 & 2 & 0 & 0 & 1 & 2 & 0 & 0 & 0 & 2 & 0 & 2 & 2 & 0 & - & - & 0\end{array}$ $\begin{array}{lllllllllllllllllllllllllllllllllllllllll}0 & 0 & 0 & 1 & 0 & 0 & 1 & 1 & 1 & 0 & 1 & 1 & 2 & 2 & 0 & 1 & 1 & 0 & 1 & 2 & 0 & 1 & 2 & 1 & 2 & 0 & 0 & 1 & 1 & 1 & 0 & 0 & 2 & 0 & 1 & 2 & 1 & 1 & 1 & 0\end{array}$ $\begin{array}{lllllllllllllllllllllllllllllllllllllllll}0 & 0 & 0 & 1 & 0 & 0 & 1 & 1 & 1 & 0 & 1 & 1 & 2 & 2 & 0 & 1 & 1 & 0 & 1 & 2 & 0 & 1 & 2 & 1 & 2 & 0 & 0 & 1 & 1 & 1 & 0 & 0 & 2 & 0 & 0 & 2 & 1 & 1 & 1 & 0\end{array}$

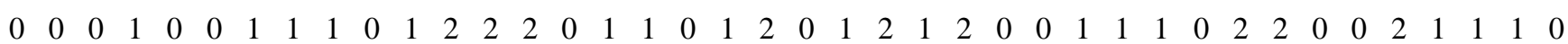
$\begin{array}{lllllllllllllllllllllllllllllllllllllllll}1 & 0 & 1 & 1 & 0 & 0 & 1 & 1 & 0 & 0 & 1 & 1 & 2 & 2 & 1 & 1 & 0 & 0 & 1 & 1 & 0 & 1 & 1 & 1 & 2 & 1 & - & 1 & 1 & 0 & 0 & 2 & 1 & 1 & 1 & 2 & 1 & 0 & 0 & 0\end{array}$ $\begin{array}{lllllllllllllllllllllllllllllllllllllllll}1 & 1 & 1 & 1 & 1 & 0 & 1 & 1 & 0 & 0 & 1 & 1 & 2 & 2 & 1 & 1 & 0 & 0 & 1 & 1 & 0 & 1 & 1 & 1 & 2 & 1 & - & 1 & 1 & 0 & 0 & 2 & 1 & 1 & 1 & 2 & 1 & 0 & 0 & 0\end{array}$ $\begin{array}{lllllllllllllllllllllllllllllllllllllllll}1 & 2 & 2 & 1 & 1 & 1 & 1 & 1 & 2 & 1 & 1 & 0 & 2 & 2 & 1 & 0 & 2 & 1 & - & 0 & 1 & 2 & 1 & 2 & 2 & 0 & 2 & 0 & 0 & 1 & 1 & 1 & 0 & 1 & 0 & 2 & 1 & 0 & 1 & 1\end{array}$ $\begin{array}{lllllllllllllllllllllllllllllllllllllllll}1 & 2 & 2 & 1 & 1 & 1 & 0 & - & - & 1 & 1 & 0 & 2 & 2 & 1 & 0 & 2 & 1 & - & 0 & 1 & 1 & 1 & 2 & 2 & 0 & 2 & 0 & 0 & 1 & 1 & 0 & 0 & 1 & 0 & 2 & 1 & 0 & 1 & 1\end{array}$ $\begin{array}{lllllllllllllllllllllllllllllllllllllllll}1 & 1 & 2 & 1 & 1 & 1 & 1 & 1 & 2 & 1 & 1 & 0 & 2 & 2 & 1 & 0 & 1 & 1 & - & - & - & - & - & - & - & 0 & 3 & 0 & 0 & 1 & 1 & 0 & 0 & 1 & 0 & 2 & 1 & 0 & 2 & 1\end{array}$

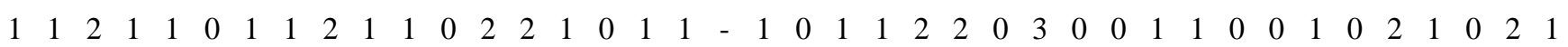

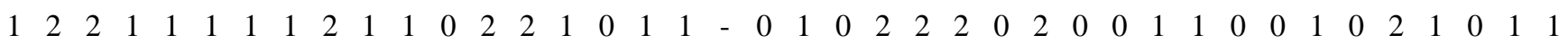
$\begin{array}{lllllllllllllllllllllllllllllllllllllllll}1 & 1 & 2 & 1 & 0 & 1 & 1 & 1 & 2 & 1 & 1 & 0 & 2 & 2 & 1 & 0 & 1 & 1 & - & 0 & 1 & 1 & 1 & 2 & 2 & 0 & 2 & 0 & 0 & 1 & 1 & 0 & 0 & 1 & 0 & 2 & 1 & 0 & 2 & 1\end{array}$

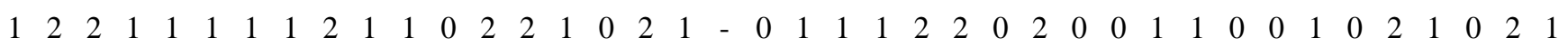
$\begin{array}{lllllllllllllllllllllllllllllllllllllllll}1 & 2 & 2 & 0 & 0 & 1 & 0 & - & - & 0 & 1 & 2 & 1 & 2 & 0 & 1 & 2 & 0 & 1 & 0 & 1 & 2 & 1 & 2 & 2 & 0 & 3 & 0 & 1 & 1 & 1 & 1 & 2 & 1 & 1 & 2 & 1 & 0 & 0 & 1\end{array}$ \begin{tabular}{lllllllllllllllllllllllllllllllllllllllll}
1 & 2 & 2 & 0 & 0 & 1 & 0 & - & - & 0 & 1 & 2 & 1 & 2 & 0 & 1 & 2 & 0 & 1 & 2 & 1 & 2 & 2 & 2 & 2 & 0 & 3 & 0 & 1 & 1 & 1 & 1 & 2 & 1 & 0 & 2 & 1 & 0 & 0 & 1 \\
\hline
\end{tabular} 
Table 2. Continuation.

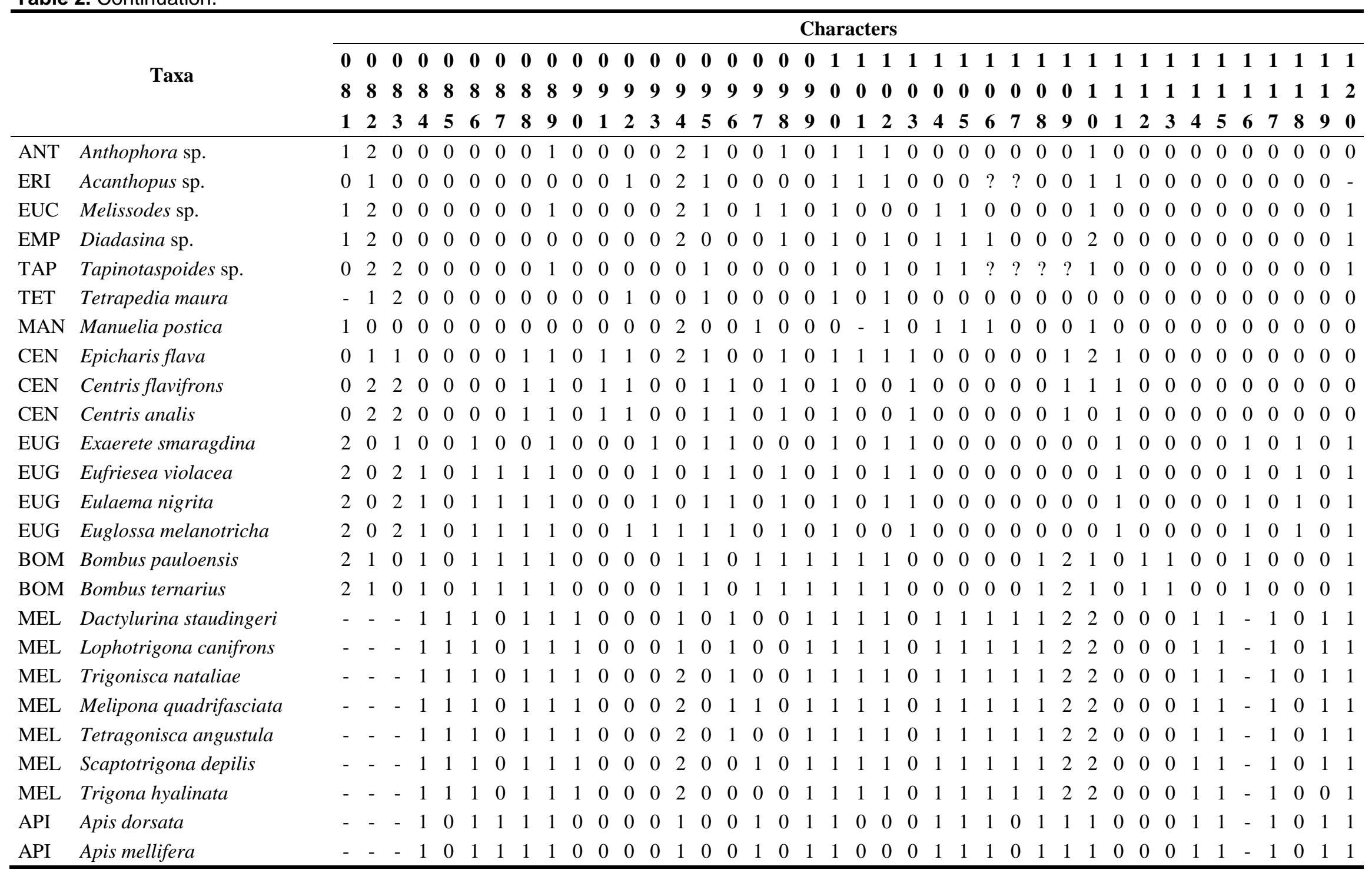


Table 2. Continuation.

\begin{tabular}{|c|c|c|c|c|c|c|c|c|c|c|c|c|c|c|c|c|c|}
\hline & \multirow{4}{*}{ Taxa } & \multicolumn{16}{|c|}{ Characters } \\
\hline & & 1 & & 1 & 1 & 1 & 1 & 1 & 1 & 1 & $\mathbf{1}$ & 1 & 1 & & & & \\
\hline & & 2 & 2 & 2 & 2 & 2 & 2 & 2 & 2 & 2 & 3 & 3 & 3 & 3 & 3 & 3 & \\
\hline & & 1 & 2 & 3 & 4 & 5 & 6 & 7 & 8 & 9 & $\mathbf{0}$ & 1 & 2 & 3 & 4 & & \\
\hline ANT & Anthophora sp. & - & 0 & 0 & 1 & 0 & 1 & 0 & 0 & 0 & 1 & 1 & 1 & 1 & 1 & c & ) \\
\hline ERI & Acanthopus sp. & 0 & 0 & 1 & 0 & 0 & 0 & 0 & 0 & 0 & 1 & 0 & 1 & 0 & 1 & c & ) \\
\hline EUC & Melissodes sp. & 0 & 0 & 0 & 1 & 0 & 1 & 1 & 0 & 0 & 0 & 0 & 0 & 0 & 1 & 2 & 2 \\
\hline EMP & Diadasina sp. & 0 & 0 & 0 & 1 & 0 & 1 & 1 & 1 & 0 & 0 & 0 & 0 & 0 & 1 & 2 & 2 \\
\hline TAP & Tapinotaspoides sp. & 0 & 0 & 0 & 1 & 0 & 1 & 1 & 1 & 1 & 0 & 0 & 0 & 0 & 1 & 2 & 2 \\
\hline TET & Tetrapedia maura & 0 & 0 & 1 & 1 & 0 & 1 & 1 & 0 & 1 & 0 & 0 & 0 & 0 & 1 & 2 & 2 \\
\hline MAN & Manuelia postica & - & 0 & 0 & 1 & 0 & 1 & 0 & 1 & 0 & 0 & 0 & 0 & 0 & 1 & c & 0 \\
\hline CEN & Epicharis flava & - & 0 & 0 & 0 & 0 & 1 & 1 & 1 & 0 & 1 & 1 & 1 & 0 & 1 & 1 & 1 \\
\hline CEN & Centris flavifrons & - & 0 & 0 & 0 & 0 & 1 & 1 & 1 & 0 & 1 & 1 & 1 & 1 & 1 & 1 & 1 \\
\hline CEN & Centris analis & - & 0 & 0 & 1 & 0 & 1 & 1 & 1 & 0 & 1 & 1 & 1 & 1 & 1 & 1 & 1 \\
\hline EUG & Exaerete smaragdina & 0 & 0 & 0 & 1 & 1 & 1 & 1 & 0 & 0 & 1 & 1 & 0 & 0 & 1 & 2 & 2 \\
\hline EUG & Eufriesea violacea & 1 & 1 & 0 & 1 & 1 & 1 & 1 & 1 & 0 & 1 & 1 & 0 & 0 & 1 & 2 & 2 \\
\hline EUG & Eulaema nigrita & 1 & 1 & 0 & 0 & 1 & 1 & 1 & 1 & 0 & 1 & 1 & 0 & 0 & 1 & 2 & 2 \\
\hline EUG & Euglossa melanotricha & 1 & 1 & 1 & 1 & 1 & - & 0 & 1 & 0 & 1 & 0 & 0 & 0 & 1 & 2 & 2 \\
\hline $\mathrm{BOM}$ & Bombus pauloensis & - & 0 & 0 & 0 & 0 & 1 & 1 & 0 & 0 & 2 & 0 & 0 & 1 & 1 & c & 0 \\
\hline $\mathrm{BOM}$ & Bombus ternarius & - & 0 & 0 & 0 & 0 & 1 & 1 & 0 & 0 & 2 & 0 & 0 & 1 & 1 & c & 0 \\
\hline MEL & Dactylurina staudingeri & 0 & 0 & 1 & 0 & 0 & 1 & 0 & 1 & 0 & 2 & 0 & 0 & 0 & 0 & c & 0 \\
\hline MEL & Lophotrigona canifrons & - & 0 & 1 & 0 & 0 & 0 & 0 & 0 & 0 & 2 & 0 & 0 & 0 & 0 & c & 0 \\
\hline MEL & Trigonisca nataliae & 0 & 0 & 1 & 0 & 0 & 0 & 0 & 1 & 0 & 2 & 0 & 0 & 0 & 0 & c & 0 \\
\hline MEL & Melipona quadrifasciata & 0 & 0 & 1 & 0 & 0 & 1 & 0 & 1 & 0 & 2 & 0 & 0 & 0 & 0 & c & 0 \\
\hline MEL & Tetragonisca angustula & - & 0 & 1 & 0 & 0 & 0 & 0 & 0 & 0 & 2 & 0 & 0 & 0 & 0 & c & 0 \\
\hline MEL & Scaptotrigona depilis & - & 0 & 1 & 0 & 0 & 0 & 0 & 0 & 0 & 2 & 0 & 0 & 0 & 0 & c & 0 \\
\hline MEL & Trigona hyalinata & - & 0 & 1 & 0 & 0 & 1 & 0 & 1 & 0 & 2 & 0 & 0 & 0 & 0 & c & 0 \\
\hline API & Apis dorsata & 0 & 0 & 1 & 1 & 0 & 0 & 0 & 0 & 0 & 2 & 0 & 0 & 1 & 1 & ( & 0 \\
\hline API & Apis mellifera & 0 & 0 & 1 & 1 & 0 & 0 & 0 & 0 & 0 & 2 & 0 & 0 & 1 & 1 & ( & 0 \\
\hline
\end{tabular}


Table 3. Nodal support values of Figure 3 estimated with standard bootstrapping, symmetrical resampling, and Bremer support calculations. Values for standard bootstrap and symmetrical resampling represent the absolute frequencies of recovering a given node with 10.000 replications.

\begin{tabular}{cccc}
\hline Nodes & $\begin{array}{c}\text { Standard } \\
\text { Bootstrap }\end{array}$ & $\begin{array}{c}\text { Symetrical } \\
\text { Resampling }\end{array}$ & Bremer \\
\hline & & & \\
$\mathbf{1}$ & 48 & 59 & 3 \\
$\mathbf{2}$ & 43 & 51 & 1 \\
$\mathbf{3}$ & 54 & 68 & 4 \\
$\mathbf{4}$ & 42 & 52 & 2 \\
$\mathbf{5}$ & 52 & 59 & 2 \\
$\mathbf{6}$ & 50 & 59 & 3 \\
$\mathbf{7}$ & 91 & 94 & 6 \\
$\mathbf{8}$ & 67 & 71 & 2 \\
$\mathbf{9}$ & 91 & 95 & 10 \\
$\mathbf{1 0}$ & 95 & 96 & 3 \\
$\mathbf{1 1}$ & 99 & 99 & 16 \\
$\mathbf{1 2}$ & 99 & 99 & 7 \\
$\mathbf{1 3}$ & 97 & 99 & 11 \\
$\mathbf{1 4}$ & 23 & 27 & 1 \\
& & & \\
\hline
\end{tabular}


Figure 1. (next page) Three sets of analyses conducted with the morphological dataset. A. Full dataset; to the left, strict consensus obtained from the four most parsimonious trees under a cladistics analysis with equal weights (EW); to the right, each one of the four most parsimonious trees showing the different positioning of Acanthopus (T1-T4); B. Reduced dataset, with Acanthopus excluded; to the left, strict consensus obtained from the two most parsimonious trees under a cladistics analysis with equal weights (EW); to the right, trees obtained from a cladistics analysis using implied weighting with different K values; C. Reduced dataset, with Acanthopus and continuous data excluded; to the left, strict consensus obtained from the two most parsimonious trees under a cladistics analysis with equal weights $(\mathrm{EW})$; to the right, trees obtained from a cladistics analysis using implied weighting with different $\mathrm{K}$ values. Star indicates the three used in the discussions (Figure 3). Abbreviations: A, Apina; B, Bombina; E, Euglossina; M, Meliponina. 

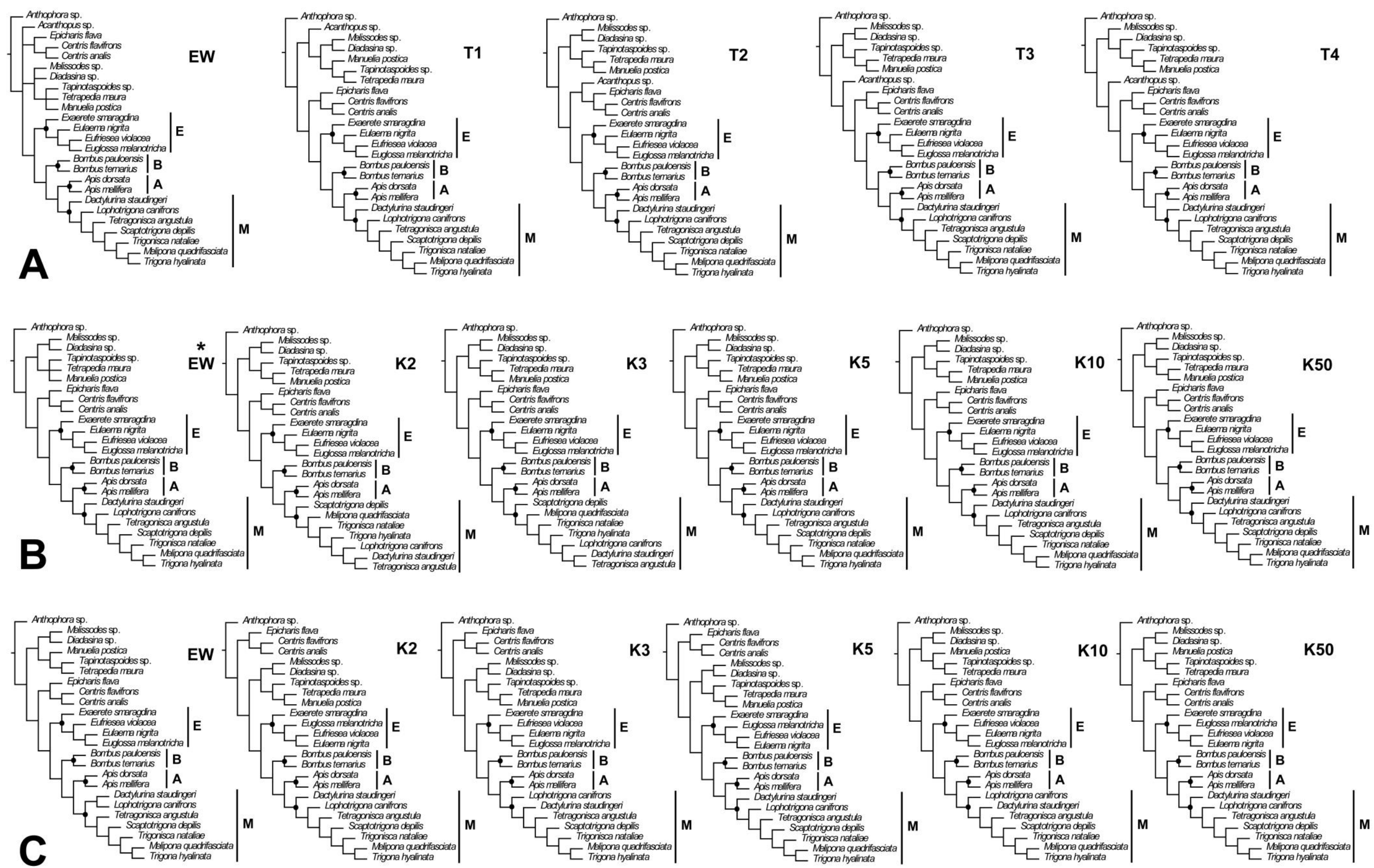


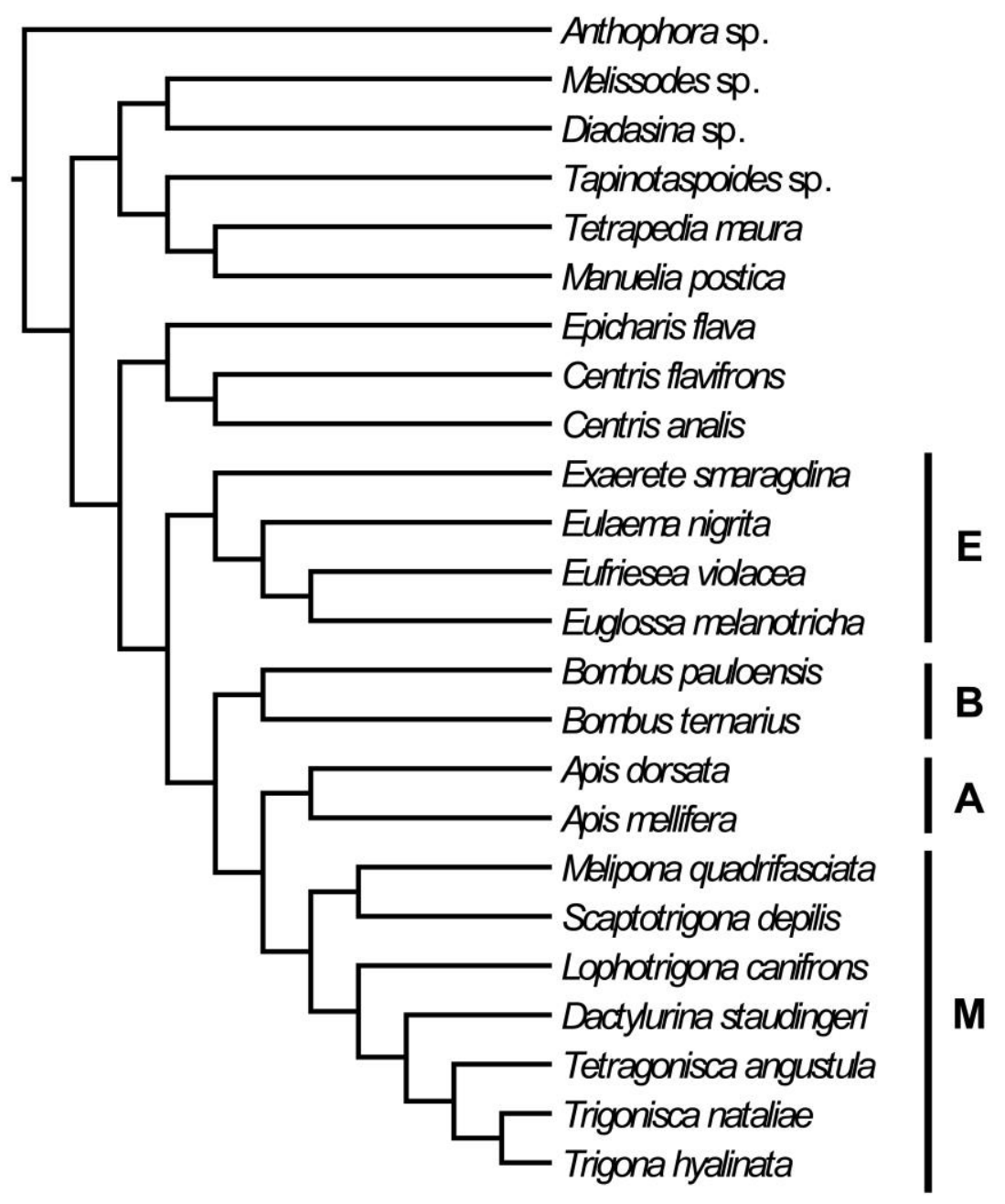

Figure 2. Tree resultant from the additional analysis $D$ with continuous data treated "as such". L: 389.214. Abbreviations: A, Apina; B, Bombina; E, Euglossina; M, Meliponina. 
Figure 3. (next page) Strict consensus obtained from the two most parsimonious trees resultant from analysis B. Open circles indicate homoplasious character-state changes; filled circles indicate unambiguous character-state changes; numbers above circles indicate the characters; number under the circles indicate the apomorphic character states; number inside boxes indicate the nodes referred in the discussion. L: $380, \mathrm{Cl}: 45, \mathrm{RI}: 78$. 


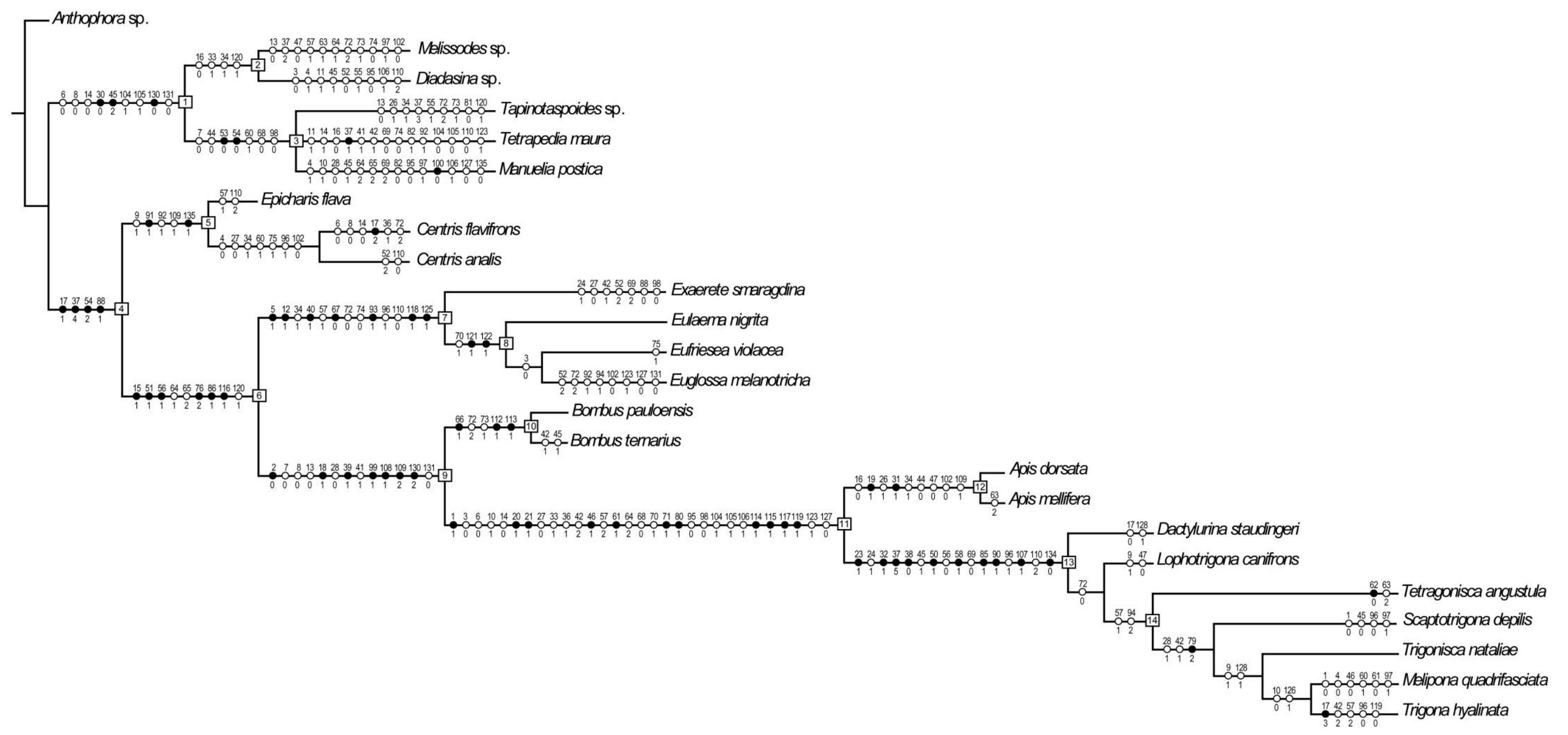


Figure 4. (next page) Optimization of transformations of the morphological characters constructed for this work onto phylogenetic hypotheses for corbiculate bees commonly recovered with morphological, behavioral and molecular data (see Cardinal \& Packer, 2007). A. Hypothesis commonly obtained with morphological/behavioral data. B. Hypothesis commonly obtained with the majority of molecular datasets. C. Alternative hypothesis recovered with some molecular datasets. Open circles indicate homoplasious character state changes; filled circles indicate unambiguous character state changes; numbers above circles indicate the characters; number under the circles indicate the apomorphic character states. Boxes on/above branches indicate ambiguous changes optimized with DELTRAN scheme. 


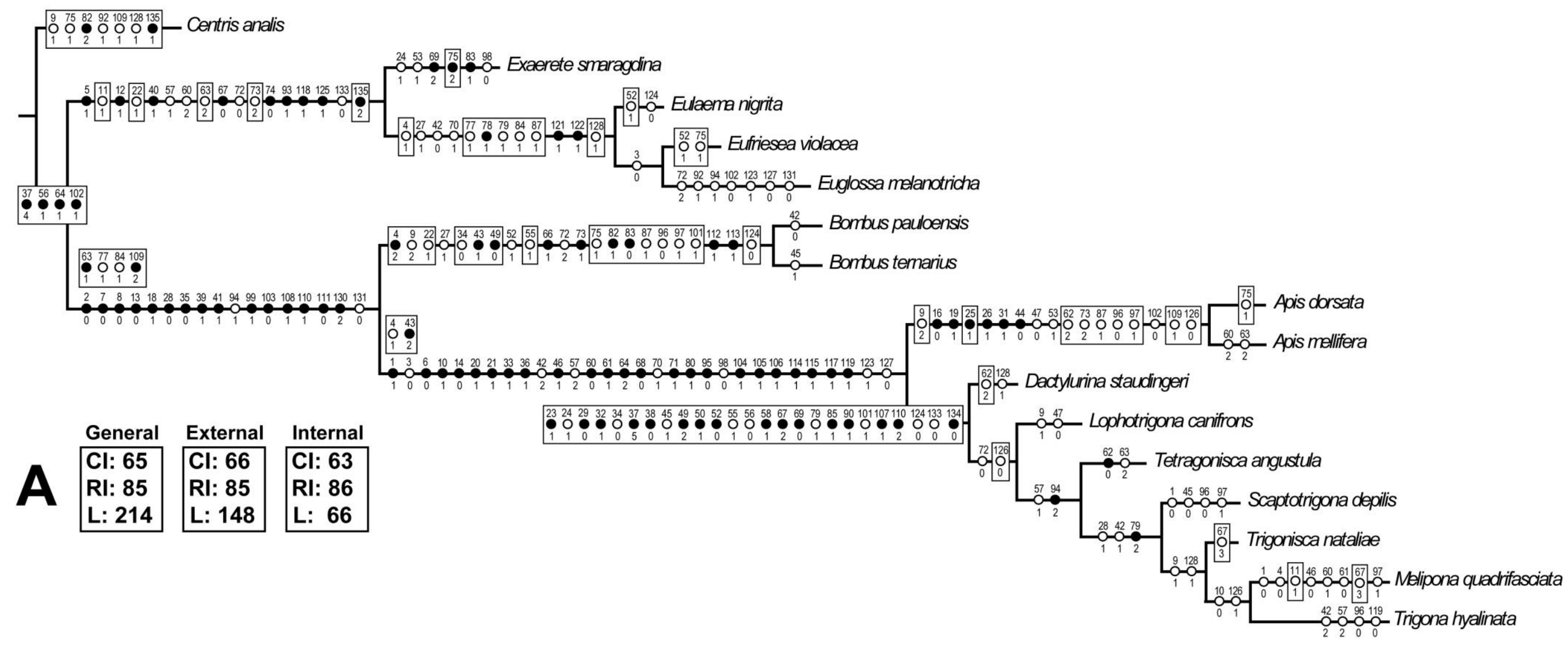




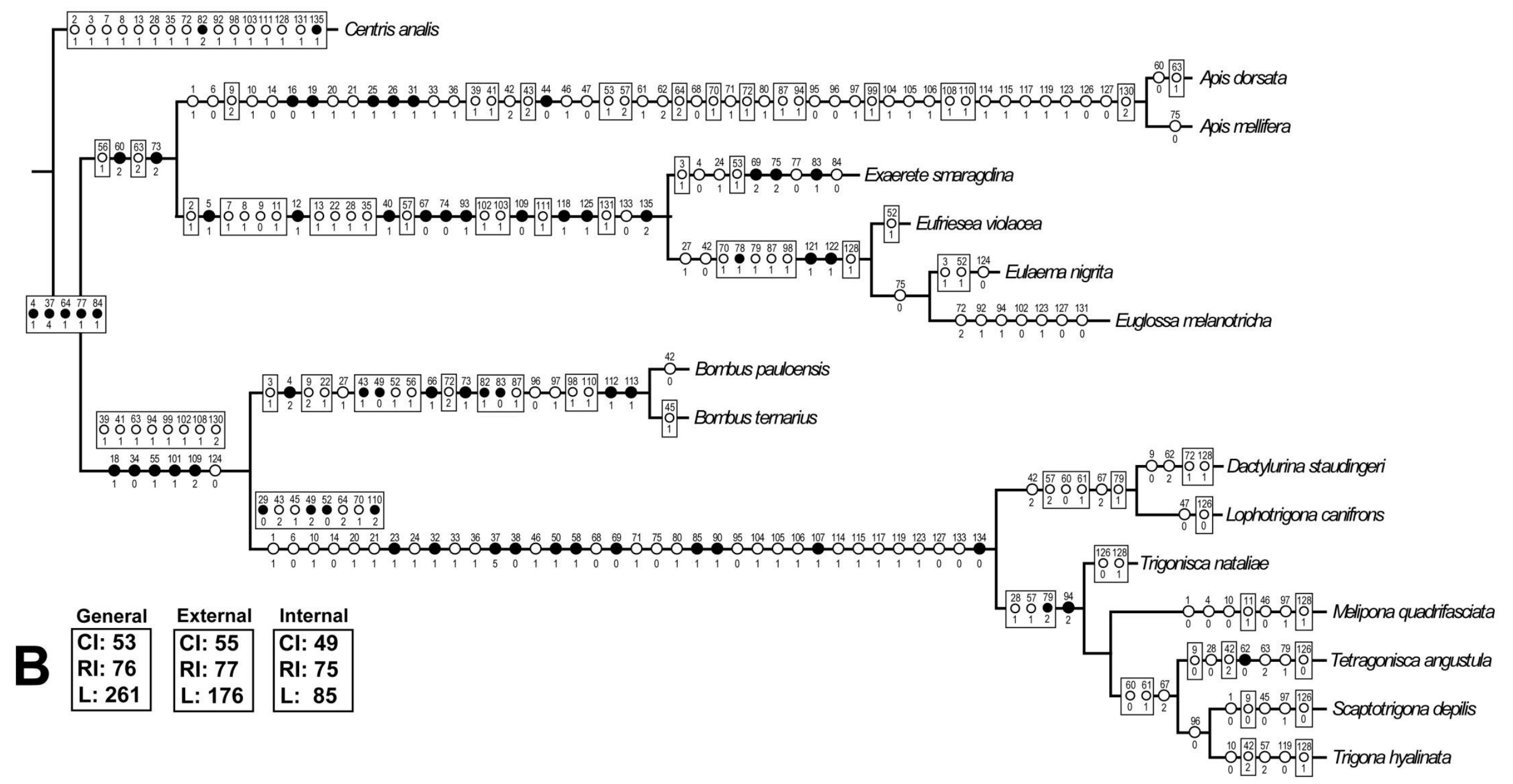

Figure 4. Continuation. 


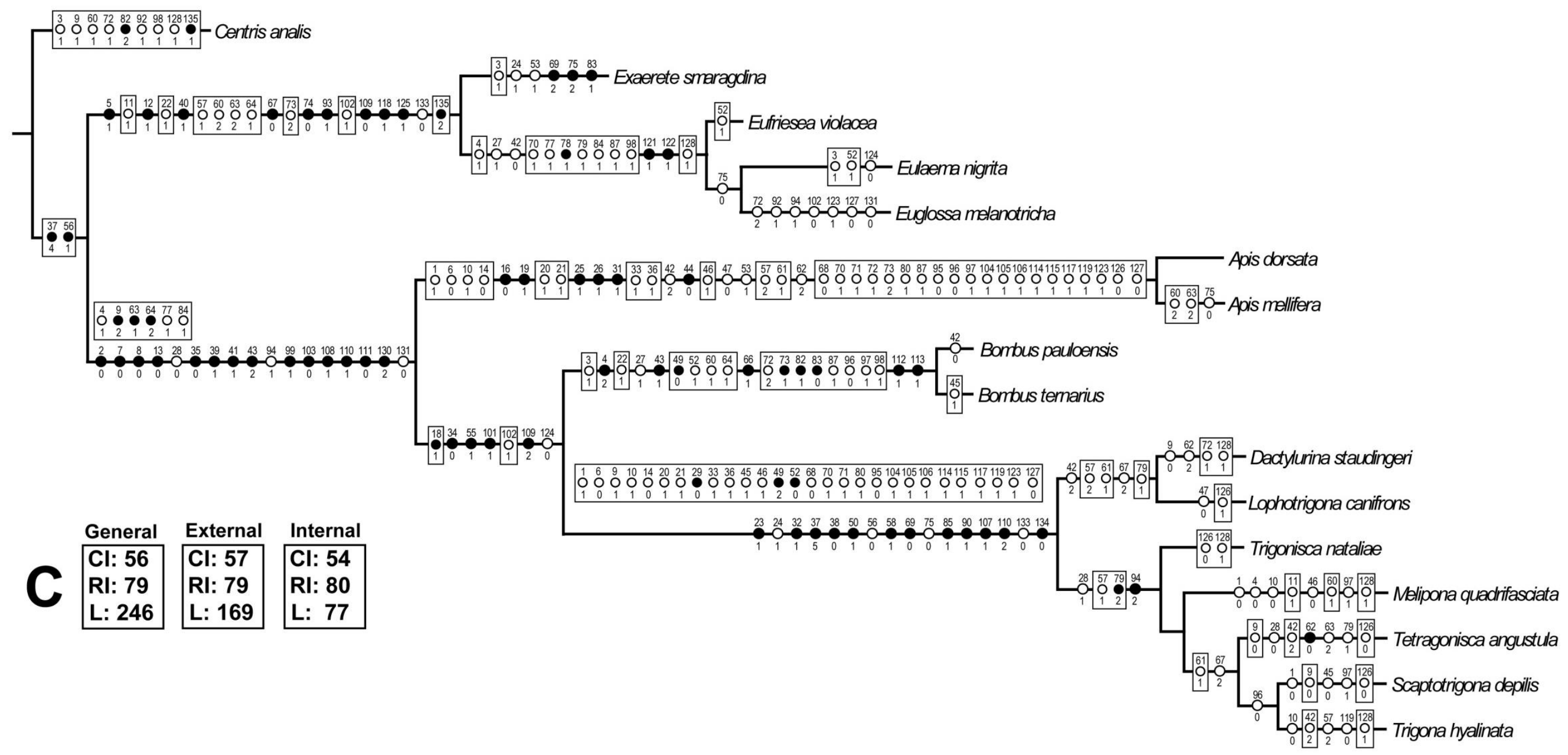

Figure 4. Continuation. 
Appendix 1. List of morphological characters in the data-matrix. The values of consistency indexes $(\mathrm{Ci})$ and retention indexes $(\mathrm{Ri})$ for each character were based on the tree obtained from analysis B. Characters encompassing continuous variation, but coded as discrete multistate, where indicated with asterisk $(*)$ in this list. Characters treated as discrete, but not included in analysis D were indicated with double asterisks (**).

\section{External skeletal morphology}

\section{Head}

1. Antennal foramina: (0) positioned approximately on the level of the median line of compound eyes; (1) positioned beneath the level of the median line of compound eyes. $\mathrm{Ci}$ : 33, Ri: 66.

Comments: Lowered positioned antennal foramina (state 1) are found only in Apina and most Meliponina.

2. (*) Distance between antennal foramina: (0) short; less than or equal to 1.5 times its diameter; (1) long; more than 2.5 times its diameter. Ci: 100, Ri: 100.

Comments: Closely approximate antennal foramina (state 0) are found in Bombina, Apina and Meliponina.

3. (*) Distance from inferior margin of antennal foramen to epistomal sulcus (modified from Melo, 1999: char. 18): (0) short; less than or equal to 0.5 times antennal foramen diameter; (1) long; between 0.5 and 1.0 times antennal foramen diameter. $\mathrm{Ci}$ : 33, Ri: 81.

Comments: Short distances (state 0) are found in Apina and Meliponina, although have also appeared probably convergently in a few other taxa.

4. (*) Anterior tentorial pit (modified from Roig-Alsina \& Michener, 1993: char. 2): (0) very low on epistomal sulcus; positioned more than 1.0 times antennal foramen diameter from the dorso-lateral angle of clypeus; (1) low on epistomal sulcus; positioned between 0.5 and 1.0 times antennal foramen diameter from the dorso-lateral angle of clypeus; (2) high on epistomal sulcus; positioned less than 0.5 times antennal foramen diameter from the dorso-lateral angle of clypeus. $\mathrm{Ci}: 28, \mathrm{Ri}: 44$.

Comments: Although this character has already been proposed by Roig-Alsina \& Michener (1993), they recognized only two states (i.e., high or low) to define the position of the 
anterior tentorial pit on epistomal sulcus. Here, three states are constructed (i.e., very low, low and high) trying to encompass all the variability found. High tentorial pits (state 2) are found in Bombina and most non-Corbiculate/Centridine taxa.

5. Epistomal sulcus on the lateral margin of ventral portion of clypeus (modified from Roig-Alsina \& Michener, 1993: char. 24): (0) slightly sinuous; (1) strongly sinuous; bent laterally at approximately 90 degrees. $\mathrm{Ci}: 100, \mathrm{Ri}: 100$.

Comments: The inferior portion of epistomal sulcus on the lateral margin of clypeus is strongly bent laterally (state 1) only in Euglossina, thus it seems to correspond to a synapomorphy for this clade.

6. Lateral margins of ventral portion of clypeus (modified from Roig-Alsina \& Michener, 1993: char. 6): (0) only slightly deflected posteriorly; angle of bent below 30 degrees; (1) strongly deflected posteriorly; angle of bent between 60 and 90 degrees. Ci: 33, Ri: 75 .

Comments: The character states proposed are best evaluated observing the head in ventral view. Strongly deflected lateral margins (state 1) are found in Bombina, Euglossina, most Centridini and a few other species. Although it can be probably correlated to the next character (char. 7), as most species which have strongly bent lateral margins also possess a conspicuously protruded clypeus, it was maintained as a separate character. It is important to consider that some of the above mentioned taxa (e.g., Bombus spp.) do not possess strongly protruding clypeus, whereas others (e.g., Diadasina sp. and Melissodes sp.) possess protruding clypeus even though they have only slightly bent lateral margins (state $0)$.

7. Clypeus: (0) not strongly protruded anteriorly; forming only slightly convex surface; (1) markedly protruded anteriorly; forming a distinct convex surface. Ci: 50, Ri: 88 .

Comments: The protrusion of clypeus was evaluated observing the head in profile. Strongly protruding clypeus (state 1) is defined when the distance between the insertion of mandible and the anterior limit of the convexity of clypeus is greater than 1.5 times antennal foramen diameter. State 1 is found in Euglossina, Centridini and a few other taxa, as Anthophora sp. 
8. Pleurostomal condyle (modified from Porto et al., 2015; CHAPTER 1 OF THIS THESIS): (0) contiguous with the lateral margin of ventral portion of clypeus; (1) partly covered by the lateral margin of ventral portion of clypeus. Ci: 33, Ri: 66 .

9. (*) Breadth of malar space: (0) short; less than or equal to 0.5 times antennal foramen diameter; (1) moderate; between 0.5 and 1.0 times antennal foramen diameter; (2) wide; more than 1.5 times antennal foramen diameter. $\mathrm{Ci}$ : 40, Ri: 66.

Comments: The breadth of malar space was measured from the ventral limit of the compound eye to the insertion of mandible. Conspicuously wide malar spaces (state 2) are found in Apina and Bombina.

10. Arched sulci of occiput (modified from Porto et al., 2015; CHAPTER 1 OF THIS THESIS): (0) present; (1) absent. Ci: 33, Ri: 71.

11. Ventral portion of arched sulci of occiput (modified from Porto et al., 2015; CHAPTER 1 OF THIS THESIS): (0) reaching the level of posterior tentorial pits; (1) ending before reach the level of posterior tentorial pits. Ci: 25, Ri: 50.

12. Surface of subforaminal bridge (modified from Porto et al., 2015; CHAPTER 1 OF THIS THESIS) (0) shallow or only moderately excavated; postoccipital pouch small; (1) very deep; postoccipital pouch large and distinct. Ci: 100, Ri: 100.

Comments: The condition of the subforaminal bridge presented by Anthophora sp. is rather complicated to interpret accurately. In this species, the surface of the bridge is very deep, with a large postoccipital pouch. However, the ventral portion of the pouch is broadly opened, being continuous with the hypostomal bridge. This condition is certainly not homologous to the one exhibited by all Euglossina (state 1). Thus, to avoid erroneous assumptions, Anthophora sp. is coded as “?” for this character.

\section{Mouthparts}

13. (*) Labrum (modified from Kimsey, 1984: char. 1; Roig-Alsina \& Michener, 1993: char. 7; Melo, 1999: char. 1): (0) rectangular; ratio of length to width less than or equal to 0.5 ; (1) quadrangular; ratio of length to width between 0.5 and 1.0. $\mathrm{Ci}: 33$, Ri: 80 . 
Comments: Roig-Alsina \& Michener (1993) have created only two states for this character - labrum broader than long and labrum longer than broad. All the taxa observed in this study have labra quadrangular (as broader as long) or rectangular (broader than long). Thus, only the variation in the ratios comprised in the range of less than 0.5 up to 1.0 was considered. More or less quadrangular (state 1) labrum is a common trait of Euglossina and almost all non-corbiculate taxa. Relatively short and distinctively rectangular labrum (state 0 ) is found in Bombina, Apina and Meliponina and a few other non-corbiculate taxa.

14. Rounded or oval spots of thin cuticle on the median proximal area of labrum (=labral bullae) (modified from Melo, 1999; char. 3): (0) absent; (1) present. Ci: 25, Ri: 66.

Comments: The labral bullae are present (state 1) in Bombina, Euglossina, most Centridini and a few other non-corbiculate Apidae. The condition exhibited in Bombina, however is slightly different from that present in Euglossina and most Centridini. In Bombina, the two spots are displaced laterally at the proximal lateral corners of labrum. If this displacement is just the result of the changes in shape that occurred in the labrum of the Bombina (i.e., rectangular labrum; char. 14:0) or if they are not homologous to the spots found in the other taxa is a matter of further discussions. Here, these spots are considered to be homologous, thus Bombina is coded as 1 for this character. In Tetrapedia maura, there is a third median spot between the other two. The former is considered to be a secondary acquisition, thus this taxa is coded 1 as well.

15. Mandibular apex (modified from Porto et al., 2015; CHAPTER 1 OF THIS THESIS): (0) conical; (1) spatulate. Ci: 100, Ri: 100.

Comments: State 0 is found in all non-corbiculate taxa, whereas state 1 is synapomorphic for Apini.

16. Preapical tooth (=tooth of pollex) (modified from Porto et al., 2015; CHAPTER 1 OF THIS THESIS): (0) absent; (1) present. Ci: 33, Ri: 50.

17. Number of pollex teeth: (0) one; (1) two; (2) three; (3) four. Ci: 75, Ri: 66.

Comments: The number of pollex teeth is relatively variable amongst the studied taxa. All the non-corbiculate/centridine taxa which possess a preapical tooth have only one tooth developed (state 0). In Apini and Centridini, most species have two developed teeth (state 
1). Three teeth (state 2) are found only in the pollex of $C$. flavifrons and four teeth (state 3) in Trigona hyalinata.

18. Pollex teeth (modified from Porto et al., 2015; CHAPTER 1 OF THIS THESIS): (0) well-developed, approximately as wide as the tooth of rutellum; (1) reduced, less than the half of the width of the tooth of rutellum. Ci: 100, Ri: 100 .

19. Acetabular groove of mandible (modified from Porto et al., 2015; CHAPTER 1 OF THIS THESIS): (0) present; (1) absent. Ci: 100, Ri: 100.

20. Condylar groove of mandible (modified from Porto et al., 2015; CHAPTER 1 OF THIS THESIS): (0) present; (1) absent. Ci: 100, Ri: 100.

21. Outer groove of mandible (modified from Porto et al., 2015; CHAPTER 1 OF THIS THESIS): (0) present; (1) absent. Ci: 100, Ri: 100.

22. Distal path of outer groove of mandible (modified from Porto et al., 2015; CHAPTER 1 OF THIS THESIS): (0) not subdivided; (1) subdivided into upper and lower ramus. Ci: 50, Ri: 80.

23. Oblique groove of mandibles (modified from Porto et al., 2015; CHAPTER 1 OF THIS THESIS): (0) absent; (1) present. Ci: 100, Ri: 100.

24. Comb of bristles in concavity on the distal posterior portion of stipes (modified from Roig-Alsina \& Michener, 1993: char. 29): (0) present; (1) absent. Ci: 50, Ri: 85.

Comments: This comb is present (state 0) in almost all taxa. The absence of the comb (state 1) occurs only in Meliponina and two cleptoparasitic taxa, Acanthopus sp. and Exaerete smaragdina.

25. Bristles of the comb in the stipital concavity: (0) strong; (1) thin. Ci: 100, Ri: 100. Comments: In almost all the taxa that possess a comb in the concavity on the distal posterior portion of maxillary stipes, the bristles are relatively strong (state 0 ) if compared to the other bristles on the surface of body. Only in Apina, the bristles of the comb are very 
thin (state 1). This reduction can represent an intermediate state between the conditions with strong bristles (state 0 ) and the one in which the comb is completely absent (e.g., Meliponina, char. 24: 1).

26. Stipital process of maxilla (=basistipital process of Winston, 1979; modified from Winston \& Michener, 1977: char. e; Kimsey, 1984: char. 3): (0) present; (1) absent. Ci: 50, Ri: 50.

Comments: Winston \& Michener (1977) have recognized the presence of this process (state 0) as a derived character state for the Meliponina. Kimsey (1984) has acknowledged that the presence of the process is plesiomorphic for Apini, as it was found also in Euglossina, Bombina and many other non-corbiculate taxa. The absence of the process (state 1) occurs only in Apina and convergently in Tapinotaspoides sp.

27. Proximal portion of maxillary stipes (modified from Prentice, 1991: Table 3.1, A, char. 6): (0) not projected over the base of cardo; (1) projected over the base of cardo. Ci: 33, Ri: 81.

Comments: State 1 is found in Bombina, non-cleptoparasitic Euglossina, and most noncorbiculate taxa. State 0 is found in Apina, Meliponina, Exarete smaragdina, Acanthopus sp. and Centris spp.

28. Longitudinal ridge on the external surface of maxillary stipes (modified from RoigAlsina \& Michener, 1993; char. 30): (0) absent; (1) present. Ci: 25, Ri: 62.

Comments: The development of the ridge and its position on the external surface of maxillary stipes are quite variable amongst different taxa. Probably, the ridges are not homologous throughout all the taxa, but to avoid unnecessary assumptions about the homology of this condition, the presence of an at least weak ridge was considered sufficient to the respective taxa in which this condition occurs be coded as 1 .

29. Longitudinal ridge on external surface of maxillary stipes: (0) not produced into a conspicuous lamella; (1) produced into a conspicuous marginal lamella. Ci: 50, Ri: 83.

Comments: In Euglossina and Centridini, the ridge on the external surface of maxillary stipes is produced into a thin lamella running along closely to the anterior margin of this sclerite. 
30. Subgaleal sclerite (=stipital sclerite of Winston, 1979; modified from Winston \& Michener, 1977: char. f; Roig-Alsina \& Michener, 1993; char. 40): (0) indistinct, completely fused to the remainder of stipes; (1) distinct, partially or not fused to the remainder of stipes. Ci: 100, Ri: 100 .

Comments: Distinct subgaleal sclerite (state 1) is found in Anthophora sp., Centridini and Apini.

31. Shape of subgaleal sclerite (=stipital sclerite of Winston, 1979): (0) thin; (1) distinctively broad. Ci: 100, Ri: 100.

Comments: State 1 is found only in Apina.

32. Distal portion of subgaleal sclerite (=stipital sclerite of Winston, 1979): (0) straight or rounded; (1) distinctively curved anteriorly, producing an "L-shaped" sclerite. Ci: 100, Ri: 100.

Comments: State 1 is found only in Meliponina.

33. Maxillary lacinia (modified from Roig-Alsina \& Michener, 1993; char. 38): (0) at least partially sclerotized; (1) weakly sclerotized or completely membranous. Ci: 50, Ri: 90.

Comments: Roig-Alsina \& Michener (1993) interpreted the laciniae of the species of Meliponina included in their work as being at least partly sclerotized. In this work, the laciniae of Meliponina is interpreted as being weakly sclerotized or completely membranous (state 1). The latter condition is found only in Apina, Meliponina, Melissodes sp. and Diadasina sp. However, the state of the laciniae in the latter two species is not exactly the same as in the former two. In Melissodes sp. and Diadasina sp. the lacinae are very weakly sclerotized but not completely membranous.

34. Internal surface of maxillary lacinia: (0) densely pubescent, at least partially covered with strong plumose bristles; (1) sparsely pubescent, most covered with thin simple bristles. Ci: 20, Ri: 60.

Comments: The states delimited for this character were evaluated only in $\mathrm{KOH}$ cleared specimens. Thus, some difficulties were found to decide the coding in some instances, especially in specimens whose bristles where very thin and/or translucent. The internal 
surface was considered densely pubescent (state 0) when at least its apical portion was covered with strong plumose hairs, otherwise it was coded as 1 . In some specimens, as most Euglossina and Centridini, strong bristles were present on the marginal area of the external surface of lacinia. In these taxa, however, the bristles were not observed on the internal surface, thus they were coded 1 as well. State 0 is found only in Bombina, Meliponina and a few other non-corbiculate taxa.

35. Posterior margin of galea (modified from Roig-Alsina \& Michener, 1993: char. 36): (0) fully sclerotized; (1) at least partly membranous. Ci: 33, Ri: 77.

Comments: In this work, an alternative view to that present by Roig-Alsina \& Michener (1993) is adopted. An at least partly membranous posterior margin of galeal blade is interpreted to occur in almost all studied taxa, thus state 1 is present in Euglossina and in the majority of non-corbiculate taxa.

36. (*) Ratio of the length of pre-palpal to post-palpal portions of maxillary galea: (0) less than or equal to $0.25 ;(1)$ more than 0.25 . Ci: 25 , Ri: 72.

Comments: The measures for the pre-palpal and post-palpal portions were taken from the base to the insertion of maxillary palpus and from it to the apex of the galeal blade, respectively. The maxillary palpus is considerably displaced from the base of galea (state 1) in Apina, Meliponina and a few other taxa.

37. Number of segments on maxillary palpus: (0) six; (1) five; (2) four; (3) three; (4) two; (5) one. Ci: 71, Ri: 80.

Comments: The number of palpal segments is much variable amongst the studied taxa. In general, a reduced number of segments - two (state 4) or one (state 5) - is found in Apini. The non-corbiculate taxa, on the other hand, usually preset a greater number of segments in the maxillary palpus, ranging from six to three.

38. (**) Segments of maxillary palpus: (0) more or less quadrangular; (1) elongate. Ci: 100, Ri: 100 .

Comments: The shape of each segment was evaluated through the ratio of is length to the width. More or less quadrangular segments have values ranging from 0.5 to 1.0. Elongate segments have values greater than 1.0, commonly between 1.5 and 2.0. State 0 is found 
only in Meliponina. All the other taxa have somewhat elongate segments on maxillary palpus (state 1).

39. Mentum and submentum (=lorum; modified from Plant \& Paulus, 1987; Roig-Alsina \& Michener, 1993: char. 43): (0) united; (1) separated. Ci: 100, Ri: 100.

Comments: Completely separated mentum and submentum (state 1) is found only in Bombina, Apina and Meliponina.

40. Basal margin of submentum (modified from Plant \& Paulus, 1987; Roig-Alsina \& Michener, 1993: char. 44): (0) entire; (1) with a deep indentation. Ci: 100, Ri: 100.

Comments: Roig-Alsina \& Michener (1993) have interpreted their representatives of Centridini and Anthophora paranensis Holmberg (1903) as exhibiting a deep indentation on the base of submentum. In this work, a deep cleft on the basal margin of the submentum is interpreted to be present only in Euglossina. Although the condition found in Anthophora sp. and the Centridini representatives in this work is similar, the base of submentum is only slightly emarginated, not deeply cleft as seen in Euglossina, thus they were also scored as 0 for this character.

41. (*) Ratio of length to the width of mentum: (0) more than 2.0; (1) less than or equal to 2.0. Ci: 50, Ri: 90.

Comments: The shape of the mentum was evaluated through the ratio of its length to width. In Euglossina and the majority of non-corbiculate taxa, the mentum is conspicuously elongate (state 0), with a somewhat rectangular shape. In Bombina, Apina and Meliponina, the mentum is shortened (state 1), with a more or less triangular shape. It is difficult to assess whether this shortening is directly correlated to the separation of the mentum and submentum (char. 39: 1) which occurs in these taxa or if they are independent conditions. As a relatively short mentum was also found in Tetrapedia maura whose mentum and submentum are not completely separated, the shortening of the submentum was interpreted as an independent condition from the union/separation of mentum and submentum. Also, the condition found in Acanthopus sp. is difficult to interpret accurately since the lorum of this species is composed only by a "V" shaped sclerite that probably correspond to the submentum. It seems that the mentum was much reduced (state 1?) or completely lost. Thus, this taxon was coded as "?" for this character. 
42. (*) Ratio of the length to the width of prementum (modified from Melo, 1999: char. 9): (0) more than or equal to 4.0; (1) between 4.0 and 3.0; (2) equal to or less than 3.0. Ci: 25 , Ri: 50.

Comments: The shape of the prementum is considerably variable amongst the observed taxa, and was roughly evaluated through the ration of its length to width. Relatively shortened prementa (state 2) are found in Apina and some Meliponina.

43. Subligular process of prementum (modified from Roig-Alsina \& Michener, 1993: char. 46): (0) completely sclerotized, fused to the remainder of the sclerite; (1) partially sclerotized, separated from the remainder of the sclerite by a weak sclerotic line; (2) weakly sclerotized, totally separated from the remainder sclerite. Ci: 100, Ri: 100.

Comments: State 1 is found only in Bombina and state 2 only in Apina and Meliponina.

44. First segment of labial palpus (modified from Roig-Alsina \& Michener, 1993: char. 50): (0) without an internal membranous margin; (1) with a distinct internal membranous margin. Ci: 50, Ri: 75.

Comments: State 0 is found only in Apina, Manuelia postica, Tetrapedia maura, and Tapinotaspoides sp.

45. (*) Ratio of the length of paraglossa to the length of first segment of labial palpus: (0) less than or equal to $0.5 ;(\mathbf{1})$ between 0.5 and $1.0 ;(2)$ equal to or more than $1.0 . \mathrm{Ci}: 33$, Ri: 60.

\section{Mesosoma}

46. Antero-lateral process of mesoscutum (modified from Prentice, 1991: Table 3.1, A, char. 7): (0) convex; (1) flat, projected posteriorly oven the supra-alar carina. Ci: 50, Ri: 85. Comments: State 1 is found in Apina and the majority of Meliponina.

47. Notauli: (0) absent; (1) present. Ci: 25, Ri: 25.

Comments: The notauli were considered absent when neither an internal ridge nor an external line of feebly sclerotized cuticle were distinguishable in cleared specimens, although in some instances it was difficult to accurately determine its presence or absence. 
48. Notauli: (0) weakly demarcated; indicated only externally by lines of less sclerotized cuticle on the surface of mesoscutum; (1) well-demarcated, indicated internally by heavy sclerotic longitudinal ridges. Ci: 50, Ri: 0.

Comments: State 0 is found only in Tetrapedia maura and Manuelia postica.

49. (**) Ratio of the length of the notaulus to the total length of mesoscutum: (0) less than or equal to $0.2 ;(\mathbf{1})$ between 0.2 and 0.33 ; (2) between 0.33 and $0.5 ;(3)$ equal to or more than 0.5. Ci: 75, Ri: 85.

Comments: The character states proposed here were meant to describe the extension of the notauli on the surface of mesoscutum. State 0 is found only in Bombina, where the notauli are restricted to the anterior-most portion of the mesoscutum. State 1 is found in Diadasina sp., Tapinotaspoides sp., Centridini and Euglossina. State 2 is found in most Meliponina. State 3 is found only in Tetrapedia maura and Manuelia postica.

50. Supra-alar carina of mesoscutum (modified from Melo, 1999: char. 54): (0) present; (1) present. Ci: 100, Ri: 100.

Comments: State 1 is found only in Meliponina.

51. Mesoscutellum (modified from Kimsey, 1984: char. 6): (0) not projected posteriorly; (1) projected posteriorly, extending at least partly above the metanotum. Ci: 100, Ri: 100. Comments: In all the corbiculate bees, the mesoscutellum is projected posteriorly, extending above the surface of the metanotum (state 1). This condition is most evident in the representatives of Euglossina and Bombina, but even in Apina and Meliponina, at least the median portion of the metanotum is partly covered by the mesoscutellum. Thus Apini were coded as 1 for this character. Although in Acanthopus sp. the mesoscutellum is also projected above the metanotum, this seems the consequence of the vertical configuration of the metanotum in profile (char. 53:3). Thus, this taxon where coded as 0 for this character.

52. (*) Ratio of the length, measured between the mesofurcal and metafurcal pits, to the width, measured between the mesocoxae, of the pseudosternal portions of mesepisterna/metepisterna: (0) less than or equal to 0.66; (1) between 0.67 and 1.5; (2) more than 1.5. Ci: 33, Ri: 63. 
Comments: This character was constructed to describe the variation found in the configuration of the pseudosternal portions of mesepisterna and metepisterna especially considering the positions of the furcal pits. In Acanthopus sp., Diadasina sp. and Meliponina, the mesofurcal and metafurcal pits are relatively approximate (state 0 ). In the remainder taxa, the configuration of this region of mesosoma is much more variable.

53. (**) Tilt angle of metanotum to the main longitudinal axis of the body (modified from Roig-Alsina \& Michener, 1993: char. 74): (0) low, less than or equal to 45 degrees; (1) moderate, between 45 and 75 degrees; (2) high, between 75 and 90 degrees; (3) very high, approximately 90 degrees. $\mathrm{Ci}: 40, \mathrm{Ri}: 62$.

54. (*) Ratio of the width, measured on the level of upper metapleural pit, to the high, measured from the apex of alar process to the ventral-most portion, of metepisternum (modified from Roig-Alsina \& Michener, 1993: char. 70): (0) less than or equal to 0.2; (1) between 0.2 and $0.3 ;(2)$ more than 0.3. Ci: 100, Ri: 100 .

Comments: State 0 is found only in Tapinotaspoides sp., Tetrapedia maura and Manuelia postica. State 1 is found only in Anthophora sp., Melissodes sp. and Diadasina sp. State 2 is found in Acanthopus sp., Centridini and Apini.

55. Conjunctive bonding the posterior portion of metasternum to the first metasomal sternum, between the metacoxae (modified from Roig-Alsina \& Michener, 1993: char. 72): (0) arising before the free distal margin of metasternum; (1) arising at the distal margin of metasternum. Ci: 25, Ri: 70.

Comments: State 1 is found only in Bombina, Meliponina, Tapinotaspoides sp. and Diadasina sp.

56. Area enclosed between the posterior portion of metasternum, metacoxae, and the first metasomal sternum (modified from Roig-Alsina \& Michener, 1993: char. 78): (0) entirely membranous; (1) with sclerotic bars close to the internal margins of metacoxae. $\mathrm{Ci}$ : 50 , Ri: 85.

Comments: State 1 is found only in Euglossina, Bombina and Apina. 
Wings

57. (*) Ratio of the length of marginal cell, measured on its posterior margin, to the length of $\mathrm{R}$ vein, measured from the base to the pterostigma (modified from Winston \& Michener, 1977: char. h): (0) less than or equal to 0.5; (1) between 0.5 and 0.66 ; (2) more than 0.66. Ci: 33, Ri: 66.

Comments: Winston \& Michener (1977) have recognized only the "unusual elongation of marginal and submarginal cells" (Winston \& Michener, 1977, p. 1136) in Apis spp. (state 2). In this work, the length of the marginal cell is further evaluated in relation to the length of the main sector of $\mathrm{R}$ vein, from its base to the pterostigma. Although the length of $\mathrm{R}$ vein can be possibly also varying, the ratio used here is meant to give a better evaluation of how the length of the marginal cell is varying amongst the observed taxa.

58. Marginal cell (modified from Roig-Alsina \& Michener, 1993: char. 103): (0) closed; (1) opened. Ci: 100, Ri: 100.

Comments: State 1 is found only in Meliponina.

59. Apex of marginal cell: (0) angulated; (1) rounded. Ci: 50, Ri: 85.

60. (*) Ratio of the length of the first recurrent vein $\left(1^{\text {st }} \mathrm{m}-\mathrm{cu}\right)$ to the length of $\mathrm{R}$ vein, measured from the base to the pterostigma: (0) less than or equal to $0.15 ;(\mathbf{1})$ between 0.16 and $0.2 ;(2)$ equal to or more than 0.2. Ci: 33, Ri: 66.

Comments: Trigonisca nataliae has much reduced posterior wing venation. This taxon was coded as inapplicable for this character.

61. First recurrent vein $\left(1^{\text {st }} \mathrm{m}-\mathrm{cu}\right)$ (modified from Michener, 1990: Table 1, char. 9): (0) obliquely oriented; (1) transversely oriented. Ci: 50, Ri: 83.

Comments: State 1 is found only in Apina and most Meliponina.

62. Path of first recurrent vein $\left(1^{\text {st }} \mathrm{m}-\mathrm{cu}\right)$ : (0) straight; (1) terminally angulated; (2) medially angulated. Ci: 66, Ri: 50 .

63. (*) Ratio of the length of the $M$ vein, measured from the base to the transversal vein $r$ $\mathrm{m}$, to the length of Rs vein, measured from the base to the transversal vein $r-m$, of the 
posterior wing: (0) more than or equal to 1.25 ; (1) between 1.25 and 0.75 ; (2) less than 0.75. Ci: 33, Ri: 63.

64. (*) Ratio of the length of second abscissa of $\mathrm{M}+\mathrm{Cu}$ vein, measured from the transversal vein cu-v to the bifurcation of $\mathrm{M}$ and $\mathrm{Cu}$, to the length of first abscissa of the same vein, measured from the base to the transversal vein cu-v, of the posterior wing: (0) less than or equal to 0.3 ; (1) between 0.3 and 0.6 ; (2) equal to or more than 0.6. Ci: 50, Ri: 83 .

Comments: State 1 is found in Melissodes sp., Euglossina and Bombina. State 2 is found in Manuelia postica, Apina and Meliponina. The remainder taxa present state 0.

65. (*) Ratio of the length of second abscissa of $\mathrm{M}+\mathrm{Cu}$ vein, measured from the transversal vein cu-v to the bifurcation of $\mathrm{M}$ and $\mathrm{Cu}$, to the length of the transversal vein cu-v of the posterior wing (modified from Roig-Alsina \& Michener, 1993: char. 106): (0) less than 1.0; (1) between 1.0 and 2.5; (2) more than 2.5. Ci: 50, Ri: 85.

Comments: State 0 is found only in Acanthopus sp. State 2 is found in Manuelia postica and Apini. The remainder taxa present state 1.

66. Jugal lobe of posterior wing (modified from Winston \& Michener, 1977: char. k; Michener, 1990: Table 1, char. 10): (0) present; (1) absent. Ci: 100, Ri: 100.

Comments: State 1 is found only in Bombina. Although the jugal lobe in Euglossina is much reduced, in this work the interpretation of Plant \& Paulus (1987) is adopted. These authors regard the patch of bristles present on the region where the jugal lobe should be present as its remnant. Thus, Euglossina was coded as 0 for this character.

67. (*) Ratio of the length of jugal lobe to the length of vanal lobe, both measured from the base of wing to the respective apices (modified from Roig-Alsina \& Michener, 1993: char. 105): (0) less than or equal to 0.15 ; (1) between 0.15 and 0.25 ; (2) between 0.25 and 0.5 ; (3) more than 0.5. Ci: 42, Ri: 63.

68. Papillation on the distal portion of wings (modified from Winston \& Michener, 1977: char. j): (0) inconspicuous; (1) well-developed. Ci: 50, Ri: 90. 
Legs

69. Malus of protibial spur: (0) slender; (1) robust; (2) very robust. Ci: 50, Ri: 75.

Comments: State 0 is found in Tetrapedia maura and Meliponina. State 2 is found only in Manuelia postica and Exaerete smaragdina. The remainder taxa present state 1.

70. Spines on the base of ventral margin of malus: (0) present; (1) absent. Ci: 50, Ri: 90.

Comments: State 1 is found in Acanthopus sp., non-cleptoparasitic Euglossina, Apina and Meliponina.

71. Spines on the dorsal margin of the trunk of protibial spur: (0) present; (1) absent. Ci: 100, Ri: 100.

Comments: State 1 is found in Acanthopus sp., Apina and Meliponina.

72. (*) Ratio of the length of malus to the length of velum: (0) less than 0.25 ; (1) between 0.25 and $0.5 ;(2)$ more than 0.5 . Ci: 25, Ri: 53.

73. Carina on the anterior margin of velum (=anterior velum of Schönitzer, 1985; modified from Kimsey, 1984: char. 10; Michener, 1990: Table 1, char. 6; Roig-Alsina \& Michener, 1993): (0) reduced or absent; (1) moderately developed; (2) strongly developed, produced into an apical spine. $\mathrm{Ci}: 33, \mathrm{Ri}: 55$.

Comments: State 2 is found only in Euglossina and Apina.

74. Distal angle of velum: (0) acute, normally projected; (1) blunt or rounded, not projected. Ci: 25, Ri: 50.

75. (*) Ratio of the length of mesotibial spur to the length of mesotibia: (0) less than or equal to $0.25 ;(\mathbf{1})$ between 0.25 and $0.35 ;(2)$ more than 0.35 . Ci: $25, \mathrm{Ri}: 50$.

76. Basitibial plate (modified from Roig-Alsina \& Michener, 1993: char. 84): (0) present; (1) much reduced; (2) absent. Ci: 100, Ri: 100.

Comments: State 1 is found only in Acanthopus sp. whereas state 2 is found in Apini. The true loss of the basitibial plate occurs only in the corbiculate bees. To distinguish this 
condition from the extreme reduction that occurs convergently in many cleptoparasitic taxa, state 1 was created to encompass the latter condition.

77. Glabrous and concave surface surrounded by long bristles on the external surface of metatibia (i.e., corbicula) (modified from Winston \& Michener, 1977: char. a): (0) absent; (1) present. Ci: 50, Ri: 88.

Comments: State 1 is found only in Apini (i.e., corbiculate bees) being reversed to state 0 in the cleptoparasitic euglossine Exaerete smaragdina.

78. Posterior angle of corbicula: (0) not projected or only slightly projected beyond the distal margin of metatibia; (1) conspicuously projected beyond the distal margin of metatibia. Ci: 100, Ri: 100.

Comments: State 1 is found only in the non-cleptoparasitic Euglossina.

79. Posterior angle of corbicula: (0) blunt; (1) rounded; (2) acute. Ci: 66, Ri: 83.

80. Metatibial spurs (modified from Winston \& Michener, 1977: char. q): (0) present; (1) absent. Ci: 100, Ri: 100.

Comments: State 1 is found only in Apina and Meliponina.

81. Bases of metatibial spurs (modified from Roig-Alsina \& Michener, 1993: char. 81): (0) not separated by sclerotic bridge; (1) partially separated by sclerotic bridge; (2) completely separated by sclerotic bridge. Ci: 66, Ri: 83 .

Comments: The interpretation of the character states given in this work is considerably different from that in Roig-Alsina \& Michener (1993). The bases of the metatibial spurs were considered not separated by a bridge when the distance between them was less than half its diameter and there was no indication of a sclerotic projection. State 1 was considered when the bases were distant at least half its diameter from each other and there was an incomplete sclerotic projection between them. State 2 was considered when the distance was equal of greater than half the diameter of the bases and a conspicuous sclerotic bridge was discernible between them. 
82. (*) Ratio of the length of the inner metatibial spur to the length of the metatibia: (0) less than or equal to 0.25 ; (1) between 0.25 and $0.35 ;(2)$ more than 0.35 . Ci: $40, \mathrm{Ri}: 57$.

83. Inner margin of the inner metatibial spur (modified from Roig-Alsina \& Michener, 1993: char. 82): (0) finely serrate; (1) roughly serrate; (2) pectinate. Ci: 33, Ri: 33.

Comments: State 1 is found only in Epicharis flava and Exaerete smaragdina. State 2 is found in the majority of Centridini and Euglossina, and in Tetrapedia maura and Tapinotaspoides sp.

84. Comb of strong bristles on the distal margin of metatibia (i.e., rastellum) (modified from Winston \& Michener, 1977: char. b): (0) absent; (1) present. Ci: 50, Ri: 88.

Comments: State 1 is found only in Apini being reversed to state 0 in the cleptoparasitic euglossine Exaerete smaragdina.

85. Tuff of long curved bristles on the anterior angle of the distal margin of metatibia (i.e., penicillum) (modified from Winston \& Michener, 1977: char. c): (0) absent; (1) present. Ci: 100, Ri: 100.

Comments: State 1 is found only in Meliponina.

86. Proximal articulation of posterior basitarsus (modified from Roig-Alsina \& Michener, 1993: char. 87): (0) close to the dorsal margin of metatibia; (1) displaced, far from the dorsal margin of metatibia. Ci: 100, Ri: 100.

Comments: State 1 is found only in Apini.

87. Posterior expansion of the basal portion of posterior basitarsus (i.e., auricle) (modified from Winston \& Michener, 1977: char. d): (0) absent; (1) present. Ci: 33, Ri: 66.

Comments: State 1 is found in Euglossina, with exception of Exaerete smaragdina, Bombina and Apina.

88. (*) Ratio of the length to the width of the posterior basitarsus (modified from RoigAlsina \& Michener, 1993; char. 90): (0) more than or equal to 3.0; (1) between 3.0 and 2.0. Ci: 50, Ri: 83 . 
Comments: State 1 is found in Centridini and Apini, with exception of Exaerete smaragdina.

89. Posterior basistarsus: (0) more or less cylindrical; (1) laterally compressed. Ci: 33, Ri: 0 .

Comments: Laterally compressed posterior basitarsi (state 1) is a common feature of almost all observed taxa. State 0 is found only in Acanthopus sp., Diadasina sp., Tetrapedia maura and Manuelia postica.

90. Apices of tarsal claws (modified from Roig-Alsina \& Michener, 1993: char. 99): (0) bifid, with inner tooth distinctively smaller than the outer one; (1) simple. Ci: 100, Ri: 100. Comments: Simple claws were observed in all the Meliponina representatives dissected for this study, although Roig-Alsina \& Michener (1993) have observed bifid claws (state 0) in Melipona fulva Lepeletier, 1836.

91. Tarsal claws: (0) bifid or simple on all legs; (1) bifid on the anterior and median pairs of legs, simple on the posterior pair of legs. Ci: 100, Ri: 100.

Comments: State 1 is found only in Centridini.

92. Arolia (modified from Kimsey, 1984: char. 15; Michener, 1990: Table 1, char. 5): (0) absent; (1) present. Ci: 33, Ri: 50.

Comments: Kimsey (1984) and Michener (1990) have regarded the arolia in Bombus spp. as being much reduced. This view is adopted here, since some of the elements that constitute the arolium are yet distinguishable. Thus, this taxon is coded as 1 for this character.

\section{Internal skeletal morphology}

Head

93. Lateral spur projecting from the dorsal sheet of anterior tentorial arm reaching to compound eye margin at level of antennal foramen (modified from Porto et al., 2015; CHAPTER 1 OF THIS THESIS): (0) absent; (1) present. Ci: 100, Ri: 100.

Comments: State 1 is found only in Euglossina. 
94. Attachment of the thickening of secondary tentorial bridge to the internal surface of head capsule (modified from Porto et al., 2015; CHAPTER 1 OF THIS THESIS): (0) not reaching the inferior margin of antennal foramen, delimiting a broad subantennal area; (1) nearly reaching the inferior margin of antennal foramen, delimiting a small triangular area; (2) reaching the inferior margin of antennal foramen, thus there is actually no space between them. Ci: 28, Ri: 58.

95. Lateral expansion of the attachment of the thickening of secondary tentorial bridge above epistomal ridge (modified from Porto et al., 2015; CHAPTER 1 OF THIS THESIS): (0) less than 0.5 antennal foramen diameter; (1) more than or equal to 0.5 antennal foramen diameter. Ci: 33, Ri: 80.

Comments: State 1 is found in Euglossina, Bombina, and the majority of non-corbiculate taxa.

96. Median expansion of epistomal ridge adjacent to the insertion of eutentorial arm to head capsule (modified from Porto et al., 2015; CHAPTER 1 OF THIS THESIS): (0) short; length less than 1.0 times antennal foramen diameter; (1) long; length more than 1.5 times antennal foramen diameter. Ci: 20, Ri: 60.

Comments: State 1 is found in Centridini, Euglossina and most Meliponina.

97. (**) Ventral ridge of clypeus (modified from Porto et al., 2015; CHAPTER 1 OF THIS THESIS): (0) broad, at least 0.33 times antennal foramen diameter; (1) reduced to a small strip, less than 0.33 times antennal foramen diameter. Ci: 16, Ri: 28.

98. Fan-shaped sheets of posterior tentorial arms (modified from Porto et al., 2015; CHAPTER 1 OF THIS THESIS.) (0) reduced; not delimiting broad cavities opened ventrally; (1) well-developed; delimiting broad cavities opened ventrally. Ci: 33, Ri: 80.

99. Tentorial bridge seen through occipital foramen (modified from Porto et al., 2015; CHAPTER 1 OF THIS THESIS): (0) straight; (1) arched ventrally. Ci: 100, Ri: 100. Comments: State 1 is found only in Bombina, Apina and Meliponina. 
100. Hypostoma (modified from Roig-Alsina \& Michener, 1993: char. 23): (0) not directly connected to the tentoria, fused to the secondary tentorial bridge only by a vertical sclerotic septum; (1) completely fused to the tentoria. Ci: 100, Ri: 0

Comments: Roig-Alsina \& Michener (1993) have made a distinction between the conditions were the hypostoma and tentoria are connected by a thicker septum (their state 1) and when they are completely connected (their state 2). In this work, only two states were recognized: fusion through a vertical septum (state 0 ) and complete fusion (state 1). In their work, Centris tricolor Friese, 1899 and Eufriesea violacea were coded as 1. Here, Centris analis, Centris flavifrons and Eufriesea violacea were interpreted as having completely fused hypostoma and tentoria (state 1). The "thicker septum" is interpreted here as a consequence of the fusion zone restricted to the median portion of the hypostomal bridge (char. 103:0).

101. Fusion of hypostoma to the secondary tentorial bridge (modified from Porto et al., 2015; CHAPTER 1 OF THIS THESIS) (0) restricted to the median area of hypostomal bridge; (1) forming a wide median sheet distinguishable by two lateral fusion lines. Ci: 25 , Ri: 70.

Comments: State 1 is found in Bombina, Meliponina and a few other non-corbiculate taxa.

102. Connection of hypostoma to tentoria (modified from Porto et al., 2015; CHAPTER 1 OF THIS THESIS): (0) restricted to the posterior forth of tentorial length or less; (1) extending for more than the posterior forth of tentorial length. Ci: 25, Ri: 40.

Comments: State 0 is found only in Apina, Euglossa melanotricha, Centris spp. and Melissodes sp.

103. Maxillary process of hypostoma (modified from Porto et al., 2015; CHAPTER 1 OF THIS THESIS): (0) located medially on hypostoma; (1) displaced anteriorly on hypostoma, nearly abutting against clypeus. Ci: 50, Ri: 83.

Comments: The anterior displacement of the maxillary processes of hypostoma (state 1) is found in Euglossina and Centridini. The conditions exhibited by these taxa, however, can have been influenced by the great development of the processes as a consequence to the increased size of most specimens. In Euglossina, it can be also related to the development 
of a long proboscis and the mechanical constraints associated to the reception of its base inside the proboscidial fossa.

104. Paramandibular process of hypostoma (modified from Porto et al., 2015; CHAPTER 1 OF THIS THESIS): (0) anteriorly produced; almost abutting against the internal surface of clypeus; (1) not produced; ending far away from the internal surface of clypeus. Ci: 33, Ri: 80.

Comments: State 1 is found only in Apina, Meliponina and a few other non-corbiculate taxa.

105. Paramandibular carina (modified from Porto et al., 2015; CHAPTER 1 OF THIS THESIS): (0) present; (1) absent. Ci: 33, Ri: 80.

Comments: In Euglossina, the carina is well-developed (state 0), but displaced to the anterior margin of the paramandibular process. In all the remainder taxa, which exhibit a well-developed carina, it is located on the dorsal margin of the process. It is notoriously that this carina is always present when the paramandibular process is conspicuously produced. Michener (1944) has already noted that the development of the carina seems to be related to that of the process, strengthening the whole structure in the taxa they co-occur. As it was observed in this study, despite the carina is always present when the process is produced, it exhibits a considerable degree of development, being nearly absent in some taxa as Anthophora sp. and Tetrapedia maura, even though the process is well-developed. Thus, these conditions were interpreted as independent character states.

106. Attachment point of frontal muscles on pharyngeal rods (modified from Porto et al., 2015; CHAPTER 1 OF THIS THESIS): (0) distinctly produced; (1) relatively small. Ci: 33, Ri: 80.

Comments: State 1 is found only in Apina, Meliponina and a few other non-corbiculate taxa.

107. Position of the attachment point of frontal muscles on pharyngeal rod (modified from Porto et al., 2015; CHAPTER 1 OF THIS THESIS): (0) at less than a fourth of the rod total length from its apex; (1) at more than a fourth of the rod total length from its apex. Ci: 100 , Ri: 100 . 
Comments: State 1 is found only in Meliponina.

108. (**) Hypopharyngeal lobe (modified from Porto et al., 2015; CHAPTER 1 OF THIS THESIS): (0) elongate; at least 0.75 times the length of the pharyngeal rods; (1) short; equal to or less than 0.5 times the length of the pharyngeal rods. Ci: 100, Ri: 100 .

Comments: State 1 is found only in Apina, Bombina and Meliponina.

109. Apex of hypopharyngeal lobe (modified from Porto et al., 2015; CHAPTER 1 OF THIS THESIS): (0) unilobate; (1) bilobate; (2) trilobate. Ci: 66, Ri: 91.

Comments: State 2 is found only in Bombina and Meliponina. State 1 is found only in Apina and Centridini. However, the condition presented by Apina, is certainly distinct from that exhibited by Centridini, as in the former the whole hypopharyngeal lobe has a more or less triangular shape with a deep arched emargination on its apex between the two lobes. Furthermore, a "trilobate" condition seems to be present in all taxa, except Apina, but in the taxa coded as "unilobate" the median lobe is much enlarged and overhangs the other two, giving the impression of a unilobate apex. This seems to be the true condition for Centridini, since in the cleared specimens an indication of a third median lobe was somewhat visible but the sitophore was partly damaged during the dissections. Thus, more specimens should be evaluated to access the best coding scheme for this character. For the time being, the coding scheme presented here will be maintained.

\section{Mesosoma}

110. (*) Tilt angle of the propleural arm (modified from Porto et al., in prep.; CHAPTER 2 OF THIS THESIS): (0) low; (1) moderate; (2) high. Ci: 33, Ri: 69.

111. (**) Sheet of the propleural arm (modified from Porto et al., in prep.; CHAPTER 2 OF THIS THESIS): (0) slender; (1) broad. Ci: 50, Ri: 83.

112. Anterior margin of the ventral propleural area (modified from Porto et al., in prep.; CHAPTER 2 OF THIS THESIS): (0) straight or only slightly emarginated; (1) strongly emarginated. Ci: 100, Ri: 100.

Comments: State 1 is observed only in Bombina. 
113. Anterior process of the prosternum (modified from Porto et al., in prep.; CHAPTER 2 OF THIS THESIS): (0) not expanded; (1) expanded laterally. Ci: 100, Ri: 100.

Comments: State 1 is found only in Bombina.

114. Lateral processes of the prosternum (modified from Porto et al., in prep.; CHAPTER 2 OF THIS THESIS): (0) weakly produced or nearly absent; (1) strongly produced. Ci: 100, Ri: 100.

Comments: State 1 is found only in Apina and Meliponina.

115. Profurcal pit (modified from Porto et al., in prep.; CHAPTER 2 OF THIS THESIS): (0) present; (1) absent. Ci: 100, Ri: 510.

Comments: State 1 is found only in Apina and Meliponina.

116. Excavation of the profurcal pit (modified from Porto et al., in prep.; CHAPTER 2 OF THIS THESIS): (0) deep, mainly delimited by the basisternal carina; (1) shallow, sometimes with only an incipient basisternal carina restricted to its posterior portion. $\mathrm{Ci}$ : 100, Ri: 100.

Comments: State 1 is found only in Euglossina and Bombina.

117. Connection of the basisternum to the furcasternum (modified from Porto et al., in prep.; CHAPTER 2 OF THIS THESIS): (0) broad; (1) strongly constricted. Ci: 100, Ri: 100.

Comments: State 1 is found only in Apina and Meliponina.

118. Surface of the anterior process of the prosternum (modified from Porto et al., in prep.; CHAPTER 2 OF THIS THESIS): (0) smooth; (1) with a series of well-demarcated transversal lines. Ci: 100, Ri: 100.

Comments: State 1 is found only in Euglossina.

119. Surface of the basisternum (modified from Porto et al., in prep.; CHAPTER 2 OF THIS THESIS): (0) with bristles; (1) smooth. Ci: 50, Ri: 85.

Comments: State 1 is found in Apina and almost all Meliponina. 
120. Surface of the furcasternum (modified from Porto et al., in prep.; CHAPTER 2 OF THIS THESIS): (0) with strong bristles; (1) smooth. Ci: 33, Ri: 60.

Comments: State 1 is found in Apini, Melissodes sp., Diadasina sp., and Tapinotaspoides sp.

121. Median profurcal lamella (modified from Porto et al., in prep.; CHAPTER 2 OF THIS THESIS): (0) forming a single blade; (1) forming a double blade. Ci: 100, Ri: 100.

Comments: State 1 is found only amongst Euglossina.

122. Internal cuticular expansions running along the posterodorsal profurcal apodeme (modified from Porto et al., in prep.; CHAPTER 2 OF THIS THESIS): (0) absent; (1) present. Ci: 100, Ri: 100.

Comments: State 1 is found only amongst Euglossina.

123. Ventral profurcal lamella (modified from Porto et al., in prep.; CHAPTER 2 OF THIS THESIS): (0) strongly produced; (1) weakly produced. Ci: 33, Ri: 80.

Comments: State 1 is found in Apina, Meliponina, Euglossa melanotricha and a few other non-corbiculate taxa.

124. (*) Insertions of the free basal portion of mesofurcal and metafurcal arms to the internal surface of the ventral portion of mesosoma: (0) separated by less than 0.5 times the length of the free basal portion of mesofurcal arm; (1) separated by more than 0.5 times the length of the free basal portion of mesofurcal arm. Ci: 20, Ri: 63.

125. Anterior margin of the mesofurcal bridge (modified from Porto et al., in prep.; CHAPTER 2 OF THIS THESIS): (0) entire; (1) with a somewhat deep median emargination. Ci: 100, Ri: 100 .

Comments: State 1 is found only in Euglossina.

126. Pair of median impressions on the dorsal surface of the mesofurcal bridge sometimes delimiting a broad triangular area (modified from Porto et al., in prep.; CHAPTER 2 OF THIS THESIS): (0) absent; (1) present. Ci: 33, Ri: 60.

Comments: State 0 is found in Acanthopus sp., Apina and most Meliponina. 
127. Round apodemes at the antero-lateral corners of the mesofurcal bridge (modified from Porto et al., in prep.; CHAPTER 2 OF THIS THESIS): (0) absent; (1) present. Ci: 25, Ri: 72.

Comments: State 0 is found in Apina, Meliponina and a few other non-corbiculate taxa.

128. Filamentous apodeme on the dorsal metafurcal lamella (modified from Porto et al., in prep.; CHAPTER 2 OF THIS THESIS): (0) terminal to the sclerotic bar; (1) dislocated laterally in relation to the sclerotic bar. Ci: 14 , Ri: 40 .

129. Mesophragmal longitudinal ridge (modified from Porto et al., in prep.; CHAPTER 2 OF THIS THESIS): (0) present; (1) completely absent. Ci: 50, Ri: 0.

Comments: State 1 is found only in Tapinotaspoides sp. and Tetrapedia maura.

130. Mesophragmal arched ridges (modified from Porto et al., in prep.; CHAPTER 2 OF THIS THESIS): (0) more or less parallel to the antero-posterior axis of the mesophragma (parallel ridges); (1) obliquely oriented in relation to the antero-posterior axis of the mesophragma (oblique ridges); (2) centralized and forming two broad arches on the median area of the mesophragma (arched ridges). Ci: 100, Ri: 100.

Comments: Prentice (1991) has already noted that two broadly arched ridges (state 2) are present on the posterior surface of mesophragma in Bombina, Apina and Meliponina. State 1 is found in Euglossina, Centridini, Anthophora sp. and Acanthopus sp., whereas state 0 is found in the remainder observed taxa.

131. Anterodorsal margin of mesophragma (modified from Porto et al., in prep.; CHAPTER 2 OF THIS THESIS): (0) well-sclerotized, with a more or less regular contour only interrupted medially by the midddorsal notch of mesophragma; (1) feebly sclerotized, with an irregular contour demarcated by several reentrances. Ci: 33, Ri: 66 .

Comments: In the taxa that the anterodorsal margin of mesophragma is feebly sclerotized, it is also expanded into what seems to be equivalent to the pseudophragmal lobes of mesophragma, as already acknowledged by Melo (1999) is his char. 65. State 1 is found in Euglossina, Centridini and Anthophora sp. 
132. Posteroventral margin of mesophragma (modified from Porto et al., in prep.; CHAPTER 2 OF THIS THESIS): (0) fully sclerotized; (1) at least partly membranous. Ci: 50, Ri: 66.

Comments: State 1 is found in Acanthopus sp., Anthophora sp. and Centridini.

133. Middventral notch of mesophragma (modified from Porto et al., in prep.; CHAPTER 2 OF THIS THESIS): (0) absent; (1) present. Ci: 25, Ri: 50.

Comments: State 1 is found in Apina, Bombina, Centris spp. and Anthophora sp.

134. Third phragma (modified from Roig-Alsina \& Michener, 1993: char. 75): (0) much reduced to nearly absent; (1) developed. Ci: 100, Ri: 100.

Comments: Roig-Alsina \& Michener (1993) have stated that the third phragma was absent in Centris tricolor Friese, 1899 and Anthophora paranensis Holmberg, 1903. In this study, the third phragma is interpreted to be present in C. analis, C. flavifrons and Anthophora sp. since it can be distinguished in these taxa. Alternatively, the third phragma in Meliponina is conspicuously reduced, sometimes only indicated by a round spot of feebly sclerotized cuticle on the surface of the propodeum. Thus they are coded as 0 for this character.

135. Third phragma (modified from Roig-Alsina \& Michener, 1993: char. 75): (0) terminating before the marginal area of propodeum; (1) reaching the marginal area of propodeum; (2) extending beyond the marginal area of propodeum. Ci: 50, Ri: 77. 
Appendix 2. Values of the measurements taken to the construction of the continuous characters used in analysis D.

\begin{tabular}{|c|c|c|c|c|c|c|c|c|c|c|c|c|c|c|c|c|c|c|c|c|c|c|c|c|}
\hline \multirow{4}{*}{. } & \multirow{4}{*}{ Taxa } & \multicolumn{23}{|c|}{ Characters } \\
\hline & & \multirow{3}{*}{$\begin{array}{l}\mathbf{0} \\
\mathbf{0} \\
\mathbf{2}\end{array}$} & \multirow{3}{*}{$\begin{array}{l}\mathbf{0} \\
\mathbf{0} \\
\mathbf{3}\end{array}$} & \multirow{3}{*}{$\begin{array}{l}0 \\
0 \\
4\end{array}$} & \multirow{3}{*}{$\begin{array}{l}\mathbf{0} \\
\mathbf{0} \\
9\end{array}$} & \multirow{3}{*}{$\begin{array}{l}\mathbf{0} \\
\mathbf{1} \\
\mathbf{3} \\
\end{array}$} & \multirow{3}{*}{$\begin{array}{l}\mathbf{0} \\
\mathbf{3} \\
\mathbf{6} \\
\end{array}$} & \multirow{3}{*}{$\begin{array}{l}0 \\
4 \\
1\end{array}$} & \multirow{3}{*}{$\begin{array}{l}0 \\
4 \\
2\end{array}$} & \multirow{3}{*}{$\begin{array}{l}0 \\
4 \\
5 \\
\end{array}$} & \multirow{3}{*}{$\begin{array}{l}\mathbf{0} \\
5 \\
2\end{array}$} & \multirow{3}{*}{$\begin{array}{l}0 \\
5 \\
4\end{array}$} & \multirow{3}{*}{$\begin{array}{l}0 \\
5 \\
7 \\
\end{array}$} & \multirow{3}{*}{$\begin{array}{l}0 \\
6 \\
0\end{array}$} & \multirow{3}{*}{$\begin{array}{l}\mathbf{0} \\
6 \\
3 \\
\end{array}$} & \multirow{3}{*}{$\begin{array}{l}0 \\
6 \\
4\end{array}$} & \multirow{3}{*}{$\begin{array}{l}0 \\
6 \\
5\end{array}$} & \multirow{3}{*}{$\begin{array}{l}0 \\
6 \\
7\end{array}$} & \multirow{3}{*}{$\begin{array}{l}\mathbf{0} \\
7 \\
2\end{array}$} & \multirow{3}{*}{$\begin{array}{l}\mathbf{0} \\
7 \\
5\end{array}$} & \multirow{3}{*}{$\begin{array}{l}\mathbf{0} \\
8 \\
2\end{array}$} & 0 & 1 & 1 \\
\hline & & & & & & & & & & & & & & & & & & & & & & 8 & 1 & 2 \\
\hline & & & & & & & & & & & & & & & & & & & & & & 8 & $\mathbf{0}$ & 4 \\
\hline ANT & Anthophora sp. & 3,000 & 1,500 & 0,100 & 0,300 & 0,800 & 0,143 & 8,333 & 5,200 & 0,412 & 1,087 & 0,267 & 0,361 & 0,250 & 1,500 & 0,231 & 1,200 & 0,368 & 0,650 & 0,450 & 0,435 & 5,000 & 1,170 & 0,567 \\
\hline EUC & Melissodes sp. & 2,643 & 0,786 & 0,100 & 0,385 & 0,467 & 0,227 & 3,750 & 5,889 & 1,778 & 0,880 & 0,250 & 0,600 & 0,243 & 0,977 & 0,353 & 2,500 & 0,568 & 0,750 & 0,511 & 0,455 & 3,611 & 1,220 & 0,600 \\
\hline EMP & Diadasina sp. & 3,077 & 0,280 & 1,000 & 0,182 & 0,667 & 0,176 & 4,333 & 4,000 & 0,857 & 0,517 & 0,138 & 0,505 & 0,242 & 1,536 & 0,286 & 1,800 & 0,577 & 0,333 & 0,375 & 0,467 & 7,143 & 1,400 & 0,571 \\
\hline TAP & Tapinotaspoides sp. & 2,333 & 1,333 & 0,100 & 0,455 & 0,472 & 0,273 & 4,643 & 5,909 & 1,167 & 0,667 & 0,185 & 0,489 & 0,163 & 1,700 & 0,160 & 1,000 & 0,213 & 0,833 & 0,511 & 0,455 & 3,056 & 1,220 & 0,833 \\
\hline TET & Tetrapedia maura & 2,000 & 1,333 & 0,333 & 0,200 & 0,714 & 0,333 & 2,000 & 3,385 & 1,222 & 1,000 & 0,132 & 0,474 & 0,189 & 1,429 & 0,260 & 1,444 & 0,156 & 0,400 & 0,286 & 0,364 & 4,444 & 1,700 & 0,667 \\
\hline MAN & Manuelia postica & 2,000 & 2,500 & 1,000 & 0,200 & 0,519 & 0,231 & 3,824 & 4,650 & 0,550 & 1,250 & 0,273 & 0,494 & 0,187 & 1,440 & 0,688 & 4,125 & 0,173 & 0,333 & 0,333 & 0,225 & 7,500 & 1,170 & 0,850 \\
\hline CEN & Epicharis flava & 2,533 & 1,000 & 0,500 & 0,750 & 1,000 & 0,136 & 4,615 & 4,091 & 0,500 & 1,429 & 0,333 & 0,588 & 0,259 & 1,200 & 0,303 & 1,818 & 0,468 & 0,500 & 0,367 & 0,333 & 2,031 & 1,270 & 0,433 \\
\hline CEN & Centris flavifrons & 4,500 & 1,000 & 1,250 & 0,667 & 0,561 & 0,267 & 2,222 & 2,389 & 0,333 & 1,000 & 0,300 & 0,375 & 0,175 & 1,571 & 0,274 & 1,333 & 0,705 & 0,943 & 0,278 & 0,553 & 1,522 & 1,220 & 0,400 \\
\hline CEN & Centris analis & 3,000 & 0,917 & 1,667 & 0,600 & 1,222 & 0,133 & 3,125 & 3,056 & 0,300 & 2,667 & 0,333 & 0,400 & 0,189 & 1,600 & 0,265 & 1,300 & 0,695 & 0,360 & 0,253 & 0,529 & 1,667 & 1,120 & 0,571 \\
\hline EUG & Exaerete smaragdina & 1,400 & 1,000 & 1,400 & 0,500 & 0,667 & 0,100 & 4,167 & 3,889 & 0,412 & 1,786 & 0,262 & 0,660 & 0,200 & 0,680 & 0,529 & 3,364 & 0,100 & 0,250 & 0,400 & 0,118 & 3,571 & 1,020 & 0,520 \\
\hline EUG & Eufriesea violacea & 2,357 & 0,357 & 1,000 & 0,182 & 0,692 & 0,100 & 5,625 & 4,667 & 0,350 & 0,778 & 0,365 & 0,625 & 0,222 & 0,643 & 0,427 & 3,182 & 0,133 & 0,120 & 0,313 & 0,158 & 2,321 & 1,120 & 0,667 \\
\hline EUG & Eulaema nigrita & 1,500 & 0,700 & 1,000 & 0,200 & 0,857 & 0,100 & 5,000 & 5,000 & 0,345 & 1,250 & 0,338 & 0,647 & 0,212 & 0,667 & 0,545 & 2,727 & 0,114 & 0,200 & 0,222 & 0,167 & 2,391 & 1,100 & 0,357 \\
\hline EUG & Euglossa melanotricha & 3,000 & 0,467 & 1,000 & 0,313 & 0,909 & 0,100 & 5,000 & 4,444 & 0,333 & 1,600 & 0,295 & 0,545 & 0,200 & 0,680 & 0,556 & 4,167 & 0,138 & 0,714 & 0,250 & 0,222 & 2,200 & 1,070 & 0,667 \\
\hline $\mathrm{BOM}$ & Bombus pauloensis & 1,333 & 1,000 & 0,100 & 2,059 & 0,327 & 0,222 & 2,000 & 4,583 & 0,438 & 0,833 & 0,615 & 0,493 & 0,167 & 0,741 & 0,533 & 3,556 & $?$ & 0,675 & 0,311 & 0,280 & 3,000 & 1,220 & 0,429 \\
\hline $\mathrm{BOM}$ & Bombus ternarius & 1,000 & 1,000 & 0,100 & 1,400 & 0,333 & 0,235 & 0,769 & 3,632 & 0,587 & 1,000 & 0,479 & 0,475 & 0,168 & 0,750 & 0,447 & 4,200 & $?$ & 0,667 & 0,267 & 0,278 & 2,667 & 1,170 & 0,429 \\
\hline MEL & Dactylurina staudingeri & 1,250 & 0,100 & 0,750 & 0,333 & 0,357 & 0,350 & 0,750 & 2,667 & 0,790 & 0,500 & 0,250 & 0,769 & 0,122 & 1,200 & 0,800 & 6,667 & 0,375 & 0,286 & 0,250 & $?$ & 1,739 & 1,300 & 0,343 \\
\hline MEL & Lophotrigona canifrons & 1,200 & 0,100 & 0,800 & 0,750 & 0,405 & 0,364 & 0,750 & 2,864 & 0,818 & 0,400 & 0,329 & 0,714 & 0,143 & 0,868 & 0,769 & 5,000 & 0,438 & 0,233 & 0,150 & $?$ & 1,765 & 1,270 & 0,435 \\
\hline MEL & Trigonisca nataliae & 0,833 & 0,100 & 0,667 & 1,000 & 0,378 & 0,250 & 0,750 & 3,000 & 0,720 & 0,429 & 0,400 & 0,522 & $?$ & $?$ & $?$ & $?$ & 0,529 & 0,200 & 0,200 & $?$ & 1,857 & 1,420 & 0,467 \\
\hline MEL & Melipona quadrifasciata & 1,176 & 0,300 & 1,333 & 1,000 & 0,326 & 0,286 & 1,000 & 3,167 & 0,563 & 0,625 & 0,325 & 0,563 & 0,163 & 0,900 & 0,706 & 5,000 & 0,639 & 0,233 & 0,158 & $?$ & 2,000 & 1,320 & 0,440 \\
\hline MEL & Tetragonisca angustula & 0,800 & 0,100 & 0,933 & 0,333 & 0,364 & 0,300 & 0,750 & 2,813 & 0,714 & 0,571 & 0,364 & 0,644 & 0,133 & 0,455 & 0,779 & 8,571 & 0,476 & 0,200 & 0,200 & $?$ & 1,739 & 1,420 & 0,450 \\
\hline MEL & Scaptotrigona depilis & 1,000 & 0,100 & 0,667 & 0,333 & 0,315 & 0,357 & 1,000 & 3,176 & 0,357 & 0,444 & 0,375 & 0,643 & 0,121 & 1,000 & 0,648 & 5,833 & 0,472 & 0,182 & 0,158 & $?$ & 1,944 & 1,270 & 0,300 \\
\hline MEL & Trigona hyalinata & 1,154 & 0,300 & 0,769 & 1,000 & 0,300 & 0,333 & 1,500 & 2,917 & 0,625 & 0,400 & 0,371 & 0,789 & 0,097 & 0,926 & 0,923 & 8,571 & 0,418 & 0,167 & 0,220 & $?$ & 2,200 & 1,300 & 0,433 \\
\hline API & Apis dorsata & 1,000 & 0,250 & 0,750 & 1,563 & 0,313 & 0,250 & 1,000 & 2,800 & 0,409 & 5,500 & 0,271 & 0,820 & 0,122 & 0,846 & 0,873 & 4,800 & 0,556 & 0,276 & 0,300 & $?$ & 2,750 & 1,120 & 0,600 \\
\hline API & Apis mellifera & 0,875 & 0,333 & 0,533 & 2,000 & 0,269 & 0,256 & 1,400 & 2,391 & 0,444 & 4,375 & 0,205 & 0,693 & 0,240 & 0,714 & 0,782 & 4,300 & 0,529 & 0,326 & 0,250 & ? & 2,083 & 1,200 & 0,560 \\
\hline
\end{tabular}




\section{General discussion}

The phylogenetic relationships of corbiculate bees and their implications to the comprehension of the evolution of social behaviors in Apidae are certainly one of the most debated topics on bee systematics. Over nearly the past 30 years, since the first works utilizing explicit cladistic methodology to analyze morphological (Winston \& Michener, 1977; Kimsey, 1984; Plant \& Paulus, 1987; Michener, 1990) or molecular datasets (Cameron, 1991, 1993), the accumulation of evidence weighting to alternative groupings - Apina + Meliponina or Meliponina + Bombina - have inflated the longstanding discussions concerning the homology of social structures exhibited by honey bees and stingless bees. On the one hand, the classical works of Michener $(1969,1974)$ have defined the "advanced eusociality" present in Apina and Meliponina as the co-occurrence of (1) cooperative brood care, (2) superposition of generations, (3) division of labor and (4) morphological distinction between castes; on the other hand, Noll (2002) has demonstrated that "eusociality" can be split into more than 40 characters explaining different aspects of social structure (e.g., cell provisioning, nest architecture, swarming, etc.), complemented by approximately 70 characters from Canevazzi \& Noll (2015) describing the variations of hygienic behaviors in Apini.

A recent review by Almeida \& Porto (2014) has also addressed these matters. It was reasoned that the whole discussion about homology of "advanced eusociality" is flawed, since the co-occurrence of the clusters of traits that defined "eusociality" and "advanced eusociality" have appeared at least 12 times in Arthropoda (Wilson \& Hölldobler, 2005; Cardinal \& Danforth, 2011). Thus, a more promising questioning would be to think "advanced eusociality" as an epiphenomenon resultant of the interaction of particular behaviors. Then, as acknowledged by Noll (2002), each behavior must be interpreted as an individual unit that composes the eusocial behaviors. In this context, Almeida \& Porto (2014), following earlier views of Crespi \& Yanega (1994), have suggested that the key element delimiting "primitive" from "advanced" eusociality is the capability of the gyne to found a new colony without the help of workers, which is related to the degree of morphological differentiation between the castes. It was proposed the functional terms "totipotent-caste" and "fixed-caste" eusociality to encompass the gyne potential to found a colony alone or not, in substitution of the teleological definitions such as "primitive" and "advanced" eusociality. Thinking about eusociality this way opens new 
avenues for the understanding of social evolution in Apini. Cardinal \& Danforth (2011) have demonstrated that some of the elements characteristic of eusocial species (1-3) would have been present in the ancestral species of corbiculate bees. These findings were especially interesting if one considers the plasticity found in some species of Euglossa, in which some females share the nest with their sisters (sororal association) or mother (matrifilial association) in the process known as nest-reactivation (i.e., incipient superposition of generations); sometimes the oldest sister/mother acts as the dominant female (i.e., incipient division of labor); and with some instances of cooperative work on cell building (Laroca, 1991; Garófalo et al., 1998; Augusto \& Garófalo, 2009, 2010). The plasticity demonstrated by some species in Euglossina is an indicative that typological definitions as "primitive" and "advanced" are not useful in a phylogenetic paradigm. The best way to understand the social evolution is thinking each behavioral character individually in a phylogenetic context, as it is done for morphological characters. Thus, to properly reconstruct the evolution of the behavioral units constituting the distinct kinds of social structures in Apini, a well-resolved phylogenetic backbone is indispensable.

If the focus of the discussion is changed from the homology/non-homology of behavioral traits to the phylogenetic background necessary to answer these questions, then one obvious problem remaining is the conflict between the evidences from molecular and morphological datasets. As for the molecular studies developed so far, it is clear that the increasing amount of sequences obtained and deposited in the GenBank reflect considerable research efforts in the last two decades to add new data (e.g., Cameron, 1991, 1993; Mardulyn \& Cameron, 1999; Cameron \& Mardulyn, 2001; Kawakita et al., 2008). Morphological datasets, on the other hand, has been restricted by a limited repertoire consisting of repetitions of previous matrices without careful revaluation of the characters proposed. After the work of Roig-Alsina \& Michener (1993), only the work of Cardinal \& Packer (2007) has explored completely novel morphological complexes adding new phylogenetic information to the problem of corbiculate phylogeny.

The internal structures of exoskeleton are an important source of useful morphological information for phylogenetic studies, as has been demonstrated for other hymenopteran taxa (Vilhelmsen, 1996, 1999, 2000a, b, 2011), although poorly explored for bees in general. One obstacle for this kind of study is the lack of appropriate tools to extract information from internal structures, especially from very small specimens. Despite the relative ease to perform scanning 
electron microscopy (SEM) nowadays, other sophisticated methodological alternatives, as MRI, CLSM and $\mu$-CT scan, still are much expensive to execute with larger numbers of specimens (Deans et al., 2012). In this contribution a detailed morphological study of internal structures from head capsule, mandibles, and sitophore was made for a select sample of corbiculate bees. From the 33 characters proposed, more than 15 derived character-states were seen to support the monophyly of Apina-Meliponina-Bombina and Apina-Meliponina clades. Also, detailed descriptions were provided for some of the internal structures of mesosoma - propectus, mesofurca/metafurca and mesophragma - in a comparative study including representatives of all major linages of bees, emphasizing the corbiculate clade. It was demonstrated that the different morphological complexes of mesosoma do not have the same phylogenetic signal, and their significance as sources of information was not equal throughout the phylogeny of bees. When optimized onto distinct phylogenetic hypotheses for bees, the majority of the uncontroverted changes found were concentrated on the prosternum. In this context, the attempt of establishment of a unified morphological terminology for bees, linking to that used for other Hymenoptera, is favorable because it allows that the morphological information gathered in this contribution would be used into broader comparative analyses including the main linages of Hymenoptera. This was pursued making correspondence of specific terms used for bees by previous authors to the ones referenced in the Hymenoptera Anatomy Ontology developed by Yoder et al. (2010), which constitutes an initiative to standardize the current terminology used for Hymenoptera. The morphological information obtained from internal structures was then utilized to construct 42 characters, which in addition to 93 external skeletal characters carefully revaluated and coded from previous authors resulted into a tree congruent with the current morphological/behavioral scenario for the corbiculate relationships: Euglossina $+($ Bombina $+($ Apina + Meliponina $))$. One conclusion that can be made is that characters extracted from internal structures of exoskeleton (e.g., phragma, furcae, apodemes), although poorly explored, provided critical evidence to the issue concerning the "corbiculate controversy", because 18 unique derived character-states supporting groupings into the corbiculate clade were prevenient from internal structures. Another straightforward conclusion is that although the inclusion of new sources of data is indispensable, and the reevaluation of characters currently in (re)use is an obligation for future studies, these alone cannot solve the corbiculate controversy. 
As demonstrated, although the results obtained here points to the traditional hypothesis for corbiculate phylogeny, it is notoriously that many of the evidence that supports the ApinaMeliponina clade are losses/reductions or continuously varying characters. The main sources of unequivocal evidence that supports this group are prosternal modifications. Alternatively, there is also morphological evidence favoring the Meliponina-Bombina grouping, as the unique trilobate apex of the hypopharyngeal lobe of sitophore. Thought the weight of morphological evidence supporting Apina + Meliponina is undoubtedly heavier, this work is not meant to put an endpoint in this discussion. Contrarily, we hope this work encourage further detailed studies with external and internal morphology of bees. One drawback of corbiculate bee morphology is that each subtribe - and tribe as a whole - is morphologically much distinct from other tribes of Apinae. As suggested by Engel (2001), the inclusion of fossil information can be useful to understand what happened during the early stages of the evolution in Apini, as it seems that this group experienced considerable extinction of linages in post-Eocene. This can be one of the factors leading to the observed morphological disparity. In this context, the inclusion of fossil taxa, aided by the use of new technologies for phenotypic studies (Deans et al., 2012) can open new avenues for future comparative morphological studies with corbiculate bees 


\section{References}

Alexander, B. A. (1991a). Phylogenetic analysis of the genus Apis (Hymenoptera: Apidae). Ann. Ent. Soc. Am. 84: 137-149.

Alexander, B. A. (1991b). A cladistic analysis of the genus Apis. In Smith, D. R. (Ed.). Diversity in the Genus Apis (pp. 1-28). Oxford: Westview Press.

Alexander, B. A. \& C. D. Michener (1995). Phylogenetic studies of the families of short-tongued bees (Hymenoptera: Apoidea). Univ. Kansas Sci. Bull. 55: 377-424.

Almeida, E. A. B. \& Porto, D. S. (2014). Investigating Eusociality in Bees while Trusting the Uncertainty. Sociobiology 61(4): 355-368. DOI: 10.13102/sociobiology.v61i4.355-368

Augusto, S. C. \& Garófalo, C. A. (2009). Bionomics and sociological aspects of Euglossa fimbriata (Apidae, Euglossini). Genet. Mol. Res. 8 (2): 525-538. DOI: 10.4238/vol8-2keer004

Augusto, S. C. \& Garófalo, C. A. (2011). Task allocation and interactions among females in Euglossa carolina nests (Hymenoptera, Apidae, Euglossini). Apidologie 42: 162-173. DOI: 10.1051/apido/2010040

Ascher, J. S., Danforth, B. N. \& Ji, S. (2001). Phylogenetic utility of the major opsin in bees (Hymenoptera: Apoidea): a reassessment. Mol. Phylogenet. Evol. 19: 76-93. DOI: 10.1006/mpev.2001.0911

Ascher, J. S. \& Pickering, J. (2014). Discover Life bee species guide and world checklist (Hymenoptera: Apoidea: Anthophila). Available at http://www.discoverlife.org/mp/20q?guide=Apoidea_species. Accessed Nov/15/2014

Berry, R. P. \& Ibbotson, M. R. (2010). A three-dimensional atlas of the honeybee neck. PLoS ONE 5(5): e10771. DOI:10.1371/journal.pone.0010771

Beutel, R. G. \& Vilhelmsen, L. (2007). Head anatomy of Xyelidae (Hexapoda: Hymenoptera) and phylogenetic implications. Organisms, Diversity \& Evolution 7: 207-230. DOI:10.1016/j.ode.2006.06.003

Bohart, R. M. \& Menke, A. S. (1976). Sphecid Wasps of the World. Berkeley: University of California Press.

Bremer, K. (1994). Branch support and tree stability. Cladistics 10: 295-304. DOI: 10.1111/j.10960031.1994.tb00179.x

Brothers, D. J. (1975). Phylogeny and classification of the aculeate Hymenoptera, with special reference to Mutilidae. Univ. Kansas Sci. Bull. 50: 483-648.

Burks, R.A. \& Heraty, J.M. (2015) Subforaminal bridges in Hymenoptera (Insecta), with a focus on Chalcidoidea. Arthropod Struct. Dev. 44: 173-194. DOI:10.1016/j.asd.2014.12.003 
Camargo, J. M. F., Kerr, W. E. \& Lopes, C. R. (1967). Morfologia externa de Melipona (Melipona) marginata Lepeletier (Hymenoptera, Apoidea). Pap. Avulsos Zool., São Paulo 20: 229-258.

Camargo, J. M. F. \& Pedro, S. R. M. (1992). Systematics, phylogeny and biogeography of the Meliponinae (Hymenoptera: Apidae): a mini-review. Apidologie 23: 509-522. DOI: 10.1051/apido: 19920603

Camargo, J. M. F. \& Pedro, S. R. M. (2013). Meliponini Lepeletier, 1836. In Moure, J. S., Urban, D. \& Melo, G. A. R. (Orgs). Catalogue of Bees (Hymenoptera, Apoidea) in the Neotropical Region online version. Available at http://www.moure.cria.org.br/catalogue. Accessed Nov/15/2014

Cameron, S. A. (1991). A new tribal phylogeny of the Apidae inferred from mitochondrial DNA sequences. In Smith, D. R. (Ed.), Diversity in the Genus Apis (pp. 71-87). Oxford: Westview Press.

Cameron, S. A. (1993). Multiple origins of advanced eusociality in bees inferred from mitochondrial DNA sequences. Proc. Natl. Acad. Sci. USA 90: 8687-8691.

Cameron, S. A. (2004). Phylogeny and biology of Neotropical orchid bees (Euglossini). Annu. Rev. Entomol. 49: 377-404. DOI: 10.1146/annurev.ento.49.072103.115855

Cameron, S. A. \& Mardulyn, P. (2001). Multiple molecular data sets suggest independent origins of highly eusocial behavior in bees (Hymenoptera: Apinae). Syst. Biol. 50 (2): 194-214. DOI: 10.1080/10635150120230

Cane, J. H. (1979). The hind tibiotarsal and tibial spur articulations in bees. J. Kansas Entomol. Soc. 52: 123-137.

Cardinal, S. \& Danforth, B. N. (2011). The antiquity and evolutionary history of social behavior in bees. PLoS ONE 6(6): e21086. DOI: 10.1371/journal.pone.0021086

Cardinal, S. \& Danforth, B. N. (2013). Bees diversified in the age of eudicots. Proc. R. Soc. B. 280: 20122686. DOI: $10.1098 / \mathrm{rspb} .2012 .2686$

Cardinal, S. \& Packer, L. (2007). Phylogenetic analysis of corbiculate Apinae based on morphology of the sting apparatus (Hymenoptera: Apidae). Cladistics 23: 99-118. DOI: 10.1111/j.10960031.2006.00137.x

Cardinal, S., Straka, J. \& Danforth, B. N. (2010). Comprehensive phylogeny of apid bees reveals the evolutionary origins and antiquity of cleptoparasitism. Proc. Nat. Acad. Sci. USA 107: 1620716211. DOI: 10.1073/pnas.1006299107

Canevazzi, N. C. S. \& Noll, F. B. (2015). Cladistic analysis of self-grooming indicates a single origin of eusociality in corbiculate bees (Hymenoptera: Apidae). Cladistics 31(2): 126-141. DOI: 10.1111/cla.12077

Chavarría, G. \& Carpenter, J. M. (1994). “Total evidence” and evolution of highly social bees. Cladistics 10: 229-258. DOI: 10.1111/j.1096-0031.1994.tb00177.x 
Crespi, B. J. \& Yanega, D. (1994). The definition of eusociality. Behav. Ecol. 6: 109-115. DOI: 10.1093/beheco/6.1.109

Cruz-Landim, C. (1967). Estudo comparativo de algumas glândulas das abelhas (Hymenoptera, Apoidea) e respectivas implicacões evolutivas. Arq. Zool. S. Paulo 15: 177-290.

Danforth, B. N., Cardinal, S., Praz, C., Almeida, E. A. B. \& Michez, D. (2013). The impact of molecular data on our understanding of bee phylogeny and evolution. Annu. Rev. Entomol. 58: 57-78. DOI: 10.1146/annurev-ento-120811-153633

Deans, A. R., Mikó, I., Wipfler, B. \& Friedrich, F. (2012). Evolutionary phenomics and the emerging enlightenment of arthropod systematics. Invertebr. Syst. 26: 323-330 DOI: 10.1071/IS12063

Engel, M. S. (1999). The first fossil Euglossa and phylogeny of the orchid bees (Hymenoptera: Apidae: Euglossini). Am. Mus. Novit. 3272:1-14

Engel, M. S. (2001a). A monograph of the Baltic amber bees and evolution of the Apoidea (Hymenoptera). Bull. Am. Mus. Nat. Hist. 259: 1-192. DOI: dx.doi.org/10.1206/00030090(2001)259<0001:AMOTBA>2.0.CO;2

Engel, M. S. (2001b). Monophyly and extensive extinction of advanced eusocial bees: Insights from an unexpected Eocene diversity. Proc. Natl Acad. Sci. USA 98: 1661-1664. DOI: 10.1073/pnas.98.4.1661

Engel, M. S. (2005). Family-group names for bees (Hymenoptera: Apoidea). American Museum Novitates 3476: 1-33. DOI: 10.1206/0003-0082(2005)476[0001:FNFBHA]2.0.CO;2

Engels, W. \& Imperatriz-Fonseca, V. L. (1990). Caste development, reproductive strategies and control of fertility in honey bees and stingless bees. In Engels, W. (Ed.), Social Insects: An Evolutionary Approach to Caste and Reproduction (pp. 167-230). Heidelberg: Springer Berlin.

Faustino, C. D., Silva-Matos, E. V., Mateus, S. \& Zucchi, R. (2002). First record of emergency queen rearing in stingless bees (Hymenoptera, Apinae, Meliponini), Insectes Soc. 49: 111-113. DOI: 10.1007/s00040-002-8287-x

Felsenstein, J. (1985). Confidence limits on phylogenies: an approach using the bootstrap. Evolution 39: 783-791.

Garófalo, C. A., Camillo, E., Augusto, S. C., Jesus, B. M. V. \& Serrano, J. C. (1998). Nest structure and communal nesting in Euglossa (Glossura) annectans Dressler (Hymenoptera, Apidae, Euglossini). Revta. Bras. Zool. 15(3): 589-596.

Greco, M. K., Welz, P. M., Siegrist, M., Ferguson, S. J., Gallmann, P., Roubik, D. W. \& Engel, M. S. (2011). Description of an ancient social bee trapped in amber using diagnostic radioentomoly. Insect. Soc. DOI: 10.1007/s00040-011-0168-8 
Goloboff, P. A., J. S. Farris, M. Källersjö, B. Oxelman, M. J. Ramırez \& C. A. Szumik (2003). Improvements to resampling measures of group support. Cladistics 19: 324-332. DOI: 10.1111/j.1096-0031.2003.tb00376.x

Goloboff, P. A., Mattoni, C. I. \& Quinteros, A. S. (2006). Continuous characters analyzed as such. Cladistics 22:589-601. DOI: 10.1111/j.1096-0031.2006.00122.x

Goloboff, P. A., Farris, J. S. \& Nixon, K. C. (2008). TNT, a free program for phylogenetic analysis. Cladistics 24: 774-786. DOI: 10.1111/j.1096-0031.2008.00217.x

Goloboff, P. A. (1993). Estimating character weights during tree search. Cladistics 9: 83-91. DOI: 10.1111/j.1096-0031.1993.tb00209.x

Hines, H. M. (2008). Historical biogeography, divergence times, and diversification patterns of bumble bees (Hymenoptera: Apidae: Bombus). Syst. Biol. 57(1): 58-75. DOI: $10.1080 / 10635150801898912$

Imperatriz-Fonseca, V. L. \& Zucchi R. (1995). Virgin queens in stingless bee (Apidae, Meliponinae) colonies: a review. Apidologie 26: 231-244. DOI: 10.1051/apido:19950305

Karlsson, D. \& Ronquist, F. (2012). Skeletal morphology of Opius dissitus and Biosteres carbonarius (Hymenoptera: Braconidae), with a discussion of terminology. PLoS ONE 7(4): e32573. DOI:10.1371/journal.pone.0032573

Kawakita, A., Ascher, J. S., Sota, T., Kato, M. \& Roubik, D. W. (2008). Phylogenetic analysis of the corbiculate bee tribes based on 12 nuclear protein-coding genes (Hymenoptera: Apoidea: Apidae). Apidologie 39: 163-175. DOI: 10.1051/apido:2007046

Kerr, W. E. (1969). Some aspects of the evolution of social bees (Apidae). Evol. Biol. 3: 119-175.

Kerr, W. E. (1987). Sex determination in bees. XVII. Systems of caste determination in the Apinae, Meliponinae and Bombinae and their phylogenetic implications. Rev. Bras. Genet. 10: 685-694.

Kimsey, L. S. (1984). A re-evaluation of the phylogenetic relationships in the Apidae (Hymenoptera). Syst. Entomol. 9: 435-441. DOI: 10.1111/j.1365-3113.1984.tb00519.x

Koulianos, S., Schmid-Hempel, R., Roubik, D. W. \& Schmid-Hempel, P. (1999). Phylogenetic relationships within the corbiculate Apinae (Hymenoptera) and the evolution of eusociality. J. Evol. Biol. 12: 380-384.

Laroca, S. (1991). Euglossa stellfeldi: Arquitetura do ninho e coexistência com Pseudocentron apicipennis em uma mesma cavidade. Acta Biol. Par. 20: 103-108.

Lockhart, P. G. \& Cameron, S. A. (2001). Trees for bees. Trends Ecol. Evol. 16: 84-88. DOI: http://dx.doi.org/10.1016/S0169-5347(00)02054-1

Maa, T. (1953). An inquiry into the systematics of the tribus Apidini or honeybees (Hym.). Treubia 21: 525-640. 
Mardulyn, P. \& Cameron, S. A. (1999). The major opsin in bees (Insecta: Hymenoptera): a promising nuclear gene for higher level phylogenetics. Mol. Phylogenet. Evol. 12: 168-176. DOI: 10.1006/mpev.1998.0606

Martins, A. C., Melo, G. A. R. \& Renner, S. S. (2014). The corbiculate bees arose from New World oilcollecting bees: Implications for the origin of pollen baskets. Molec. Phylogen. Evol. 80, 88-94. DOI: 10.1016/j.ympev.2014.07.003

Melo, G. A. R. (1999). Phylogenetic relationships and classification of the major lineages of Apoidea (Hymenoptera), with emphasis on crabronid wasps. Sci. Papers 14: 1-55.

Michel-Salzat, A., Cameron, S. A. \& Oliveira, M. L. (2004). Phylogeny of the orchid bees (Hymenoptera: Apinae: Euglossini): DNA and morphology yield equivalent patterns. Mol. Phylogenet. Evol. 32(1), 309-323. DOI:10.1016/j.ympev.2003.12.009

Michener, C. D. (1944). Comparative external morphology, phylogeny, and a classification of the bees (Hymenoptera). Bull. Am. Mus. Nat. Hist. 82: 151-326.

Michener, C. D. (1974). The Social Behavior of the Bees: A Comparative Study. Cambridge: Belknap, $404 \mathrm{p}$.

Michener, C. D. (1981). Comparative morphology of the middle coxae of Apoidea. J. Kansas Entomol. Soc. 54(2): 319-326.

Michener, C. D. (1984). A comparative study of the mentum and lorum of bees. J. Kansas Entomol. Soc. 57: 705-7014.

Michener, C. D. (1990). Classification of Apidae (Hymenoptera). Univ. Kansas Sci. Bull. 54: 75-164.

Michener, C. D. (2007). The Bees of the World ( $2^{\text {nd }}$ Ed). Baltimore: John Hopkins University Press, 953 p.

Michener, C. D. \& Brooks, R. W. (1984). Comparative study of the glossae of bees. Contributions of the American Entomological Institute 22(1): i-iii+1-73.

Michener, C. D. \& Fraser, A. (1978). A comparative anatomical study of mandibular structures in bees. Univ. Kansas Sci. Bull. 51: 463-482.

Moure, J. S., Melo, G. A. R. \& Faria, L. R. R., Jr. (2012). Euglossini Latreille, 1802. In Moure, J. S., Urban, D. \& Melo, G. A. R. (Orgs). Catalogue of Bees (Hymenoptera, Apoidea) in the Neotropical Region - online version. Available at http://www.moure.cria.org.br/catalogue. Accessed Nov/15/2014

Nixon, K. C. (2002). Winclada, v. 1.00. 08. Program and documentation available at www. cladistics.com.

Noll, F. B. (2002). Behavioral phylogeny of corbiculate Apidae (Hymenoptera; Apinae), with special reference to social behavior. Cladistics 18: 137-153. DOI: 10.1006/clad.2001.0191 
Packer, L. (2003). Comparative morphology of the skeletal parts of the sting apparatus of bees (Hymenoptera: Apoidea). Zoolog. J. Linn. Soc. 138: 1-38. DOI: 10.1046/j.10963642.2003.00055.x

Packer, L. (2004). Morphological variation in the gastral sterna of female Apoidea (Insecta: Hymenoptera). Canad. J. Zool 82: 130-152. DOI: 10.1139/z03-196

Payne, A. (2013). Resolving the relationships of apid bees (Hymenoptera: Apidae) through a direct optimization sensitivity analysis of molecular, morphological, and behavioral characters. Cladistics: 1-15. DOI: 10.1111/cla.12022

Peixoto, E. B. M. I. \& Serrão, J. E. (2001). A comparative study of the cardia and cardiac valves in the corbiculate bees (Hymenoptera: Apinae). Sociobiology 37: 707-721.

Pereira-Martins, S. R. \& Kerr, W. E. (1991). Biologia de Eulaema nigrita. 1. Construção de células, oviposição e desenvolvimento. Pap. Avuls. Zool. 37: 227-235.

Plant, J. D. \& Paulus, H. F. (1987). Comparative morphology of the postmentum of bees (Hymenoptera: Apoidea) with special remarks on the evolution of the lorum. Z. Zool. Syst. Evol. 25: 81-103.

Porto, D. S., Vilhelmsen, L., \& Almeida, E. A. (2015). Comparative morphology of the mandibles and head structures of corbiculate bees (Hymenoptera: Apidae: Apini). Syst. Entomol.

Prentice, M. (1991). Morphological analysis of the tribes of Apidae. In Smith, D. R. (Ed.). Diversity in the Genus Apis (pp. 51-69). Oxford: Westview Press.

Ramírez, S. R., Roubik, D.W., Skov, C. \& Pierce, N. E. (2010). Phylogeny, diversification patterns and historical biogeography of euglossine orchid bees (Hymenoptera: Apidae). Biol. J. Linn. Soc. 100: 552-572. DOI: 10.1111/j.1095-8312.2010.01440.x

Rasmussen, C. \& Cameron, S. A. (2010). Global stingless bee phylogeny supports ancient divergence, vicariance, and long distance dispersal. Biol. J. Linn. Soc. 99: 206-232. DOI: 10.1111/j.10958312.2009.01341.x

Rightmyer, M.G. (2004). Phylogeny and classification of the parasitic bee tribe Epeolini (Hymenoptera: Apidae, Nomadinae). Scientific Papers, Natural History Museum, The University of Kansas, 33, 151.

Roig-Alsina, A. \& Michener, C. D. (1993). Studies of the phylogeny and classification of long-tongued bees (Hymenoptera: Apoidea). Univ. Kansas Sci. Bull. 55: 123-160.

Sakagami S. F. (1982). Stingless bees. In Hermann H.R. (Ed.), Social insects (pp. 361-423). New York: Academic Press.

Santos-Filho, P. de S., Alves, D. de A., Eterovic, A., Imperatriz-Fonseca, V. L. \& Kleinert, A. de M. P. (2006). Numerical investment in sex and caste by stingless bees (Apidae: Meliponini): a comparative analysis. Apidologie 37: 207-221. 
Schönitzer, K. (1986). Comparative morphology if the antenna cleaner in bees (Apoidea). Z. Zool. Syst. Evol. 24: 35-51.

Schultz, T. R., Engel, M. S. \& Prentice, M. (1999). Resolving conflict between morphological and molecular evidence for the origin of eusociality in the "corbiculate" bees (Hymenoptera: Apidae): a hypothesis-testing approach. Uni. Kans. Nat. Hist. Mus. Spec. Publ. 24: 125-138.

Serrão, J. E. (2001). A comparative study of the proventricular structure in corbiculate apinae (Hymenoptera: Apidae). Micron 32: 379-385. DOI: 10.1016/S0968-4328(00)00014-7

Sheppard, W. S. \& McPheron, B. A. (1991). Ribosomal DNA diversity in Apidae. In Smith, D. R. (Ed.), Diversity in the genus Apis (pp. 89-102). Oxford: Westview Press.

Silveira, F. A., Melo, G. A. R. \& Almeida, E. A. B. (2002a). Abelhas Brasileiras: Sistemática e Identificação. Belo Horizonte: F. A. Silveira, 253 pp.

Snodgrass, R. E. (1910). The Anatomy of the Honey Bee. Washington: Government Printing Office, 236 p.

Snodgrass, R. E. (1935). Principles of insect morphology. New York: McGraw-Hill Book Co., ix + 667 p.

Snodgrass, R. E. (1942). The skeleto-muscular mechanisms of the honey bee. Smithson. misc. Coll. 103(2): 1-120.

Snodgrass, R. E. (1956). Anatomy of the Honey Bee. Ithaca: Cornell University Press., i-xiv + 334 p.

Soucy, S. L., Giray, T. \& Roubik, D. W. (2003). Solitary and group nesting in the orchid bee Euglossa hyachintina (Hymenoptera, Apidae). Insect. Soc. 50: 248-255. DOI: 10.1007/s00040-003-0670-8

Tarpy, D. R. \& Gilley. D.C. (2004). Group decision making during queen production in colonies of highly eusocial bees. Apidologie 35: 207-216. DOI: 10.1051/apido:2004008

Tóth, E., Queller, D. C., Dollin, A. \& Strassmann, J. E. (2004). Conflict over male parentage in stingless bees. Insect. Soc. 51: 1-11. DOI: 10.1007/s00040-003-0707-z

Vilhelmsen, L. (1996). The preoral cavity of lower Hymenoptera (Insecta): comparative morphology and phylogenetic significance. Zool. Scr. 25(2): 143-170. DOI: 10.1111/j.1463-6409.1996.tb00156.x

Vilhelmsen, L. (1999). The occipital region in the basal Hymenoptera (Insecta): a reappraisal. Zool. Scr. 28: 75-85. DOI: 10.1046/j.1463-6409.1999.00008.x

Vilhelmsen, L. (2000a). Before the wasp-waist: comparative anatomy and phylogenetic implications of the skeleto-musculature of the thoraco-abdominal boundary region in basal Hymenoptera (Insecta). Zoomorphology 119: 185-221. DOI: 10.1007/PL00008493

Vilhelmsen, L. (2000b). Cervical and prothoracic skeleto-musculature in the basal Hymenoptera (Insecta): comparative anatomy and phylogenetic implications. Zool. Anz. 239: 105-138. 
Vilhelmsen, L. (2000c). The ovipositor apparatus of basal Hymenoptera (Insecta): phylogenetic implications and functional morphology. Zool. Scr. 29(4): 319-345. DOI: 10.1046/j.14636409.2000.00046.x

Vilhelmsen, L. (2011). Head capsule characters in the Hymenoptera and their phylogenetic implications. ZooKeys 130. 343-361. DOI: 10.3897/zookeys.130.1438

Vilhelmsen, L., Mikó, I. \& Krogmann, L. (2010). Beyond the wasp-waist: structural diversity and phylogenetic significance of the mesosoma in apocritan wasps (Insecta: Hymenoptera). Zool. J. Linn. Soc. Lond. 159: 22-194. DOI: 0.1111/j.1096-3642.2009.00576.x

Vilhelmsen L. \& Zimmermann, D. (2014). Baltorussus Total Makeover: Rejuvenation and Sex Change in an Ancient Parasitoid Wasp Lineage. PLoS ONE 9(6):

e98412. doi:10.1371/journal.pone.0098412

Whitfield, J. B. \& Kjer, K. M. (2008). Ancient Rapid Radiations of Insects: Challenges for Phylogenetic Analysis. Annu. Rev. Entomol. 53: 23.1-23.4 DOI: 10.1146/annurev.ento.53.103106.093304

Wilson, E. O. \& Hölldobler, B. (2005). Eusociality: Origin and consequences. Proc. Natl. Acad. Sci. USA 102(38):13367-13371. DOI: 10.1073/pnas.0505858102

Winston, M.L. (1979). The proboscis of the long-tongued bees: A comparative study. University of Kansas Science Bulletin 51: 631-667.

Winston, M. L. \& Michener, C. D. (1977). Dual origin of highly social behavior among bees. Proc. Natl. Acad. Sci. USA 74: 1135-1137.

Yoder M. J., Mikó I., Seltmann K. C., Bertone, M. A. \& Deans, A. R. (2010). A Gross Anatomy Ontology for Hymenoptera. PLoS ONE 5 (12): e15991. DOI: 10.1371/journal.pone.0015991. 


\title{
Appendix 1 - Investigating Eusociality in Bees while Trusting the Uncertainty
}

\section{E A B ALMEIDA ${ }^{1}$, D S PORTO}

Laboratório de Biologia Comparada e Abelhas (LBCA), Departamento de Biologia. Faculdade de Filosofia, Ciências e Letras de Ribeirão Preto (FFCLRP). Universidade de São Paulo. Avenida Bandeirantes, 3900. 14040-901, Ribeirão Preto-SP, Brazil.

${ }^{1}$ Corresponding author: eduardo@ffclrp.usp.br

Running title: Eusociality in Corbiculate Bees

Keywords: Apini, social behavior, Comparative Biology, divergence time, Meliponini, phylogeny

Original content distributed under the Creative Commons Attribution License (CC BY 3.0). Originally published by Universidade Estadual de Feira de Santana: Almeida, E. A. B. \& Porto, D. S. (2014). Investigating Eusociality in Bees while Trusting the Uncertainty. Sociobiology 61(4): 355-368. DOI: 10.13102/sociobiology.v61i4.355-368

\begin{abstract}
Phylogenetic hypotheses and estimates of divergence times have already been used to investigate the evolution of social behavior in all lineages of bees. The interpretation of the number of origins of eusocial behavior and the timing of these events depends on reliable phylogenetic hypotheses for the clades in which these lineages are nested. Three to six independent origins of eusocial behavior are interpreted to have occurred in bee taxa that differentiated in the Late Cretaceous, or much later in the Paleogene. Only two groups of bees exhibit the behaviors that qualify their members to be considered obligate (i.e. 'fixed-caste') eusocial, the honey bees (Apini) and the stingless bees (Meliponini). The evolutionary history of corbiculate bees remains uncertain in many respects, but phylogenetic research has been
\end{abstract}


paving the path for comprehensive comparative approaches likely to shed light on the origin of diversity of forms and behaviors of these bees. In total, corbiculate bees encompass about 1,000 species, roughly $5 \%$ of the described species diversity of bees. These bees are rather heterogeneous in terms of social organization, particularly stingless bees and orchid bees, which display a fascinating range of behavioral variation. Using phylogenetic tools, it has been possible to infer that caste polymorphism, division of labor and other traits of corbiculate bees probably started evolving over 80 million years ago. Phylogenetic hypotheses must be interpreted as more or less uncertain scenarios for studying the biological diversity, but when trusted they can provide powerful tools to investigate the evolution of social behaviors.

\section{Introduction}

During the last century, research on bee behavior and morphology has largely become increasingly comparative, incorporating the diversity of traits found in the variety of species of these insects (e.g., Michener, 1944, 1974, 2007; Engel, 2011; Danforth et al. 2013). Remarkably detailed investigations had been previously made on bees (e.g., Schmid \& Kleine, 1861; Girdwoyn, 1876; Macloskie, 1881; Briant, 1884; Carlet, 1884; Bordas, 1894; Abonyi, 1903; Hommell, 1904, 1905; Arnhart, 1906; Snodgrass, 1910, 1935, 1942, 1956), particularly with Apis mellifera Linnaeus, 1758 - the honeybee. This has long been a species with close proximity of mankind (the species of Apis are distributed all over the Old World), with economic interest because of honey production (pollination became a hotly debated topic more recently, e.g., Oldroyd, 2007; Cameron et al., 2010; Potts et al., 2010; Albrecht et al., 2012; Bartolomeus et al., 2013; Garibaldi et al., 2013), and certainly because of its fascinating social organization. Along with ants, bumble bees, termites, social wasps, and unusual species of mammals (mole-rats: Jarvis [1981]), honey bees have had a prime importance for the understanding of the cohesion observed among organisms of some species that allow them to live close together, interact, share tasks related to survival and reproduction. Arthropods comprise about half of the described biodiversity, and the total number of known origins of "eusociality" is approximately 12 in this clade (Wilson, 1971; Wilson \& Hölldobler, 2005). Sociality, in this way, is a very rare condition in nature, indicating that it is not a frequently acquired trait, particularly because it involves reproductive division of labor (Wilson, 1971) 
and all potential conflicts related to this (e.g., Trivers \& Hare, 1976; Reeve \& Keller, 1999; Tóth et al., 2004).

If the evolution of sociality was elementary, one might ask why there are relatively few eusocial species. Although the number of social species is reduced in comparison to the known biodiversity, sociality can be viewed as ubiquitous because social organisms, particularly insects, are everywhere and tend to be very common. Some estimates indicate that social organisms, particularly termites and ants, might comprise almost $30 \%$ of the biomass of a tropical rainforest (Fittkau \& Klinge, 1973); and that the number of workers in a single colony of ants may reach more than 20 million (Hölldobler \& Wilson, 1990; Wilson \& Hölldobler, 2005). Among termites, this number can be as high as 13 million individual workers (Lee, 2002), honey bees are documented to have colonies comprising between 10 to 100 thousand workers (Bourke, 1999) and nests of eusocial stingless bees of the genus Scaptotrigona (Meliponini) may encompass 50 thousand workers (Lindauer \& Kerr, 1960). These huge colonies account for the ubiquity and the heavy biomass of social organisms.

The study of the honey bee and research of all the traits related to its social behavior have been a challenge posed by researchers of several fields over the centuries. In addition to external and internal morphology (e.g., Snodgrass, 1910, 1942, 1956), there were several landmarks in ethological investigation made with A. mellifera (the most famous must be Karl von Frisch's 1946 study that deciphered the "waggle dance" of honey bees — see also the excellent summaries by Winston, 1987; Seeley, 1995, 2010; Page, 2013); genetics (A. mellifera mitochondrial genome sequencing [Crozier \& Crozier, 1993]; discovery of important insulin/TOR signaling pathways and receptors related to queen caste determination [Patel et al., 2007]; discovery of the royalactin by Kamakura, 2011]); physiology (food contents versus caste determination in A. mellifera; hormones and pathways related to social behaviors, queen dominance, etc. [e.g. Weaver, 1955; Laidlaw et al., 1956; Hartfelder \& Engels, 1998; Hartfelder, 2000; Sullivan et al., 2000]); and more recently genomics (Honeybee Genome Sequencing Consortium, 2006).

A natural progress in the research of social behavior is to make it comparative, to unravel similarities and differences among the diversity of organisms that display some level of sociality. There exist intricate physiological (hormonal/ neurobiological) interactions of different signaling pathways that accounts for the complexly self-organized colony of $A$. 
mellifera, perhaps just a fortuitous result of the evolutionary odds, perhaps a set of specific mechanistic components interacting with each other, responsible for ordering colony-level functions. One of the exciting challenges resulting from the enormous body of knowledge about A. mellifera is the understanding of how social interactions would be expressed in other bee species that also exhibit complex behaviors related to the self-organization of their societies (e.g., Page, 2013), and how comparative research could help elucidate the evolution of social behaviors.

\section{Eusociality}

Among the various levels of intraspecific interactions that can be interpreted as social interactions, this work will focus on the eusocial behavior of bees, more specifically on obligate eusociality sensu Crespi and Yanega (1994; commonly referred to as "advanced eusocial" or "highly eusocial" behavior). Michener (1974) presented a comprehensive classification of kinds of social behaviors documented among bees in his chapter 5 "Kinds of Societies among Bees". Eusocial organisms may be characterized by life in "colonies, which are family groups", "consisting of individuals of two generations, mothers and daughters"; and by "division of labor, with some individuals functioning as egg layers or queens and others as workers, that is with more or less recognizable castes" (Michener, 1974: p.46). "Eusociality" has been defined in slightly different ways over the years, but the presence of castes seems to be a main trait for the recognition of this phenomenon (Crespi \& Yanega, 1994). Other behaviors that are often cited as necessarily co-occurring in eusocial organisms are the superposition of generations and the cooperation in the tasks necessary for survival and reproduction. One important distinction between eusocial bees refers to the morphological polymorphism between females of reproductive and non-reproductive castes, and the capacity of gynes (i.e., potential or actual reproductive individual: queen) of surviving alone (Michener, 1974). When the morphological distinction is more clearly marked and a gyne is not able to start a nest by herself (Michener 1974, 2007), hence neither caste can be said to be totipotent (Crespi \& Yanega, 1994), there is a phenomenon defined as dependent colony foundation, which was neatly reviewed by Cronin et al. (2013). Lack of gyne totipotency thus is key for the recognition of "advanced eusocial" or "highly eusocial", or "obligate eusocial" behavior. In this paper we choose to favor the term "fixed-caste eusociality", similar to that proposed by 
Crespi and Yanega (1994), because it transmits a less teleological notion than the former two. Michener (1974: p.47) remarked that it "is tempting to look at the sequence of increasingly complex societies (...) as an evolutionary sequence", but in fact multiple evolutionary changes toward simpler as well as more complex behaviors have occurred throughout bee history. Terms with ability to introduce misconceptions, such as "primitive" and "advanced" should, hence, be avoided and replaced by "totipotent-caste" and "fixed-caste" when possible. There are at least five independent origins of eusociality among bees, as discussed below, but obligate eusociality is rare and can only be found in the honey bees (Apini: Apis) and the stingless bees (Meliponini).

\section{Phylogenetic research and the understanding of social evolution}

Recent insights given by phylogenetic work have allowed estimation of divergence ages between distinct lineages of social organisms (e.g., Cardinal \& Danforth, 2011). The two most distantly related eusocial taxa, mammals and insects, diverged between the Precambrian and the Early Cambrian, at the split of the common ancestor of bilaterian animals that gave rise to Protostomia and Deuterostomia (Blair, 2009; Edgecombe et al., 2011). Focusing on the eusocial insects, termites diverged from the lineage that gave rise to Hymenoptera in the beginning of the Mesozoic or earlier (Ware et al., 2010; Cardinal \& Danforth, 2011), and began their differentiation and diversification about 235 Mya, during the Late Triassic. Within Hymenoptera the origin of eusocial behavior in ants probably occurred in the early Cretaceous, which occurred independently from vespid wasps (some time in the Cretaceous: Grimaldi \& Engel [2005], Brady et al. [2006a], Moreau et al. [2006], Hines et al. [2007]); and bees, which comprise three to six independent origins of eusocial behavior in taxa that differentiated in the Late Cretaceous, or much later in the Paleogene (Brady et al., 2006b; Chenoweth et al., 2007; Cardinal \& Danforth, 2011; Gibbs et al., 2012; Danforth et al., 2013).

These discrepancies in divergence ages bring one certainty and several possible questions. We can say we are confident that eusociality is not a homologous condition that can be properly used in a comparative (i.e., historical) context. Therefore, caution must be exercised at all times when comparisons are made between taxa showing social behaviors because their traits may not be directly comparable (i.e., homologous; Nixon \& Carpenter, 2012). A more radical interpretation of sociality, as a non-homologous trait, is its 
interpretation as an emerging property or epiphenomenon resulting from the co-occurrence of complex suite of traits related to the coexistence and certain interactions among individuals of a species. Given that eusociality surely is not a homologous characteristic, there are various interesting queries that can be posed about the evolution of social behaviors, such as the following: (a) When can we assume that at least some of the traits comprised in the definition of "eusociality" to be homologous?; (b) Do the defining traits of "eusociality" appear together or they evolve in a stepwise manner?; (c) Is it possible to relate multiple evolution of "eusocial" behaviors with general evolutionary factors or they are mostly intrinsic properties of the organisms that possess them?

It is very possible that many of those questions will be confidently answered in the years to come partly because of the empirical knowledge that is fundamental for depiction of life's diversity, as well as the growing phylogenetic basis necessary for historical comparative research. Below we are going to discuss the relative confidence and uncertainty regarding the phylogenetic history of bees (and its relevance for comparative research); the most diverse taxon of obligate eusocial bees: the Meliponini; and the importance of the growing body of knowledge about orchid bees to the understanding of behaviors in corbiculate bees.

\section{Relative Uncertainty of Phylogenetic Hypotheses Available for Bees}

Our understanding of bee phylogeny has been improving (Michener, 1944, 2007; RoigAlsina \& Michener, 1993; Alexander \& Michener, 1995; Danforth et al., 2013) and, with it, a growing comparative framework for understanding the evolution of social behavior is being constructed. The higher-level (i.e., family-level) classification of bees has become very remarkably stable over the last decades particularly because of intense phylogenetic research within Apoidea (e.g., Roig-Alsina \& Michener, 1993; Alexander \& Michener, 1995; Melo, 1999; Danforth et al., 2006a, b, 2013; Michener, 2007; Engel 2011; Debevec et al., 2012; Cardinal \& Danforth 2013; Hedke et al., 2013). There are still portions of the tree of bees that can be represented by incongruent competing hypotheses (e.g., Alexander \& Michener, 1995; Engel, 2011; Danforth et al., 2013), but the accumulated scientific progress is nevertheless clear. Phylogenetic hypotheses and estimates of divergence times have been used to investigate the evolution of social behavior in all lineages of bees (reviewed by Schwarz et al., 2007; Cardinal \& Danforth, 2011; Danforth et al., 2013). The interpretation of the number of 
origins of eusocial behavior and the timing these events depends on reliable phylogenetic hypotheses for the clades in which these lineages are nested. A summary of bee phylogeny is presented in Figure 1, depicting in particular detail clades where eusocial behavior is present in all of its component taxa or part of them. It is worth noticing that hypotheses about the factors and processes favoring the evolution of social interactions (e.g., Michener, 1974, 1985; Wcislo \& Tierney, 2009) can be dissociated from a number and timing of evolutionary events, although there are obvious advantages in jointly investigating these two fields.

Whereas the hypotheses of phylogenetic relationships among the eusocial representative taxa of allodapine bees (Apidae: Xylocopinae) and Halictinae (Halictidae) have become more stable over the years (Brady et al., 2006b; Chenoweth et al., 2007; Schwarz et al., 2007; Gibbs et al., 2012), relationships among the corbiculate bees have remained largely uncertain based on phylogenetic investigations and discussions published since the 1970's. Nevertheless, two points must be made about the systematics of corbiculate bees: (a) there is almost no doubt about the naturalness (i.e., monophyly) of the corbiculate-bee clade (e.g., Roig-Alsina \& Michener, 1993; Cardinal \& Danforth, 2011); and (b) there is little or no controversy over the natural boundaries of the four most distinctive corbiculate lineages, which are classified as the tribes Apini, Bombini, Euglossini, and Meliponini. The most recent common ancestor of corbiculate bees is estimated to have begun differentiating from the closest apid lineages at about 95-72 million years ago, during the Late Cretaceous (Cardinal et al., 2010; Cardinal \& Danforth, 2011, 2013; Martins et al., 2014). The group comprises the well-known honey bees (Apis, 11 species - Apini), bumble bees (Bombus, approximately 260 species - Bombini), orchid bees ( 5 genera, approximately 240 species - Euglossini), and stingless bees (ca. 60 genera, over 500 species - Meliponini) (Michener, 2007; Rasmussen \& Cameron, 2010; Camargo \& Pedro, 2012; Moure et al., 2012; Ascher \& Pickering, 2014). In total, corbiculate bees encompass about $5 \%$ of the described species diversity of bees known to this day, and the group is far from homogeneous in terms of social organization (particularly Meliponini and Euglossini). In contrast, the closest relatives to the corbiculate clade are the bee genera Centris and Epicharis (Cardinal \& Danforth, 2011; Martins et al., 2014), which do not display any kind of social behavior (Michener, 2007; Cardinal \& Danforth, 2011; Martins et al., 2014).

It should be a rather straightforward problem the search for the phylogenetic relationships among Apini, Bombini, Euglossini, and Meliponini, because there are only three 
possible unrooted tree topologies, based on the mathematical properties of trees (Felsenstein, 1978). For each of these unrooted networks, there are five possible rooted tree topologies, as shown in Figure 2. After comparing the empirical support each of the 15 possible cladograms received over the history, it is amazing to realize that nine of them have had at least one paper in their favor (Fig 2), although some of them are supported by many results (hypotheses H1-c, H1-d, H2-c, H2-e shown in Fig 2). The conflicting empirical support received by the various phylogenetic hypotheses of corbiculate bees has been hard to explain. The gap between hypotheses listed as $\mathrm{H} 1$ and those portrayed as $\mathrm{H} 2$ in Figure 2 is clearly related to the source of the data employed for the investigation, as molecular datasets largely support the former, and phenotypic characters (behavior and morphology) favor the latter in most cases.

Potential convergence in parts of the phenotype and artifacts related to molecular evolution have been pointed as the most likely causes for the contention (Winston $\&$ Michener, 1977; Michener, 1990; Ascher et al., 2001; Cameron \& Mardulyn, 2001; Lockhart \& Cameron, 2001; Kawakita et al., 2008), but this has not resolved the controversy about how Apini, Bombini, Euglossini, and Meliponini are related to each other. The early diversification of corbiculate bees probably happened in ways that make the ancient phylogenetic signal hardly detectable. In part, this might be explained by the extinction of various lineages of corbiculate bees since the Cretaceous (e.g., Engel, 2001a, b; Engel et al., 2009) and possibly by rapid divergence among some lineages. Choice of outgroups is a sensitive issue when working with corbiculate bee relationships (e.g., Canevazzi \& Noll, 2014), which is illustrated by the unstable placement of different lineages of Apidae near or within the corbiculate clade (Table 1 of Cardinal \& Packer, 2007). Finally, it is worth speculating the rooting is also an issue when selecting one of the alternative rooted tree topologies (right column of Fig 2) from one of the unrooted trees shown on the left of the same figure. Character data will support one of the three topologies on the left, whereas rooting (more specifically, the placement of the rootnode) will be decisive for the proposal of one rooted tree-topology as the most likely scenario for the evolutionary connections among the four corbiculate-bee lineages. The consequences of the instability of placement of the root-node are far from trivial, as for example, in $\mathrm{H} 2$ the relationships can vary from Apini as sister-group of all remaining corbiculates (Fig 2, hypothesis H2-a), Meliponini as sister to the other three apine tribes (Fig 2, hypothesis H2-b), or Meliponini as sister to Apini (Fig 2, hypotheses H2-c and H2-e). These are the possibilities 
only within the realm of the hypothesis termed ' $\mathrm{H} 2$ ' in Figure 2, but there are also three possible rootings accepted for $\mathrm{H} 1$ and two possibilities within $\mathrm{H} 3$, as can be seen in the same figure.

\section{Phylogenetic Uncertainty and Behavioral Evolution within the corbiculate clade}

How do the incongruences and the uncertainty about the phylogeny of this group of bees affect our understanding of the evolution of obligate sociality in the corbiculate clade?

If the phylogenetic hypotheses sustained by the majority of morphological and behavioral data obtained so far is correct, thus we should assume that the direct ancestor of Apini+Meliponini also presented some (or all) the traits necessary to be considered obligate eusocial too (Fig 3A, B). Alternatively, it is possible that the immediate ancestor of Apini+Meliponini+Bombini (Fig 3C) or of all four taxa (Fig 3D) already presented some or all the traits observed in obligate eusocial bees. Although the debate about single versus multiple origins of obligate eusociality has generated enthusiastic discussions over the years, it must be pondered that this is an over-simplification of a more complex case.

It is far less informative because the relation of homology being sought and investigated is not about "obligate eusociality" itself, but the suite of traits that evolve and are the requisites for this condition to emerge. If research programs are targeted on obligate eusociality, growth of knowledge about the evolution of this epiphenomenon will be likely hindered. An innovative examination of key traits most directly related to the appearance of eusociality in bees was that by Cardinal and Danforth (2011), who reconstructed states of phenotypic characters that co-occur in eusocial bees. This resulted in the reconstruction of evolutionary paths taken by the following five characters in the corbiculate clade: (a) castes/division of labor, (b) adults of two generations, (c) morphologically distinct gynes, (d) progressive feeding, and (e) swarming. A simplified version in which levels of sociality are treated as character-states was also considered, but it is way less informative than the former (Cardinal \& Danforth, 2011: Fig 1C, their "traditional" model). In all scenarios evaluated, totipotentcaste eusociality was the most likely condition estimated for the most recent common ancestor of all corbiculate bee species (Cardinal \& Danforth, 2011). Worth noting too is the extensive behavioral comparison by Noll (2002), who expanded the term "social" into no fewer than 42 characters! The continued detailed investigations of morphological and behavioral changes, as 
well as the genetic basis for eusociality to appear (e.g., Toth et al., 2007; Woodard et al., 2011) will be key for the advancement in this field.

\section{Fascinating Behavioral Variation and the Need of Comparative Research: Stingless Bees and Orchid Bees (Apidae: Meliponini and Euglossini)}

When comparing the four major lineages of corbiculate bees, the Meliponini occupy a very special position in all measures of their diversity. There are about 500 described species of stingless bees, which are distributed in various tropical regions of the planet (the distribution was wider in the past as documented by the fossil record: e.g., Engel [2011]), there is a great morphological diversity, which is reflected by the approximately 60 genera currently accepted, and great behavioral variation. The diversity of biologies of stingless bees has been documented by several researchers, many of which made important contributions to document it in comparative manners (e.g., Ihering, 1903, 1930; Kerr, 1948, 1969, 1987; Sakagami, 1982; Engels \& Imperatriz-Fonseca, 1990; Imperatriz- Fonseca \& Zucchi, 1995; Faustino et al., 2002; Tarpy \& Gilley, 2004; Tóth et al., 2004; Santos-Filho et al., 2006). Observations, experiments and predictions exist for attributes such as queen-policing, mode of queen production, worker egg-laying and cost of worker reproduction, relatedness between sisters in a colony (e.g., Engels \& Imperatriz-Fonseca, 1990; Imperatriz- Fonseca \& Zucchi, 1995; Tóth et al., 2004), making stingless bees a bionomically diverse and with an incredibly open field for comparative research. Many comparisons made in the past lacked an explicit phylogenetic framework, which have the potential of illuminating which evolutionary scenarios are more likely to explain the observed phenotypic diversity. One notable exception was the evaluation of the diversity in composition of the cuticular chemistry within the Meliponini using an explicit phylogenetic framework (Leonhardt et al., 2013).

Different authors contributed to the understanding of phylogenetic relationships within Meliponini (e.g., Schwarz, 1948; Moure, 1961; Wille, 1979; Michener, 1990; Camargo \& Pedro, 1992a, b; Costa et al., 2003; Rasmussen \& Cameron, 2010). A summary of the three most comprehensive phylogenetic hypotheses (in terms of taxon sampling) is presented in Fig 4. The great diversity of forms, questionable proposals for generic delimitation, and wide geographical distribution certainly made the task of studying all stingless bees very challenging. The comparison of three hypotheses proposed independently along the last 24 
years (Fig 4A-C) makes it clear that certain portions of the tree are very stable for example, Trigonisca, Leurotrigona and related lineages have been placed relatively distant from the remaining Neotropical genera, whereas placement of certain taxa have varied widely with the analysis. The genus Melipona, for example, is placed as sister to the majority of Neotropical meliponine genera in one hypothesis (Fig 4A; Rasmussen \& Cameron, 2010), sister of a smaller Neotropical clade (Fig 4B; Camargo \& Pedro, 1992a), or sister of all other stingless bee genera (Fig 4C; Michener, 1990).

The Neotropical genus Melipona has had an important place in the discussion of behavioral evolution of stingless bees, because of its rather unique morphological and bionomical characteristics. Some authors have argued for a classification of stingless bees in which Melipona would be clustered in its own subgroup (i.e., Meliponini s.str.), apart from the Trigona-group (Trigonini), comprising all remaining genera of Meliponini (e.g., Moure, 1961). Melipona is the sole stingless bee genus where polygynic colonies (multiple queens) are known (Bego, 1989; Alves et al., 2011); these bees are also unique for the breeding queens, males and workers in identical cells, instead of having distinctly larger royal cells in the other Meliponini (Michener, 1974; Engels \& Imperatriz- Fonseca, 1990); and the occurrence of worker parasitism related to male production (Alves et al., 2009) and alien queens infiltrate colonies whose own queen had recently died (Wenseleers et al, 2011). A robust phylogenetic framework for the Meliponini can make possible the investigation of the unique traits of Melipona, which might be autapomorphic for these bees, or be better interpreted as retention of plesiomorphic states in some cases. Behaviors related to the evolution of obligate eusociality will then be reinterpreted in historical terms.

Orchid bees (Euglossini) are another key-group for discussing the evolution of social interactions in the corbiculate clade (e.g., Soucy et al., 2003). Whereas research focused on the other corbiculate tribes (Apini, Bombini, Meliponini) mainly clarify the maintenance of elaborate social behaviors, investigation on Euglossini species are especially important because they would be able to potentially shed light on issues concerning the appearance of these social behaviors. Two genera are strictly cleptoparasitic on other euglossine bees, Aglae and Exaerete, whereas species of Euglossa, Eulaema, and Eufriesea are solitary, communal or “primitively social” (Zucchi et al., 1969; Dressler, 1982; Garófalo, 1985; Cameron, 2004; Augusto \& Garófalo, 2009). Although orchid bees are closely related to the eusocial honey 
bees, bumble bees, and stingless bees, no species of Euglossini has been reported to display eusocial behaviors comparable to what is known for the remaining corbiculate tribes. Some species in Euglossa, however, present clusters of behavioral traits that are somewhat similar to those observed in Bombini. The decision to classify them as totipotent-caste eusocial in this case would be, perhaps, arbitrary.

Unfortunately, little is known about the nesting biology and social interactions of most of the species of Euglossini. If we consider all the described species, only a small amount of $(<20 \%)$ has their nests described in some detail (Dressler, 1982; Kimsey, 1982; Garófalo, 1985, 1992; Garófalo et al., 1998; Cameron, 2004). The genus Euglossa is the best-studied group and probably the most interesting taxon for sociobiological investigation in Euglossini, with the nesting behavior described for at least six species (Garófalo, 1985, 1992; Augusto \& Garófalo, 2009; Andrade-Silva \& Nascimento, 2012). The interest on this genus is largely explained by the usual occurrence of multi-female nests (MFN) with some overlap of generations and task allocation with reproductive dominance (Augusto \& Garófalo, 2009, 2011). MFN's are commonly attained by nest reactivation by the daughters in the presence of the mother (matrifilial associations), although it can be potentially accomplished also by two or more sisters of a same generation (i.e., sororal associations). When a nest is reactivated, the mother or older sister could act as the dominant (Ramírez-Arriaga et al., 1996; Pech et al., 2008; Augusto \& Garófalo, 2009, 2011). In addition to Euglossa, there are reports of task allocation and reproductive dominance (division of labor) in the genus Eulaema (Bennett, 1965; Dodson, 1966), which is not verified in communal nests of Eufriesea (Dressler, 1982; Kimsey, 1982).

In this context, a phylogenetic framework is indispensable to fully understand the evolution of social-related traits in Euglossini (e.g., Ramírez et al., 2010), and corbiculate bees as a whole, without making erroneous assumptions on the homology of behavioral complexes. Noll (2002) remarked that many components of what are called "primitive" and "advanced" social behavior should be viewed as sets of independent characters. In this way, analyses of complex traits related to sociality should be done very carefully as in the following comparisons: (a) single-female nests (solitary) vs. multi-female nests (MFN), and matrifilial or sororal associations; (b) communal nests vs. existence of task allocation (e.g., guarding, provisioning) and evidence of reproductive dominance (i.e., oophagy, threatening/aggressive 
behavior). The knowledge about the co-occurrence of these traits and the instances that this occurs in the euglossine phylogeny is central for grasping the fine aspects of social evolution. Nonetheless, phylogenetic relationships among the five genera of Euglossini remain highly uncertain (see Cameron, 2004; Cardinal \& Danforth, 2011; Ramírez et al., 2011 for a sample of conflicting results). Better understanding of these relationships together with a much needed accumulation of behavioral data for orchid bees (particularly Euglossa) will provide the confidence needed for the resolution of enigmas regarding social evolution within Euglossini and the corbiculate clade as whole.

According to the resulting ancestral state reconstructions by Cardinal and Danforth (2011), the most likely ancestral state for corbiculate bees was facultative eusociality. Perhaps more relevant than the accuracy of the reconstruction itself is the indication of a strong signal of social interactions as pervasive in the early stages of evolution of corbiculate bees. Complex forms of communication, caste polymorphism and division of labor, and construction of complex nests could have begun its unique path of evolution in corbiculate bees in the Cretaceous, over 80 million years ago (Cardinal \& Danforth, 2011).

\section{Acknowledgements}

We dedicate this review to Professor Charles D. Michener, who has contributed to the comparative research of bees for more than 70 years. His genuine interest for the broadest and most and most general questions regarding bee morphology, behavior, classification, and evolution in general laid the basis that allow comparative research to be on the right track presently in bee research. His enthusiasm has also served as inspiration and encouragement for several generations of bee researchers who have been answering and opening avenues for the future of melittology. We would like to thank Carlos A. Garófalo, Aline Andrade-Silva, Denise A. Alves, and Túlio M. Nunes for discussions and for providing useful literature for this paper. We are grateful to Luis Roberto Faria Junior for being so kind as to carefully read and make suggestions to the manuscript, as well as for the feedback provided by three anonymous reviewers and by Denise A. Alves. This project was partly supported by grant \# 2011/09477-9, São Paulo Research Foundation (FAPESP) to E.A.B.Almeida; D.S.Porto is supported by Master's fellowships also granted by FAPESP (Nbrs. 2012/22261-8 \& 2014/10090-0). 


\section{References}

Abonyi, S. (1903). Morphologische und physiologische Beschreibung des Darmkanals der Honigbiene (Apis mellifica). Ablatt Közlem., II: 137-168.

Albrecht, M., Schmid, B., Hautier, Y. \& Muller, C.B. (2012). Diverse pollinator communities enhance plant reproductive success. Proc. Biol. Sci., 279, 4845-4852. DOI: 10.1098/rspb.2012.1621

Alexander, B. A. \& Michener, C. D. (1995). Phylogenetic studies of the families of short-tongued bees (Hymenoptera: Apoidea). Univ. Kansas Sci. Bul. 55, 377-424.

Almeida, E. A. B., Pie, M. R., Brady, S. G. \& Danforth, B. N. (2012). Biogeography and diversification of colletid bees (Hymenoptera: Colletidae): emerging patterns from the southern end of the world. J. Biogeogr. 39: 526-544. DOI: 10.1111/j.1365-2699.2011.02624.x

Alves, D. A., Menezes, C., Imperatriz-Fonseca, V. L., \& Wenseleers, T. (2010). First discovery of a rare polygyne colony in the stingless bee Melipona quadrifasciata (Apidae, Meliponini). Apidologie. 42: 211-213. DOI: 10.1051/apido/2010053

Alves, D. A., Imperatriz-Fonseca, V. L., Francoy, T. M., Santos-Filho, P. S., Nogueira-Neto, P., Billen, J. and Wenseleers, T. (2009). The queen is dead-long live the workers: intraspecific parasitism by workers in the stingless bee Melipona scutellaris. Molec. Ecol., 18: 4102-4111. DOI: 10.1111/j.1365-294X.2009.04323.X

Andrade-Silva, A. C. R. \& Nascimento, F. S. (2012). Multifemale nests and social behavior in Euglossa melanotricha (Hymenoptera, Apidae, Euglossini). J. Hymen. Res. 26: 1-16. DOI: 10.3897/JHR.26.1957

Ascher, J. S., Danforth, B. N. \& Ji, S. (2001). Phylogenetic utility of the major opsin in bees (Hymenoptera: Apoidea): a reassessment. Mol. Phylogenet. Evol. 19: 76-93. DOI: 10.1006/mpev.2001.0911

Ascher, J. S. \& Pickering, J. (2014). Discover Life bee species guide and world checklist (Hymenoptera: Apoidea: Anthophila). [Accessed: October 25, 2014] URL: http://www.discoverlife.org/mp/20q?guide=Apoidea_species

Arnhart, L. (1906). Anatomie und Physiologie der Honigbiene. Wien: M. Perles, 99 p.

Augusto, S. C. \& Garófalo, C. A. (2009). Bionomics and sociological aspects of Euglossa fimbriata (Apidae, Euglossini). Genet. Mol. Res. 8 (2): 525-538. DOI: 10.4238/vol8-2keer004

Augusto, S. C. \& Garófalo, C. A. (2011). Task allocation and interactions among females in Euglossa carolina nests (Hymenoptera, Apidae, Euglossini). Apidologie 42: 162-173. DOI: 10.1051/apido/2010040 
Bartomeus, I., Park, M. G., Gibbs, J., Danforth, B. N., Lakso, A. N. \& Winfree, R. (2013). Biodiversity ensures plant-pollinator phenological synchrony against climate change. Ecol. Letters 16: 13311338. DOI: $10.1111 /$ ele. 12170

Bennett, F. D. (1965). Notes on a nest of Eulaema terminata Smith (Hymenoptera, Apoidea) with a suggestion of the occurrence of a primitive social system. Insect. Soc. 12: 81-92.

Blair, J. E. (2009). Animals (Metazoa). In Hedges, S. B. \& Kumar, S. (Eds.), The Timetree of Life (pp. 223-230). New York: Oxford University Press.

Bordas, L. (1894). Anatomie du tube digestif des Hyménoptères. Comptes Kendus de I'Acad. des Sci., CXVIII: 1423-1425.

Bourke, A. F. G. (1999). Colony size, social complexity and reproductive conflict in social insects. J. Evol. Biol. 12: 245-257. DOI: 10.1046/j.1420-9101.1999.00028.x

Brady, S. G., Schultz, T. R., Fisher, B. L. \& Ward, P. S. (2006a). Evaluating alternative hypotheses for the early evolution and diversification of ants. Proc. Natl. Acad. Sci. USA 103: 18172-18177. DOI: $10.1073 /$ pnas.0605858103

Brady, S. G., Sipes, S., Pearson, A. \& Danforth, B. N. (2006b). Recent and simultaneous origins of eusociality in halictid bees. Proc. R. Soc. B 273, 1643-1649. DOI: 10.1098/rspb.2006.3496

Briant, T. J. (1884). On the anatomy and functions of the tongue of the honey bee (worker). Journ. Linn. Soc. London Zool.: 408-417.

Camargo, J. M. F. \& Pedro, S. R. M. (1992a). Sistemática de Meliponinae (Hymenoptera, Apidae): sobre a polaridade e significado de alguns caracteres morfológicos. In: "Anais do Encontro Brasileiro sobre Biologia de Abelhas e outros Insetos Sociais.” Naturalia, num. esp., p. 45-49.

Camargo, J. M. F. \& Pedro, S. R. M. (1992b). Systematics, phylogeny and biogeography of the Meliponinae (Hymenoptera: Apidae): a mini-review. Apidologie 23: 509-522. DOI: 10.1051/apido:19920603

Camargo, J. M. F. \& Pedro, S. R. M. (2003) Sobre as relações filogenéticas de Trichotrigona Camargo \& Moure (Hymenoptera, Apidae, Meliponini). In Melo, G. A. R. \& Alves-dos-Santos, I. (Eds.), Apoidea Neotropica: Homenagem aos 90 Anos de Jesus Santiago Moure (pp. 45-49). Criciúma: Editora UNESC.

Camargo, J. M. F. \& Pedro, S. R. M. (2013). Meliponini Lepeletier, 1836. In Moure, J. S., Urban, D. \& Melo, G. A. R. (Orgs). Catalogue of Bees (Hymenoptera, Apoidea) in the Neotropical Region online version. Available at http://www.moure.cria.org.br/catalogue. Accessed Oct/25/2014 
Camargo, J. M. F. \& Roubik, D. W. (2005). Neotropical Meliponini: Paratrigonoides mayri, new genus and species from western Colombia (Hymenoptera, Apidae, Apinae) and phylogeny of related genera. Zootaxa 1081: 33-45.

Cameron, S. A. (1991). A new tribal phylogeny of the Apidae inferred from mitochondrial DNA sequences. In Smith, D. R. (Ed.), Diversity in the Genus Apis (pp. 71-87). Oxford: Westview Press.

Cameron, S. A. (1993). Multiple origins of advanced eusociality in bees inferred from mitochondrial DNA sequences. Proc. Natl. Acad. Sci. USA 90: 8687-8691.

Cameron, S. A. (2004). Phylogeny and biology of Neotropical orchid bees (Euglossini). Annu. Rev. Entomol. 49: 377-404. DOI: 10.1146/annurev.ento.49.072103.115855

Cameron, S. A., Lozier, J. D., Strange, J. P., Koch, J. B., Cordes, N., Solter, L. F. \& Griswold, T. L. (2010). Patterns of widespread decline in North American bumble bees. Proc. Natl. Acad. Sci. USA 108(2): 662-667. DOI: 10.1073/pnas.1014743108

Cameron, S. A. \& Mardulyn, P. (2001). Multiple molecular data sets suggest independent origins of highly eusocial behavior in bees (Hymenoptera: Apinae). Syst. Biol. 50 (2): 194-214. DOI: $10.1080 / 10635150120230$

Cardinal, S. \& Danforth, B. N. (2011). The antiquity and evolutionary history of social behavior in bees. PLoS ONE 6(6): e21086. DOI: 10.1371/journal.pone.0021086

Cardinal, S. \& Danforth, B. N. (2013). Bees diversified in the age of eudicots. Proc R Soc B 280: 20122686. DOI: $10.1098 /$ rspb.2012.2686

Cardinal, S. \& Packer, L. (2007). Phylogenetic analysis of corbiculate Apinae based on morphology of the sting apparatus (Hymenoptera: Apidae). Cladistics 23: 99-118. DOI: 10.1111/j.10960031.2006.00137.x

Cardinal, S., Straka, J. \& Danforth, B. N. (2010). Comprehensive phylogeny of apid bees reveals the evolutionary origins and antiquity of cleptoparasitism. Proc. Nat. Acad. Sci. USA 107: 1620716211. DOI: 10.1073/pnas.1006299107

Carlet, G. (1884). Sur les muscles de I'abdomen de I'abeille. Comptes Rendus de I'Acad. des Sci. de Paris, XCVIII: 758-759.

Canevazzi, N. C. S. \& Noll, F. B. (2014). Cladistic analysis of self-grooming indicates a single origin of eusociality in corbiculate bees (Hymenoptera: Apidae). Cladistics: 16 pp. DOI: 10.1111/cla.12077

Chavarría, G. \& Carpenter, J. M. (1994). "Total evidence" and evolution of highly social bees. Cladistics 10: 229-258. DOI: 10.1111/j.1096-0031.1994.tb00177.x 
Chenoweth, L. B., Tierney, S. M., Smith, J. A., Cooper, S. J. B. \& Schwarz, M. P. (2007). Social complexity in bees is not sufficient to explain lack of reversions to solitary living over long time scales. BMC Evol. Biol. 7: 246(9). DOI: 10.1186/1471-2148-7-246

Costa, M. A., del Lama, M. A., Melo, G. A. R. \& Sheppard, W. S. (2003). Molecular phylogeny of the stingless bees (Apidae, Apinae, Meliponini) inferred from mitochondrial 16S rDNA sequences. Apidologie 34: 73-84. DOI: 10.1051/apido:2002051

Crespi, B. J. \& Yanega, D. (1994). The definition of eusociality. Behav. Ecol. 6: 109-115. DOI: 10.1093/beheco/6.1.109

Cronin, A.L., Molet, M., Doums, C., Monnin, T. \& Peeters, C. (2013). Recurrent evolution of dependent colony foundation across eusocial insects. Annu. Rev. Entomol. 58:37-55. DOI: 10.1146/annurev-ento-120811-153643

Crozier, R. H. \& Crozier, Y. C. (1993). The mitochondrial genome of the honeybee Apis mellifera: complete sequence and genome organization. Genetics, 133(1): 97-117.

Danforth, B. N., Cardinal, S., Praz, C., Almeida, E. A. B. \& Michez, D. (2013). The impact of molecular data on our understanding of bee phylogeny and evolution. Annu. Rev. Entomol. 58: 57-78. DOI: 10.1146/annurev-ento-120811-153633

Danforth, B.N., Fang, J. \& Sipes S. D. (2006a). Analysis of family level relationships in bees (Hymenoptera: Apiformes) using 28S and two previously unexplored nuclear genes: CAD and RNA polymerase II. Molec. Phylogen. Evol. 39: 358-372. DOI: 10.1016/j.ympev.2005.09.022

Danforth, B. N., Sipes, S.D., Fang, J. \& Brady, S. G. (2006b). The history of early bee diversification based on five genes plus morphology. Proceedings of the National Academy of Sciences of the United States of America 103: 15118-15123. DOI: 10.1073/pnas.0604033103

Debevec, A. H., Cardinal, S. \& Danforth, B. N. (2012) Identifying the sister group to the bees: a molecular phylogeny of Aculeata with an emphasis on the superfamily Apoidea. Zool. Scripta, 41: 527-535. DOI: 10.1111/j.1463-6409.2012.00549.x

Dodson, C. H. (1966). Ethology of some bees of the tribe Euglossini (Hymenoptera: Apidae). J. Kansas Entomol. Soc. 39: 607-629.

Dressler, R. L. (1982) Biology of the orchid bees. Ann. Rev. Ecol. Syst. 13: 373-394.

Edgecombe, G. D., Giribet, G., Dunn, C. W., Hejnol, A., Kristensen, R. M., Neves, R. C., Rouse, G. W., Worsaae, K. \& Sørensen, M. V. (2011). Higher-level metazoan relationships: recent progress and remaining questions. Org. Divers. Evol. 11, 151-172. DOI: 10.1007/s13127-0110044-4 
Engel, M.S. (2001a). A monograph of the Baltic amber bees and evolution of the Apoidea (Hymenoptera). Bull. Am. Mus. Nat. Hist. 259: 1-192. DOI: http://dx.doi.org/10.1206/00030090(2001)259<0001:AMOTBA>2.0.CO;2

Engel, M. S. (2001b). Monophyly and extensive extinction of advanced eusocial bees: Insights from an unexpected Eocene diversity. Proc. Natl Acad. Sci. USA 98: 1661-1664. DOI: 10.1073/pnas.98.4.1661

Engel, M. S. (2011). Systematic melittology: where to from here? Syst. Entomol. 36: 2-15. DOI: $10.1111 / \mathrm{j} .1365-3113.2010 .00544 . x$

Engel, M. S., Hinojosa-Díaz, I. A. \& Rasnitsyn, A. R. (2009). A honey bee from the Miocene of Nevada and the biogeography of Apis (Hymenoptera: Apidae: Apini). Proc. Calif. Acad. Sci. (4)60: 23-38.

Engels, W. \& Imperatriz-Fonseca, V. L. (1990). Caste development, reproductive strategies and control of fertility in honey bees and stingless bees. In Engels, W. (Ed.), Social Insects: An Evolutionary Approach to Caste and Reproduction (pp. 167-230). Heidelberg: Springer Berlin.

Faustino, C. D., Silva-Matos, E. V., Mateus, S. \& Zucchi, R. (2002). First record of emergency queen rearing in stingless bees (Hymenoptera, Apinae, Meliponini), Insectes Soc. 49: 111-113. DOI: $10.1007 / \mathrm{s} 00040-002-8287-\mathrm{x}$

Felsenstein, J. (1978). Cases in which parsimony or compatibility methods will be positively misleading. Syst. Biol. 27(4): 401-410.

Fittkau, E. J. \& Klinge, H. (1973). On biomass and trophic structure of the Central Amazonian rain forest ecosystem. Biotropica 5: 2-14.

Garibaldi, L. A., Steffan-Dewenter, I., Winfree, R., Aizen, M. A., Bommarco, R., Cunningham, S. A., Kremen, C., Carvalheiro, L. G., Harder, L. D., Afik, O., Bartomeus, I., Benjamin, F., Boreux, V., Cariveau, D., Chacoff, N. P., Dudenhöffer, J. H., Freitas, B. M., Ghazoul, J., Greenleaf, S., Hipólito, J., Holzschuh, A., Howlett, B., Isaacs, R., Javorek, S. K., Kennedy, C. M., Krewenka, K. M., Krishnan, S., Mandelik, Y., Mayfield, M. M., Motzke, I., Munyuli, T., Nault, B. A., Otieno, M., Petersen, J., Pisanty, G., Potts, S. G., Rader, R., Ricketts, T. H., Rundlöf, M., Seymour, C. L., Schüepp, C., Szentgyörgyi, H., Taki, H., Tscharntke, T., Vergara, C. H., Viana, B. F., Wanger, T. C., Westphal, C., Williams, N. \& Klein, A. M. (2013). Wild pollinators enhance fruit set of crops regardless of honey bee abundance. Science 339: 1608-1611. DOI: $10.1126 /$ science. 1230200

Garófalo, C. A. (1985). Social structure of Euglossa cordata nests (Hymenoptera: Apidae: Euglossini). Entomol. Gener. 11: 77-83. 
Garófalo, C. A. (1992). Comportamento de nidificação e estrutura de ninhos de Euglossa cordata (Hymenoptera, Apidae, Euglossini). Rev. Brasil. Biol. 52: 187-198.

Garófalo, C. A., Camillo, E., Augusto, S. C., Jesus, B. M. V. \& Serrano, J. C. (1998). Nest structure and communal nesting in Euglossa (Glossura) annectans Dressler (Hymenoptera, Apidae, Euglossini). Revta. Bras. Zool. 15: 589-596.

Gibbs, J., Brady, S. G., Sipes, S., Kanda, K. \& Danforth, B. N. (2012). Phylogeny of halictine bees supports a shared origin of eusociality for Halictus and Lasioglossum (Apoidea: Anthophila: Halictidae). Molec. Phylogen. Evol. 65: 926-939. DOI: 10.1016/j.ympev.2012.08.013

Girdwoyn, M. (1876). Anatomie et physiologie de I'abeille. Paris: Mem. de la Soc. Polonaise des Sci. Exac., VI, 39 p.

Grimaldi, D. A. \& Engel, M. S. (2005). Evolution of the Insects. New York: Cambridge University Press $755 \mathrm{p}$.

Hartfelder, K. (2000). Insect juvenile hormone: from "status quo" to high society. Brazilian Journal of Medical and Biological Research, 33(2), 157-177. Retrieved October 25, 2014, from: http://www.scielo.br/scielo.php?script=sci_arttext\&pid=S0100$879 X 2000000200003 \& \operatorname{lng}=$ en\&tlng=en. 10.1590/S0100-879X2000000200003.

Hartfelder, K. \& Engels, W. (1998). Social insect polymorphism: Hormonal regulation of plasticity in development and reproduction in the honeybee. Current Topics in Developmental Biology 40: 45-77.

Hartfelder, K., Market, G. R., Judice, C. C., Pereira, G. A. G., Santana, W. C., Dallacqua, R. \& Bitondi, M. M. G. (2006). Physiological and genetic mechanisms underlying caste development, reproduction and division of labor in stingless bees. Apidologie 37: 144-163. DOI: 10.1051/apido:2006013

Hedke, S. M., Patiny, S. \& Danforth, B. N. (2013) The bee tree of life: a supermatrix approach to apoid phylogeny and biogeography. BMC Evol. Biol., 13:138. DOI: 10.1186/1471-2148-13-138

Hines, H. M., Hunt, J. H., O'Connor, T. K., Gillespie, J. J. \& Cameron, S. A. (2007). Multigene phylogeny reveals eusociality evolved twice in vespid wasps. Proc. Natl. Acad. Sci. USA 104: 3295-3299. DOI: 10.1073/pnas.0610140104

Hölldobler, B. \& Wilson, E. O. (1990). The Ants. Cambridge: Harvard Univ. Press, 732 p.

Hommell, R. (1904). Anatomie et physiologie de I'abeille domestique. Le Microg. Prép., XII: 49-60.

Hommell, R. (1905). Anatomie et physiologie de I'abeille domestique. Le Microg. Prép., XIII: 15-25, 60-67. 
'Honeybee Genome Sequencing Consortium' (2006). Insights into social insects from the genome of the honeybee Apis mellifera. Nature 443(7114): 931-949. DOI: 10.1038/nature05260

Ihering, H. v. (1903). Biologie der stachellosen Honigbienen Brasiliens, Zool. Jahrb., Abt. Syst., Geogr. Biol. der Tiere. 19, 179-287, pl. 110-122.

Ihering H. v. (1930). Biologia das abelhas melliferas do Brasil, Bol. Agric. (São Paulo) 31, 435-506; 649-714.

Imperatriz-Fonseca, V. L. \& Zucchi R. (1995). Virgin queens in stingless bee (Apidae, Meliponinae) colonies: a review. Apidologie 26: 231-244. DOI: 10.1051/apido:19950305

Jarvis, J. U. (1981). Eusociality in a mammal: cooperative breeding in naked mole-rat colonies. Science 212: 571-573. DOI: 10.1126/science.7209555

Kamakura, M. (2011). Royalactin induces queen differentiation in honeybees. Nature 473: 478-483. DOI: $10.1038 /$ nature10093

Kawakita, A., Ascher, J. S., Sota, T., Kato, M. \& Roubik, D. W. (2008). Phylogenetic analysis of the corbiculate bee tribes based on 12 nuclear protein-coding genes (Hymenoptera: Apoidea: Apidae). Apidologie 39: 163-175. DOI: 10.1051/apido:2007046

Kerr, W. E. (1948). Estudos sôbre o gênero Melipona, An. Esc. Sup. Agricult. "Luiz de Queiroz" 5: 181-276.

Kerr, W. E. (1969). Some aspects of the evolution of social bees (Apidae). Evol. Biol. 3: 119-175.

Kerr, W. E. (1987). Sex determination in bees. XVII. Systems of caste determination in the Apinae, Meliponinae and Bombinae and their phylogenetic implications. Rev. Bras. Genet. 10: 685-694.

Kimsey, L. S. (1982). Systematics of bees of the genus Eufriesea. Univ. California Publ. Entomol. 95: i-ix, $1-125$.

Kimsey, L. S. (1984). The behavioral and structural aspects of grooming and related activities in euglossine bees (Hymenoptera: Apidae). J. Zool. 204: 541-50.

Koulianos, S., Schmid-Hempel, R., Roubik, D. W. \& Schmid-Hempel, P. (1999). Phylogenetic relationships within the corbiculate Apinae (Hymenoptera) and the evolution of eusociality. J. Evol. Biol. 12: 380-384.

Laidlaw, H. H., Jr., Pimentel-Gomes, F. \& Kerr, W. E. (1956). Estimation of the number of lethal alleles in a panmitic population of Apis mellifera L. Genetics 41: 179-188.

Lee, C. Y. (2002). Subterranean termite pests and their control in the urban environment in Malaysia. Sociobiology 40: 3-10. 
Leonhardt, S. D., Rasmussen, C. \& Schmitt, T. (2013). Genes versus environment: geography and phylogenetic relationships shape the chemical profiles of stingless bees on a global scale. Proc. R. Soc. B 280 20130680;DOI: 10.1098/rspb.2013.0680

Lindauer, M. \& Kerr, W. E. (1960). Communication between the workers of stingless bees. Bee World 41: 29-71.

Lockhart, P. G. \& Cameron, S. A. (2001). Trees for bees. Trends Ecol. Evol. 16: 84-88. DOI: http://dx.doi.org/10.1016/S0169-5347(00)02054-1

Maa, T. (1953). An inquiry into the systematics of the tribus Apidini or honeybees (Hym.). Treubia 21: $525-640$.

Macloskie, G. (1881). The endocranium and maxillary suspensorium of the bee. Amer. Nat., XV: 353362.

Mardulyn, P. \& Cameron, S. A. (1999). The major opsin in bees (Insecta: Hymenoptera): a promising nuclear gene for higher level phylogenetics. Mol. Phylogenet. Evol. 12: 168-176. DOI: 10.1006/mpev.1998.0606

Martins, A. C., Melo, G. A. R. \& Renner, S. S. (2014). The corbiculate bees arose from New World oil-collecting bees: Implications for the origin of pollen baskets. Molec. Phylogen. Evol. 80, 8894. DOI: 10.1016/j.ympev.2014.07.003

Melo, G. A. R. (1999). Phylogenetic relationships and classification of the major lineages of Apoidea (Hymenoptera) with emphasis on the crabronid wasps. Scient Papers 14: 1-55.

Michener, C. D. (1944). Comparative external morphology, phylogeny, and a classification of the bees (Hymenoptera). Bull. Am. Mus. Nat. Hist. 82: 151-326.

Michener, C. D. (1974). The Social Behavior of the Bees: A Comparative Study. Cambridge: Belknap, $404 \mathrm{p}$.

Michener, C. D. (1985). From solitary to eusocial: Need there be a series of intervening species? Fortschr. Zool. 31: 293-306.

Michener, C. D. (1990). Classification of Apidae (Hymenoptera). Univ. Kansas Sci. Bull. 54: 75-164.

Michener, C. D. (2007). The Bees of the World ( $2^{\text {nd }}$ Ed). Baltimore: John Hopkins University Press, $953 \mathrm{p}$.

Moreau, C. S., Bell, C. D., Vila, R., Archibald, S. B. \& Pierce N. E. (2006). Phylogeny of the ants: Diversification in the age of angiosperms. Science 312: 101-104. DOI: $10.1126 /$ science. 1124891

Moure, J. S. (1961). A preliminary supra-specific classification of the Old World Meliponinae bees (Hymenoptera, Apoidea). Studia Entomol. 4, 181-242. 
Moure, J. S., Melo, G. A. R. \& Faria, L. R. R., Jr. (2012). Euglossini Latreille, 1802. In Moure, J. S., Urban, D. \& Melo, G. A. R. (Orgs). Catalogue of Bees (Hymenoptera, Apoidea) in the Neotropical Region - online version. Available at http://www.moure.cria.org.br/catalogue. Accessed Oct $/ 25 / 2014$

Nixon, K. C. \& Carpenter, J. M. (2012). On homology. Cladistics 28: 160-169. DOI: 10.1111/j.10960031.2011.00371.x

Noll, F. B. (2002). Behavioral phylogeny of corbiculate Apidae (Hymenoptera; Apinae), with special reference to social behavior. Cladistics 18: 137-153. DOI: 10.1006/clad.2001.0191

Oldroyd, B. P. (2007). What's killing american honey bees? PLoS Biol 5(6): e168. DOI:10.1371/journal.pbio.0050168

Page, R. E., Jr. (2013). The Spirit of the Hive: The Mechanisms of Social Evolution. Cambridge: Harvard University Press, 240 p.

Patel, A., Fondrk, M. K., Kaftanoglu, O., Emore, C., Hunt, G., Frederick, K. \& Amdam, G. V. (2007). The Making of a queen: TOR pathway is a key player in diphenic caste development. PLoS ONE 2(6): e509. doi:10.1371/journal.pone.0000509

Payne, A. (2013). Resolving the relationships of apid bees (Hymenoptera: Apidae) through a direct optimization sensitivity analysis of molecular, morphological, and behavioral characters. Cladistics: 1-15. DOI: $10.1111 /$ cla.12022

Pech, M. E. C., May-Itzá, W. de J., Medina, L. A. M. \& Quezada-Euán, J. J. G. (2008). Sociality in Euglossa (Euglossa) viridissima Friese (Hymenoptera, Apidae, Euglossini). Insect. Soc. 55: 428433. DOI: $10.1007 / \mathrm{s} 00040-008-1023-4$

Peixoto, E. B. M. I. \& Serrão, J. E. (2001). A comparative study of the cardia and cardiac valves in the corbiculate bees (Hymenoptera: Apinae). Sociobiology 37: 707-721.

Pereira-Martins, S. R., Kerr, W. E. (1991). Biologia de Eulaema nigrita. 1. Construcão de células, oviposicão e desenvolvimento. Pap. Avuls. Zool. 37: 227-235.

Plant, J. D. \& Paulus, H. F. (1987). Comparative morphology of the postmentum of bees (Hymenoptera: Apoidea) with special remarks on the evolution of the lorum. Zeitschrift fur Zoologische Systematik und Evolutionsforchung 25: 81-103.

Potts, S. G., Biesmeijer, J. C., Kremen, C., Neumann, P. \& Schweiger, O. (2010). Global pollinator declines: trends, impacts and drivers. Trends Ecol. Evol. 25(6): 345-353. DOI: 10.1016/j.tree.2010.01.007

Prentice, M. (1991). Morphological analysis of the tribes of Apidae. In Smith, D. R. (Ed.), Diversity in the Genus Apis (pp. 51-69). Oxford: Westview Press. 
Ramírez, S. R., Roubik, D. W., Skov, C. \& Pierce, N. E. (2010). Phylogeny, diversification patterns and historical biogeography of euglossine orchid bees (Hymenoptera: Apidae). Biol. J. Linn. Soc. 100: 552-572. DOI: 10.1111/j.1095-8312.2010.01440.x

Ramírez-Arriaga, E., Cuadriello-Aguilar, J. I. \& Hernández, E. M. (1996). Nest structure and parasite of Euglossa atroveneta Dressler (Apidae: Bombinae: Euglossini) at Unión Juárez, Chiapas, México. J. Kans. Entomol. Soc.69: 144-152

Rasmussen, C. \& Cameron, S. A. (2010). Global stingless bee phylogeny supports ancient divergence, vicariance, and long distance dispersal. Biol. J. Linn. Soc. 99: 206-232. DOI: 10.1111/j.10958312.2009.01341.x

Reeve, H. K. \& Keller, L. (1999). Levels of Selection in Evolution. Princeton: Princeton University Press, 318p.

Roig-Alsina, A. \& Michener, C. D. (1993). Studies of the phylogeny and classification of long-tongued bees (Hymenoptera: Apoidea). Univ. Kansas Sci. Bull. 55: 123-160.

Roubik, D. W., Segura, J. A. L. \& Camargo, J. M. F. (1997). New stingless bee genus endemic to Central American cloudforests: phylogenetic and biogeographical implications (Hymenoptera: Apidae: Meliponini). Syst. Entomol. 22: 67-80.

Sakagami S. F. (1982). Stingless bees. In Hermann H.R. (Ed.), Social insects (pp. 361-423). New York: Academic Press.

Sakagami, S. F. \& Maeta, Y. (1984). Multifemale nests and rudimentary castes in the normally solitary bee Ceratina japonica (Hymenoptera: Xylocopidae). J. Kans. Entomol. Soc. 57: 639-656.

Santos-Filho, P. de S., Alves, D. de A., Eterovic, A., Imperatriz-Fonseca, V. L. \& Kleinert, A. de M. P. (2006). Numerical investment in sex and caste by stingless bees (Apidae: Meliponini): a comparative analysis. Apidologie 37: 207-221.

Schmid, A. \& Kleine, G. (1861). Umrisse zur anatomie und physiologie der bienen. Die Bienenzeitung, I: 498-525.

Schultz, T. R., Engel, M. S. \& Prentice, M. (1999). Resolving conflict between morphological and molecular evidence for the origin of eusociality in the "corbiculate" bees (Hymenoptera: Apidae): a hypothesis-testing approach. Uni. Kans. Nat. Hist. Mus. Spec. Publ. 24: 125-138.

Schwarz, H. F. (1948) Stingless bees (Meliponidae) of the Western Hemisphere. Bull. Am. Mus. Nat. Hist. 90: 546+xviii.

Schwarz, M. P., Richards, M. H. \& Danforth, B. N. (2007). Changing paradigms in insect social evolution: insights from halictine and allodapine bees. Annu. Rev. Entomol. 52: 127-150. DOI: 10.1146/annurev.ento.51.110104.150950 
Seeley, T. D. (1995). The Wisdom of the Hive: the social physiology of honey bee colonies. Cambridge: Harvard University Press, 318p.

Seeley, T. D. (2010). Honeybee Democracy. Princeton: Princeton University Press, 280 p.

Serrão, J. E. (2001). A comparative study of the proventricular structure in corbiculate apinae (Hymenoptera: Apidae). Micron 32: 379-385. DOI: 10.1016/S0968-4328(00)00014-7

Sheppard, W. S. \& McPheron, B. A. (1991). Ribosomal DNA diversity in Apidae. In Smith, D. R. (Ed.), Diversity in the genus Apis (pp. 89-102). Oxford: Westview Press.

Snodgrass, R. E. (1910). The Anatomy of the Honey Bee. Washington: Government Printing Office, $236 \mathrm{p}$.

Snodgrass, R. E. (1935). Principles of insect morphology. New York: McGraw-Hill Book Co., ix + 667 p.

Snodgrass, R. E. (1942). The skeleto-muscular mechanisms of the honey bee. Smithson. misc. Coll. 103(2): 1-120.

Snodgrass, R. E. (1956). Anatomy of the Honey Bee. Ithaca: Cornell University Press., i-xiv + 334 p.

Soucy, S. L., Giray, T. \& Roubik, D. W. (2003). Solitary and group nesting in the orchid bee Euglossa hyachintina (Hymenoptera, Apidae). Insect. Soc. 50: 248-255. DOI: 10.1007/s00040-003-06708

Sullivan, J. P., Jassim, O., Fahrbach, S. E. \& Robinson, G. E. (2000). Juvenile hormone paces behavioral development in the adult worker honey bee. Hormones and behavior, 37(1): 1-14. DOI: 10.1006/hbeh.1999.1552

Tarpy, D. R. \& Gilley. D.C. (2004). Group decision making during queen production in colonies of highly eusocial bees. Apidologie 35: 207-216. DOI: 10.1051/apido:2004008

Toth, A. L., Varala, K., Newman, T. C., Miguez, F. E., Hutchison, S. K., Willoughby, D. A., Simons, J. F., Egholm, M., Hunt, J. H., Hudson, M. E. \& Robinson, G. E. (2007). Wasp gene expression supports an evolutionary link between maternal behavior and eusociality. Science 318: 441. DOI: $10.1126 /$ science. 1146647

Tóth, E., Queller, D. C., Dollin, A. \& Strassmann, J. E. (2004). Conflict over male parentage in stingless bees. Insect. Soc. 51: 1-11. DOI: 10.1007/s00040-003-0707-z

Trivers, R. L. \& Hare H. (1976). Haplodiploidy and the evolution of the social insects. Science. 191: 249-263. DOI: 10.1126/science. 1108197

Von Frisch, K. (1946). Die Tänze der Bienen. Österreichische Zoolog. Zeitsch. 1: 1-48 
Ware, J. L., Grimaldi, D. A. \& Engel, M. S. (2010). The effects of fossil placement and calibration on divergence times and rates: An example from the termites (Insecta: Isoptera). Arthropod Struct. Dev. 39: 204-219. DOI: 10.1016/j.asd.2009.11.003

Wcislo, W. T. \& Tierney, S. M. (2009). Evolution of communal behavior in bees and wasps: An alternative to eusociality. In J. Gadau \& J. Fewell (Eds.), Organizations of insect societies: from genome to sociocomplexity (pp. 148-169). Cambridge: Harvard University Press.

Weaver, N. (1955). Rearing of honeybee larvae on royal jelly in the laboratory. Science 121: 509-510. DOI: $10.1126 /$ science.121.3145.509

Wille, A. (1979). Phylogeny and relationships among the genera and subgenera of stingless bees (Meliponinae) of the world. Rev. Biol. Trop. 27: 241-277.

Wilson. E. O. (1971). The Insect Societies. Cambridge: The Belknap Press of Harvard University Press, $\mathrm{x}+548 \mathrm{p}$.

Wilson, E. O. \& Hölldobler, B. (2005). Eusociality: Origin and consequences. Proc. Natl. Acad. Sci. USA 102(38):13367-13371. DOI: 10.1073/pnas.0505858102

Winston, M. L. (1987). The Biology of the Honey Bee. Cambridge: Harvard University Press, 294 p.

Winston, M. L. \& Michener, C. D. (1977). Dual origin of highly social behavior among bees. Proc. Natl. Acad. Sci. USA 74: 1135-1137.

Woodard, S. H., Fischman, B. J., Venkat, A., Hudson, M. E., Varala, K., Cameron, S. A., Clark, A. G. \& Robinson, G. E. (2014). Genes involved in convergent evolution of eusociality in bees. Proc. Natl Acad. Sci. USA 108: 7472-7477. DOI: 10.1073/pnas.1103457108

Zucchi, R., Sakagami, S. F. \& Camargo, J. M. F. (1969). Biological observations on a neotropical parasocial bee, Eulaema nigrita, with a review on the biology of Euglossinae (Hymenoptera: Apidae). A comparative study. Jour. Fac. Sci. Hokkaido Univ., Zool. 17: 271-380. 


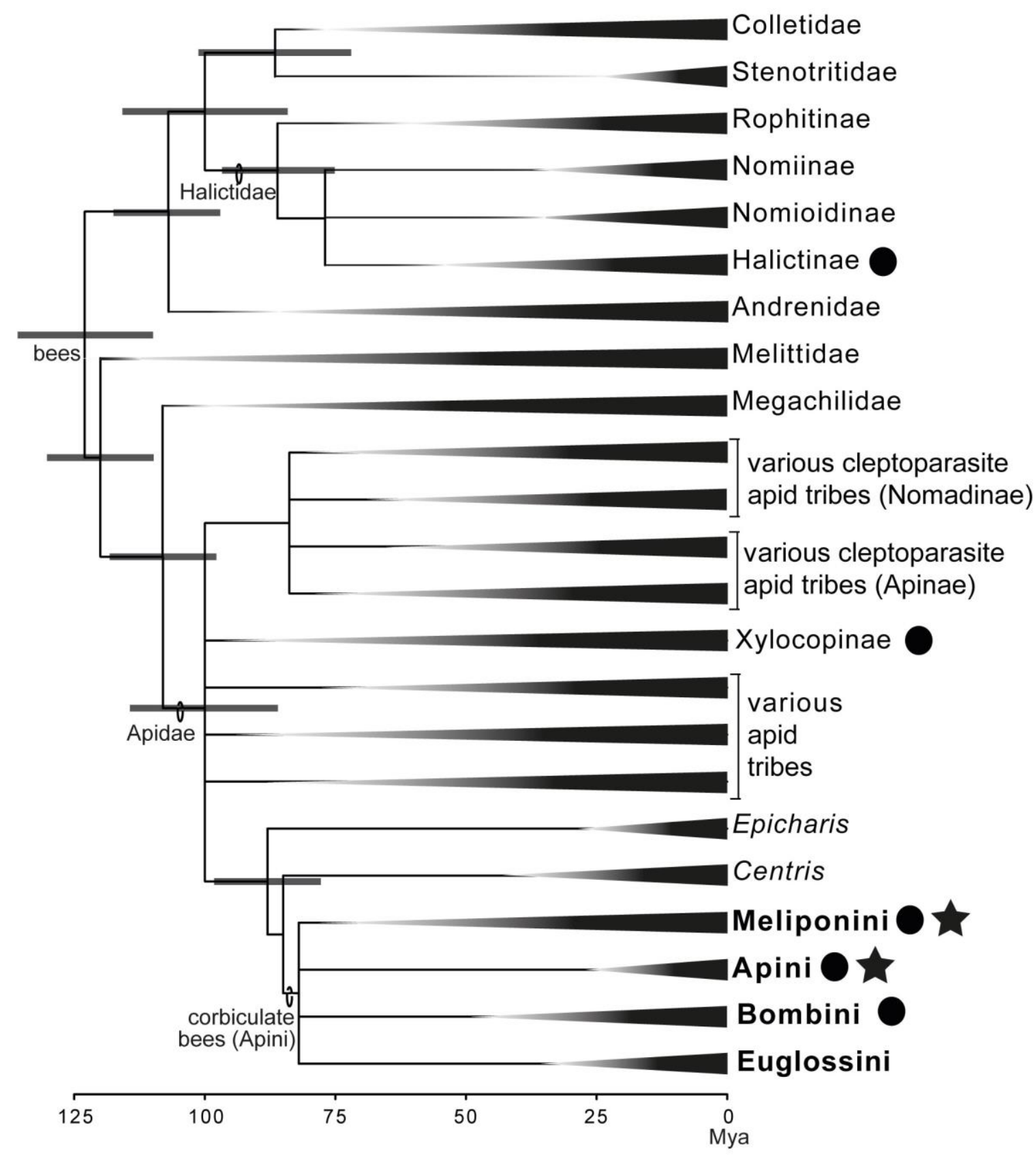

Figure 1. Phylogenetic relationships within bees (Hymenoptera: Apoidea: Anthophila). The overall relationships depicted are those summarized by Danforth et al. (2013). Bee lineages are represented at family-level resolution for taxa not comprising eusocial representatives, or in more detail in cases where eusocial species are present (indicated by solid circles, stars indicate the bee taxa considered obligate eusocial) — see Michener (2007) and Schwarz et al. (2007) for details. Unresolved nodes (i.e., polytomies) and gray shaded branches indicate regions of the tree of bees upon which uncertainty and conflicts have been most noticeable in studies published during the last decade. Branch lengths are proportional to the evolutionary time, and a ruler scaled in million years before the present is given beneath the tree (divergence ages and the estimates for clade ages were taken primarily from Cardinal \& Danforth [2013], and complemented by estimates by Brady et al. [2006b], Cardinal et al. [2010], Almeida et al. [2012], Gibbs et al. [2012], and Martins et al. [2014]). 
Figure 2. (next page) The corbiculate bee clade comprises four monophyletic groups: Apini, Bombini, Euglossini, and Meliponini. There are only three possible unrooted tree topologies that enable the representation of alternative scenarios for these four taxa, which are illustrated by the diagrams on the left $(\mathrm{H} 1-\mathrm{H} 3)$. On the right column, the five possible rooted topologies are given to the corresponding three diagrams. Published hypotheses supporting each of the rooted tree topologies are given inside circles and can be tracked by the following reference numbering system: <01> Cameron (1991); <02> Koulianos et al. (1999); <03> Schultz et al. (1999); <04> Cameron \& Mardulyn (2001); <05> Kawakita et al. (2008); <06> Cardinal et al. (2010); <07> Cardinal \& Danforth (2011); <08> Cardinal \& Danforth (2013); <09> Sheppard \& McPheron (1991); <10> Cameron (1993); <11> Koulianos et al. (1999); $<12>$ Mardulyn \& Cameron (1999); <13> Kerr (1987); <14> Winston \& Michener (1977); <15> Kimsey (1984); <16 >Sakagami \& Maeta (1984); <17> Michener (1974); <18> Michener (1990); <19> Schultz et al. (1999); <20> Serrão (2001); <21> Michener (1944); <22> Maa (1953); <23> Michener (1990); $<24>$ Prentice (1991); <25> Roig-Alsina \& Michener (1993); <26> Chavarría \& Carpenter (1994); <27> Schultz et al. (1999); <28> Ascher et al. (2001); <29> Engel (2001a, b); <30> Noll (2002); <31> Cardinal \& Packer (2007); <32> Payne (2013); <33> Canevazzi \& Noll (2014); <34> Plant \& Paulus (1987); <35> Peixoto \& Serrão (2001); <36> Pereira-Martins \& Kerr (1991). Placement of outgroup taxa (i.e., non-corbiculate bees) was ignored when accounting for the support of published hypotheses in relation to the 15 possible rooted cladograms. All unrooted tree topologies were favored by at least one published study as noticeable by references listed in the figure; and nine out of the fifteen possible rooted tree topologies were favored by at least one study (black trees on the right of the figure). Three points are worth highlighting when evaluating the support given by the various types of data and/or authors about these alternative phylogenetic scenarios: (1) there is a clear prevalence of molecular data supporting hypotheses shown in $\mathrm{H} 1$; (2) there is a clear prevalence of phenotypic genotypic data supporting hypotheses shown in $\mathrm{H} 2$; (3) scenarios shown in $\mathrm{H} 3$ have received lower support by empirical data than $\mathrm{H} 1-2$. 

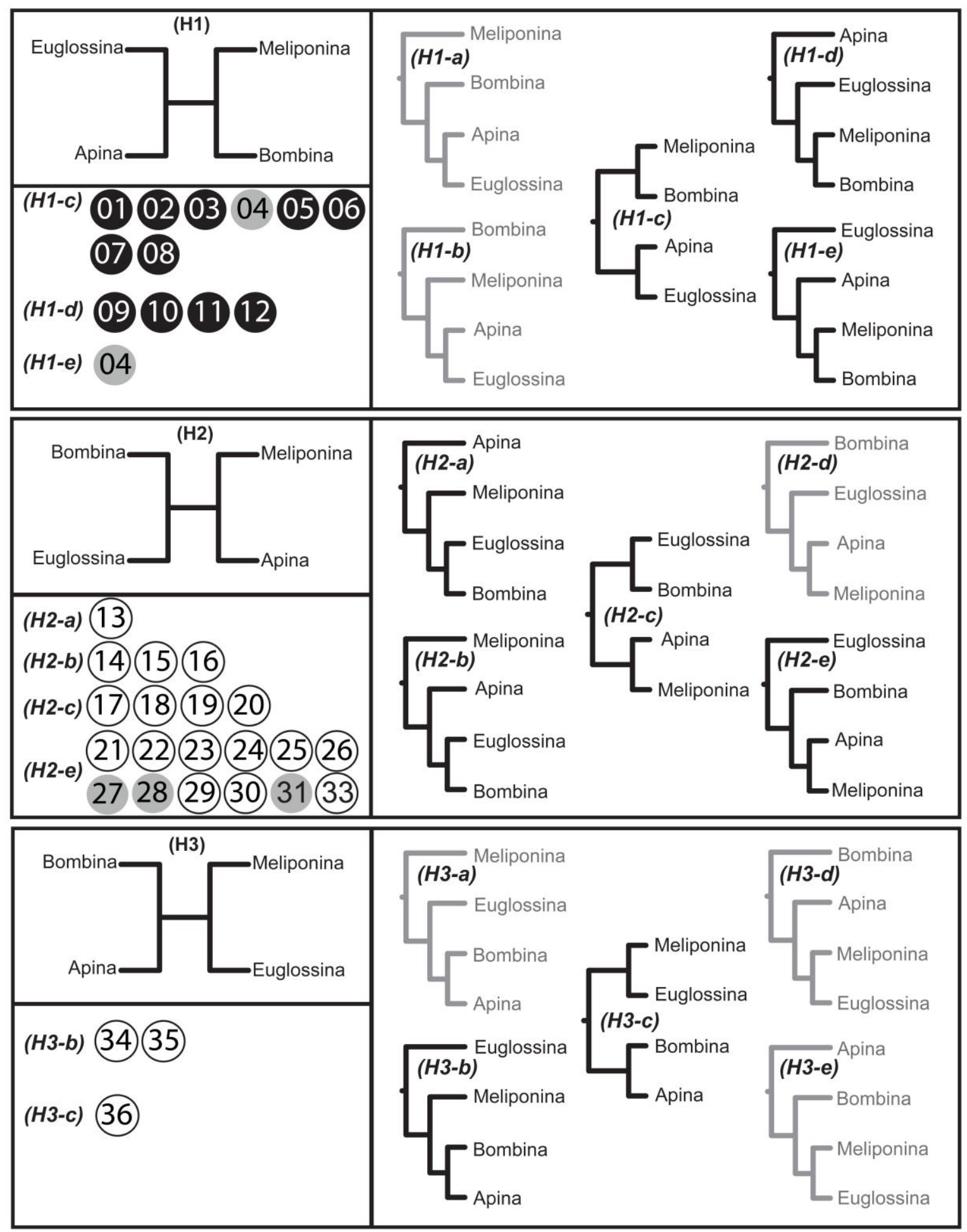
00 -molecular evidence (0) - phenotypic evidence 00 «phenotypic and molecular Numbers inside circles refer to the studies reporting one given phylogenetic hypothesis (see figure legend for the citations) 

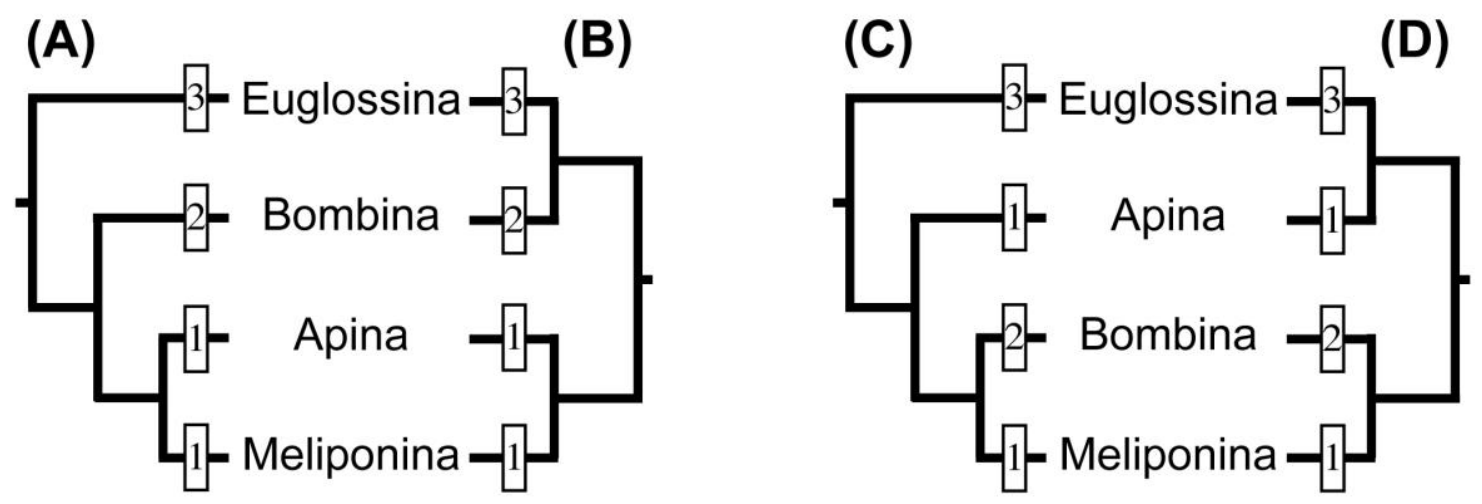

Figure 3. Schematic representation of four commonly proposed hypotheses of phylogenetic relationships among the four tribes of corbiculate bees (Apidae). Hypotheses $A$ and $B$ are favored by the several datasets comprising phenotipical characters (Fig. 2: hypothesis $\mathrm{H} 2-\mathrm{e}$ and $\mathrm{H} 2-\mathrm{c}$ ), whereas Hypotheses $C$ and $D$ are recurrent results when these relationships are inferred using molecular datasets (Fig. 2: hypothesis $\mathrm{H} 1-\mathrm{e}$ and $\mathrm{H} 1-\mathrm{c}$ ). Numbers at each of the terminal branches represent conditions of sociality: 1: fixed-caste eusociality; 2: totipotent-caste eusociality; 3: social behaviors present, but not eusociality. Tree topologies A-C correspond to hypotheses $\mathrm{H} 2-\mathrm{e}, \mathrm{H} 2-\mathrm{c}, \mathrm{H} 1-\mathrm{e}, \mathrm{H} 1-\mathrm{C}$ (Fig. 2), respectively. 
Figure 4. (next page) Summary of the phylogenetic relationships among the genera of stingless bees (Apidae: Meliponini) as proposed by (A) Rasmussen and Cameron (2010) - molecular dataset; (B) Camargo and Pedro (1992a), accompanied by a more detailed account of relationships among Neotropical genera later studied by Camargo and Pedro (2003) on the left-both morphological datasets; and (C) Michener (1990)-morphological dataset- showing two alternative scenarios for the inner relationships of one of the clades. The genus-level classification was standardized to match Rasmussen \& Cameron's classification and thus make the three hypotheses readily comparable. When one of the three analyses did not include a given genus, a putative position was defined for it and dashed lines represented the placement of this genus. For example, the following five genera were not originally included in the analysis by Rasmussen and Cameron (2010): Camargoia, Cleptotrigona, Meliwillea, Papuatrigona, and Paratrigonoides; putative placement of these in this summary tree was based on hypotheses and comments by various authors (Michener, 1990; Roubik et al., 1997; Camargo \& Pedro, 2004; Camargo \& Roubik, 2005), as previously indicated by Rasmussen and Cameron (2010). 

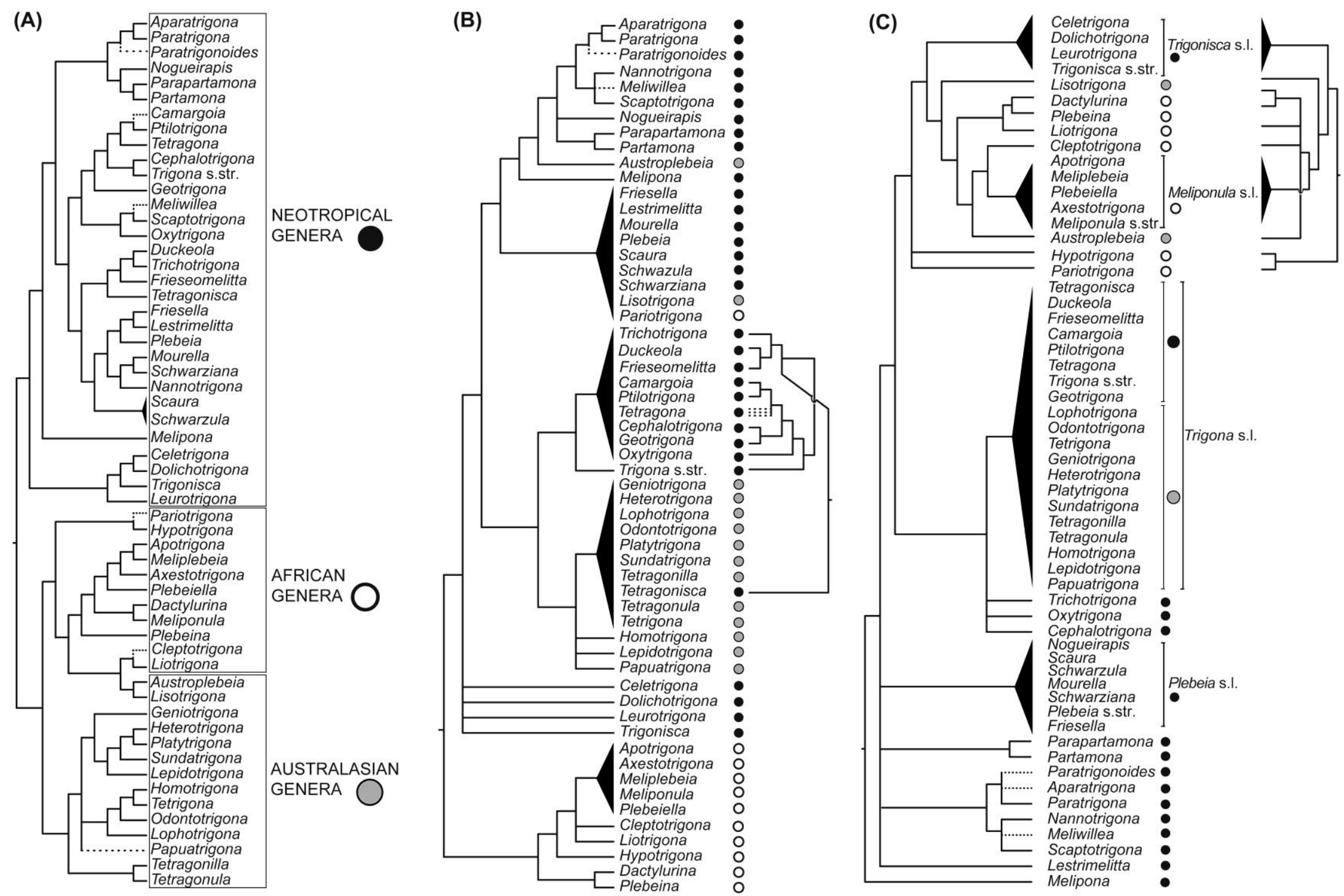